\title{
SEARCH FOR THE STANDARD MODEL HIGGS BOSON IN $e \tau$ FINAL STATES
}

\author{
by \\ IAN JAMES HOWLEY
}

\author{
Presented to the Faculty of the Graduate School of \\ The University of Texas at Arlington in Partial Fulfillment \\ of the Requirements \\ for the Degree of
}

DOCTOR OF PHILOSOPHY

THE UNIVERSITY OF TEXAS AT ARLINGTON

May 2013 
To my family. Without your support and guidance none of this is possible. You taught me that it takes passion and dedication to achieve greatness.

You mean everything to me.

Thank you. 


\section{FOREWARD}

On October 12, 1994 I wrote my daily $3^{\text {rd }}$ grade journal entry:

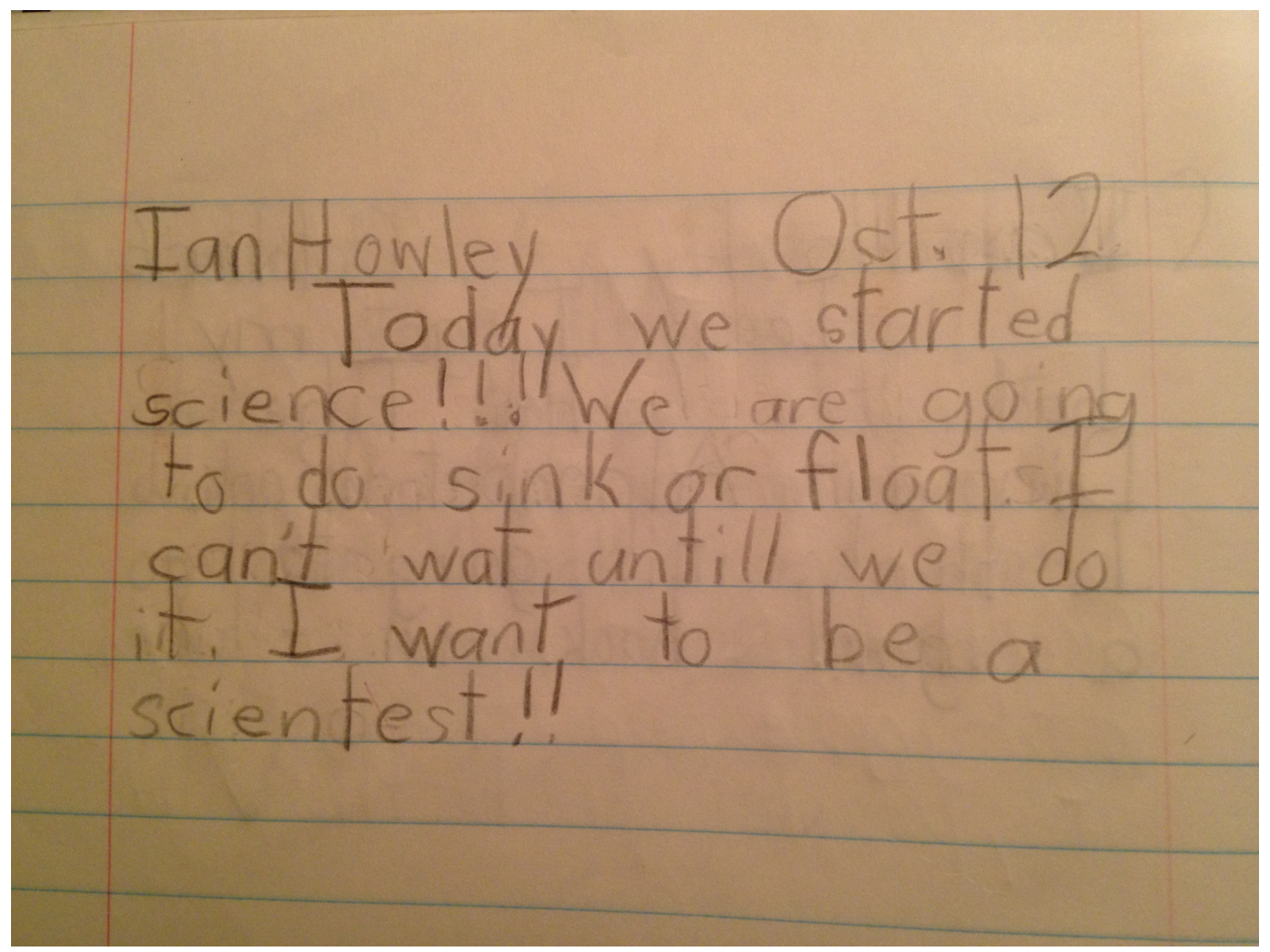

The spelling may not be the best, but clearly I was excited about science. Today I take an important step in achieving my goal of becoming a scientist. I hope that I am doing nine-year-old Ian proud. 


\section{ACKNOWLEDGEMENTS}

First and foremost I must thank my parents. You sacrificed so much in order to provide for me and my siblings. We owe you a debt of gratitude that we can only hope to pay back with love and kindness.

Thank you to my siblings: Debbie, Andrew and Rebecca. A little bro could never hope for three better examples. Your driven pursuits inspired me to follow my passions and strive for excellence. Thank you to my nieces and nephews: Abby, Sam, Cate, Declan and Dominic. Your youth and enthusiasm made it difficult to be away for five years. I can only hope that my example can inspire you to pursue your dreams.

Thank you to my best friend Nathan. We made it buddy, a long long way from Hans von Bayer's Physics 101.

Thank you to my advisor Andrew Brandt. You called me before I even arrived on campus to give me a chance, I hope that I held up my end of the bargain. We may have had to skip meals at test beam, but I will always be grateful for the experience and expertise you have graciously given to me.

Many thanks to the $\tau \tau$ team: Paul, Subhendu, Wanyu and Katy for adopting me into the Stony Brook group and guiding me through this research. Also thank you to the entire the $\mathrm{D} \varnothing$ Collaboration particularly Abid, Fabrice, Louise, Aurelio, Wade, Joe, Ken, Lidija and Boris.

Thank you to my graduate classmates: John, Miguel, Billy, Heather, Shree, Shane, Harsha, Smita, Kim, Prasad, Kapil, Ximena, Ajani, Karthik, Kassi, Mark and Arnab. And to my undergraduate crew: Jeb, Kevin and Sophie. There were 
numerous times that I felt utterly lost and one of you was kind enough to help me through to some understanding. I hope I have, or can return the favor someday.

Finally I would like to thank God, creator of the Universe. Galileo put it best when he said, "I do not feel obliged to believe that the same God who has endowed us with sense, reason and intellect has intended us to forgo their use." I hope that through striving to understand this world, it can lead me to a better understanding of the Truth.

March 29, 2013 


\begin{abstract}

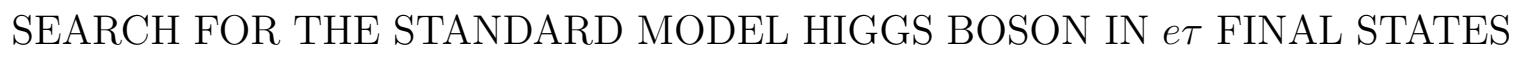
IAN JAMES HOWLEY, Ph.D.
\end{abstract}

The University of Texas at Arlington, 2013

Supervising Professor: Andrew Brandt

Presented in this dissertation is a search for the standard model (SM) Higgs boson using the DØ detector at Fermilab in Batavia, IL. The SM is a fantastically accurate theory describing the fundamental interactions and particles of the Universe. The only undiscovered particle in the SM is the Higgs boson, which is hypothesized to be responsible for electroweak symmetry breaking and giving mass to all other particles. Considered in this search is the process $H+X \rightarrow e \tau_{h} j j$, where $e$ is an electron, $\tau_{h}$ is the hadronic decay of a tau, and $j$ is a jet, using $p \bar{p}$ collisions at center of mass energy $\sqrt{s}=1.96 \mathrm{TeV}$. This search includes three production modes: associated production, gluon fusion and vector boson fusion. It also utilizes two decay channels: $H \rightarrow \tau \tau$ and $H \rightarrow W W$. A new technique, dubbed the Global Boosted Decision Tree, is introduced which offers a means of providing continuity to a multivariate search as a function of a particular parameter, in this case, the mass of the Higgs boson. The observed (expected) limit on the ratio of cross section times branching fraction to the SM at $95 \%$ confidence level is $14.6(16.0)$ at $m_{H}=125 \mathrm{GeV}$. This result is combined with the related channel $H+X \rightarrow \mu \tau_{h} j j$ and produced an observed (expected) limit of $9.0(11.3)$ at $m_{H}=125 \mathrm{GeV}$. 


\section{TABLE OF CONTENTS}

ACKNOWLEDGEMENTS ............................. iv

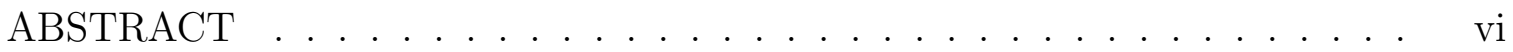

LIST OF ILLUSTRATIONS . . . . . . . . . . . . . . . . . . . . . . xi

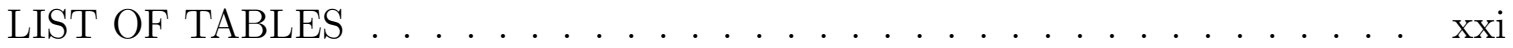

Chapter Page

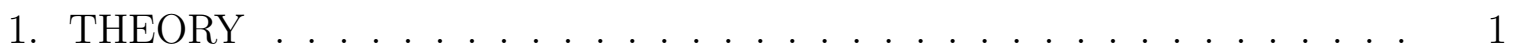

1.1 Introduction . . . . . . . . . . . . . . . . . . 1

1.2 The Tau Lepton . . . . . . . . . . . . . . . . . . . . . . . . . 3

1.3 The Standard Model . . . . . . . . . . . . . . . . . . . . . 4

1.3.1 Symmetry Breaking - The Higgs Mechanism . . . . . . . . . . 6

1.4 Higgs Boson Current Measurements . . . . . . . . . . . . . . . . . . . 10

1.4 .1 Direct Searches . . . . . . . . . . . . . . . . 11

1.4 .2 Indirect Searches . . . . . . . . . . . . . . . . . . 11

1.4.3 Theoretical Higgs Limits . . . . . . . . . . . . . . . . . . . . . 12

1.5 Higgs at the Tevatron . . . . . . . . . . . . . . . 13

1.5.1 Why $H \rightarrow \tau \tau ? \ldots \ldots . \ldots \ldots$

1.5.2 $H \rightarrow \tau \tau$ Production and Decay modes . . . . . . . . . . . 17

1.6 Overview . . . . . . . . . . . . . . . . . . . . . . 20

2. EXPERIMENTAL SETUP . . . . . . . . . . . . . . . . 21

2.1 The FermiLab Accelerator Complex . . . . . . . . . . . . . . . 21

2.1.1 Accelerator Operations . . . . . . . . . . . . . . 23

2.2 The D $\varnothing$ Detector . . . . . . . . . . . . . . . . . . 24 
2.2 .1 Tracking . . . . . . . . . . . . . . . 26

2.2 .2 Calorimeter . . . . . . . . . . . . . . . . . . 31

2.2 .3 Muon System . . . . . . . . . . . . . . . . . . . . . . 33

2.2 .4 Luminosity Monitors . . . . . . . . . . . . . . . . . . 36

2.2 .5 Trigger and Data Acquisition . . . . . . . . . . . 37

2.3 Monte Carlo Simulation . . . . . . . . . . . . . . . . . . 38

3. OBJECT IDENTIFICATION . . . . . . . . . . . . . . . . . 41

3.1 Electron Identification $\ldots \ldots \ldots \ldots$. . . . . . . . . . . 41

3.2 Tau Identification . . . . . . . . . . . . . . . . . . . . . 43

3.2.1 Tau ID Certification with the New $\mathrm{NN}_{\tau} \ldots \ldots \ldots \ldots$

3.3 Jet Identification $\ldots \ldots \ldots \ldots$

3.3 .1 Jet Algorithms . . . . . . . . . . . . . . . . . . . . 48

3.3.2 Jet Energy Scale . . . . . . . . . . . . . . . . . . . . . . 49

3.4 Missing Energy Identification $\ldots \ldots \ldots \ldots$

3.5 Muon Identification . . . . . . . . . . . . . . . . . . . . . 51

4. EVENT SELECTION . . . . . . . . . . . . . . . . . . 52

4.1 Data . . . . . . . . . . . . . . . . . . 52

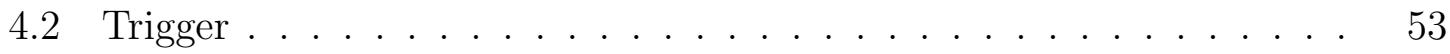

4.3 Backgrounds . . . . . . . . . . . . . . . . . 55

$4.3 .1 \quad Z+$ jets $\ldots \ldots \ldots \ldots \ldots \ldots \ldots \ldots \ldots \ldots$

$4.3 .2 \quad W+$ jets $\ldots \ldots \ldots \ldots \ldots \ldots \ldots \ldots \ldots \ldots \ldots$

$4.3 .3 t \bar{t} /$ di-boson $\ldots \ldots \ldots \ldots \ldots \ldots \ldots \ldots \ldots$

4.3 .4 Multi-jet . . . . . . . . . . . . . . . 57

4.4 Object Selection . . . . . . . . . . . . . . . . . . 58

4.4 .1 Electron Selection . . . . . . . . . . . . . . . 59

4.4 .2 Tau Selection . . . . . . . . . . . . . . . . . 60 viii 
4.4 .3 Missing Mass Calculator . . . . . . . . . . . . . . . . 61

4.4 .4 Jets Selection . . . . . . . . . . . . . . . . 63

4.4.5 Missing Energy Selection . . . . . . . . . . . . . . . 63

4.5 Multi-jet Background Estimation . . . . . . . . . . . . . 64

4.6 Event Yields . . . . . . . . . . . . . . . . . . . . . . . . . . . . 67

4.6.1 New $\mathrm{NN}_{\tau}$ and Electron MVA . . . . . . . . . . 69

5. MULTIVARIATE ANALYSIS . . . . . . . . . . . . . . . . 72

5.1 Input Variables $\ldots \ldots \ldots \ldots \ldots \ldots \ldots \ldots \ldots$

5.2 MVA Strategy . . . . . . . . . . . . . . . . . 86

$5.3 \mathrm{~T}$ and $\mathrm{W}$ Subsamples . . . . . . . . . . . . . . . 87

5.4 Initial BDT Studies . . . . . . . . . . . . . . . 88

5.5 Global BDT Method . . . . . . . . . . . . . . . . . . 90

$5.6 \quad$ BDT Outputs . . . . . . . . . . . . . . . . . . . . . . . . 93

6. LIMIT CALCULATIONS . . . . . . . . . . . . . . . . . . 101

6.1 Systematic Uncertainties . . . . . . . . . . . . . . . . . . 101

6.1.1 Normalization Systematics . . . . . . . . . . . . . . . . 101

6.1.2 Shape Systematics . . . . . . . . . . . . . . . . . . 103

6.1.3 Incorporating Systematic Uncertainties . . . . . . . . . . . 104

6.2 Limit Setting . . . . . . . . . . . . . . . . 106

6.2 .1 Overview . . . . . . . . . . . . . 106

6.2 .2 Log-Liklihood Ratios . . . . . . . . . . . . . . . . . 107

6.2 .3 Cross Section Limits . . . . . . . . . . . . . . . . . 108

6.2 .4 Systematic Effects . . . . . . . . . . . . . 110

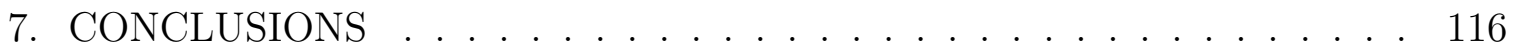

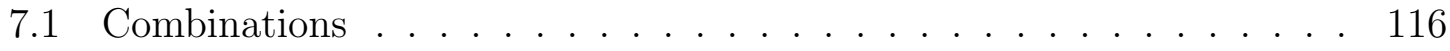

$7.1 .1 \quad \mu \tau j j$ and $e \tau j j \ldots \ldots \ldots \ldots \ldots \ldots \ldots$ 
7.1.2 DØ and CDF Combinations .............. . . 118

7.2 Discussion . . . . . . . . . . . . . . . . . . . 123

7.3 Final Summary . . . . . . . . . . . . . . . . . 125

Appendix

A. EMinclusive Dataset . . . . . . . . . . . . . . . . . . . . . . . 129

B. MC Generation Tables . . . . . . . . . . . . . . . . . . 131

C. Jet Shape Systematic Modeling . . . . . . . . . . . . . . . 136

D. Epilogue . . . . . . . . . . . . . . . . . . . . 142

Bibliography . . . . . . . . . . . . . . . . . . . 145

BIOGRAPHICAL STATEMENT . . . . . . . . . . . . . . . . 150 


\section{LIST OF ILLUSTRATIONS}

Figure

1.1 Example potential $V(\phi)=-\frac{1}{2} \mu^{2}\left(\phi^{*} \phi\right)+\frac{1}{4} \lambda\left(\phi^{*} \phi\right)^{2}$ showing (a) an unbroken and (b) a broken symmetry . . . . . . . . . . . 8

1.2 Restrictions of possible Higgs mass from $W$ boson and $t$-quark mass measurements. The overlap between the red oval and the green stripe represent the last allowed values for the SM Higgs . . . . . . . . . .

1.3 Theoretical restrictions for the Higgs mass plotted as a function of $\Lambda$, the energy scale . . . . . . . . . . . . . . . .

1.4 Higgs production mode cross sections at $\sqrt{s}=1.96 \mathrm{TeV}$ for $p \bar{p}$ collisions. Gluon fusion is the dominant process, however most searches rely most heavily on associated production to help improve background rejection

1.5 Higgs branching ratios. The primary decays considered at the Tevatron are $H \rightarrow b \bar{b}, W W, \tau \tau$ and $\gamma \gamma$. The $c \bar{c}$ and $g g$ modes are overrun by jet backgrounds, and the $Z Z$ mode does not have enough statistics to

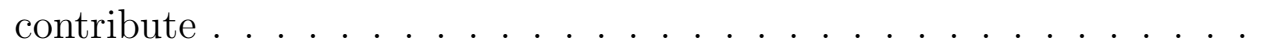

1.6 Branching fractions for $\tau \tau \rightarrow \tau_{x} \tau_{x}$. The most probable decay is for both taus to decay hadronically. With equal probability one will decay hadronically and the other leptonically. The three lepton only decays contribute only $9 \%$ combined . . . . . . . . . . . . . . 
1.7 Feynman diagrams for the three production modes considered in this analysis: (a) associated production, (b) gluon fusion, (c) vector boson fusion. In any production mode the Higgs can decay into a pair of taus

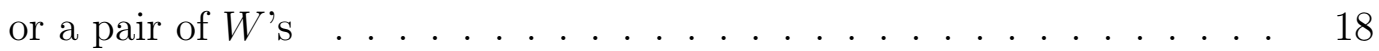

1.8 Fractional expected signal yields in the final selection. $\tau \tau$ decays are shown in solid lines and $W W$ decays in dashed lines. The contamination of $W W$ signals in the low mass region is less than the contamination from $\tau \tau$ signals in the high mass region $\ldots \ldots \ldots \ldots$

2.1 Schematic view of the Fermilab accelerator campus. The Tevatron has a radium of $1 \mathrm{~km}$, and houses both general purpose detectors: CDF and

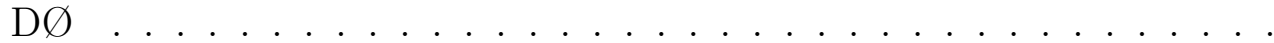

2.2 Schematic side view of the $\mathrm{D} \varnothing$ detector showing (starting closest to the beam) the tracker, calorimeter and muon system $\ldots \ldots \ldots \ldots$

2.3 Schematic view of the inner detector designed to measure charged particles tracks and momenta . . . . . . . . . . . 26

2.4 Schematic view of the inner silicon tracking system showing six barrels and 14 disks. The majority of the SMT is designed to be perpendicular to the beam direction $\ldots \ldots \ldots \ldots \ldots \ldots \ldots$

2.5 Schematic end view of the silicon tracking system with overlapping layers to maximize the number of hits per track . . . . . . . . 29

2.6 Schematic end view of the CFT and waveguides that guide the signals underneath the detector for processing . . . . . . . . . 30

2.7 Approximate field lines due to the solenoid and toroid magnets . . . . 31 
2.8 Schematic view of the calorimeter. It is more finely segmented in the electromagnetic portion closer to the beam to provide better resolution. The absorber material is mostly depleted uranium, and the active material is liquid $\operatorname{argon} \ldots \ldots . \ldots 32$

2.9 Schematic view of one calorimeter cell. Free electrons from the ionized argon gas are accelerated over a potential difference of $2 \mathrm{kV}$ and collected on the signal board . . . . . . . . . . . . . . . .

2.10 One quadrant of the calorimeter. The finer segmentation is located nearer the beam. There is only minimal EM calorimeter coverage in the region $1.1<\eta<1.3 \ldots \ldots \ldots$. . . . . . . . . . . . . 34

2.11 Schematic exploded view of the three layered muon system. The toroidal magnet is not pictured . . . . . . . . . . . . . .

2.12 Schematic drawing showing the position of the luminosity monitors $\pm 140 \mathrm{~cm}$ up and down stream from the interaction point . . . . . . 36

2.13 Schematic view of the trigger system shows each level, and it's integration into the data acquisition system . . . . . . . . . . . . . 38

3.1 (a), (b) and (c) sample $\mathrm{NN}_{\tau}$ distributions by type and (d) $\mathrm{NN}_{\tau / e}$. The contribution from real taus from $Z \rightarrow \tau \tau$ is concentrated towards +1 in each distribution . . . . . . . . . . . . . . . . . . 46

3.2 Tau ID efficiency curve for type 2 taus, (a) old $\mathrm{NN}_{\tau}$ and (b) new $\mathrm{NN}_{\tau}$. The green and red lines show the efficiency for different $p_{T}$ selections used to calculate systematic uncertainties . . . . . . . . . .

4.1 Percent of data loss by detector subsystem. Partial outages in the calorimeter are the major source of loss . . . . . . . . . . . 
4.2 Electron $p_{T}$ trigger efficiency curves for the EJetsOR trigger in the (a) central calorimeter and (b) the end calorimeter for slightly different trigger lists. The tag-and-probe method is more difficult in the end calorimeter and causes a slight drop in efficiency . . . . . . . . . . . 54

4.3 Electron $p_{T}$ for three different triggers (a) inclusive, (b) SingleEM and (c) EJetsOR. The trigger turn-on is visible in (b) and (c) and ultimately (c) was chosen as a compromise between increased yield and quality modeling (see Fig. 4.4 for the legend) . . . . . . . . . . . . . . .

4.4 Legend for all plots in this paper. Signals are shown in a solid black lines multiplied by $250 \ldots \ldots$. . . . . . . . . . . . . . 55

4.5 Comparing the shape similarity of signal (black line) with $Z \rightarrow \tau \tau$ background (blue) in the tau transverse momentum distribution . . . . 56

4.6 W+jets control sample plots used to check the modeling of this background. Most variables show decent agreement . . . . . . . . . . . 58

4.7 Feynman diagrams for four of the background processes. (a) $W+$ jets, (b) $t \bar{t}$ and (c) multi-jet and (d) di-boson . . . . . . . . . . . . 59

4.8 The $\eta^{\tau}$ distributions (a) without the type 1 ICR removal and (b) with the removal. Electrons entering this region of the detector are recon-

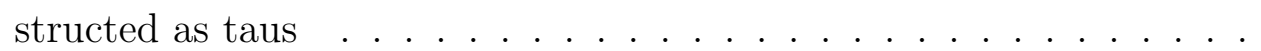

4.9 MET significance distributions (a) without the imposition of the $\mathcal{S}$ selection for Run IIa and Run IIb combined, and with the $\mathcal{S}$ selection for (b) Run IIa and (c) Run IIb. Missing energy actually resulting from neutrinos peaks around 6, while missing energy from detector mismeasurement peaks at $0 \ldots \ldots \ldots \ldots$ 
4.10 Distributions of the ratio of OS to SS events in the MJ enriched sample, as function of (top) $p_{T}^{e}$, (middle) $p_{T}^{\tau}$ and (bottom) $p_{T}^{(1)}$ for different $\tau$ types. The red line shows a flat fit and therefore no $\rho$ dependence within the good statistics region of each distribution . . . . . . . . . . 68

4.11 Distributions of the ratio of OS to SS events in the MJ enriched sample, as function of (top) $\eta_{e}$, (middle) $\eta_{\tau}$ and (bottom) $\eta^{(1)}$ for different $\tau$ types. The red line shows a flat fit and therefore no $\rho$ dependence within the good statistics region of each distribution . . . . . . .

5.1 An example decision tree showing the splitting of the sample into signallike and background-like nodes . . . . . . . . . . . . . . . 73

5.2 Transverse momentum distributions for various objects, and the combined $p_{T}$ of the tau, electron and $\mathbb{E}_{T}$. The signals are shown multiplied by $250 \ldots \ldots \ldots \ldots \ldots \ldots \ldots$

5.3 $\eta$ distributions for various objects, and $\Delta R$ between the jets. The signals are shown multiplied by 250 . . . . . . . . . . . . .

$5.4 p_{T}$ distributions for various object combinations. The signals are shown multiplied by $250 \ldots \ldots$. . . . . . . . . . . . . . . . 80

5.5 $\Delta \phi$ minimum and maximum distributions for various object combinations. The signals are shown multiplied by 250 . . . . . . . . . . 81

5.6 Transverse mass distributions. The signals are shown multiplied by $250 \quad 82$

5.7 Invariant mass distributions of the two jets, electron + tau + jets, $\tau \tau$, and $W W$. The signals are shown multiplied by $250 \ldots \ldots$. . . . . . 83

5.8 The number of physical solutions, $\mathbb{E}_{T}$ and $\mathcal{S}$ distributions. The signals are shown multiplied by $250 \ldots \ldots$. . . . . . . . . . . . 84

5.9 Electron MVA, missing energy $\Delta \phi$, missing energy asymmetry, and $\cos \theta^{*}$. The signals are shown multiplied by $250 \ldots \ldots$. . . . . . 
5.10 Schematic of the final MVA procedure. The final sample will be split, each sample will develop its own limits, and the two subsamples will be recombined to set the final limits . . . . . . . . . . . . . .

5.11 BDT trained to discriminate $\tau \tau$ signals from $W W$ signals using all signal events between 105 and $150 \mathrm{GeV}$. For this plot only, the sum of $\tau \tau$ signals is shown in the solid line and the sum of all $W W$ signals is shown in the dotted line. The red line shows the separation of the two

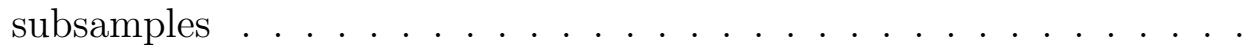

5.12 Comparison of limits using the $\mathrm{BDT}_{\mathrm{TW}}$ method. (a) Combination of the subsamples and (b) no separation. The expected limit improves by $\sim 10 \%$ when using the $\mathrm{BDT}_{\mathrm{TW}} \ldots \ldots . \ldots 90$

5.13 The limit and LLR distributions for two early BDT techniques, (a), (b) training at each mass point; (c), (d) training at each mass point with $50 \%$ contribution from the point and $25 \%$ from $m_{H} \pm 5 \mathrm{GeV}$. The averaging technique only marginally helped reduce the oscillatory behaviors. Compare to Fig. 6.3 . . . . . . . . . . . . . .

$5.14 m_{H}$ distributions used for BDT training for (a) T subsample and (b) W subsample. The background (red) was normalized to the signal (blue) to not allow the BDT artificially good separation between the two . . .

5.15 BDT outputs for $m_{H}=105 \mathrm{GeV}$. (a) The T subsample output, (b) the W subsample output. The signals are shown multiplied by 250 . . . .

5.16 BDT outputs for $m_{H}=110 \mathrm{GeV}$. (a) The T subsample output, (b) the W subsample output. The signals are shown multiplied by 250 . . . . 94

5.17 BDT outputs for $m_{H}=115 \mathrm{GeV}$. (a) The T subsample output, (b) the W subsample output. The signals are shown multiplied by 250 . . . 
5.18 BDT outputs for $m_{H}=120 \mathrm{GeV}$. (a) The T subsample output, (b) the W subsample output. The signals are shown multiplied by $250 \ldots 95$

5.19 BDT outputs for $m_{H}=125 \mathrm{GeV}$. (a) The T subsample output, (b) the W subsample output. The signals are shown multiplied by $250 \ldots 96$

5.20 BDT outputs for $m_{H}=130 \mathrm{GeV}$. (a) The T subsample output, (b) the W subsample output. The signals are shown multiplied by $250 \ldots 6$

5.21 BDT outputs for $m_{H}=135 \mathrm{GeV}$. (a) The T subsample output, (b) the W subsample output. The signals are shown multiplied by $250 \ldots 97$

5.22 BDT outputs for $m_{H}=140 \mathrm{GeV}$. (a) The T subsample output, (b) the W subsample output. The signals are shown multiplied by $250 \ldots 7$

5.23 BDT outputs for $m_{H}=145 \mathrm{GeV}$. (a) The T subsample output, (b) the W subsample output. The signals are shown multiplied by $250 \ldots 98$

5.24 BDT outputs for $m_{H}=150 \mathrm{GeV}$. (a) The T subsample output, (b) the W subsample output. The signals are shown multiplied by $250 \ldots 98$

5.25 Comparison of adjacent mass point BDT outputs: [(a), (b), (e) and (f)] with the Global BDT and [(c), (d), (g) and (h)] with individual mass point trainings. The shape change of the signal and background without the Global BDT from $m_{H}=115$ to $120 \mathrm{GeV}$ (140 to 145 ) in the $\mathrm{T}$ (W) subsample is one example of the large variability in adjacent mass point training $\ldots \ldots 100$

6.1 Normalized $\delta \mathrm{BDT}_{ \pm 1 \sigma}$ outputs of the jet ID and reconstruction efficiencies, for (top row) the T subsample at $115 \mathrm{GeV}$ and for (bottom row) the $\mathrm{W}$ subsample at $115 \mathrm{GeV}$. The signals, $Z+$ jets and $W+$ jets + $t \bar{t}$ backgrounds are shown in the left, middle and right columns respectively 111 
6.2 Various plots to quantify the effect of systematic uncertainties. (a) $\sigma$ value to achieve the best fit of background to data, (b) effect of removing any single uncertainty on the best fit parameter $\Delta \chi^{2}$ and (c) the effect of the total uncertainty by BDT output bin . . . . . . . . . . .

6.3 LLR for (a) the $\mathrm{T}$ subsample, (b) the $\mathrm{W}$ subsample and (c) the $\mathrm{T}$ and $\mathrm{W}$ subsamples combined as a function of Higgs mass, for expected background only (black dotted line), expected signal + background (red dotted line) hypotheses, and the observed values (solid black line). The $\pm 1 \sigma$ and $\pm 2 \sigma$ variations from the expected background only hypothesis are shown in green and yellow bands respectively . . . . . . . . . .

6.4 Cross section limits for (a) the T subsample, (b) the W subsample and (c) the $\mathrm{T}$ and $\mathrm{W}$ subsamples combined as a function of Higgs mass, for expected background only (dashed red line), and the observed values (black line) . . . . . . . . . . . . . . . . .

6.5 Cross section limits and LLR distributions for (a), (b) the combined subsamples without systematic uncertainties; and (c), (d) the combined subsamples with the systematic uncertainties as a function of Higgs mass 115

7.1 Limits for the combined $\mu \tau j j$ and $e \tau j j$ analysis for (a) the T subsample and (b) the $\mathrm{W}$ subsample as a function of Higgs mass, for expected background only (red dotted line), and the observed values (solid black line $\ldots \ldots \ldots \ldots \ldots 118$

7.2 Limit plot for the combined $\mu \tau j j$ and $e \tau j j$ analysis as a function of Higgs mass. The relatively flat sensitivity can be attributed to the use of two Higgs decay modes . . . . . . . . . . . . . . . . . . . . . . . . 119 
7.3 LLR for the combined $\mu \tau j j$ and $e \tau j j$ analysis for (a) the T subsample and (b) the W subsample as a function of Higgs mass, for expected background only (black dotted line), expected signal + background (red dotted line) hypotheses, and the observed values (solid black line). The $\pm 1 \sigma$ and $\pm 2 \sigma$ variations from the expected background only hypothesis are shown in green and yellow bands respectively . . . . . . . . . .

7.4 LLR for the combined $\mu \tau j j$ and $e \tau j j$ analysis as a function of Higgs mass, for expected background only (black dotted line), expected signal + background (red dotted line) hypotheses, and the observed values (solid black line). The $\pm 1 \sigma$ and $\pm 2 \sigma$ variations from the expected background only hypothesis are shown in green and yellow bands respectively 120

7.5 The combined CDF and D $\varnothing$ limits by channel: (a) $\gamma \gamma$, (b) $W W$ and (c) $b \bar{b}$. A broad excess is seen in the $b \bar{b}$ channel . . . . . . . . . . . . 121

7.6 Higgs exclusion for the combined CDF and DØ experiments as a function of Higgs mass. The shaded regions indicate where the Higgs boson has been excluded by previous experiments. A significant excess in the region $115<m_{H}<140 \mathrm{GeV}$ gives evidence of a new boson $\ldots \ldots$. . 122

7.7 LLR for the combined CDF and D $\varnothing$ Experiments as a function of Higgs mass. In the region $115<m_{H}<140 \mathrm{GeV}$ the data agree well with the signal + background hypothesis $\ldots \ldots \ldots \ldots \ldots \ldots$

7.8 The Tevatron combined LLR plots from 2007-2012. In most plots the data follows the signal + background prediction in the region 110-140 $\mathrm{GeV} \ldots \ldots \ldots \ldots \ldots \ldots \ldots \ldots \ldots \ldots$

7.9 The best fit signal strength for the Tevatron combination (black line) and for each major decay mode (black squares). The green band is the $1 \sigma$ uncertainty on the average $\ldots \ldots \ldots \ldots \ldots$ 
7.10 Comparison of (a) the previous and (b) the new e $\tau j j$ limits. The Global BDT method achieves the smoothly varying limit and all the various improvements combined to give more that $50 \%$ improvement in expected limits. Note the different $\mathrm{x}$ axis scales . . . . . . . . . . 127

7.11 Comparison of (a) the previous and (b) the new $\ell \tau j j$ limits. There is an overall $\sim 20 \%$ improvement, as well as a much more smoothly varying distribution. Note the different x-axis scales . . . . . . . . . . 128 


\section{LIST OF TABLES}

Table

Page

1.1 Fundamental fermions of the SM. Masses are given in $\mathrm{GeV} / \mathrm{c}^{2}$, charges in units of $e \ldots \ldots \ldots \ldots \ldots \ldots \ldots$

1.2 Fundamental bosons of the SM. Masses are given in $\mathrm{GeV} / c^{2}$, charges

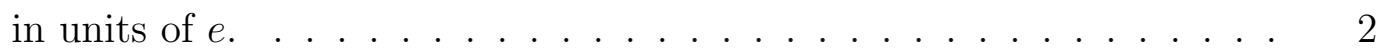

1.3 Leptonic and hadronic branching ratios for the tau lepton. . . . . . . 3

1.4 Production and decay mode summary. . . . . . . . . . . . . . . . 18

2.1 Summary of approximate trigger rates. . . . . . . . . . . . . . . . 37

3.1 Detector driven tau decay types, with branching ratios and detector signatures. . . . . . . . . . . . . . . . . . . . 44

3.2 Tau ID event yields and uncertainty for the old (left) and new (right) $\mathrm{NN}_{\tau}$ for Run IIb3+4. . . . . . . . . . . . . . . . . . . . . 48

4.1 Number of events, purity (fraction of events estimated to be MJ) and scale factors, by tau type for the Run IIa MJ enriched sample. . . . . .

4.2 Events retained after successive cuts for the Run IIa portion of the analysis, for data and the expected SM and MJ backgrounds. The signal yields are quoted for $m_{H}=125 \mathrm{GeV}$. . . . . . . . . . . . .

4.3 Events retained after successive cuts for the Run IIb portion of the analysis, for data and the expected SM and MJ backgrounds. The signal yields are quoted for $m_{H}=125 \mathrm{GeV}$. . . . . . . . . . . . 
4.4 After preselection in the Run IIa and IIb combined, the number of data and the expected SM and MJ backgrounds for both the total and individual tau types. The signal yields are quoted for $m_{H}=125 \mathrm{GeV}$.

4.5 Number of events for each signal/decay channel expected in the final selection in the T subsample as a function of Higgs boson mass. . . . . 70

4.6 Number of events for each signal/decay channel expected in the final selection in the $\mathrm{W}$ subsample as a function of Higgs boson mass. . . . 71

4.7 Comparison of the contributions from each background by percent for the previous and current results. . . . . . . . . . . . . .

5.1 Effect of different selections $(-0.8,-0.6,-0.5)$ of $\mathrm{BDT}_{\mathrm{TW}}$ on background yields and purity. . . . . . . . . . . . . .

5.2 Expected limits for the $\mathrm{T}$ and $\mathrm{W}$ subsamples using the di-jet mass as the final discriminant. There are no signs of oscillation. . . . . . . .

5.3 Highest BDT value event comparison of Global BDT versus nominal. The Global BDT values remain essentially the same, while the nominal method can have severe fluctuations. . . . . . . . . . . . . . . .

6.1 Systematic uncertainties in percent. The range of jet shape uncertainties is shown separately for the $\mathrm{T}$ and $\mathrm{W}$ subsamples. . . . . . . . . 105

6.2 Ratio of the expected and observed 95\% C.L. limits to the SM expectations. . . . . . . . . . . . . . . . . . . 109

7.1 Final limits obtained for the combined Run II $\mu \tau j j$ and $e \tau j j$ analysis. 117

7.2 Summary of all the major improvements completed for this analysis and their approximate effect on the overall limit. . . . . . . . . 126

7.3 Comparison of limits with the previous analysis using $4.3 \mathrm{fb}^{-1}$ and the percent improvement of the expected limit. The doubling of luminosity only accounts for $\sim 30 \%$ of the improvement. 


\section{CHAPTER 1}

\section{THEORY}

\subsection{Introduction}

The standard model (SM) of particle physics ([1]-[2]) is a fantastically accurate theory describing the fundamental interactions that give structure to our Universe. Developed over several decades and by innumerable physicists it has been validated many times over. It prescribes three families, or generations, of fundamental particles each containing four fermions (1/2 - integer spin): two quarks, and two leptons. Successive generations are manifested as heavier versions of the previous. Table 1.1 summarizes each particles mass, charge and spin. Every fermion has an anti-particle partner with opposite charge and lepton number. The SM also describes three fundamental forces, electromagnetic, weak, and strong. These forces are mediated by 12 bosons (integer spin): photon (electromagnetic), $W^{ \pm}$and $Z^{0}$ (weak), and 8 gluons (strong). Table 1.2 summarizes the force carrying particles

Table 1.1. Fundamental fermions of the SM. Masses are given in $\mathrm{GeV} / c^{2}$, charges in units of $e$.

\begin{tabular}{cccc|cccc}
\hline \hline Particle & Mass & Charge & Spin & Particle & Mass & Charge & Spin \\
\hline electron & 0.000511 & 1 & $1 / 2$ & up & 0.003 & $2 / 3$ & $1 / 2$ \\
$\nu_{e}$ & $<2.2 \times 10^{-9}$ & 1 & $1 / 2$ & down & 0.006 & $-1 / 3$ & $1 / 2$ \\
muon & 0.106 & 1 & $1 / 2$ & strange & 0.1 & $2 / 3$ & $1 / 2$ \\
$\nu_{e}$ & $<170 \times 10^{-6}$ & 1 & $1 / 2$ & charm & 1.27 & $-1 / 3$ & $1 / 2$ \\
tau & 1.78 & 1 & $1 / 2$ & bottom & 4.19 & $-1 / 3$ & $1 / 2$ \\
$\nu_{\tau}$ & $<15.5 \times 10^{-3}$ & 1 & $1 / 2$ & top & 172 & $2 / 3$ & $1 / 2$ \\
\hline \hline
\end{tabular}


Table 1.2. Fundamental bosons of the SM. Masses are given in $\mathrm{GeV} / c^{2}$, charges in units of $e$.

\begin{tabular}{cccc}
\hline \hline Particle & Mass & Charge & Spin \\
\hline$\gamma$ & 0 & 0 & 1 \\
$W^{+}$ & 80.4 & 1 & 1 \\
$W^{-}$ & 80.4 & -1 & 1 \\
$Z$ & 91.2 & 0 & 1 \\
$g$ & 0 & 0 & 1 \\
\hline \hline
\end{tabular}

The SM correctly predicted the existence of top and bottom quarks, as well as the mass of the $W$ and $Z$ bosons. Measurements of $\alpha$, the fine structure constant, have been made to better than one part per trillion using predictions from quantum electrodynamics (QED). However, for all of its success the SM is incomplete. The three generation structure of the SM is not predicted, and no mechanism for its presence is currently known. Evidence from neutrino oscillation experiments prove that neutrinos have mass, whereas they are assumed to be massless in the SM. Also, the amount of CP-violation is insufficient to explain the currently observed matterantimatter asymmetry.

Most importantly all particle masses are experimentally determined parameters and are not predicted. Additionally, the vast range of quark masses, spanning six orders of magnitude is a peculiar arrangement chosen by the Universe, and unexplained in the SM. These issues alone will drive particle physics forward in the future, but with history serving as a reminder, a world completely foreign to our understanding likely awaits.

In this paper the high energy physics short hand for units are used. Masses, momenta, and energy are all quoted in electronvolts where the correct power of $c$ is implied: $m=\left[\mathrm{GeV} / c^{2}\right], p=[\mathrm{GeV} / c]$, and $E=[\mathrm{GeV}]$. 
Table 1.3. Leptonic and hadronic branching ratios for the tau lepton.

\begin{tabular}{cc}
\hline \hline$\tau$ type & Branching Ratio \\
\hline$\tau^{ \pm} \rightarrow e^{ \pm} \bar{\nu}_{e} \nu_{\tau}$ & $17.85 \%$ \\
$\tau^{ \pm} \rightarrow \mu^{ \pm} \bar{\nu}_{\mu} \nu_{\tau}$ & $17.36 \%$ \\
$\tau^{ \pm} \rightarrow h^{ \pm} \nu_{\tau}$ & $64.79 \%$ \\
\hline \hline
\end{tabular}

\subsection{The Tau Lepton}

The tau lepton is integral to this analysis and a complete understanding of its properties is necessary. The first indirect evidence for the tau was found at SLAC in 1975, through the process $e^{+}+e^{-} \rightarrow e^{ \pm}+\mu^{\mp}+\geq 2$ particles [3]. The new particle was given the name $\tau \rho \iota \tau o \nu$, or "triton" the Greek word for third, since it was the third charged lepton to be discovered [4].

The tau has a mass of $1.777 \mathrm{GeV}$, roughly 3500 times the mass of its first generation cousin, the electron, and has quite a short lifetime with $c \tau=87.11 \mu \mathrm{m}$. As with the muon, the tau can decay into lighter (less massive) leptons while conserving lepton number. Consider the simple leptonic decay of a tau:

$$
\tau^{ \pm} \rightarrow e^{ \pm} \bar{\nu}_{e} \nu_{\tau}
$$

First, charge is conserved via the electron charge. Second, lepton number is conserved by the presence of the tau neutrino, and the anti-electron neutrino. The same process is valid if the $e$ is replaced by a $\mu$ and $\bar{\nu}_{e}$ with $\bar{\nu}_{\mu}$. Leptonic decays must therefore always carry at least 2 neutrinos. The tau is unique among leptons because its comparatively large mass allows for decays into hadrons containing $u, d, c$ or $s$ quarks through the process $\tau^{ \pm} \rightarrow h^{ \pm} \nu_{\tau}$, where $h^{ \pm}$is any hadron. Typically there are also one or more neutral hadron ( $\operatorname{such}$ as $\pi^{0}$ ). In both cases the decay is mediated by a $W$ boson. Table 1.3 summarizes the tau branching ratios into leptons and hadrons. 


\subsection{The Standard Model}

The SM is a theoretical framework based on quantum field theory that describes the fundamental constituents of matter and their interactions. It is based on the product of (local) gauge symmetries that correspond to the strong, $\mathrm{SU}(3)_{C}$, and the unified electromagnetic and weak interactions $\mathrm{SU}(2)_{L} \times \mathrm{U}(1)_{Y}$. The Lagrangian for the SM contains all terms which are invariant under the local gauge transformations corresponding to these groups. Consider the basic example of the Dirac free Lagrangian (Eq. 1.2) and how it behaves under the transformation given in Eq. 1.3:1

$$
\begin{gathered}
\mathcal{L}_{D}=\bar{\psi}\left(i \gamma^{\mu} \partial_{\mu}-m\right) \psi \\
\psi \rightarrow e^{i \theta(x)} \psi
\end{gathered}
$$

where $\theta$ is a real number and a function of $x^{\mu}$ that acts as a phase transformation. Under this transformation the Dirac free Lagrangian will acquire an extra term, hence

$$
\mathcal{L}_{D} \rightarrow \mathcal{L}_{D}-\left(\partial_{\mu} \theta\right) \bar{\psi} \gamma^{\mu} \psi
$$

It is therefore necessary to introduce a gauge field, $A_{\mu}$, to the Lagrangian to absorb the additional term and maintain the gauge invariance. The new field couples to the original field through a term in the Lagrangian that goes as $q\left(\bar{\psi} \gamma^{\mu} \psi\right) A_{\mu}{ }^{2}$ has a free term proportional to $m_{A} A^{\nu} A_{\nu}$ and transforms under the local gauge as

$$
A_{\mu} \rightarrow A_{\mu}-\frac{\hbar c}{q} \theta(x)
$$

\footnotetext{
${ }^{1}$ The theoretical arguments that follow are based of those found in [5] and [6]. ${ }^{2} q$ is the charge of the particle.
} 
However, the terms in a Lagrangian involving $A^{\nu} A_{\nu}$ are not invariant under the transformation in Eq. 1.5 which necessarily means the gauge field must be massless, $m_{A}=0$. The preservation of invariance can be similarly be achieved by replacing the partial derivative in the free Lagrangian (Eq. 1.2) with the covariant derivative defined as

$$
\mathcal{D}_{\mu} \equiv \partial_{\mu}+i \frac{q}{\hbar c} A_{\mu}
$$

To write a complete Lagrangian, a kinetic term of the gauge field must be added to Eq. 1.2

$$
\mathcal{L}_{K E}=-\frac{1}{4} F_{\mu \nu} F^{\mu \nu}
$$

where $F^{\mu \nu}=\partial^{\mu} A^{\nu}-\partial^{\nu} A^{\mu}$. Requiring the $\mathrm{U}(1)_{Y}$ local gauge invariance of the form of Eq. 1.3 transforms the Dirac free Lagrangian into an interacting one, and fully describes the interactions of photons and electrons, the theory of quantum electrodynamics, QED, hence

$$
\mathcal{L}_{Q E D}=\bar{\psi}\left(i \gamma^{\mu} \partial_{\mu}-m\right) \psi-\frac{1}{4} F_{\mu \nu} F^{\mu \nu}-\frac{q}{\hbar c}\left(\bar{\psi} \gamma^{\mu} \psi\right) A_{\mu}
$$

By changing the form of the local invariance, this procedure can be generalized to exploit higher order symmetries, such as the weak force under $\mathrm{SU}(2)_{L}$. For instance, consider the transformation

$$
\psi \rightarrow e^{i \boldsymbol{\sigma} \cdot \boldsymbol{a}} \psi
$$

where $\boldsymbol{\sigma}$ is one of the three, $2 \times 2$ Pauli matrices, and $\boldsymbol{a}$ is a matrix of real numbers. It is necessary to then introduce 3 massless gauge fields $\mathbf{A}_{\mu}$. From observation we know these three bosons, which are identified as the $W^{ \pm}$and $Z^{0}$ particles, to be 
massive and thus the electroweak symmetry of $\mathrm{SU}(2)_{L} \times \mathrm{U}(1)_{Y}$ must be broken (see Section 1.3.1).

Quantum chromodynamics (QCD or the strong force) is built in a similar way as the weak force except it is based on the $\mathrm{SU}(3)_{C}$ symmetry. The gauge invariance becomes

$$
\psi \rightarrow e^{i\left(\theta I_{3}+\boldsymbol{\lambda} \cdot \boldsymbol{a}\right)}
$$

where $I_{3}$ is the $3 \times 3$ identity matrix, $\boldsymbol{\lambda}$ are the Gell-Mann matrices, and $\boldsymbol{a}$ is again a matrix of real numbers. There are now 8 gauge fields, which are used in the covariant derivative definition:

$$
\mathcal{D}_{\mu} \equiv \partial_{\mu}+i \frac{q}{\hbar c} \boldsymbol{\lambda} \cdot \mathbf{A}_{\mu}
$$

The final QCD Lagrangian describes three equal mass Dirac fields (three colors of any given quark type) that interact with eight massless vector fields representing all the color combination of gluons.

QCD has the peculiar property of asymptotic freedom, a result of the antiscreening effect of gluon loops in higher order Feynman processes. The QCD coupling constant $\alpha_{s}$ is then dependent on the energy scale of the interaction and as a result the strong force becomes quite weak at very short distances. At the energies considered here, $\mathrm{SU}(3)_{C}$ symmetry is the only symmetry not broken in the SM, hence all eight gluons remain massless, and have only two polarization states.

\subsubsection{Symmetry Breaking - The Higgs Mechanism}

The SM is fundamentally built on symmetries. When some of these symmetries are broken they create the widely varying particle masses that are observed. One 
of the most common means of breaking gauge symmetries is through the so-called Higgs mechanism. In this approach, a fundamental scalar field (which transforms in a particular way under the gauge symmetries) is introduced. If this scalar field acquires a non-zero expectation value (Eq. 1.12), it is possible that one or more of the gauge symmetries can be broken and the corresponding gauge fields can acquire mass.

$$
\langle\hat{A}|\phi| \hat{A}\rangle \neq 0
$$

To demonstrate this property, consider introducing a complex scalar field into a theory containing a single $\mathrm{U}(1)_{Y}$ gauge field:

$$
\begin{gathered}
\phi=\phi_{1}+i \phi_{2} \\
\phi^{*} \phi=\phi_{1}^{2}+\phi_{2}^{2}
\end{gathered}
$$

The locally gauge invariant Lagrangian, with covariant derivatives becomes

$$
\mathcal{L}=\frac{1}{2} \mathcal{D}_{\mu} \phi^{*} \mathcal{D}^{\mu} \phi-\frac{1}{4} F^{\mu \nu} F_{\mu \nu}-V(\phi)
$$

where the potential is $V(\phi)=-\frac{1}{2} \mu^{2}\left(\phi^{*} \phi\right)+\frac{1}{4} \lambda\left(\phi^{*} \phi\right)^{2}$. If $\mu^{2}>0$ the symmetry is preserved (Fig. 1.1 (a)), but if $\mu^{2}<0$ the symmetry is broken and the infamous "mexican hat" potential is uncovered (Fig. 1.1 (b) [7]). Using the condition $\frac{\partial V}{\partial \phi}=0$ to find the local minima of $V(\phi)$, it is found that

$$
\left|\phi_{1 \min }\right|^{2}+\left|\phi_{2 \min }\right|^{2}=\frac{\mu^{2}}{\lambda} \equiv v^{2}
$$

Without loss of generality the non-zero vacuum state is chosen along the $\phi_{1}$-axis

$$
\langle\phi\rangle=\left(\begin{array}{l}
v \\
0
\end{array}\right)
$$




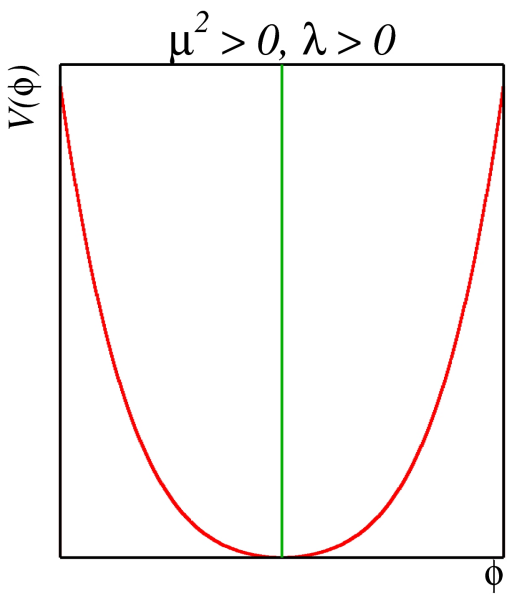

(a)

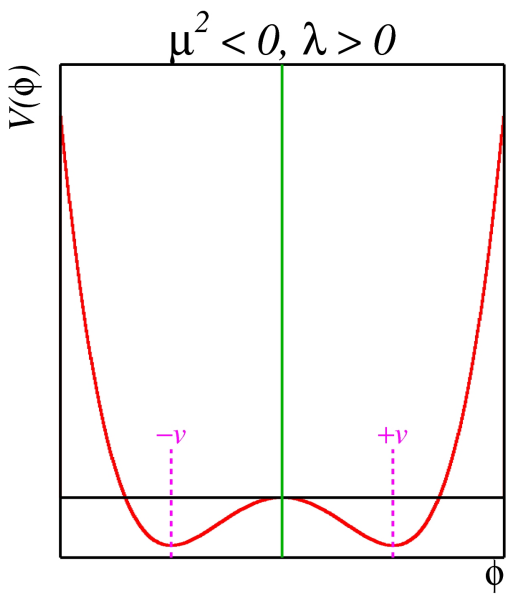

(b)

Figure 1.1. Example potential $V(\phi)=-\frac{1}{2} \mu^{2}\left(\phi^{*} \phi\right)+\frac{1}{4} \lambda\left(\phi^{*} \phi\right)^{2}$ showing (a) an unbroken and (b) a broken symmetry.

The selection of a vacuum expectation value effectively chooses a direction in the potential and breaks the symmetry. Spontaneous symmetry breaking typically leads to massless (and unphysical) Goldstone bosons. However, gauge transformations allow the freedom to choose the gauge parameter such that only physical fields appear in the Lagrangian. This is called unitary gauge and is given by the choice

$$
\theta=-\tan ^{-1}\left(\phi_{2} / \phi_{1}\right)
$$

and the final Lagrangian (with $h$ as the scalar Higgs field ${ }^{3}$ ) is

$$
\begin{gathered}
\mathcal{L}=\frac{1}{2}\left(\partial_{\mu} h\right)^{2}-\mu^{2} h^{2}-\frac{1}{4} F^{\mu \nu} F_{\mu \nu}+\frac{1}{2}(q v)^{2} A_{\mu} A^{\mu} \\
+\frac{1}{2} q^{2} h^{2} A_{\mu} A^{\mu}+\left(v q^{2}\right) h A_{\mu} A^{\mu}-\sqrt{\lambda} \mu h^{3}-\frac{1}{4} \lambda h^{4}
\end{gathered}
$$

The gauge field introduced to keep the local invariance was originally massless, but when the vacuum symmetry is broken it acquired a mass. This is the Higgs

\footnotetext{
${ }^{3}$ When the symmetry is broken the field becomes $\phi \rightarrow v+h$
} 
mechanism ([8]-[9]) that gives the $W^{ \pm}$and the $Z^{0}$ their mass. This provides a plausible explanation for how the vector bosons have acquired mass, but also hypothesizes that one other boson should exist, the Higgs boson.

Not only does the Higgs mechanism give mass to the vector bosons, but it is also responsible for the mass of the quarks and leptons. Consider the $\mathrm{SU}(2)_{L}$ doublet and the right handed singlets

$$
\psi_{e}=\left(\begin{array}{c}
\nu_{e} \\
e^{-}
\end{array}\right) \text {and } e_{R}^{-}
$$

Of course in the SM all neutrinos are left-handed and all anti-neutrinos are righthanded. In accordance with the gauge symmetries of the theory, it is possible to write down interactions between the SM fermions and the Higgs field which are invariant under the gauge transformations. The Lagrangian for leptons goes as

$$
\mathcal{L}_{\mathrm{Y}}=g_{e} \bar{\psi} \phi e_{R}+g_{e} \bar{e}_{R} \phi^{\dagger} \psi
$$

where $g_{e}$ is called the Yukawa coupling, and $\phi$ is the complex scalar Higgs field from earlier. When the symmetry of $\phi$ is broken two terms arise ${ }^{4}$

$$
\mathcal{L}_{\mathrm{Y}}=\left(g_{e} v\right) \bar{\psi} e_{R}+\left(g_{e} h\right) \bar{\psi} e_{R}+\left(g_{e} v\right) \bar{e}_{R} \psi+\left(g_{e} h\right) \bar{e}_{R} \psi+\text { h.c. }
$$

The first term describes a particle with mass $m=g_{e} v$, and the second describes the coupling of the Higgs field to the lepton. This can be generalized to all three generations of leptons:

$$
m_{e}=g_{e} v \quad m_{\mu}=g_{\mu} v \quad m_{\tau}=g_{\tau} v
$$

\footnotetext{
${ }^{4}$ The abbreviation h.c. stands for Hermitian conjugate.
} 
The strength of the coupling is directly proportional to the lepton mass, hence the strongest probe of Higgs couplings to leptons is through the tau lepton. For this reason searches for the Higgs boson are rarely performed in $H \rightarrow \mu \mu$ or $H \rightarrow e e$ channels.

Quarks acquire mass in a similar manner, with the obvious addition of obeying $\mathrm{SU}(3)_{C}$ symmetry, the relevant Lagrangian terms are:

$$
\mathcal{L}_{\mathrm{Y}}=g_{u} \bar{Q}_{L} \phi u_{R}+g_{d} \bar{Q}_{L} \phi d_{R}+g_{u} \bar{u}_{R} \phi^{\dagger} Q_{L}+g_{d} \bar{d}_{R} \phi^{\dagger} Q_{R}
$$

where $g_{u}$ and $g_{d}$ are the Yukawa couplings to up and down quarks, $\bar{Q}_{L}$ is the left handed $\mathrm{SU}(2)_{L}$ quark doublet, and $u_{R}$ and $d_{R}$ are the corresponding right handed color-triplet weak-isospin singlets. As in the leptonic sector the mass terms are proportional to the Yukawa coupling:

$$
m_{u}=g_{u} v \quad m_{d}=g_{d} v \quad m_{s}=g_{s} v \quad m_{c}=g_{c} v \quad m_{b}=g_{b} v \quad m_{t}=g_{t} v
$$

Naturally the top quark is the best probe of Higgs couplings to quarks. Detailed measurements of this coupling can shed light on the nature of the Higgs.

Although the Higgs mechanism provides a reason for the broken symmetries resulting in particle masses, it does not predict what those masses should be. The reason for the strange and seemingly chaotic structure of fermion masses must lie in physics beyond the standard model.

\subsection{Higgs Boson Current Measurements}

The Higgs mechanism offers a tantalizing possibility to elegantly tie the SM together. To such an end the particle physics community has searched for the trademark sign of the mechanism, the Higgs boson, for roughly 40 years. Searches can be 
direct or indirect in nature; the most notable results from both types of searches are discussed below.

\subsubsection{Direct Searches}

The earliest direct searches were performed at LEP [10] using $2.5 \mathrm{fb}^{-1}$ of $e^{+} e^{-}$ collisions at roughly $200 \mathrm{GeV}$. The primary production and decay mode was associated production of a Higgs with a $Z$ followed by the $H$ decaying into $b \bar{b}$. Smaller contributions came from vector boson fusion and $H$ decays into $\tau \tau$ or $W W^{*}$. The final discriminants used were either the reconstructed Higgs mass, or a simple multivariable output based on $b$-tagging variables, likelihood functions and neural network outputs. At 95\% confidence level the SM Higgs was excluded below $114.4 \mathrm{GeV}$.

Concurrent with the analysis presented here, two general purpose LHC detectors began acquiring enough data to make direct exclusions. The first exclusions were at higher mass, above $m_{H}=150 \mathrm{GeV}$.

\subsubsection{Indirect Searches}

There are some theoretical restrictions on the Higgs mass based on precision measurements of the $W$ boson and $t$-quark masses. The radiative loop corrections for the two heaviest charged SM particles restrict the allowed Higgs mass. Figure 1.2 shows the available regions for a SM Higgs considering LHC/Tevatron/LEP direct exclusions (green bands), and the allowed region given the world average for the $t$ quark mass and the $W$ boson mass (red oval). The small overlapping region inside the red oval and within the green band represent the final hiding place for the SM Higgs boson. 


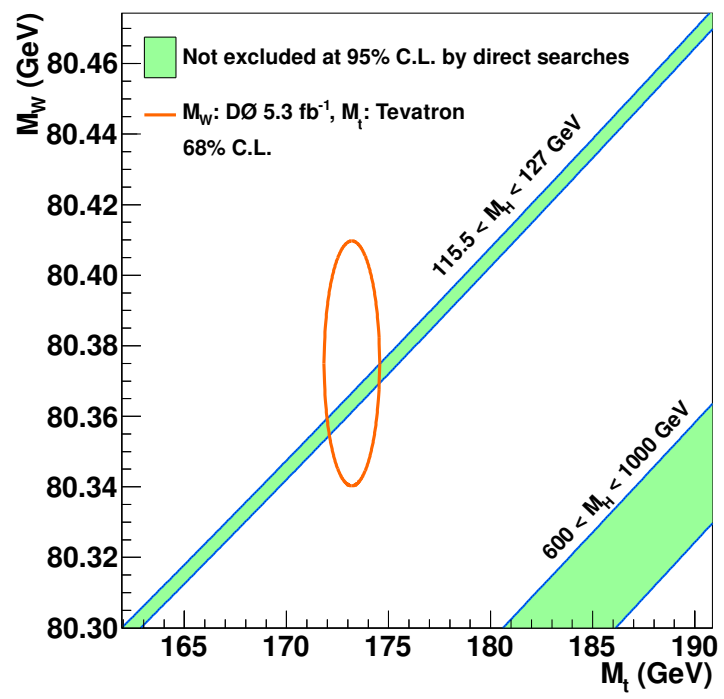

Figure 1.2. Restrictions of possible Higgs mass from $W$ boson and $t$-quark mass measurements. The overlap between the red oval and the green stripe represent the last allowed values for the SM Higgs.

\subsubsection{Theoretical Higgs Limits}

Purely theoretical evidence also restricts the possible Higgs mass [11]. As the energy scale $\Lambda$ increases, the allowed Higgs mass range is reduced. Figure 1.3 shows the allowed mass as a function of $\Lambda$. For large $m_{H}$, renormilization-group equations (RGEs) drive the Higgs self-coupling in a non-perturbative region that requires new physics in order for the Higgs self-coupling to not blow up (blue lines). If $m_{H}$ is small, the Higgs self-coupling goes negative creating an unstable electroweak minimum (yellow band). There are two additional metastable regions where the lifetime of the electroweak vacuum is longer than the age of the universe due to zero-temperature quantum fluctuations (red band) or thermal fluctuations (light blue band).

There of course exist theories beyond the standard model, the most prominent among them is Super Symmetry (SUSY), that deal with the problems contained in the SM. SUSY predicts enhanced coupling to down-type fermions while couplings to 


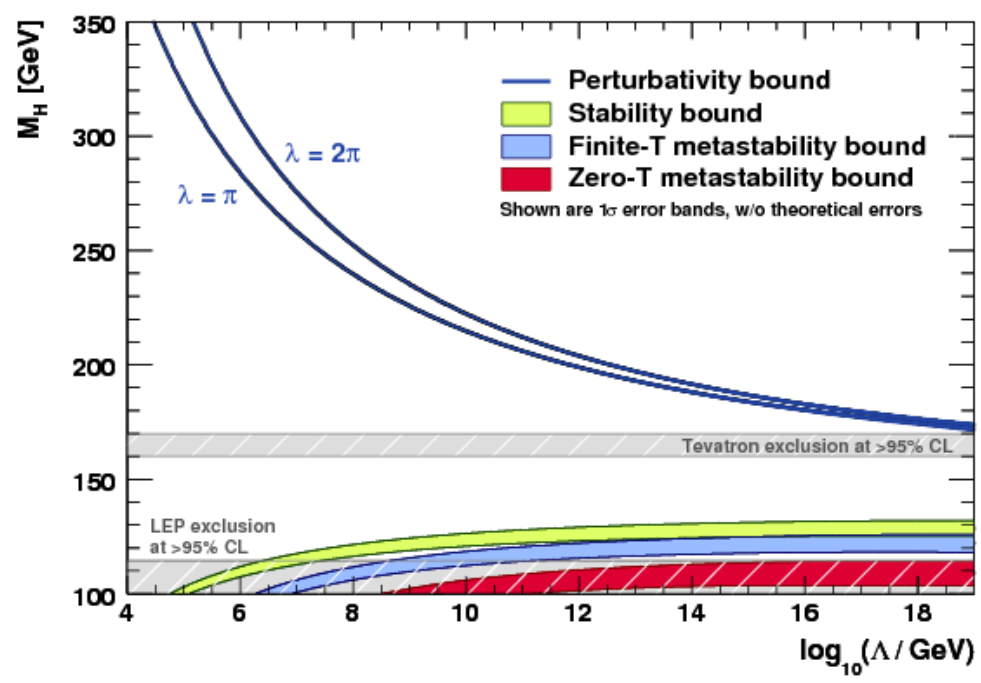

Figure 1.3. Theoretical restrictions for the Higgs mass plotted as a function of $\Lambda$, the energy scale.

up-type fermions are suppressed. Should they exist, these enhancements will likely be visible at the LHC.

\subsection{Higgs at the Tevatron}

Higgs searches at Fermilab are molded by the kinematics of the Tevatron. The various production mode cross sections are shown in Fig. 1.4. Gluon fusion (black line) is the dominant process with a cross section of roughly $1 \mathrm{pb}$ for any Higgs mass at $\sqrt{s}=1.96 \mathrm{TeV}$. In the low mass region, associated Higgs production (red and maroon dashed) with $W$ and $Z$ is the next-to-leading mode. Vector boson fusion (green line) is of the same order, but fractionally more important in the high mass region. Next-to-next-to-leading processes are several orders of magnitude less and contribute very little to the most dominant searches.

Searches are classified by final states which are derived from the particular Higgs decay mode, as plotted in Fig. 1.5. The dominant low mass decay is clearly $b \bar{b}$. Associated production provides the strongest probe for these decays; the main 


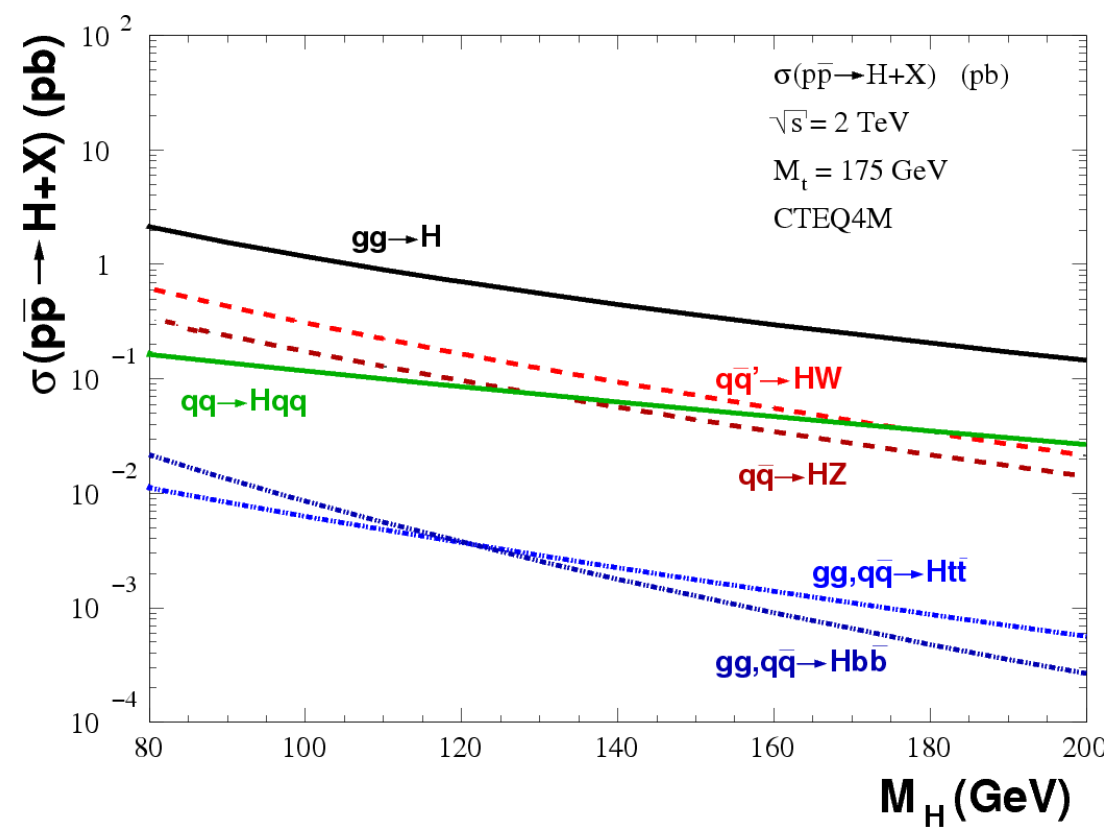

Figure 1.4. Higgs production mode cross sections at $\sqrt{s}=1.96 \mathrm{TeV}$ for $p \bar{p}$ collisions. Gluon fusion is the dominant process, however most searches rely most heavily on associated production to help improve background rejection.

contributions are from $W H \rightarrow \ell \nu b \bar{b}, Z H \rightarrow \ell \ell b \bar{b}, Z H \rightarrow \nu \nu b \bar{b}$. The sub-dominant process in the low mass region is $H \rightarrow \tau \tau$ and is favorable over $g g / c c$ final states since taus are generally easier to identify than gluon or $c$-quark jets. At high mass, vector boson fusion is the primary production mode with decays $H \rightarrow W W \rightarrow \ell \nu \ell \nu$. A search is also performed for $H \rightarrow \gamma \gamma$, which is about three orders of magnitude less than $H \rightarrow b \bar{b}$ but the background is very small making it a "golden" channel. Additional searches include the gluon fusion dominated tri-lepton searches $H \rightarrow$ $e e \mu / \mu \mu e / \tau \tau \mu$. No searches are performed at $\mathrm{D} \varnothing$ in the $H \rightarrow 4 \ell$ channel because the yields are too low. The best overall sensitivity is achieved when all channels are combined. 


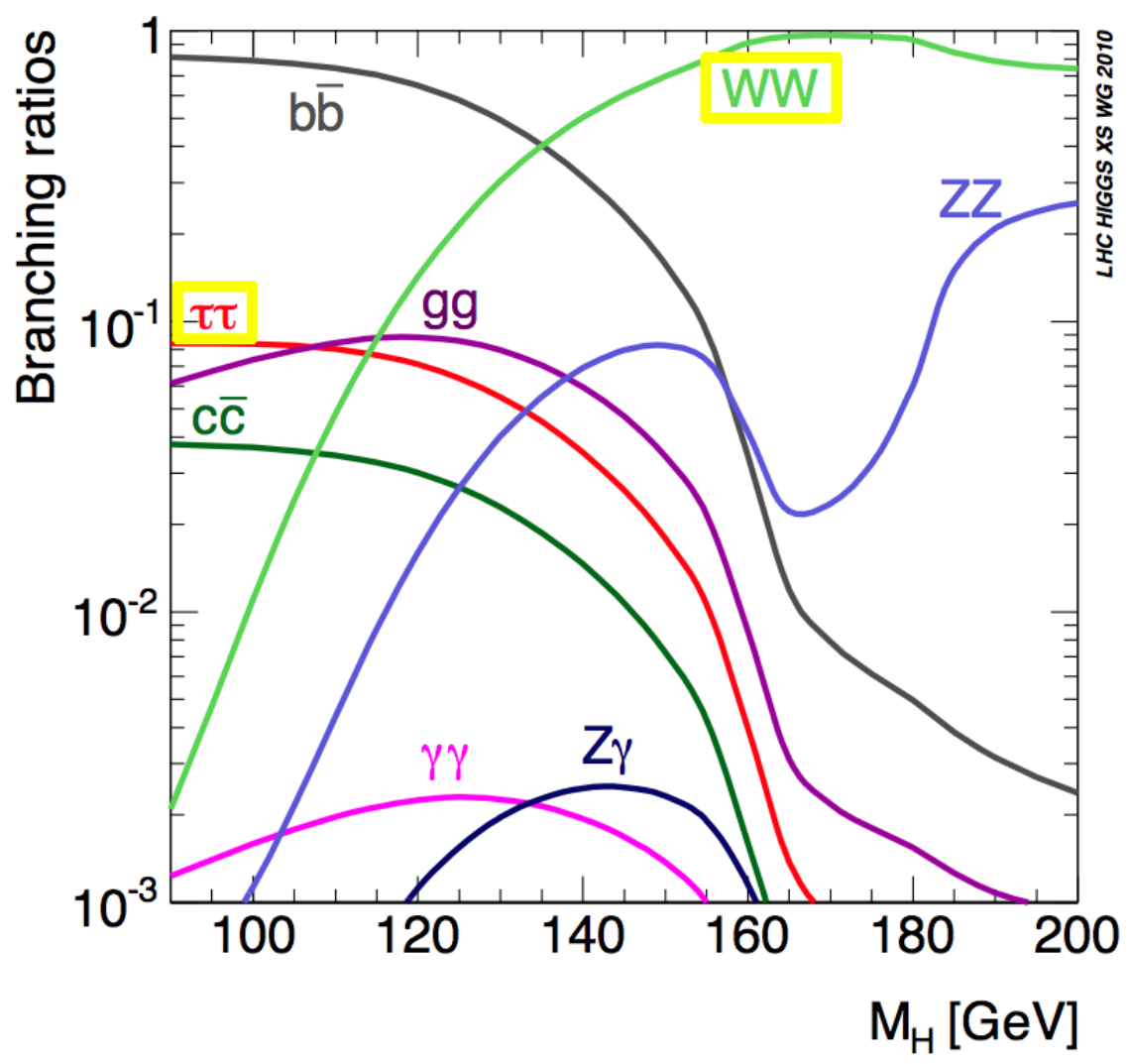

Figure 1.5. Higgs branching ratios. The primary decays considered at the Tevatron are $H \rightarrow b \bar{b}, W W, \tau \tau$ and $\gamma \gamma$. The $c \bar{c}$ and $g g$ modes are overrun by jet backgrounds, and the $Z Z$ mode does not have enough statistics to contribute.

\subsubsection{Why $H \rightarrow \tau \tau$ ?}

This analysis looks primarily for Higgs decaying into two tau leptons. Several factors played a role in the decision to pursue a search in this final state. The first is theoretical, since Higgs couplings to fermions are directly proportional to mass, that tau is an obvious choice as the third heaviest fermion. Furthermore, as seen in Fig. 1.5, the decay of the Higgs into $\tau \tau$ final states is the subdominant process in the low mass region and of the same order of magnitude as $c \bar{c}$ and $g g$. These two states, however, are nearly impossible to handle at hadronic colliders. The $b$-tagging process 
can clearly identify $b$-jets whereas $c$-jets and gluon jets are virtually indistinguishable, and swamped with other hadronic background processes.

The more practical reason for choosing this channel is that the primary channels of $H \rightarrow b \bar{b}$ and $H \rightarrow W W$ with all of their various productions and decay modes were all ready manned. An opening in the Tau ID group led seamlessly into a search in the $H \rightarrow \tau \tau$ channel.

Within the $H \rightarrow \tau \tau$ channel there are six possible final states to search for: $\tau_{h} \tau_{h}$, $\tau_{h} \mu, \tau_{h} e, e e, \mu \mu$, and $e \mu$ (where $\tau_{h}$ represents a hadronic tau decay). The percentage of each of these final states is shown in Fig. 1.6. Clearly the most probably final state from taus is $\tau_{h} \tau_{h}$, however, as will be shown later, hadronic tau decays are difficult to work with. Having one leptonic decay affords good rejection of background but higher branching fraction than two leptonic decays, hence searches are performed in the $\tau_{h} \mu$ and the $\tau_{h} e$ channels.

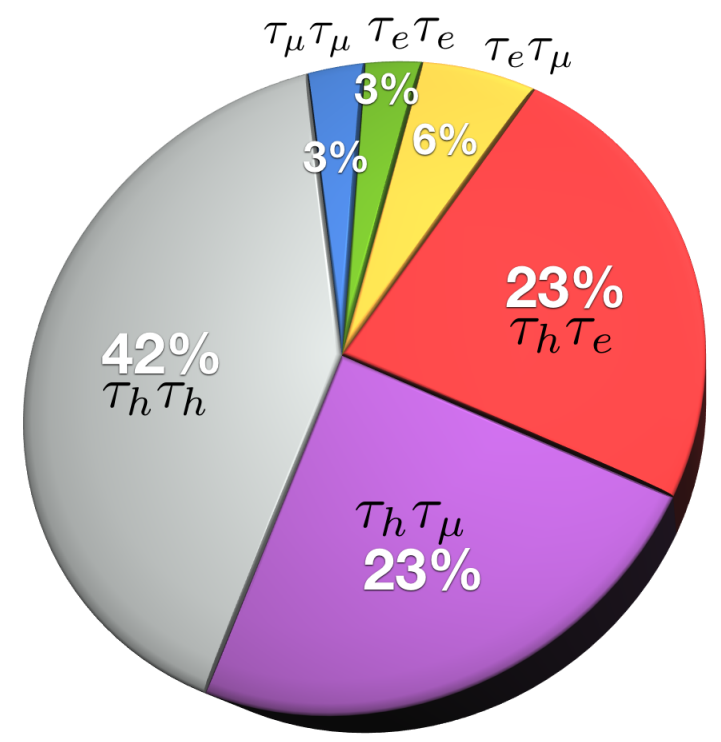

Figure 1.6. Branching fractions for $\tau \tau \rightarrow \tau_{x} \tau_{x}$. The most probable decay is for both taus to decay hadronically. With equal probability one will decay hadronically and the other leptonically. The three lepton only decays contribute only $9 \%$ combined. 
Of these two channels $\tau_{h} \mu$ is preferable because muon identification is in general easier than electron identification. In other words, many objects fake electrons, but very few objects fake muons. Additionally, the primary background to the searches are $Z \rightarrow \mu \mu$ and $Z \rightarrow e e$ respectively. For the same reason, it is easier to handle the $Z \rightarrow \mu \mu$ backgrounds.

The Stony Brook University group at D $\varnothing$ began analyses in both channels and this analysis builds on the thesis work of Katy Tschann-Grimm who graduated from SBU in 2011. Many new features were added and several crucial improvements were made to this search greatly improving its overall sensitivity. The $\tau_{h} \mu$ and $\tau_{h} e$ analyses are conducted in parallel using many of the same techniques. To achieve the best results the final limits are combined.

\subsection{2 $H \rightarrow \tau \tau$ Production and Decay modes}

This analysis searches for the Higgs boson in final states containing one electron, $e$, one hadronically decaying tau lepton, $\tau_{h}$, and at least two jets, $j j$ where the jets can be either quark jets, or gluon jets. The five process that give rise to this final state can be found in Tab. 1.4. In the first four processes the search is for decays of $H \rightarrow \tau^{+} \tau^{-}$and $H \rightarrow W^{+} W^{-}$. The final state electron can arise from either $\tau \rightarrow e \bar{\nu}_{e} \nu_{\tau}, W \rightarrow e \bar{\nu}_{e}$, or $W \rightarrow \tau \bar{\nu}_{\tau} \rightarrow e \bar{\nu}_{e} \nu_{\tau}$. The final state tau can be from prompt decay of the Higgs, or decay of the Higgs through a $W$ to a tau. To differentiate tau decays from $W$ decays the processes are labeled $\mathrm{VBF}_{\tau \tau}$ or $\mathrm{VBF}_{W W}$ etc.

There are three primary production modes: (1) associated production (Fig. 1.7 (a)) where two quarks produce a virtual ${ }^{5} Z$ or $W$, which decays into a real $Z$ or $W$ with a Higgs; the two jets come from the vector boson decay into hadrons. (2) Gluon

\footnotetext{
${ }^{5}$ Virtual particles may not have the appropriate mass, and can violate some laws as the exist only within the uncertainty principle.
} 
Table 1.4. Production and decay mode summary.

\begin{tabular}{c|c|c}
\hline \hline Process & Name & Denoted \\
\hline$q \bar{q} \rightarrow Z(\rightarrow q \bar{q}) H$ & Associated Production & ZH \\
$q \bar{q} \rightarrow W(\rightarrow q \bar{q}) H$ & Associated Production & WH \\
$g g \rightarrow H+(\geq) 2$ jets & Gluon Gluon Fusion & GGF \\
$q \bar{q}^{\prime} \rightarrow q \bar{q}^{\prime} H$ & Vector Boson Fusion & VBF \\
$q \bar{q} \rightarrow H(\rightarrow b \bar{b}) Z(\rightarrow \tau \tau)$ & Associated Production & $\mathrm{HZ}$ \\
\hline \hline
\end{tabular}

fusion (Fig. 1.7 (b)) where two gluons interact via a top loop and produce a Higgs; the two jets come from radiated gluons. (3) Vector boson fusion (Fig. 1.7 (c)) where two quarks radiate virtual vector bosons which collide and produce a Higgs; the two jets come from the original quarks which radiated the bosons. In all cases the $H$ will decay into $\tau^{+} \tau^{-}$or $W^{+} W^{-}$.

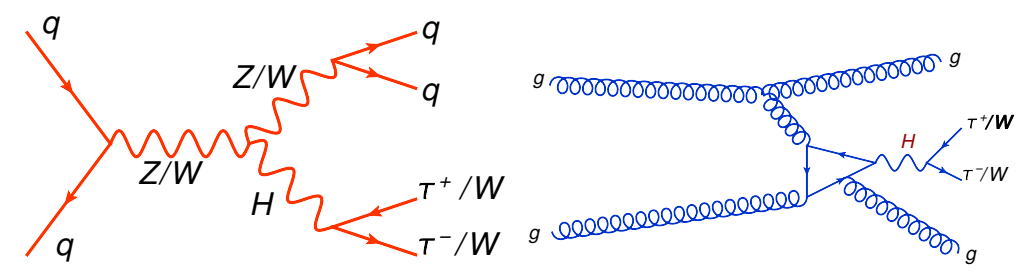

(a)

(b)

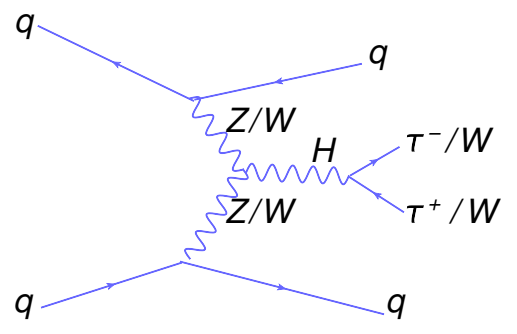

(c)

Figure 1.7. Feynman diagrams for the three production modes considered in this analysis: (a) associated production, (b) gluon fusion, (c) vector boson fusion. In any production mode the Higgs can decay into a pair of taus or a pair of $W$ 's. 
In the final line of Table 1.4, there is another version of associated production, where a virtual $Z$ produces a real $Z$ and a Higgs. The Higgs decays into $b$-jets and the $Z$ decays leptonically into two taus, which subsequently decay into one $e$ and one $\tau_{h}$. This process contributes only about $5 \%$ to the overall expected signal yield.

To gauge the importance of each of these production modes Fig. 1.8 shows the fractional expected signal yield as a function of Higgs mass over the considered range $105 \leq m_{H} \leq 150 \mathrm{GeV}$ for each production process and for $\tau \tau$ decays (solid lines) and $W W$ decays (dashed lines). While Fig. 1.5 shows simply the theoretical branching ratio, Fig. 1.8 convolves the production modes, decay ratios and final object selections (Section 4.4). The effective result is that the $\tau \tau$ decays are dominant in this analysis for $m_{H}<135 \mathrm{GeV}$.

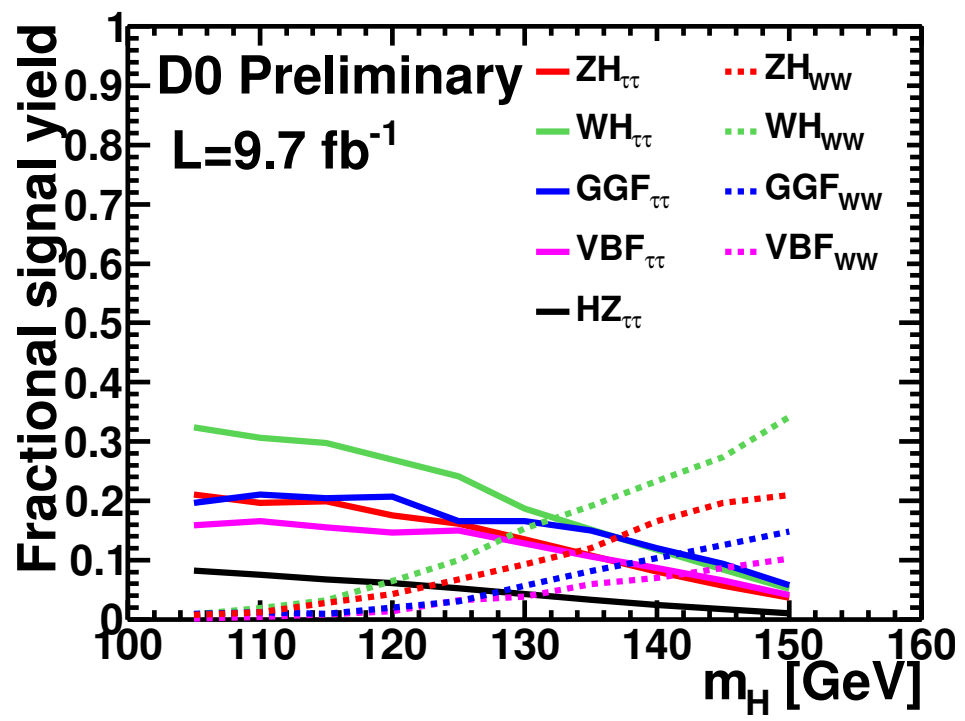

Figure 1.8. Fractional expected signal yields in the final selection. $\tau \tau$ decays are shown in solid lines and $W W$ decays in dashed lines. The contamination of $W W$ signals in the low mass region is less than the contamination from $\tau \tau$ signals in the high mass region. 
The decay products of the Higgs are crucial to identifying a potential candidate. Constructing the invariant mass of either the $\tau \tau$ or the $W W$ system will help identify the parent particle. If the Higgs has any mass less than $160.47 \mathrm{GeV}$ (two times the $W$ mass) in the case of the $W W$ decay one of the $W$ 's will be off-shell. In the case of $\tau \tau$ decays any Higgs mass is sufficient to produce two on-shell taus, however with any hadronic $\tau$ decay there are one or more neutrinos present (as discussed in Section 1.2) which make measurements of the invariant mass difficult.

Each process in Table 1.4 has unique kinematic properties that can help identify potential signal events. For instance, the two jets produced in VBF have very large $|\eta|$ values, hence tagging forward jets would increase the signal significance $\mathcal{S}_{\text {sig }}=S / \sqrt{B}$, where $S$ is the number of signal events, and $B$ is the number of background events. However, due to the very small statistics it is impractical to divide this analysis into searches for individual production processes. As demonstrated in Chapter 5 there are techniques to improve sensitivity beyond simple cut based methods. ${ }^{6}$

\subsection{Overview}

Chapter 2 discusses the Fermilab Accelerator complex and D $\varnothing$ detector. Chapter 3 introduces the algorithms used in identifying the particles needed in this search and Chapter 4 enumerates the requirements placed on these objects. Chapter 5 details the innovative multivariate techniques developed for this search. The main results including cross section limit ratio plots are found in Chapter 6. Chapter 7 shows how this analysis is combined with other $\mathrm{D} \varnothing$ and Tevatron searches. Finally it also includes a summary of the results presented here. The Epilogue provides an overview of the 2012 LHC Higgs search results.

\footnotetext{
${ }^{6}$ The LHC $H \rightarrow \tau \tau$ analyses divide their searches by production mode and are discussed in [12] and [13].
} 


\section{CHAPTER 2}

\section{EXPERIMENTAL SETUP}

The research discussed in the following chapters was conducted at the Fermilab National Accelerator Laboratory (FNAL) in Batavia, IL [14]. Approved by President Johnson in 1967, Fermilab's first major discovery came in 1977 with the discovery of the bottom quark [15]. After over twenty years, two more discoveries solidified Fermilab's place in history: the 1995 discovery of the top quark ([16], [17]) and the 2000 discovery of the tau neutrino [18].

\subsection{The FermiLab Accelerator Complex}

The crown jewel of Fermilab for the past 30 years has been the Tevatron. At center of mass energy $\sqrt{s}=1.96 \mathrm{TeV}$, it was the worlds highest energy particle accelerator until the LHC turned on in 2008. The Tevatron brings protons and antiprotons up to their ultimate energy as part of a chain of accelerators. The main components of the accelerator complex (Fig. 2.1, [19]) are the following:

Proton Source (0-750 $\mathbf{k e V})$ The first stage of the acceleration process is the Cockcroft-Walton generator, a voltage multiplier. Hydrogen gas is ionized, creating $\mathrm{H}^{-}$consisting of two electrons and a proton. The negative ions are accelerated over a potential difference of $750 \mathrm{keV}$.

Linac (750 keV - $400 \mathrm{MeV}$ ) The second stage is a linear accelerator, roughly 500 ft long. The $\mathrm{H}^{-}$ions ride oscillating electric fields and reach $400 \mathrm{MeV}$ before passing through a carbon filter that stops the electrons and only allows protons through.

Booster (400 MeV - 8 GeV) The third stage is the $468 \mathrm{~m}$ circular Booster ring. 


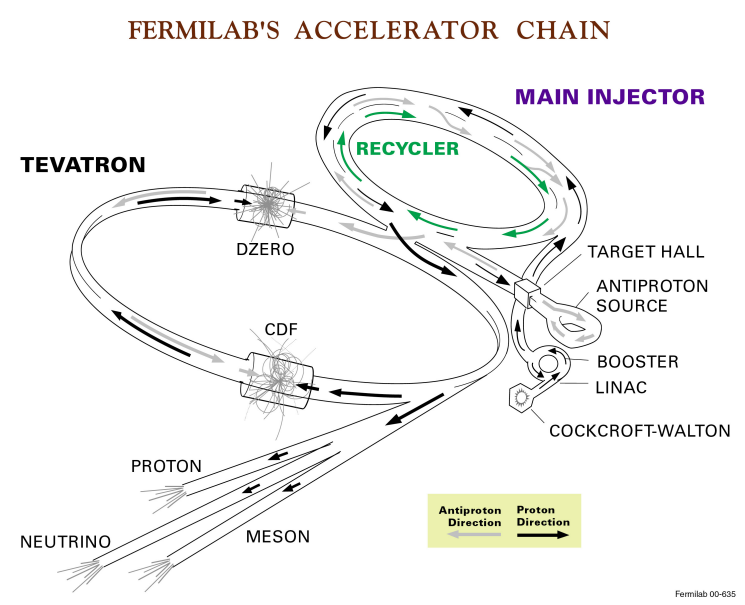

Figure 2.1. Schematic view of the Fermilab accelerator campus. The Tevatron has a radium of $1 \mathrm{~km}$, and houses both general purpose detectors: CDF and DØ.

Protons make roughly 20,000 revolutions achieving an energy of $8 \mathrm{GeV}$.

Main Injector $(8 \mathrm{GeV}$ - $150 \mathrm{GeV})$ The Main Injector is the newest accelerator in the chain and has been in operation since 1999 when it replaced the main ring, a series of magnets that shared the Tevatron tunnel. It is a 2 mile sheared oval tunnel that serves four major purposes: (1) it accelerates protons to $150 \mathrm{GeV}$, (2) it provides $120 \mathrm{GeV}$ protons that are used to create antiprotons, (3) it accelerates antiprotons received from the Antiproton Source to $150 \mathrm{GeV}$ and (4) it sends protons and antiprotons into the Tevatron.

Recycler and Antiproton Source To create antiprotons, $120 \mathrm{GeV}$ protons from the Main Injector are collided with a fixed nickel target, $p+N \rightarrow \bar{p}+h$, where $N$ is a nucleus, and $h$ stands for numerous hadrons (typically pions). Focusing magnets guide the antiprotons into the Accumulator ring; after a sufficiently high number of antiprotons are collected, they are sent into the Main Injector for acceleration and injection to the Tevatron.

Tevatron $(150 \mathrm{GeV}$ - $980 \mathrm{GeV})$ The final stage of the acceleration process is the Tevatron, a 4 mile circular accelerator that accelerates protons and antiprotons to 
nearly $1 \mathrm{TeV}$. The beams circulate in opposite directions within a single beam pipe, and are guided to collide at the center of two general purpose detectors, the Collider Detector Facility (CDF) and D.$^{1}$

\subsubsection{Accelerator Operations}

There are advantages and drawbacks to using antiprotons in a collider. At Tevatron energies, protons and antiprotons may circulate in opposite directions inside the same beam pipe and magnets. Creating and maintaining antiprotons is however, costly and challenging. Additionally, the bunch intensity of antiprotons is about a factor 10 less than that of protons and data is therefore acquired more slowly. From a physics stand point using $p \bar{p}$ collisions is preferred in making new resonances over $p p$ collisions since antiquarks are readily available inside an antiproton $(\bar{u} \bar{u} \bar{d})$ as valence quarks and thus produce $q+\bar{q}$ collisions with higher cross sections. At the LHC, for instance, antiquarks can only be found in the quark sea, and therefore have lower cross sections and contribute less energy to each collision.

Protons and antiprotons are injected into the Tevatron with the maximum number of particles available and with the maximum possible energy. After colliding particles for several hours the number of particles available for collisions becomes too low and running becomes inefficient. At this point new bunches of particles must be injected into the Tevatron, provided enough antiprotons have been stored in the Accumulator ring. Maintaining enough antiprotons to continually have collisions in the Tevatron is challenging, however for more than 20 years collisions were regularly produced and resulted in a steady stream of data to both experiments.

\footnotetext{
${ }^{1}$ The Tevatron is divided into sectors A-F with sections 0-4. The DØ detector is named for the location it occupies along the Tevatron.
} 
Operation of the Tevatron is broken into two main epochs: Run I (1987-1996) and Run II (2002-2011). Run I had collisions at $1.8 \mathrm{TeV}$ reaching a maximum luminosity of $\sim 2 \times 10^{31} \mathrm{~cm}^{-2} \mathrm{~s}^{-1}$. Upgrades to all accelerator components, and the addition of the Main Injector allowed for Run II to reach an energy of $1.96 \mathrm{TeV}$ with a maximum luminosity around $\sim 40 \times 10^{31} \mathrm{~cm}^{-2} \mathrm{~s}^{-1}$. During the nine years of Run II $11.9 \mathrm{fb}^{-1}$ of data were delivered to both the CDF and D $\varnothing$ experiments through thousands of "stores". Each store began to collide particles near the peak luminosity and collided until an instantaneous luminosity of $\sim 10 \times 10^{31} \mathrm{~cm}^{-2} \mathrm{~s}^{-1}$ was reached. In any one store several million events were recorded. Run II can be further divided into Run IIa and Run IIb. During a three month shutdown after producing $\sim 1 \mathrm{fb}^{-1}$ during Run IIa, some minor changes were made to both detectors.

\subsection{The D $\varnothing$ Detector}

The Run II D $\varnothing$ detector, shown in Fig. 2.2, is one of the two general purpose detectors situated around the Tevatron. It is roughly $30 \times 30$ x $50 \mathrm{ft}$ and weights 5,000 tons. It contains three main subsystems: tracking, calorimetry, and muon detectors. These systems are described briefly below and a more complete description can be found in $[20]$.

As in any general purpose detector $\mathrm{D} \varnothing$ uses the coordinate system $(r, \phi, \eta)$, where $r$ is the radial distance measured from the interaction point, or primary vertex (PV), $\phi$ is the azimuthal angle with range $(0,2 \pi)$, and $\eta$ (called pseudorapidity) is related to the polar angle by Eq. 2.1 and ranges $(-\infty, \infty)$. The standard polar angle is not Lorentz invariant, whereas $\eta$ is and allows for common angle measurements among different reference frames. 


$$
\eta=-\ln \left[\tan \left(\frac{\theta}{2}\right)\right]
$$

A standard Cartesian coordinate system $(x, y, z)$ is also considered, where $x$ points towards the center of the ring, $y$ points vertically upwards, and $z$ points along the beam line. Naturally, the point $(0,0,0)$ in both systems is the ideal interaction point, and the center of the detector. Since the initial longitudinal components of each collision are unknown it is more precise to consider the conservation of momentum only in the transverse $(x-y$ or $r-\phi)$ plane where initial the momentum is 0 . These quantities are denoted with a subscript $T$, i.e. transverse momentum, $p_{T}$, transverse mass, $m_{T}$ and missing transverse energy $\mathbb{E}_{T}$.

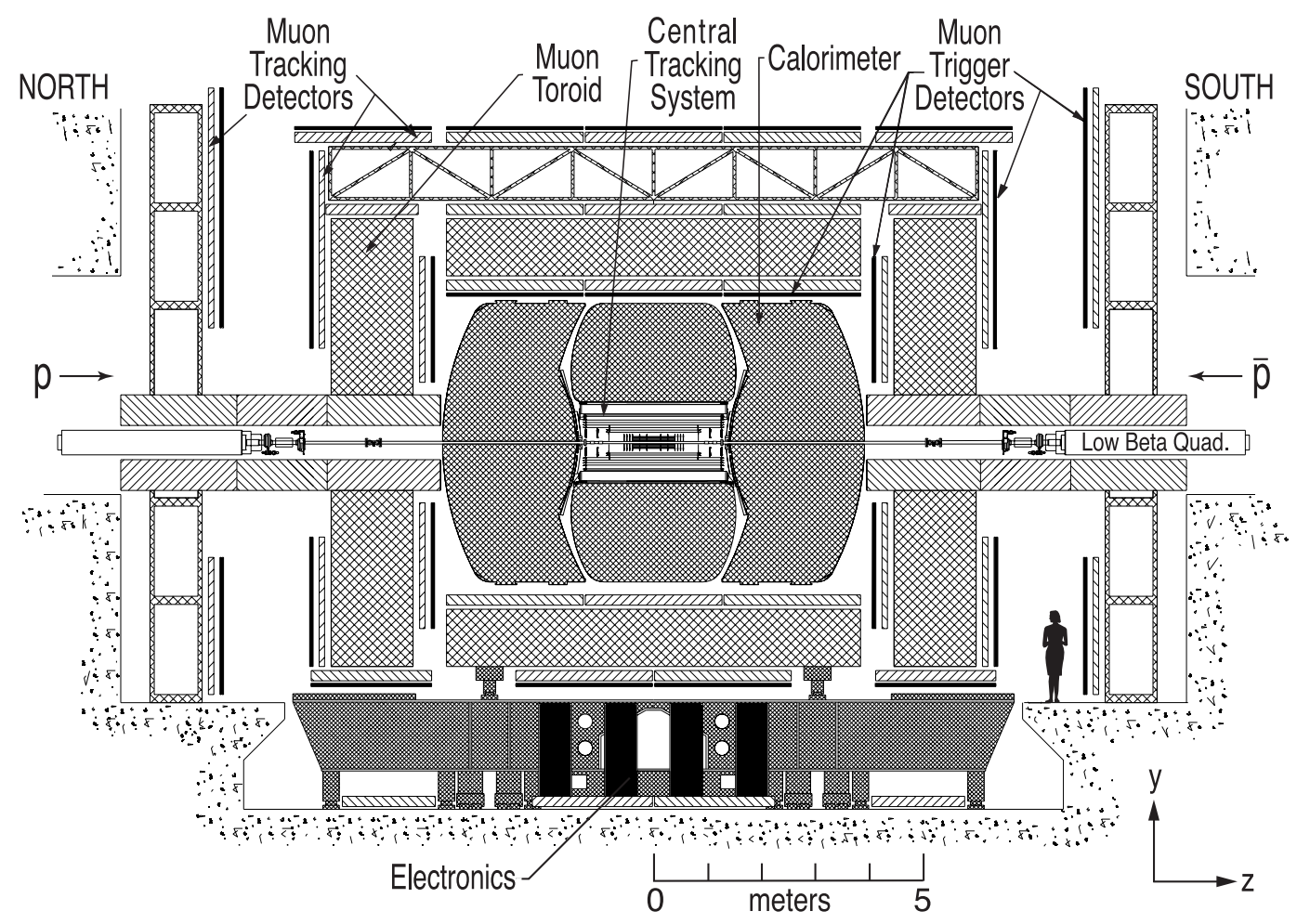

Figure 2.2. Schematic side view of the D $\varnothing$ detector showing (starting closest to the beam) the tracker, calorimeter and muon system. 


\subsubsection{Tracking}

The inner most layer of $\mathrm{D} \varnothing$ is the three part tracking detector. The silicon microstrip tracker (SMT) [21], and the central fiber tracker (CFT) [22], are contained within a $2 \mathrm{~T}$ solenoidal magnet. Together they can locate the PV to within $35 \mu \mathrm{m}$, over the interaction region of $\sim 25 \mathrm{~cm}$. A schematic view of this system is shown in Fig. 2.3. The third subsystem is the preshower detectors designed to enhance electron and photon identification as well as background rejection during triggering and offline reconstruction.

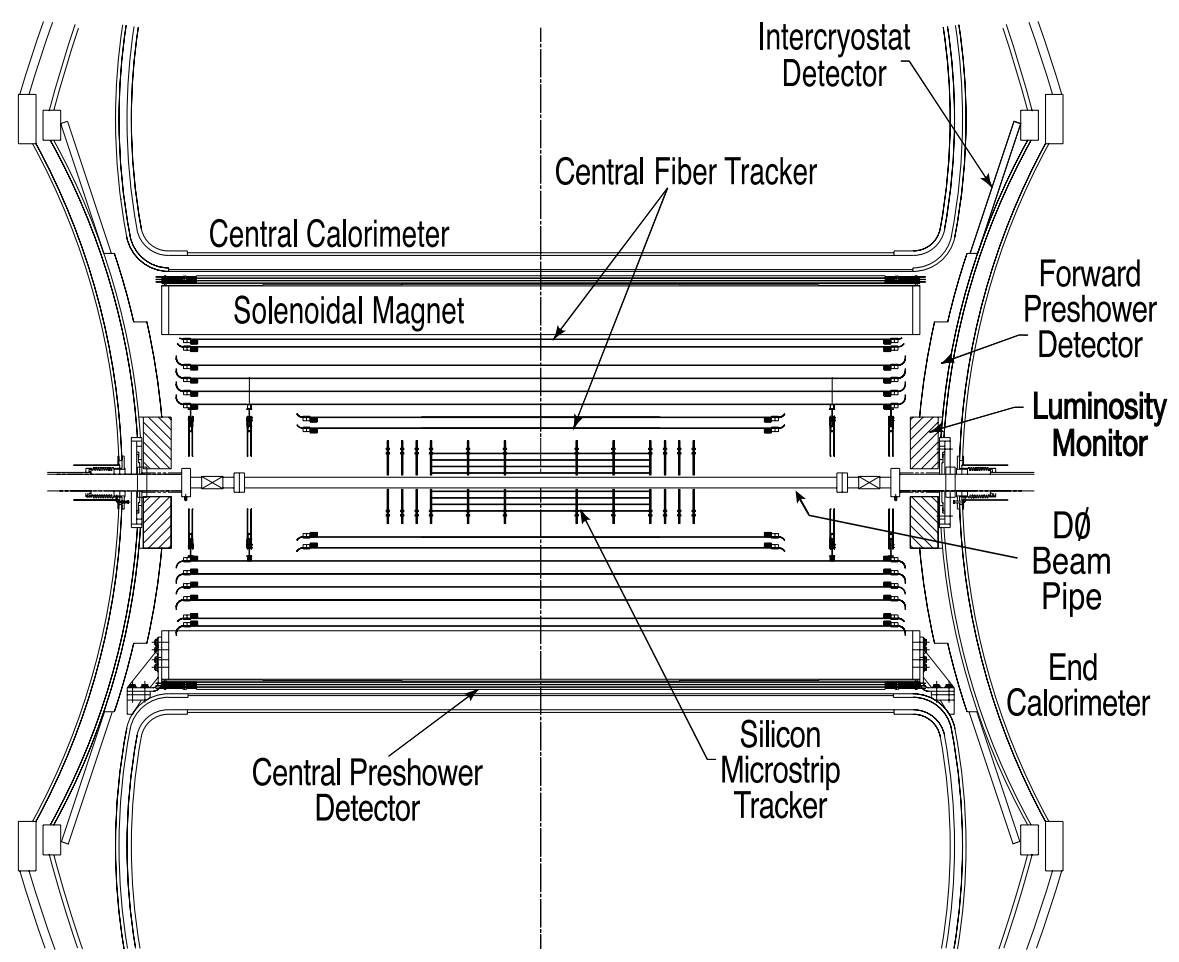

Figure 2.3. Schematic view of the inner detector designed to measure charged particles tracks and momenta. 


\subsubsection{SMT}

The SMT is designed to have active components perpendicular to the beam along the entire interaction length. Hence, it is divided into six barrels in the central region with centers $12.8 \mathrm{~cm}$ apart (Fig. 2.4). Each barrel has four readout layers that are rotated relative to each other to maximize the active area. Before the start of Run IIb a highly radiation hard inner layer called Layer 0 was added to minimize the loss of performance and resolution to the inner tracker due to radiation damage caused by the increased luminosity. At high $|z|$ each barrel is capped with an "F-disk," a double-sided 12 panel wheel in the $r-\phi$ plane. To cover the highest $|\eta|$ regions there are two additional units called "H-disks" which have 24 double-sided readout panels. Figure 2.5 shows the overlapping layers of a barrel module, as well as three panels of an H-disk.

Each silicon wafer is approximately $300 \mu \mathrm{m}$ thick, and made of strips of $p-n$ junction diodes that are operated with reverse bias. The electric field of the diode accelerates the charges created from the ionization towards the electrode. A SVXIIe chip uses analog to digital converters (ADCs) to create a signal that is read out by the high density interconnects (HDIs). The SMT reads out nearly 800,000 total channels.

Silicon detectors have much better resolution than previous tracking methods (wire/cloud chambers) but must be operated in extremely low noise environments which require significant cooling. Dissipating the heat generated by the SVXIIe chips, while minimizing dead areas poses a significant engineering problem. A coolant of water $/ 30 \%$ ethylene glycol mixture is supplied at $-10{ }^{\circ} \mathrm{C}$ and runs through the support structure of the SMT. 


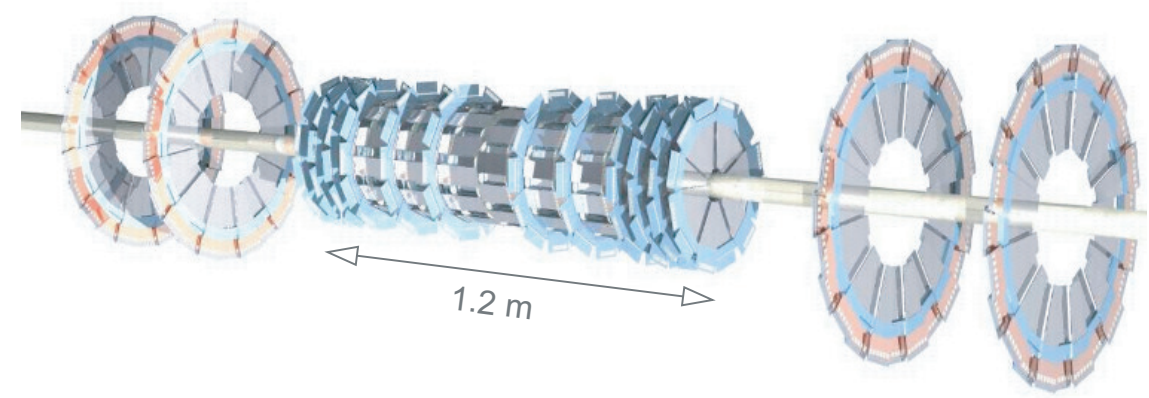

Figure 2.4. Schematic view of the inner silicon tracking system showing six barrels and 14 disks. The majority of the SMT is designed to be perpendicular to the beam direction.

\subsubsection{CFT}

The larger of the two tracking units is the CFT. Encapsulating the silicon detector but still contained within the solenoid, it is the principle instrument for measuring particle track momenta, and since it processes signals faster than the SMT, it provides the first tracking information to the trigger.

The CFT is made up of eight concentric rings housing $835 \mu \mathrm{m}$ diameter scintillating fibers. To allow room for the SMT H-disks, the inner two cylinders are only $1.66 \mathrm{~m}$ long, and the outer six cylinders are all $2.52 \mathrm{~m}$, and hence provide tracking information up to $|\eta| \lesssim 1.7$. Each cylinder has two doublets of fibers, one oriented along the $z$ direction, and the second at $+3^{\circ}(u)$ or $-3^{\circ}(v)$. From the center there are four doublet $(z u-z v)$ layers. This orientation provides the most efficient tracking measurements given the confined space. The inherent doublet resolution is $100 \mu \mathrm{m}$. The 76,800 fluorescent-doped fibers are mirrored on one end, and connected to clear fiber wave guides of the same diameter on the other end. The waveguides are between 7.8 - $11.9 \mathrm{~m}$ long and carry the light below the detector to visible light photon counter (VLPC) cassettes, which then convert light into an electric signal. The routing of these fibers can be seen in Fig. 2.6. 


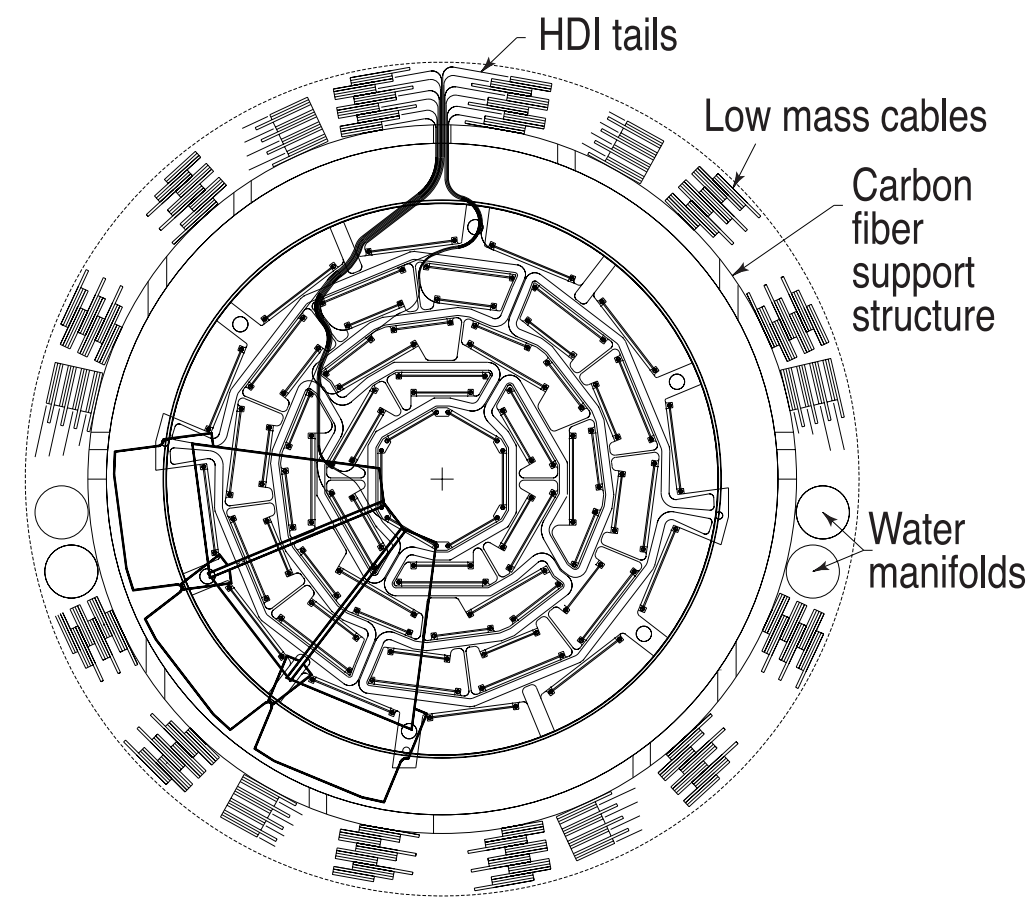

Figure 2.5. Schematic end view of the silicon tracking system with overlapping layers to maximize the number of hits per track.

\subsubsection{Solenoid}

The tracking solenoid magnet was installed for Run II and many of its specifications were dictated by limitations set by the existing $\mathrm{D} \varnothing$ detector. The main considerations were: (1) create as large and as uniform a field as possible over the volume available and (2) make it as thin as possible to allow more room for the tracker. The solenoid is $2.73 \mathrm{~m}$ long and $1.42 \mathrm{~m}$ in diameter, with a maximum field strength of $2 \mathrm{~T}$. The superconducting cables are made of Rutherford-type multifilamentary $\mathrm{Cu}: \mathrm{NbTi}$ and have a diameter of $0.848 \mathrm{~mm}$. The polarity of the magnet can be changed remotely which allows for the reduction of systematic errors caused by nonuniformities in the detector. With the field directed along $\pm z$, charged particles are bent through the tracking detector in the $r-\phi$ plane. In conjunction with the 


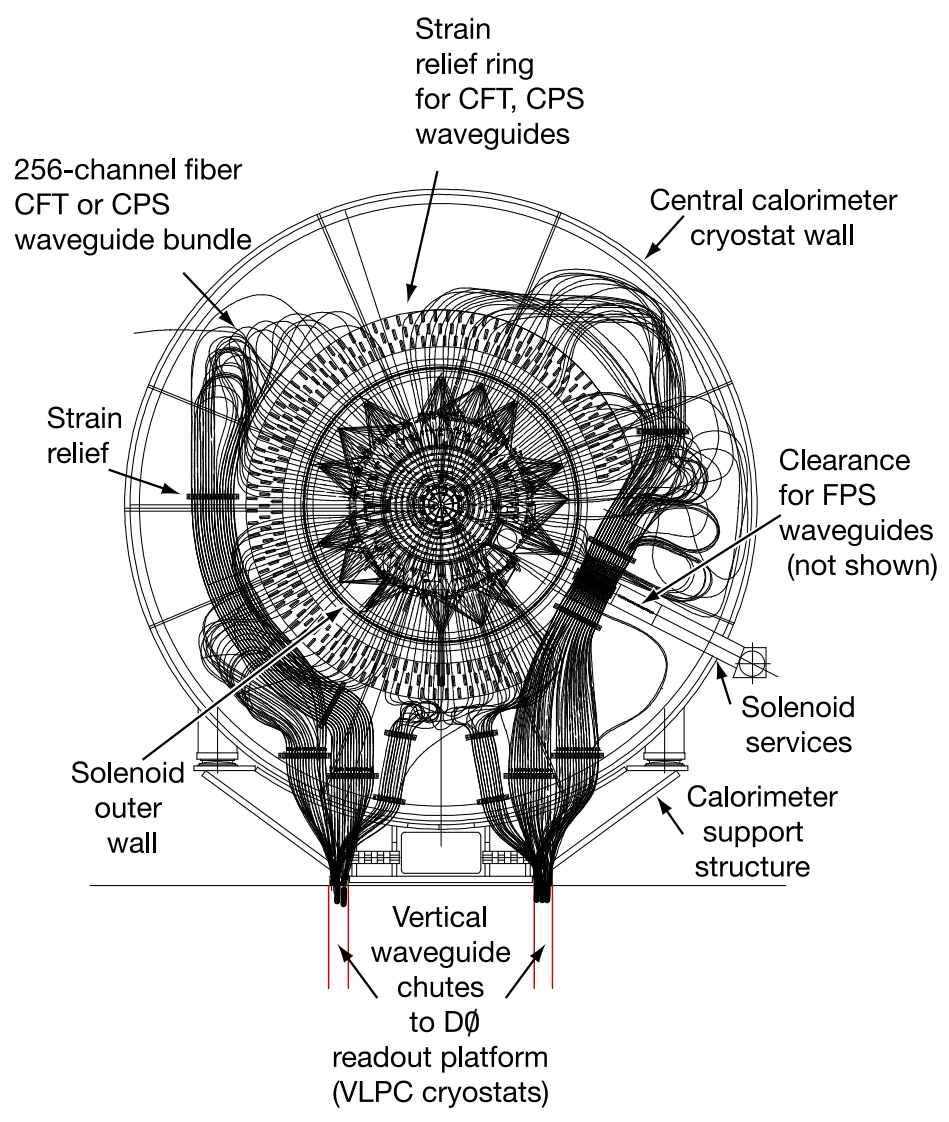

Figure 2.6. Schematic end view of the CFT and waveguides that guide the signals underneath the detector for processing.

toroidal magnet (Section 2.2.3) a nearly uniform magnetic field is produced throughout the detector (Fig. 2.7).

\subsubsection{Preshower}

Outside the solenoid are the central preshower (CPS) and forward preshower (FPS) detectors (Fig. 2.3). The CPS and FPS are designed to help identify electrons and photons as well as reject background. They function as both tracking detectors and calorimeters, helping to match central tracks to calorimeter showers and to correct (offline) central and end cap calorimeter measurements for losses due to the solenoid itself or other support materials. Strips of overlapping triangular scintillators make up 


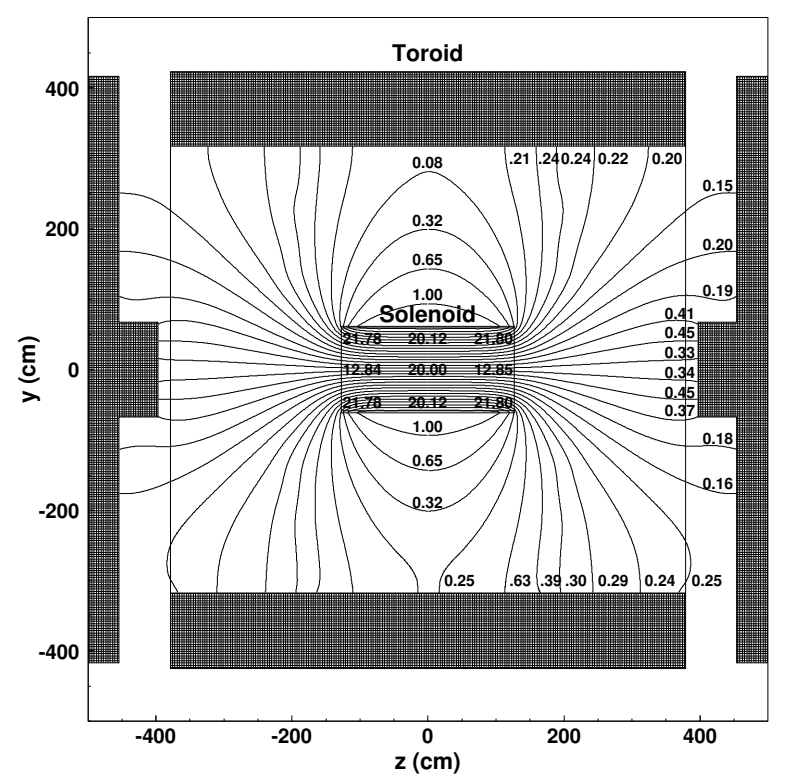

Figure 2.7. Approximate field lines due to the solenoid and toroid magnets.

both detectors which allow particles to pass through more than one strip enhancing the spacial resolution. Each scintillator is embedded with a wavelength-shifting fiber (WLS) and is coupled to the same type of clear fiber used in the CFT and are read out by the same VLPC cassettes. The CPS covers the region $|\eta|<1.3$ and has a radial thickness of only $2.4 \mathrm{~cm}$, while the FPS covers the region $1.5<|\eta|<2.5$ and has similar thickness in $z$.

\subsubsection{Calorimeter}

The $\mathrm{D} \varnothing$ calorimeter, shown in Fig. 2.8, is designed to measure the energy of electrons, photons, and jets, while also aiding the identification and tracking information for those particles. It is composed of three separate cryostat modules which house the active material, liquid argon, as well as the absorber material, primarily depleted uranium. These modules are referred to as the central calorimeter (CC), end calorimeter (EC). The CC covers regions $|\eta| \lesssim 1$, and the EC covers $0.7 \lesssim|\eta| \lesssim 4$. 


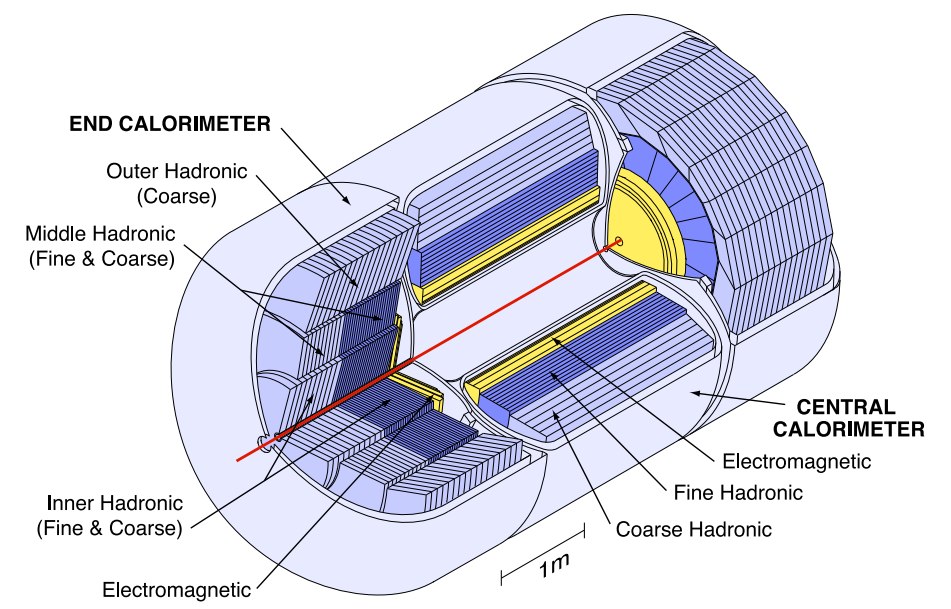

Figure 2.8. Schematic view of the calorimeter. It is more finely segmented in the electromagnetic portion closer to the beam to provide better resolution. The absorber material is mostly depleted uranium, and the active material is liquid argon.

Each cryostat has three main sections: an electromagnetic (EM), a fine hadronic, and a coarse hadronic each maintained at around $90 \mathrm{~K}$. The electromagnetic sections use nearly pure depleted uranium for the absorber material which is 3 (4) mm thick in the $\mathrm{CC}(\mathrm{EC})$. The absorber in the fine hadronic sections is made from $6 \mathrm{~mm}$ thick uranium-niobium (2\%). The coarse hadronic has $46.5 \mathrm{~mm}$ thick absorber plates made of copper in the CC, and stainless steel in the EC. A schematic view of a calorimeter cell is shown in Fig. 2.9. By grounding the absorber plate, and holding the signal boards at $\sim 2.0 \mathrm{kV}$ an electric field is created. When an incoming particle collides with the absorber plate it will produce electrons, positrons and photons, which in turn ionize the liquid argon. The free electrons are then accelerated across the 2.3 mm gap toward the signal board and collected. The amount of charge collected is proportional to the original particles energy.

The readout of calorimeter cells happens in layers seen in Fig. 2.10. The cells are offset from one another to ensure that particles pass through several active layers. The cells are grouped by depth into pseudo-projective "towers" of roughly equivalent 
$\eta$ and $\phi$ (cells boundaries are aligned perpendicular to the beam but towers point along constant $\eta$ lines). The transverse size of readout cells is comparable to the size of the expected shower: EM showers are roughly 1-2 cm, but hadronic calorimeter showers are about $10 \mathrm{~cm}$. Towers have sizes $\Delta \eta \approx 0.1$ and $\Delta \phi=2 \pi / 64 \approx 0.1$.

There are 55,296 calorimeter channels to be read out. Raw signals are first sent to preamplifiers located on the exterior of each cryostat, these signals are then sent to baseline subtraction (BLS) boards underneath the detector, which in turn are sent $130 \mathrm{~m}$ by analog drivers to ADCs where they enter the data acquisition system.

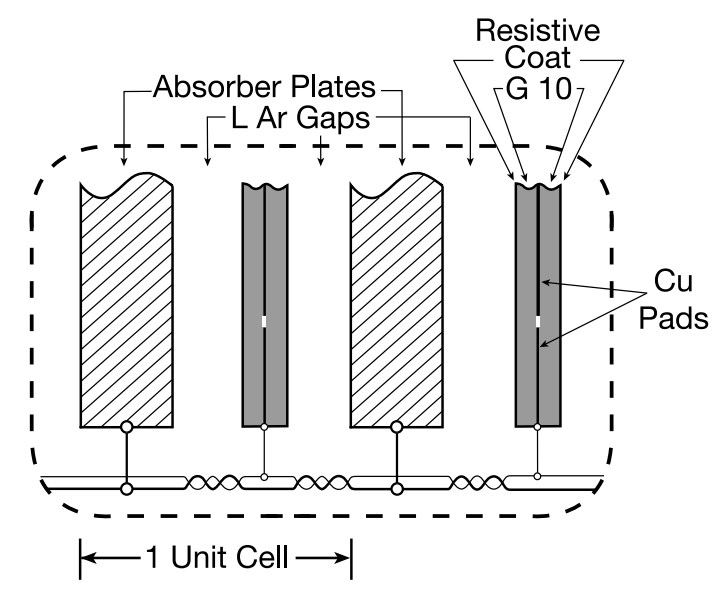

Figure 2.9. Schematic view of one calorimeter cell. Free electrons from the ionized argon gas are accelerated over a potential difference of $2 \mathrm{kV}$ and collected on the signal board.

\subsubsection{Muon System}

The outermost layer of the $\mathrm{D} \emptyset$ detector is the muon system, which is designed to measure the momenta and tracks of muons. Owing to their heavy mass $\left(200 m_{e}\right)$ muons with energy 3.5-4 GeV will penetrate all the absorber material in the calorimeter and enter the muon system, which has three components: (1) drift tubes for 


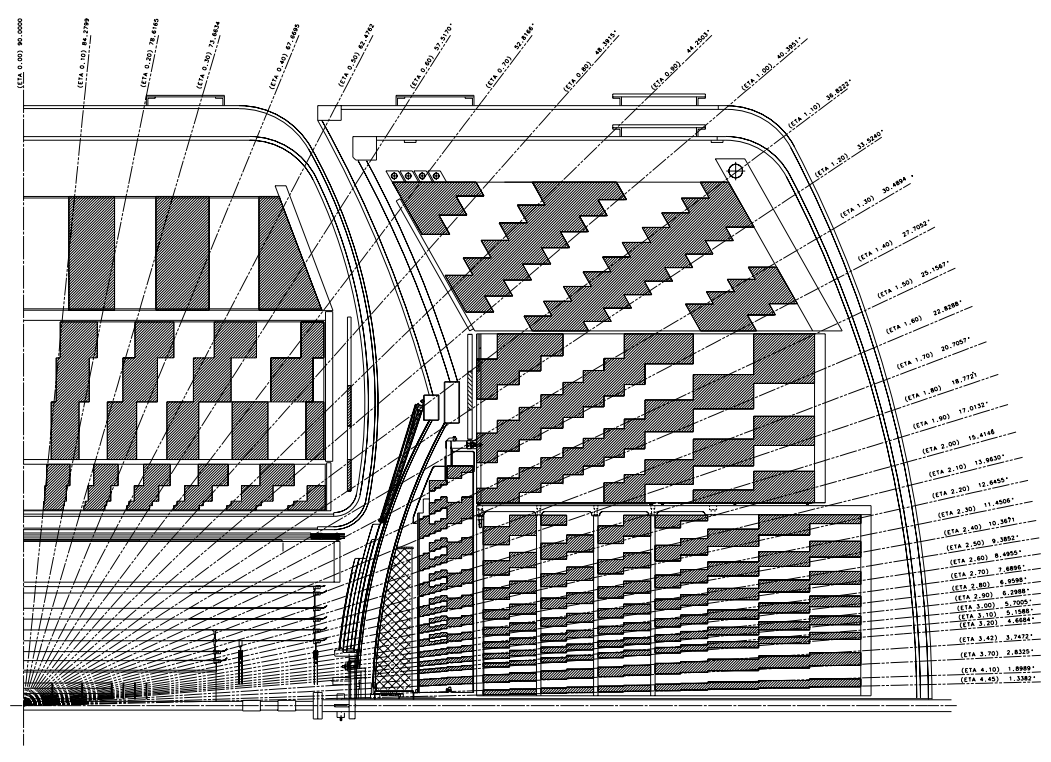

Figure 2.10. One quadrant of the calorimeter. The finer segmentation is located nearer the beam. There is only minimal EM calorimeter coverage in the region $1.1<\eta<1.3$.

tracking (momentum), (2) scintillators for triggering and (3) a toroidal magnet for helping measure momentum. A significant contribution of background muons from cosmic rays and other sources can be rejected by scintillators that enclose the entire detector.

The wide angle muon spectrometer (WAMUS) provides coverage up to $|\eta|<$ 1, and uses proportional drift tubes (PDTs). Figure 2.11 shows the three layered structure of the muon system. Moving radially outward a muon first strikes Layer A which is closest to the beam. It then enters the toroidal magnet $(1.8 \mathrm{~T})$ which will bend the muon in the $\pm z$ direction. Next the muon strikes the $\mathrm{B}$ and $\mathrm{C}$ layer detectors. There are no scintillators in the central B layer, and because of support systems for the calorimeter, there is no muon coverage in the region $225^{\circ}<\phi<310^{\circ}$. 
The forward angle muon spectrometer (FAMUS) has a similar structure, but utilizes mini drift tubes (MDTs) because of their better performance in the high rate of the forward environment and has coverage from $1.0<|\eta|<2$. There are scintillators on each layer of the forward muon system, and the north and south end toroids have a strength of $1.9 \mathrm{~T}$. All three toroidal magnets have their polarity regularly reversed independently from the central solenoid.

The PDTs and MDTs work very similarly to the liquid argon detector. As a muon passes through the gas mixture of $84 \%$ argon, $8 \%$ methane, and $8 \% \mathrm{CF}_{4}\left(\mathrm{CF}_{4^{-}}\right.$ $\mathrm{CH}_{4} 90 \%-10 \%$ ) in the WAMUS (FAMUS) it will ionize the gas. The time it takes the charge to drift to the anode wire gives a measurement of the muons position. Muon scintillators are roughly $26 \mathrm{~mm} \times 15 \mathrm{~mm}$, and contain WLS bars that carry light to a $25 \mathrm{~mm}$ photomultiplier tube.

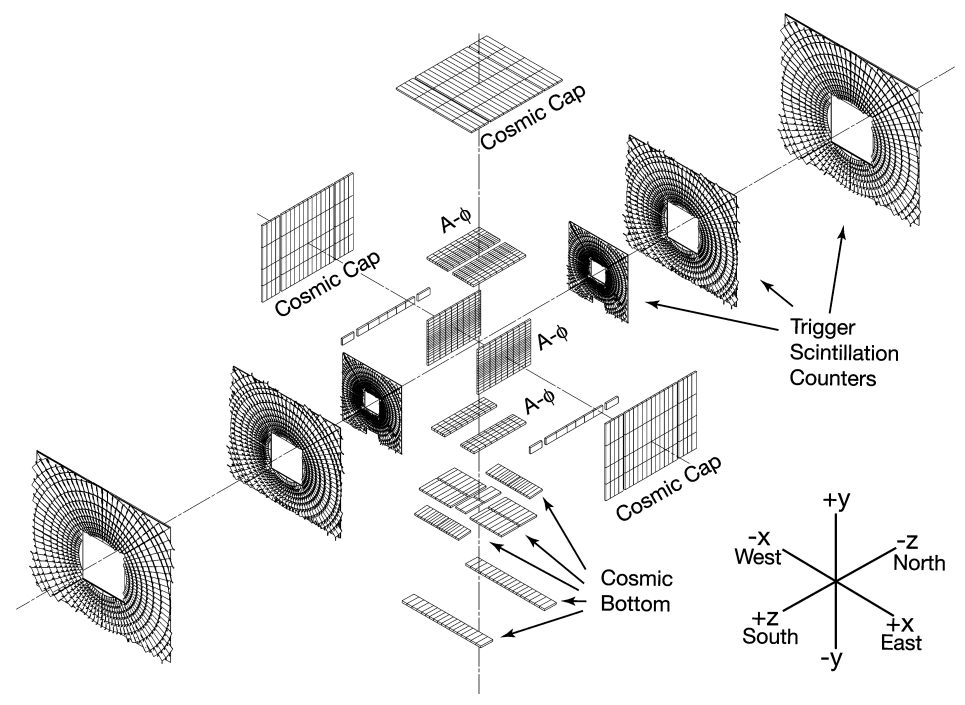

Figure 2.11. Schematic exploded view of the three layered muon system. The toroidal magnet is not pictured. 


\subsubsection{Luminosity Monitors}

The luminosity monitors (LM) are designed to measure the Tevatron luminosity at the $\mathrm{D} \varnothing$ interaction point. It can also measure beam halo rates and make a crude measurement of the $z$ position of the PV. The LM consist of two sets of $2415 \mathrm{~cm}$ long scintillating wedges that surround the beam pipe. The schematic drawing in Fig. 2.12 shows that they cover $2.7<|\eta|<4.4$, are located at $|z|=140 \mathrm{~cm}$.

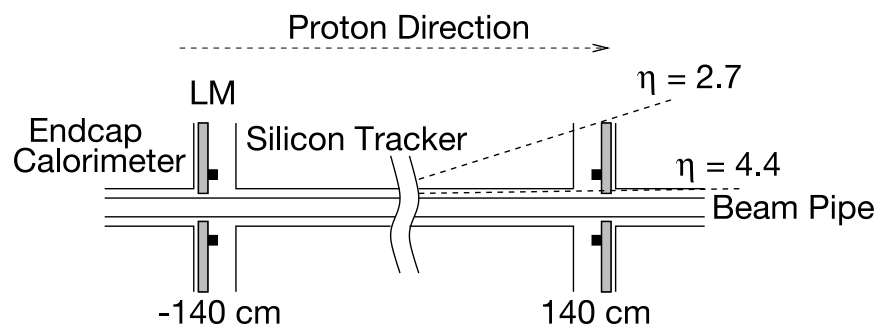

Figure 2.12. Schematic drawing showing the position of the luminosity monitors \pm 140 $\mathrm{cm}$ up and down stream from the interaction point.

The LM count the average number of inelastic $p \bar{p}$ collisions per beam crossing $\left(\bar{N}_{L M}\right)$ to determine the luminosity $(\mathcal{L})$ according to Eq. 2.2, where $f$ is the beam crossing frequency, and $\sigma_{L M}$ is the effective cross section of $p \bar{p}$ collisions taking into account the LM acceptance and efficiency.

$$
\mathcal{L}=\frac{f \bar{N}_{L M}}{\sigma_{L M}}
$$

To distinguish actual inelastic events from beam halo events the LM makes time-of-flight measurements assuming that particle originates from a $p \bar{p}$ interaction. By taking the difference in the time of flight $z_{v}=\frac{c}{2}\left(t_{-}-t_{+}\right)$to be $\left|z_{v}\right|<100 \mathrm{~cm}$, the LM ensures the event is actually a $p \bar{p}$ collision from within $\mathrm{D} \varnothing$, as opposed to a beam halo interaction. 


\subsubsection{Trigger and Data Acquisition}

The vast majority of collision events at any hadron collider are high cross section so-called "minimum bias" ( $p \bar{p} \rightarrow+X)$ events. The cross section of inelastic collisions is $50 \mathrm{mb}$, many orders of magnitude greater than other processes studied at D $\varnothing$ $\left(\sigma_{W} \sim \mathrm{nb}, \sigma_{H} \sim \mathrm{pb}\right)$. To sort through the large number of collisions and select the events of particular interest, D $\varnothing$ uses a three level trigger system: Level 1 (L1) is purely hardware, Level 2 (L2) is a combination of hardware and microprocessors, and Level 3 (L3) is a farm of microprocessors.

The purpose of a three leveled trigger systems is to sequentially reduce rates at which events are accepted. Table 2.1 shows the trigger rates and latency of each level. Successive trigger levels are more and more sophisticated and only events that meet the increasingly stringent conditions are passed through the trigger chain.

Table 2.1. Summary of approximate trigger rates.

\begin{tabular}{c|c|c}
\hline \hline Level & Rate & Latency \\
\hline Collisions & $1.7 \mathrm{MHz}$ & - \\
L1 & $1.6 \mathrm{kHz}$ & $3.6 \mu \mathrm{s}$ \\
L2 & $800 \mathrm{~Hz}$ & $100 \mu \mathrm{s}$ \\
L3 & $50 \mathrm{~Hz}$ & $150 \mathrm{~ms}$ \\
\hline \hline
\end{tabular}

Figure 4.3 shows a schematic version of the trigger processes and how the trigger is clearly integrated with the data acquisition (DAQ) system.

Level 1 - L1 receives information for every event from the calorimeter, central tracking trigger and the muon trigger. These systems select interesting events with energy or track momentum above specific thresholds. Additionally there are combined thresholds of the tracking and muon triggers to identify muon tracks.

Level 2 - L2 looks for correlations in objects from L2 preprocessors from various 
detector subsystems. Events with correlated basic physics objects are stored in the L2 buffer while awaiting the L3 accept.

Level 3 - L3 is a programmable software trigger that makes decisions based on fully formed physics objects and their relationships such as $\Delta \eta, \Delta \phi$ or their invariant mass. Specific object information like jet cone size or electromagnetic fraction are used and can be combined or adjusted (prescaled) at the start of each run to control the accept rate. Events out of L3 are written to tape for storage.

DAQ - COOR is the online processor that interacts directly with the trigger framework and the DAQ supervising systems. It can send commands to any trigger level and maintains the database of trigger names and parameters.

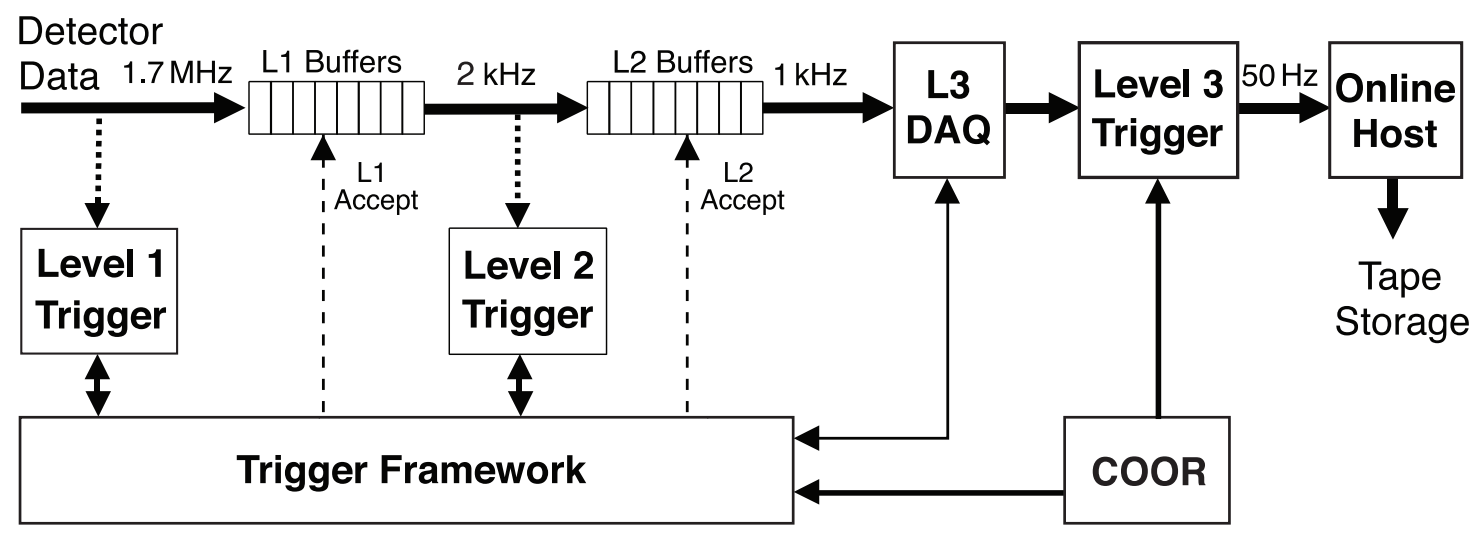

Figure 2.13. Schematic view of the trigger system shows each level, and it's integration into the data acquisition system.

\subsection{Monte Carlo Simulation}

An essential key to any particle physics experiment is the simulation of the physical processes considered. This typically involves modeling all processes that 
have the same detector signature as the primary process. Additionally modeling of the signal process can also be performed.

For the analysis presented here the $Z+$ jets, $W+$ jets and $t \bar{t}$ backgrounds were all simulated using ALPGEN as a parton level generator, with PYTHIA used for particle showering and hadronization [23]. The di-boson backgrounds were generated with PYTHIA version 6.319 and TAUOLA was used for all tau decays [24]. The simulated events then pass through a complete detector simulation in GEANT3, digitization (D0SIM), and reconstruction (D0RECO). Collectively this is known as Monte Carlo (MC) simulation.

$\mathrm{MC}$ simulations of each of the nine signal processes are created in $5 \mathrm{GeV}$ intervals from $105 \mathrm{GeV}$ to $150 \mathrm{GeV}$. CTEQ6L1, a library of parton distribution functions, is used to calculate next-to leading order (NLO) cross sections for all processes. For VBF next-to-next-to leading order (NNLO) is used. The program HDECAY is used to calculate SM Higgs branching fractions. The number of events, cross section, and MC request IDs are listed in the tables in Appendix B.

To account for various changes in both the Tevatron and DØ performance several versions of MC simulations were made corresponding to the different epochs of data taking. $\mathrm{MC} 2 \mathrm{a}, \mathrm{MC} 2 \mathrm{~b} 1, \mathrm{MC} 2 \mathrm{~b} 2$, and $\mathrm{MC} 2 \mathrm{~b} 3$ are all used, and normalized to the appropriate integrated luminosity for that epoch (MC2b3 is normalized to Run IIb3+4). In all of the MC epochs it is found that the data and MC do not perfectly agree, therefore a number of standard scale factors are applied to the MC to bring them into better agreement with data. The MC is first corrected for variations within a luminosity block to match zero bias data. ${ }^{2}$ Another correction factor, again derived from data, is added to account for changes in the longitudinal position of the primary vertex. The $p_{T}$ distributions of the $W$ and $Z$ are known to disagree with data, so an

\footnotetext{
${ }^{2}$ Data taken with no collisions.
} 
additional correction factor, which for the $Z$ is dependent on the number of jets, is applied to correct the shape of the $p_{T}$ distributions. 


\section{CHAPTER 3}

\section{OBJECT IDENTIFICATION}

In this chapter the algorithms used to identify the basic objects needed to perform this analysis are discussed. All identification algorithms exist within the common $\mathrm{D} \varnothing$ computing framework called vjets_cafe. It is a database of packages that are used and maintained by the entire collaboration.

\subsection{Electron Identification}

The first step of electron identification is to measure the energy of each tower (see Section 2.2.2). The tower with the highest $E_{T}(E \sin \theta)$ becomes the "seed" and

all towers within $\Delta R=\sqrt{(\Delta \phi)^{2}+(\Delta \eta)^{2}}<0.2$ are summed to create a cluster. If the calorimeter cluster can be matched to a track from the SMT or CFT it is identified as an electron rather than a photon, as photons generally do not leave a signature in the tracker. Information from the CPS detector is used to recalculate the total energy, while tracking information provides a measure of the electrons transverse momentum. Several additional variables help identify electrons.

- EM fraction $f_{e m}$ - The electromagnetic fraction (emf) is the ratio of energy deposited in the EM calorimeter to the total energy deposited in the EM plus hadronic calorimeters. It is most useful in rejecting $\pi^{0}$ 's and other light hadronic particles. For real electrons this ratio should be close to unity.

$$
f_{e m}=\frac{E_{e m}(\Delta R<0.2)}{E_{\text {tot }}(\Delta R<0.2)}
$$

- Isolation - Isolation looks for clusters that do not overlap in $\eta-\phi$ space. It requires that a fraction of the total energy in the cluster of $\Delta R<0.4$ to not be 
outside a cluster of $\Delta R<0.2$. Isolation helps remove detector noise, or poorly reconstructed electrons.

$$
f_{\text {iso }}=\frac{E_{\text {tot }}(\Delta R<0.4)-E_{\text {em }}(\Delta R<0.2)}{E_{\text {tot }}(\Delta R<0.4)}
$$

- Spacial Track Match - The quality of the fit for the $\phi$ and $z$ coordinates as measured by the tracker and calorimeter is defined as $\chi^{2}$ :

$$
\chi_{\text {spacial }}^{2}=\left(\frac{\Delta \phi}{\sigma_{\phi}}\right)^{2}+\left(\frac{\Delta z}{\sigma_{z}}\right)^{2}+\left(\frac{E_{T} / p_{T}-1}{\sigma_{E_{T} / p_{T}}}\right)
$$

$\Delta \phi$ and $\Delta z$ are the differences as measured by the tracker versus calorimeter, $\sigma_{\phi}$ and $\sigma_{z}$ are the RMS of these differences. The final term constrains the calorimeter energy to the transverse momentum measured by the tracker of objects in the CC.

- H-Matrix - The HMx7 is a 7 x 7 matrix comprised of seven correlated variables that describe the evolution of the shower shape within the EM calorimeter: (1-4) the fraction of energy deposited to the total in each of the four EM layers, (5) the shower width in $\Delta R,(6)$ the log of the total shower energy, and (7) the $z$ position of the PV. Another matrix, HMx8, includes the transverse shower width. The EM shower shape differs from hadronic deposits in the EM calorimeter and again helps reject hadronic particles.

- Electron Likelihood - The most discerning of these variables is calculated from probabilities of eight variables: (1) emf, (2) $\mathrm{HMx} 7$, (3) $\mathrm{HMx} 8$, (4) $E_{T} / p_{T}$, (5) total track $p_{T}$ in a cone $\Delta R<0.4,(6)$ number of tracks in cone $\Delta R<0.05$, (7) spacial track match, $\chi^{2}$, and (8) distance to closest approach (DCA - the shortest distance from the selected track to the line parallel to the $z$-axis which 
passes through the primary vertex position). Objects with high $\mathcal{L}_{e}$ are very likely to be electrons.

$$
\mathcal{L}_{e}=\frac{\prod_{i} P_{\text {sig }, i}\left(x_{i}\right)}{\prod_{i} P_{s i g, i}\left(x_{i}\right)+\prod_{i} P_{b k g, i}\left(x_{i}\right)}
$$

The final electron identification algorithm combines these and other variables in a multivariate discriminant. A sample of $Z \rightarrow e e$ events from data is trained against multi-jet background also from data. The output of the electron MVA, $\xi_{e}$, has several predefined selection requirements that have similar efficiencies as previous DØ working points but much better background rejection. Use of this identification variable is one of the major improvements to the event selection made in this analysis.

\subsection{Tau Identification}

Hadronic tau decays are of particular interest in collider experiments because they constitute the majority of tau decays. A hadronic decay will leave at least one track in the inner detector and energy in the hadronic calorimeter. This energy is reconstructed as a narrow jet with $\Delta R<0.3$. The presence of the neutrino will also lead to an imbalance in transverse energy, known as missing transverse energy or $\mathbb{E}_{T}$. If the hadronic decay contains one or more $\pi^{0}$ 's, there will also be energy deposited in the electromagnetic calorimeter. It is essential to define three tau "types": type 1, type 2 and type 3 . The physical motivation behind the types roughly corresponds to the number of "prongs" in the decay, but the distinction is largely made by the signature each type leaves in the detector. The tau types are summarized in Tab. 3.1.

As discussed in Section 1.2, tau leptons can decay hadronically or leptonically. Leptonic decays of taus are indistinguishable from prompt decays, into electrons or muons, thus identification of taus implies hadronic decays which have difficult signatures to differentiate particularly from jets or electrons. Information from the tracker 
Table 3.1. Detector driven tau decay types, with branching ratios and detector signatures.

\begin{tabular}{cccc}
\hline \hline$\tau$ type & Physical Process & BR & Detector Signature \\
\hline 1 & $\tau^{ \pm} \rightarrow \pi^{ \pm} \nu_{\tau}$ & $10.9 \%$ & one CAL cluster, one track \\
2 & $\tau^{ \pm} \rightarrow \rho^{ \pm}\left(\rightarrow \pi^{0} \pi^{ \pm}\right) \nu_{\tau}$ & $36.5 \%$ & one CAL cluster, at least one electromagnetic \\
& $\tau^{ \pm} \rightarrow\left(\geq 2 \pi^{0}\right) \pi^{ \pm} \nu_{\tau}$ & & $\begin{array}{c}\text { associated subcluster, one track } \\
3\end{array}$ \\
$\tau^{ \pm} \rightarrow a_{1}^{ \pm}\left(\rightarrow \pi^{ \pm} \pi^{\mp} \pi^{ \pm}\right) \nu_{\tau}$ & $13.9 \%$ & $\begin{array}{c}\text { one CAL cluster, more than one track, with } \\
\text { or without an associated EM subcluster }\end{array}$ \\
\hline \hline
\end{tabular}

and both EM and hadronic calorimeters is used in tau identification. Tau candidates $\left(\tau_{\text {cand }}\right)$ are identified by three criteria:

- Hadronic Cluster - Uses a cluster of size $\Delta R<0.5$ to find the total hadronic energy deposited.

- EM Cluster - Uses the Nearest Neighbor Algorithm starting with the finely segmented third layer EM calorimeter to identify the $\pi^{0} \rightarrow \gamma \gamma$ events possibly present in the decay. The EM sub-cluster should have $E>800 \mathrm{MeV}$.

- Tracks - All tracks that point to the calorimeter cone within $\Delta R<0.5$ and $p_{T}$ $>1.5 \mathrm{GeV}$ are sorted by $p_{T}$. If either the second or third tracks have $\Delta z$ from the primary vertex less than $2 \mathrm{~cm}$ they are retained. Finally, if the invariant mass of the first two tracks is at least $1.1 \mathrm{GeV}$, the first and second are retained, and if the invariant mass of all three is less then $1.7 \mathrm{GeV}$ (the mass of the tau) all are retained.

For most other particles this criteria would be enough to have a relatively pure sample, however many jets and electrons easily pass these requirements so a more sophisticated identification is required. A neural network, $\mathrm{NN}_{\tau}$, is trained to separate signals (taus) from background (multi-jets and electrons). 12 variables that use information from the coarse and fine hadronic calorimeter, the EM calorimeter 
and the tracker are used as input variables to the $\mathrm{NN}_{\tau}$. They include isolation, shower composition, shower shape and correlations between the calorimeter and tracker.

Objects that are determined to be more tau-like are assigned values closer to unity. A selection on each output around 0.9 defines a "good" tau. Even after selecting high quality taus, the contamination from electrons in type 2 taus is significant. Another neural network, $\mathrm{NN}_{\tau / e}$, is created only for type two taus. The output of both $\mathrm{NN}_{\tau}$ and $\mathrm{NN}_{\tau / e}$ can be seen in Fig. 3.1. In Fig. 3.1 (a), (b) and (c) the concentration

of $Z \rightarrow \tau \tau$ near +1 is clear. The majority of the multi-jet contribution has already been removed during the certification process, but it is clear that the MJ contribution increases as $\mathrm{NN}_{\tau}$ decreases. In Fig. 3.1 (d) the contribution of $Z \rightarrow e e$ in the left most bins is dominant, and removal of these events greatly decreases the contamination of electrons in type 2 taus.

A "pseudo" tag-and-probe method is used to calculate efficiencies of the $\mathrm{NN}_{\tau}$. Data containing exactly one muon and one tau with several fiducial selections are identified and used to perform the tau ID certification. The "tag" is the muon which is easy to identify relative to the hadronic tau and has a known, small fake rate. The "probe" is the hadronic tau. The result is a sample about $70 \%$ pure in $Z \rightarrow \tau \tau$. Contributions from $Z \rightarrow \mu \mu$ (where one muon is not reconstructed and a jet fakes a tau), $W \rightarrow \ell \nu, t \bar{t}$, and di-boson contribute about $15 \%$, and the remaining background $(\sim 15 \%)$ is multi-jet.

\subsubsection{Tau ID Certification with the New $\mathrm{NN}_{\tau}$}

In 2010 a new $\mathrm{NN}_{\tau}$ was developed by previous tau ID group members [25]. The goal was to achieve significant hadronic tau identification improvement by making several small modifications. First, tuning of the multivariate parameters and training $\mathrm{NN}_{\tau}$ in two separate $p_{T}$ bins $\left(p_{T}<45\right.$ or $\left.p_{T}>45 \mathrm{GeV}\right)$ brought a few percent improve- 

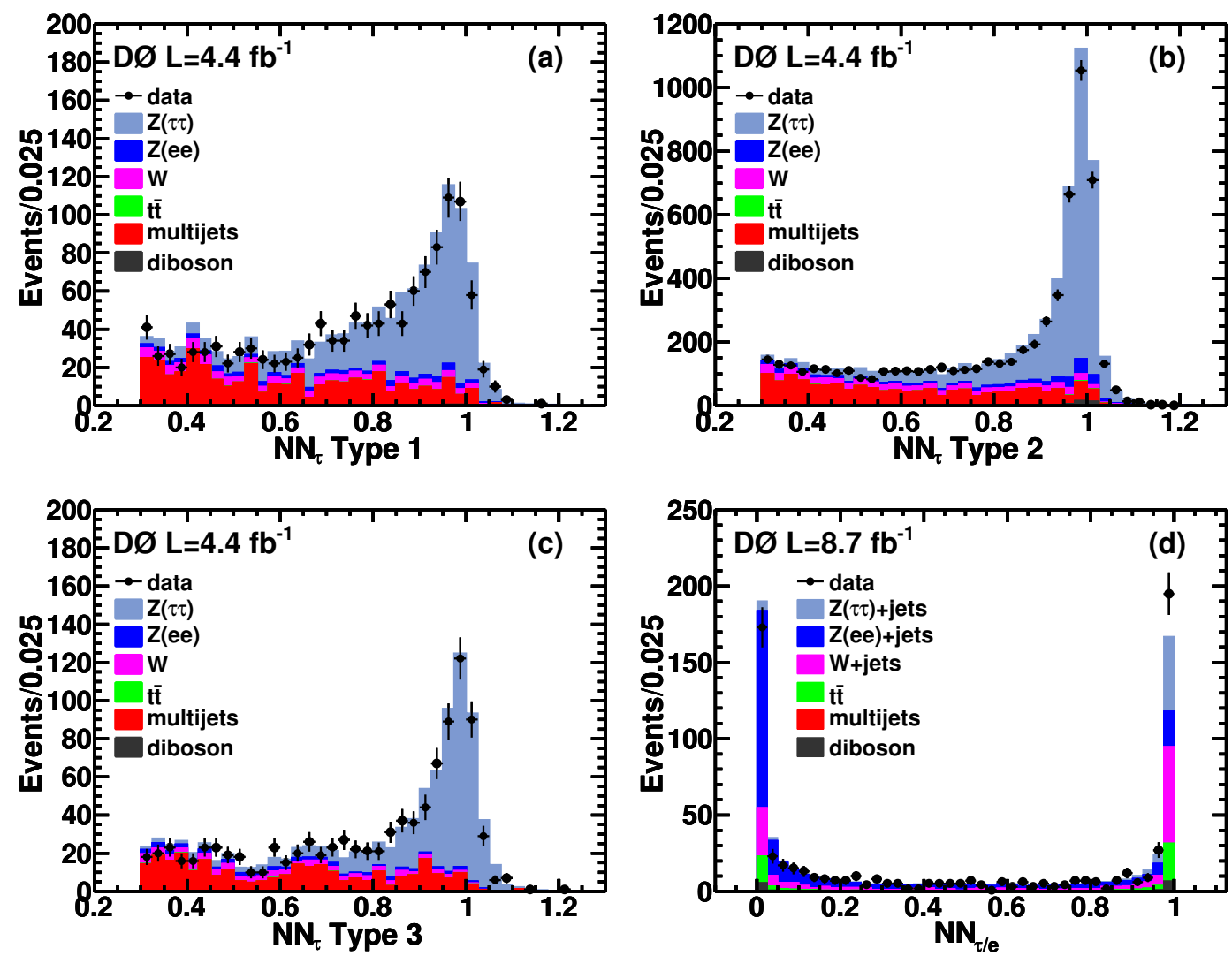

Figure 3.1. (a), (b) and (c) sample $\mathrm{NN}_{\tau}$ distributions by type and (d) $\mathrm{NN}_{\tau / e}$. The contribution from real taus from $Z \rightarrow \tau \tau$ is concentrated towards +1 in each distribution.

ment for all tau types. Secondly, central pre-shower (CPS) detector information was added to aid in type 2 tau identification. The CPS helped improve electron/photon identification, hence it could help identify the decay of $\pi^{0} \rightarrow \gamma \gamma$ present in type 2 decays. However, no significant improvement was found. Because the tau has a short lifetime secondary vertexing is possible for type 3 taus since they have two or more tracks. $b$-tagging information was used and showed a several percent improvement in identification. Finally, a modest improvement was obtained by handling type 1 taus separately based on whether or not they were located in the inter cryostat region (ICR) $1.0<\eta<1.4$ because of poor EM calorimeter coverage. 
The tau ID efficiency is derived as a function of the $\mathrm{NN}_{\tau}$ selection and is shown in Fig. 3.2. The efficiency curves are nearly identical because of the similarities between the old and new $\mathrm{NN}_{\tau}$, however, the overall event yield for the new $\mathrm{NN}_{\tau}$ increased by about $10 \%$. Table 3.2 shows the yields for all tau types for the Run IIb3+4 portion of the tau identification. Careful examination of this table will however show that there is only a $6 \%$ increase of $Z \rightarrow \tau \tau$ events, whereas there is about a $27 \%$ increase of MJ events. The impact of this on the erjj analysis is discussed in Section 4.6.1.

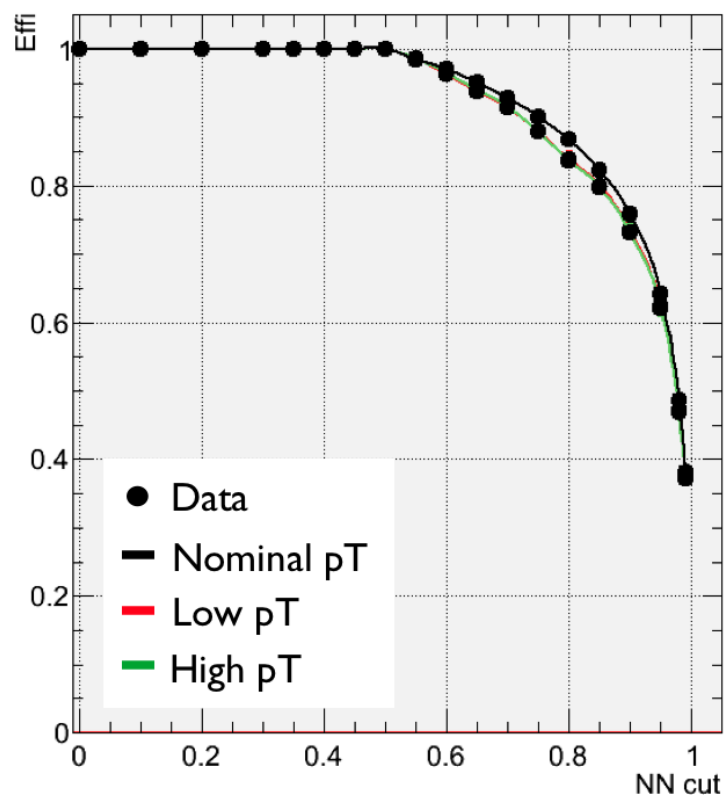

(a)

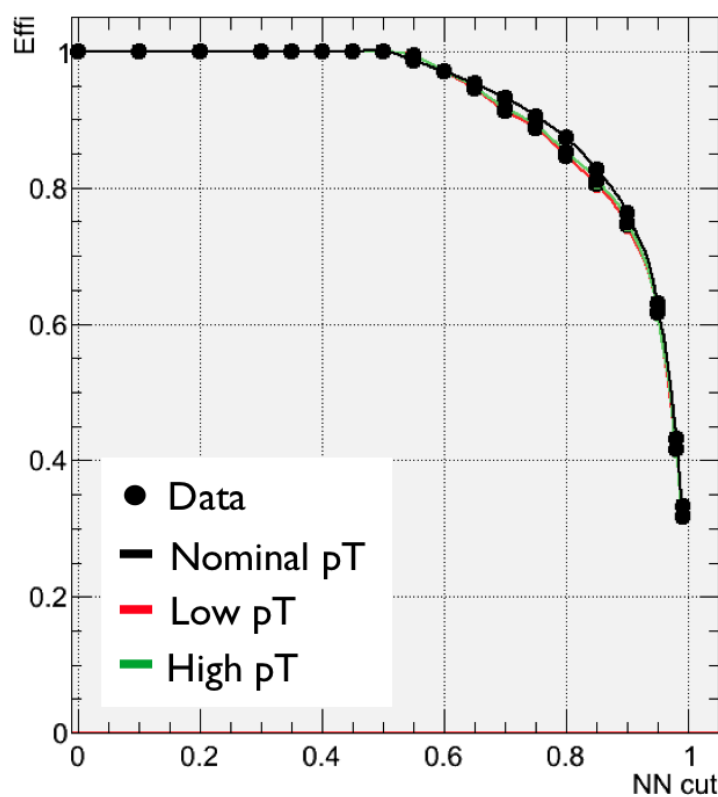

(b)

Figure 3.2. Tau ID efficiency curve for type 2 taus, (a) old $\mathrm{NN}_{\tau}$ and (b) new $\mathrm{NN}_{\tau}$. The green and red lines show the efficiency for different $p_{T}$ selections used to calculate systematic uncertainties.

\subsection{Jet Identification}

Jets originate from either quarks or gluons and are simply a large number of tracks and calorimeter energy deposited from a process known has hadronization. 
Table 3.2. Tau ID event yields and uncertainty for the old (left) and new (right) $\mathrm{NN}_{\tau}$ for Run IIb3+4.

\begin{tabular}{c|ccc|ccc}
\hline \hline Sample & Events & & Uncertainty & Events & & Uncertainty \\
\hline$Z \rightarrow \tau \tau$ & 3574.7 & \pm & 13.1 & 3809.9 & \pm & 13.6 \\
$Z \rightarrow \tau \tau+2 c$ & 37.2 & \pm & 0.3 & 39.6 & \pm & 0.3 \\
$Z \rightarrow \tau \tau+2 b$ & 16.1 & \pm & 0.1 & 17.1 & \pm & 0.1 \\
$Z \rightarrow \mu \mu$ & 268.9 & \pm & 4.0 & 295.6 & \pm & 4.3 \\
$Z \rightarrow \mu \mu+2 c$ & 5.1 & \pm & 0.2 & 5.9 & \pm & 0.2 \\
$Z \rightarrow \mu \mu+2 b$ & 1.8 & \pm & 0.0 & 2.0 & \pm & 0.0 \\
$W \rightarrow \ell \nu$ & 274.0 & \pm & 4.9 & 336.7 & \pm & 5.5 \\
$W \rightarrow \ell \nu+2 c$ & 6.4 & \pm & 0.3 & 7.6 & \pm & 0.3 \\
$W \rightarrow \ell \nu+2 b$ & 2.1 & \pm & 0.1 & 2.6 & \pm & 0.2 \\
$t \bar{t}$ & 15.8 & \pm & 0.2 & 10.7 & \pm & 0.2 \\
diboson & 42.1 & \pm & 0.9 & 43.4 & \pm & 0.9 \\
MJ & 595.6 & \pm & 28.1 & 757.7 & \pm & 31.2 \\
\hline Exp. Bkg & 4839.8 & \pm & 31.7 & 5328.9 & \pm & 34.7 \\
Data & 4654 & \pm & 50.6 & 5185 & \pm & 54.0 \\
\hline \hline
\end{tabular}

After a high energy collision, quark-antiquark pairs are produced from the vacuum energy, which subsequently decay into "showers" of particles mostly made of protons, neutrons, pions, kaons, and photons. In all hadron colliders it is difficult to identify and measure the energy of jets correctly because of the complexity of their detector signature.

\subsubsection{Jet Algorithms}

To measure the energy and momentum of these objects the DØ Run II Cone Algorithm is used [26]. There are four basic steps to this algorithm: (1) pre-clustering, (2) clustering, (3) adding midpoints and (4) merging/splitting.

- Pre-clustering - uses a Simple Cone Algorithm to find seeds for the Run II Cone Algorithm. It loops over objects with $p_{T}>500 \mathrm{MeV}$. It adds objects within a cone $\Delta R<0.3$ and $p_{T}<1 \mathrm{MeV}$. After looping over all possible 
objects with $p_{T}>500 \mathrm{MeV}$, all objects with $p_{T}<1 \mathrm{GeV}$ are removed, and the remaining items become seeds for the Run II Cone Algorithm.

- Clustering - uses two input lists: the seeds from pre-clustering, and the list of clusters of items which are used to form proto-jets. Typically a cone of $\Delta R<0.5$ defines the size of a jet, but any size can be specified. The distance $\Delta R_{\text {min }}$ between the two lists is calculated, and if $\Delta R_{\min }>R_{\text {cone }} / 2$ the pre cluster is considered a seed for the proto-jet candidate. The process is repeated until one of three stability requirements is met.

- Midpoints - To avoid contamination from soft radiation, proto-jets are also searched for at midpoints between two proto-jets. The midpoint proto-jet candidate must satisfy $R_{\text {cone }}<\Delta R<2 R_{\text {cone }}$. The list of these candidate events is used in the final step.

- Merging/Splitting - is used to ensure that jets are not double counted from the list of proto-jets from midpoints and from clustering. If adjacent protojets share more than $50 \%$ of their energy, they are merged, otherwise they are split. This process iterates until there are no more proto-jets remaining. The merged/split objects are the jets used in analyses.

\subsubsection{Jet Energy Scale}

The measured energy of jets does not generally correspond to the actual initial energy of the parent particle. The calorimeter has a different response for each type of particle, and energy can be lost in dead areas. The energies are corrected on a jet-by-jet basis using the Jet Energy Scale (JES) [27].

$$
E_{j e t}^{c o r r}=\frac{E_{j e t}^{\text {meas }}-E_{0}}{R_{\text {jet }} k_{R} S_{j e t}} k_{0}
$$


In Eq. 3.5, $E_{j e t}^{\text {corr }}$ is the corrected jet energy used by physics analyses, $E_{j e t}^{\text {meas }}$ is the measured jet energy, $E_{0}$ is the energy offset from electronic noise and pile-up collisions, $R_{\text {jet }}$ is the average calorimeter response to the energy deposited by the particles that make up the jet, $S_{j e t}$ corrects for the migration of energy into and out of the jet cone, and $k_{0}$ and $k_{R}$ are correction factors for biases in $E_{0}$ and $R_{j e t}$ respectively.

\subsection{Missing Energy Identification}

Neutrinos cannot be directly measured by the $\mathrm{D} \varnothing$ detector since they interact only via the weak force. Their presence is inferred as an imbalance in the transverse energy of an event as measured by the EM and fine hadronic calorimeter. Due to the higher noise level, the coarse hadronic are not included. The missing energy (missing $E_{T}, \mathbb{E}_{T}$ or MET) is the negative of the vector sum of all transverse energy. The $x$ or $y$ components are given by

$$
\mathscr{E}_{x, y}=-\left(\sum_{\text {all cells }} E_{x, y}-\sum_{\text {phys cells }} E_{x, y}^{\text {phys cells }}+\sum_{\text {phys obj }} E_{x, y}^{\text {phys obj }}\right)
$$

where the physical energy of each cell $\left(E^{\text {phys cells }}\right)$ is subtracted, and replaced by the offline calculated energy of the physics object $\left(E^{\text {phys obj }}\right)$. These components are then added in quadrature to give the total missing energy.

$$
\mathbb{E}_{T}=\mathbb{E}_{x} \oplus \mathbb{E}_{y}
$$

An important companion to the calculation of $\mathbb{E}_{T}$ is the calculation of the missing $E_{T}$ significance:

$$
\mathcal{S}=2 \log \left(\frac{\mathbb{E}_{T}}{2 \sigma}\right)^{2}
$$


where $\sigma$ is the variance on the probability distribution of a unit vector in the direction of the measured $\mathbb{E}_{T}$ [28]. For processes like multi-jet the $\mathcal{S}$ is low $(\lesssim 1)$ because the missing energy arises from detector mismeasurement. The $\mathbb{E}_{T}$ significance for $Z \rightarrow e e$ would also be low because there are no neutrinos present in such decays and again the missing energy arises from detector mismeasurement. For $Z \rightarrow \tau \tau$ or $t \bar{t}$ there are neutrinos present so $\mathcal{S}$ is much higher $\sim 6$.

\subsection{Muon Identification}

The first step of muon identification requires a hit in each of the three layers of the muon system, and also requires a CFT/SMT track matched to those hits. Out of time hits are rejected to avoid accepting cosmic ray muons, or radiation noise from extraneous sources such as beam gas collisions or a shower of particles arising from a halo proton hitting the beam pipe. The central tracker has better resolution than the muon system and helps measure muon momenta. 


\section{CHAPTER 4}

\section{EVENT SELECTION}

\subsection{Data}

The data used in this analysis were collected from 2002 to 2011 during Run II at the Fermilab Tevatron using $p \bar{p}$ collisions at $\sqrt{s}=1.96 \mathrm{TeV}$ and total $9.7 \mathrm{fb}^{-1}$. Run II is divided into four different epochs, Run IIa, Run IIb1, Run IIb2, Run IIb3 and Run IIb4. Small changes to the Tevatron and DØ necessitated this delineation. The data are first skimmed by the DØ Common Samples Group into a collection of samples containing at least one good quality electron with $p_{T}>12.5 \mathrm{GeV}$. Together these samples are known as the "EMinclusive" sample and are collectively well over 100 TB (see Appendix A). The EMinclusive samples are skimmed again requiring at least one hadronic tau decay with $p_{T}>12.5 \mathrm{GeV}$. Taus below this threshold are not certified by the Tau ID group and therefore have unknown efficiencies. It is on this data sample that the selections discussed in Sections 4.4 - 4.6 are applied.

The data are subject to standard $\mathrm{D} \varnothing$ data quality requirements, that remove bad events as defined by the CFT, SMT, Calorimetry, and Muon groups. Bad runs usually correspond to times when key detector components were offline or malfunctioning, and represent about $10 \%$ of the delivered data. Figure 4.1 shows the percentage of data lost by subsystems for all of Run II. There is a clear trend of the percentage loss downward which is a tribute to the diligent work of the collaboration over many years. 
Luminosity Loss Run2 : 19 April 2002 -> 30 September 2011

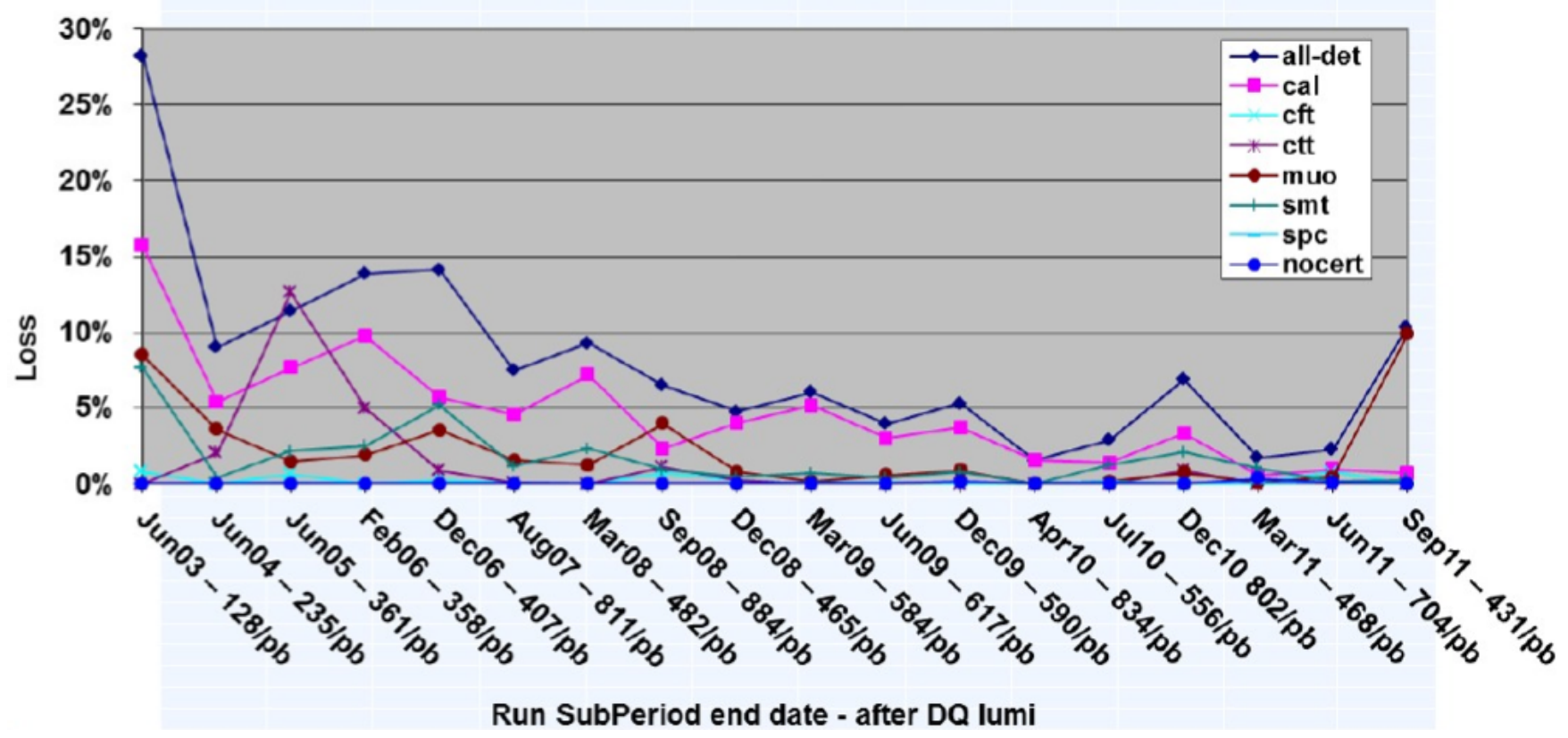

Figure 4.1. Percent of data loss by detector subsystem. Partial outages in the calorimeter are the major source of loss.

\subsection{Trigger}

This analysis utilizes a suite of triggers aimed at being as inclusive as possible. The EJetsOR trigger is the logical OR of over 50 individual triggers. These individual triggers include single electron, single jet, electron-jet, and electron-tau. The EJetsOR trigger efficiency is calculated using the tag-and-probe method on a $Z \rightarrow$ ee sample by the EM ID group. Figure 4.2 shows the electron $p_{T}$ trigger efficiency curves in the central and end calorimeters for three slightly different versions of the trigger list. The efficiency is higher in the central calorimeter because the tag-and-probe method is easier to execute in this region.

In previous versions of this analysis [29] a SingleEM trigger, which as indicated by the name demands at least one electron with $E_{T}$ above threshold, was used. Studies were performed to include an inclusive trigger approach where the Monte 


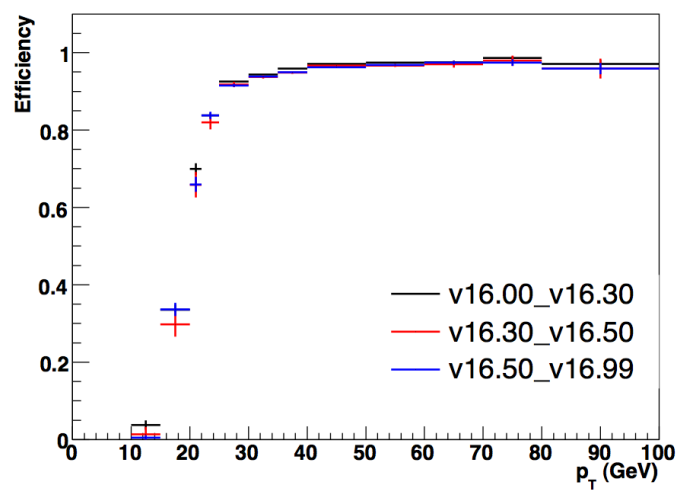

(a)

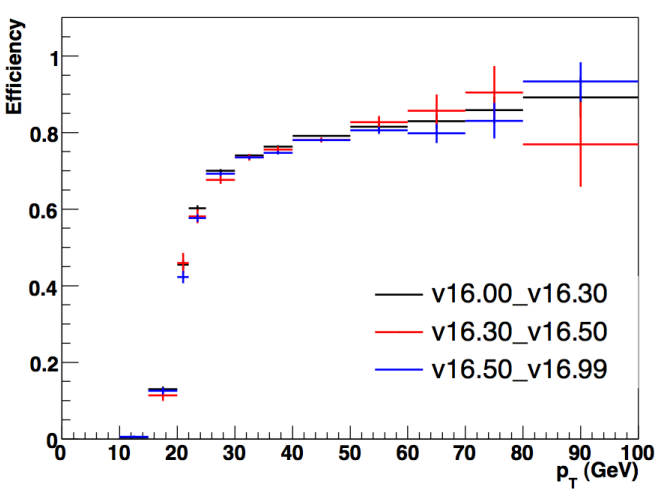

(b)

Figure 4.2. Electron $p_{T}$ trigger efficiency curves for the EJetsOR trigger in the (a) central calorimeter and (b) the end calorimeter for slightly different trigger lists. The tag-and-probe method is more difficult in the end calorimeter and causes a slight drop in efficiency.

Carlo backgrounds were scaled according to the ratio of all triggered events to single electron events. The modeling using this scheme was poor. The EJetsOR trigger increases the number of accepted events compared to the SingleEM trigger, and in addition has better modeling. Figure 4.3 shows the shape differences of electron $p_{T}$ when different triggers are used. For the inclusive trigger there are clearly more low $p_{T}$ events, and a larger multi-jet (red) contribution which is difficult to model.

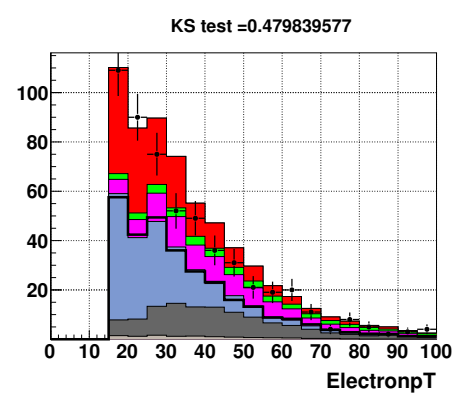

(a)

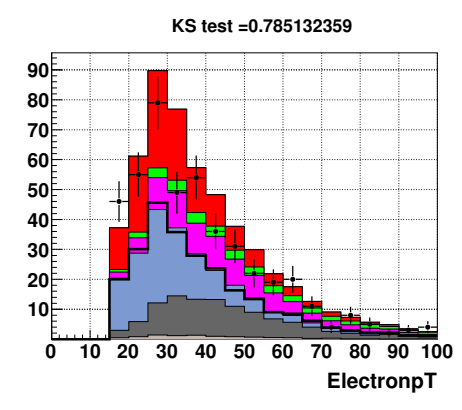

(b)

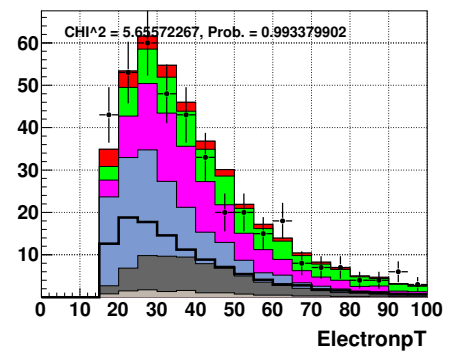

(c)

Figure 4.3. Electron $p_{T}$ for three different triggers (a) inclusive, (b) SingleEM and (c) EJetsOR. The trigger turn-on is visible in (b) and (c) and ultimately (c) was chosen as a compromise between increased yield and quality modeling (see Fig. 4.4 for the legend). 


\subsection{Backgrounds}

Understanding all the processes that result in the same final state as the searched for signature is critical to this analysis. Several types of backgrounds exist. (1) Irreducible backgrounds have the exact same physical final states as the signal channel, for this analysis $Z \rightarrow \tau \tau$. (2) Fake backgrounds occur when some physical process produces a portion of the final state, but some objects are misidentified. $Z \rightarrow e e$ is an example here where one electron is misreconstructed as a tau. Also $W+$ jets, $t \bar{t}$, and di-boson (DB) all have objects that fake a portion of the final state signature. (3) Instrumentally induced backgrounds caused by detector effects. This process is called multi-jet (MJ) and consists of quark or gluon jets faking all final state objects. The legend for all of the data/MC plots is shown in Fig. 4.4. The sum of all signals is shown by the solid black line and is multiplied by 250 to make the distribution visible.

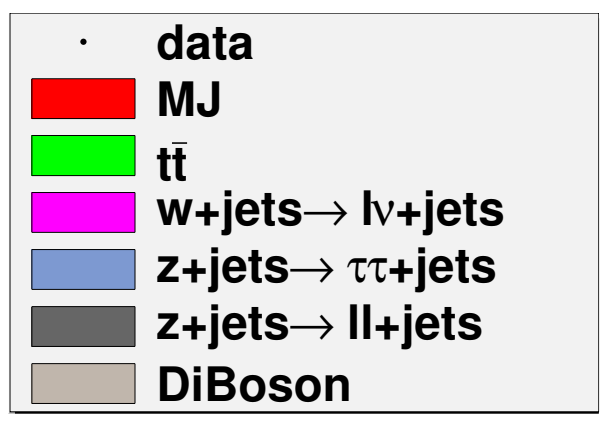

Figure 4.4. Legend for all plots in this paper. Signals are shown in a solid black lines multiplied by 250 .

\subsection{1 $Z+$ jets}

The irreducible background is $Z \rightarrow \tau \tau$. Rather than producing a Higgs, a $Z$ is produced and subsequently decays into a pair of taus. Additionally at least 2 other 
jets are present from initial or final state radiation. Except for the invariant mass the $Z \rightarrow \tau \tau$ background has an extremely similar signature as signal. An example showing how similar these shapes are is in Fig. 4.5. While other backgrounds can clearly be distinguished, the shape of signal (black line) and $Z \rightarrow \tau \tau$ (light blue) are very similar.

Since event selection is heavily reliant on electron selection the most pervasive background in this analysis is from $Z \rightarrow e e$ events. In this background a $Z$ boson decays to two electrons; one electron is reconstructed properly, and the other is misidentified as a tau which happens quite easily. Since the tau-electron fake rate is fairly high this background is difficult to combat. The $Z \rightarrow \mu \mu$ background in the $\mu \tau j j$ analysis is much easier to remove since the tau-muon fake rate is very low.

One of the principle ways of suppressing the $Z \rightarrow e e$ is by examining $\mathbb{E}_{T}$. No neutrinos are present in $Z \rightarrow e e$ events so $\mathbb{E}_{T}$ must arise from other effects (i.e. jet energy mismeasurement), as opposed to $Z \rightarrow \tau \tau$ where 2 neutrinos truly carry away some energy. See Section 4.4.5 for detailed $\mathbb{E}_{T}$ selections.

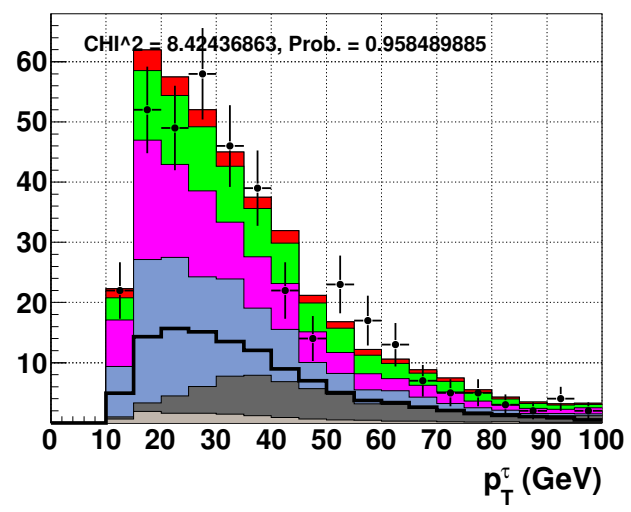

Figure 4.5. Comparing the shape similarity of signal (black line) with $Z \rightarrow \tau \tau$ background (blue) in the tau transverse momentum distribution. 


\subsubsection{W+jets}

This background arises when a $W$ boson is produced and decays into either an $e$ or tau, and a jet fakes the other lepton ( $e$ or tau). Again, two or more jets are also present as shown in the Feynman diagram for this process in Fig. 4.7 (a). The $\mathbb{E}_{T}$ present in these events is real and behaves similarly to signal. The modeling of this background can often be difficult, so an orthogonal control sample is created by making the following selections:

- $0.3<\mathrm{NN}_{\tau}<0.8$

- $p_{T}^{e}>30 \mathrm{GeV}$

- $\mathbb{E}_{T}>30 \mathrm{GeV}$

The resulting control sample is $67 \% \mathrm{~W}+$ jets and $20 \% t \bar{t}$. Figure 4.6 shows that several fundamental variables from the $W+$ jets control sample are well modeled.

\subsection{3 t $t \bar{t} /$ di-boson}

Figure 4.7 (b) shows $t \bar{t}$ production and decay into e $\tau j j$ final states. In this background each $t$-quark decays into a $W$ boson and $b$-quark pair, where the $W$ 's give the leptons and the $b$ 's give two jets. The di-boson background is pair production of vector bosons either $W+W$ or $Z+Z$ or $W+Z$ in the presence of other jets. It contributes only a few percent to the total background and is shown in Fig. 4.7 (d). It is interesting to note, that years after the top quark was discovered at Fermilab it now represents a background to an even more rare process.

\subsubsection{Multi-jet}

The multi-jet (MJ) background is instrumentally induced and shown in Fig. 4.7 (c). It arises when jets fake both a tau and an electron and two additional jets are identified. MJ is estimated from data and is addressed in detail in Section 4.5. 


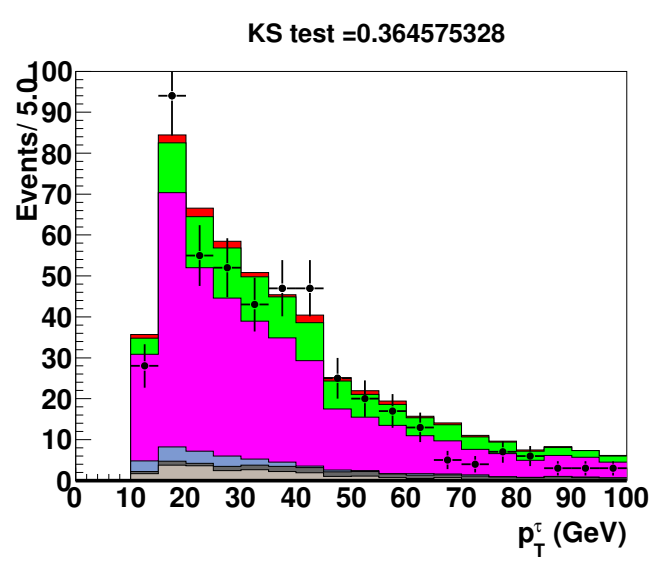

(a)

KS test $=0.052509902566077293$

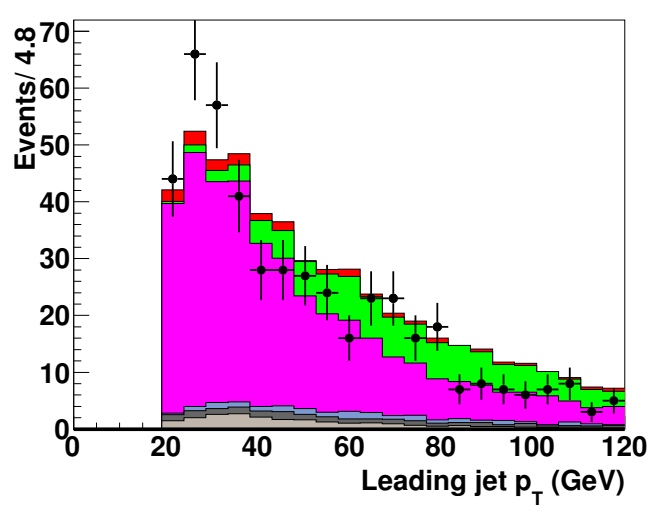

(c)

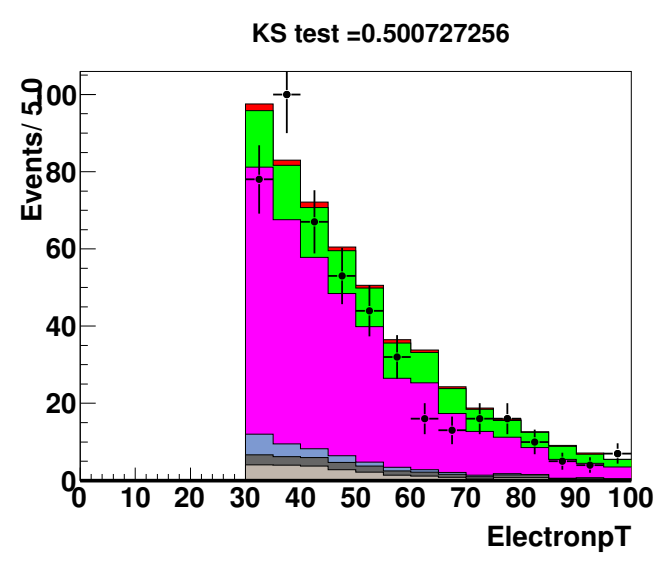

(b)

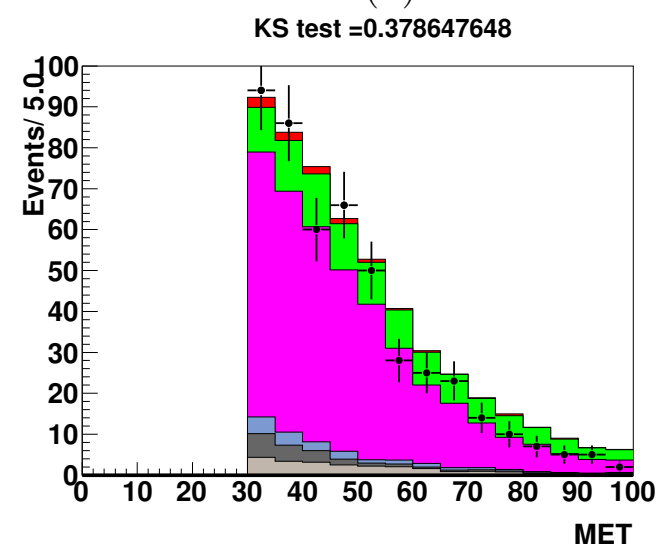

(d)

Figure 4.6. $W+$ jets control sample plots used to check the modeling of this background. Most variables show decent agreement.

\subsection{Object Selection}

In this section the selection requirements of this analysis are presented. The general description of object identification is discussed in Chapter 3. Extensive optimizations were performed for these selections in conjunction with optimizations of the multivariate technique discussed in Chapter 5. There are four principles objects in this analysis, an electron, a hadronic tau decay, and two jets. There are several general requirements involving multiple objects. 


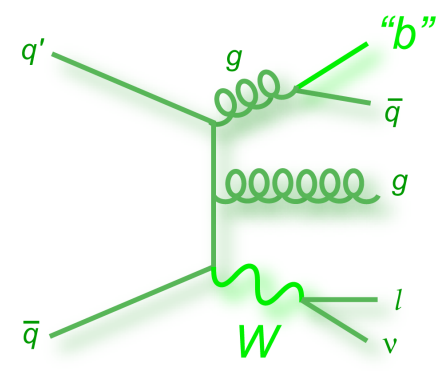

(a)

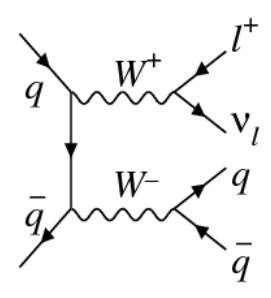

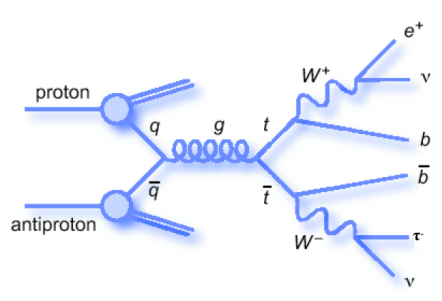

(b)

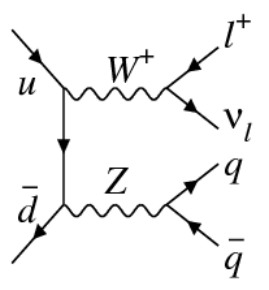

(d)

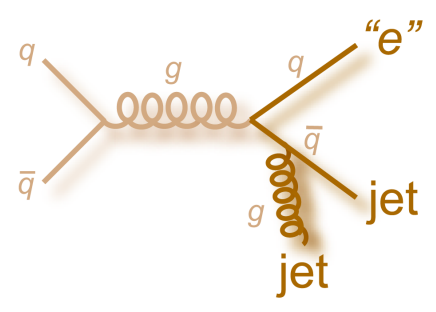

(c)

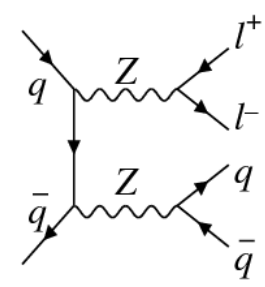

Figure 4.7. Feynman diagrams for four of the background processes. (a) $W+$ jets, (b) $t \bar{t}$ and (c) multi-jet and (d) di-boson.

- The tau and electron are required to have opposite charge, since they should originate from a neutral Higgs.

- $\Delta R(e, j)<0.5$ ensures that the electron and jet are not reconstructed as the same object for that particular event.

\subsubsection{Electron Selection}

Electrons are primarily selected through the use of the multivariate output, $\xi_{e}$. Electrons must satisfy $\xi_{e}>-0.96(-0.74)$ in the $\mathrm{CC}(\mathrm{EC})$, a standard $\mathrm{D} \varnothing$ working point called "emvPoint05(1)_eff". Electrons in the EC are not required to have a track matched to the calorimeter deposit, since very forward tracking does not always reliably match tracks to electrons. Better performance is achieved by using the electron MVA to reject electron fakes in the EC than via track matching 
requirements. Electrons are required to have: $p_{T}^{e}>20 \mathrm{GeV}$ below which modeling can be unreliable, and subject to the standard requirement to not be in the ICR region: $|\eta|<1.1$ or $1.5<|\eta|<2.5$, where there is limited EM calorimeter coverage.

\subsubsection{Tau Selection}

Various fiducial selections are made to identify a high quality tau. Many of these conditions are designed to reduce the MJ background which overwhelms tracking and energy measurements for low $p_{T}$. The requirements are as follows:

- Taus must have $\mathrm{NN}_{\tau}>0.92 / 0.90 / 0.91$ for type $1 / 2 / 3$. The values vary slightly to match the magnitude of the fake rates between types.

- $p_{T}^{\tau}>12.5 / 12.5 / 15.0 \mathrm{GeV}$ for type $1 / 2 / 3$ and $|\eta|<2.0$. These are nominal selections based on the tau ID. The $p_{T}$ for type 3 is slightly higher because there are several tracks contained in this decay.

- $p_{T}^{\text {trk }} / E_{T}^{\tau}$ is required to be greater than $(0.65 / 0.5 / 0.5)$ for type $1 / 2 / 3$, this primarily removes muons faking tau's.

- The track $p_{T}$ must exceed $7 \mathrm{GeV}$ for type 1 taus and $5 \mathrm{GeV}$ for type 2 taus. For type 3 taus at least one track must have $p_{T}>5 \mathrm{GeV}$ and the sum of all tracks must exceed $\Sigma p_{T}^{i}=7 \mathrm{GeV}$. By definition type 3 taus have more tracks, and should therefore have more total momentum.

- Taus that have $\Delta R(\tau, e)<0.1$ or $\Delta R(\tau, j)<0.1$ are removed. This processes is called electron-tau or tau-jet matching and assures that objects in the event are not reconstructed as both taus and electrons or jets.

- Type 3 taus with exactly two tracks, for which the sign of the tau is ambiguous are removed.

With only these selections the $Z \rightarrow e e$ background would still overwhelm the SM signal prediction for this analysis. A number of selections were optimized to 
provide the best modeling for the multivariate analysis, and to increase the signal significance.

- Type 1 taus are removed from the inter-cryostat region (ICR), $1.05<|\eta|<1.5$ (Fig. 4.8). This selection is used to reject $Z \rightarrow e e$ background where one of the electrons has been reconstructed as a tau in the ICR region, where there is poor EM calorimeter coverage.

- Type 2 taus must have $\mathrm{NN}_{\tau / e}>0.5$, which helps reject some $Z \rightarrow$ ee background. This selection, which was lowered from 0.95 in previous iterations of this analysis, was unnecessarily stringent. Loosening this selection and allowing the multivariate to handle the background is preferred to simply eliminating it with a tight selection.

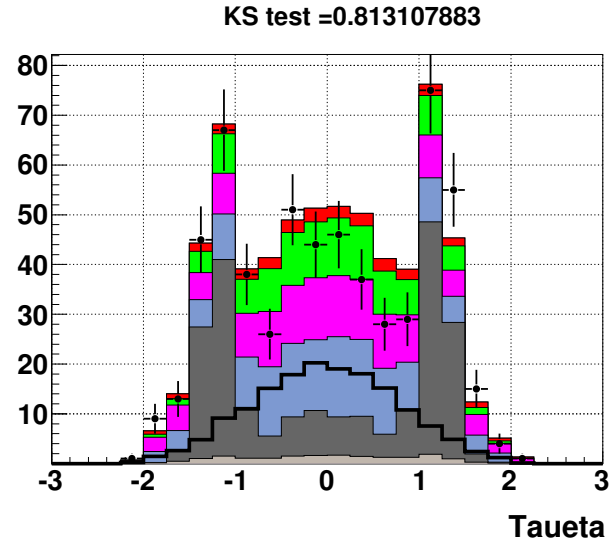

(a)

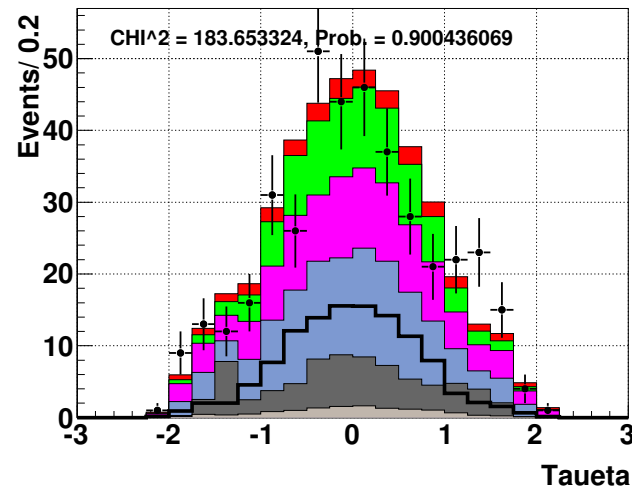

(b)

Figure 4.8. The $\eta^{\tau}$ distributions (a) without the type 1 ICR removal and (b) with the removal. Electrons entering this region of the detector are reconstructed as taus.

\subsubsection{Missing Mass Calculator}

Computing the invariant mass from the decay of a pair of tau leptons is notoriously difficult because of the presence of multiple neutrinos. Often the taus are 
produced "back-to-back" and the $\mathbb{E}_{T}$ partially cancels out. Many techniques exist to handle this situation including partially reconstructed masses which have poor sensitivity, or the collinear approximation where the neutrinos are assumed to point in the same direction as the lepton or hadron which only apply to a fraction of the events. ${ }^{1}$ The best algorithm available, the Missing Mass Calculator (MMC), was implemented for this analysis [30]. Rather than assume collinearity, the MMC uses the mass and decay kinematics of the tau to find mutual orientations of the neutrinos and other decay products. Computing the MMC involves solving the system of equations in Eq. 4.1 completely.

$$
\begin{gathered}
\mathbb{E}_{T_{x}}=p_{m i s 1} \sin \theta_{m i s 1} \cos \phi_{m i s 1}+p_{m i s 2} \sin \theta_{m i s 2} \cos \phi_{m i s 2} \\
\mathscr{E}_{T_{y}}=p_{m i s 1} \sin \theta_{m i s 1} \sin \phi_{m i s 1}+p_{m i s 2} \sin \theta_{m i s 2} \sin \phi_{m i s 2} \\
M_{\tau 1}^{2}=m_{m i s 1}^{2}+m_{v i s 1}^{2}+2 \sqrt{p_{v i s 1}^{2}+m_{v i s 1}^{2}} \sqrt{p_{m i s 1}^{2}+m_{m i s 1}^{2}}-2 p_{v i s 1} p_{m i s 1} \cos \Delta \theta_{\nu m 1} \\
M_{\tau 2}^{2}=m_{m i s 2}^{2}+m_{v i s 2}^{2}+2 \sqrt{p_{v i s 2}^{2}+m_{v i s 2}^{2}} \sqrt{p_{m i s 2}^{2}+m_{m i s 2}^{2}}-2 p_{v i s 2} p_{m i s 2} \cos \Delta \theta_{\nu m 1}
\end{gathered}
$$

$\mathbb{E}_{T_{x}}$ and $\mathscr{E}_{T_{x}}$ are the $x$ and $y$ components of $\overrightarrow{\mathbb{E}}_{T}$. The subscripts vis $1 / 2(m i s 1 / 2)$ on $m$, $p, \theta$, and $\phi$ correspond to the visible (missing) mass, momentum, polar and azimuthal angles of the decay products. $\Delta \theta_{\nu m}$ is the angle between $\vec{p}_{m i s}$ and $\vec{p}_{v i s}$, and $M_{\tau}=1.777$ $\mathrm{GeV}$. The case relative to this analysis involves one leptonically (subscript 1) decaying tau and one hadronically (subscript 2 ) decaying tau. In this case, $m_{m i s 2}=0$ since there is only one neutrino, leaving seven unknowns $\left(p_{m i s 1 / 2}, \theta_{m i s 1 / 2}, \phi_{m i s 1 / 2}, m_{m i s 1}\right)$ and only four equations. It is necessary then to perform a scan in the three dimensional phase space of $\left(\phi_{m i s 1}, \phi_{m i s 2}, m_{m i s 1}\right)$. This generates many solutions, some physical the remaining non-physical. The invariant mass is constructed from the physical solutions, but it is possible to determine which solutions are more probable based on kinematic considerations such as $\Delta R=\sqrt{\left(\eta_{v i s}-\eta_{m i s}\right)^{2}+\left(\phi_{v i s}-\phi_{m i s}\right)^{2}}$.

\footnotetext{
${ }^{1}$ The collinear approximation was used in previous versions of this analysis.
} 
This variable is used to create a global event probability which provides an additional constraint and improves the di-tau invariant mass resolution.

Another added advantage of this technique is that the distribution of the number of physical solutions in any single event can be a powerful discriminate in the multivariate analysis discussed in Chapter 5.

\subsubsection{Jets Selection}

Each event must contain two or more jets. This significantly lowers the statistics, but greatly increases the signal significance, and improves the overall sensitivity. In what follows $X^{(1) /(2)}$ refers to the highest or second-highest $p_{T}$ jet.

- $p_{T}^{(1)}>25(20) \mathrm{GeV}$ for Run IIa (IIb), all other jets must have $p_{T}>15 \mathrm{GeV}$. All jets must satisfy $|\eta|<3.4$.

- Vertex confirmation is required for jets from Run IIb due to the increased instantaneous luminosity. To be vertex confirmed, a jet must have at least two tracks pointing to the primary vertex.

The jet $p_{T}$ requirement ensures quality modeling of low $p_{T}$ jets as jets with $p_{T}<15$ $\mathrm{GeV}$ are not reliably reconstructed. The $p_{T}^{(2)}$ requirement is lower to increase the overall jet acceptance. Allowing only jets with $|\eta|<3.4$ ensures that the jets are relatively central, and reconstructed in an optimal region of the detector.

\subsubsection{Missing Energy Selection}

In a previous version of this analysis [29], it was necessary to make a selection on the missing energy significance, $\mathcal{S}$, in order to reduce the large background from MJ events (Section 4.5), and $Z \rightarrow e e . \mathbb{E}_{T}$ significance is defined in Section 3.4, and has low values for objects with $\mathbb{E}_{T}$ from detector mismeasurement, and higher values for objects with physical $\mathbb{E}_{T}$ i.e. real neutrinos. The $\mathbb{E}_{T}$ found in MJ or $Z \rightarrow e e$ 
events is entirely from detector effects, whereas the $\mathbb{E}_{T}$ from $Z \rightarrow \tau \tau$ or $W+$ jets is from actual neutrinos.

The large contribution of MJ events made the modeling of several important variables, like $p_{T}^{e}$ and $p_{T}^{(1)}$ difficult. By making a selection on $\mathcal{S}$ the modeling and signal significance are both improved. Two different selections were chosen, $\mathcal{S}>2.0$ for Run IIb and $\mathcal{S}>3.0$ for Run IIa. Figure 4.9 compares $\mathcal{S}$ before and after the selection. The Run IIa selection is higher because of the relative increase in MJ events, and it is also important to note that the MJ contribution in Run IIb is reduced to 0 with this selection (see Section 4.5). This selection reduces $H \rightarrow \tau \tau$ signals by about $26 \%$, the $H \rightarrow W W$ signals by about $40 \%$, and the $Z \rightarrow e e$ background by about $90 \%$.

\subsection{Multi-jet Background Estimation}

A potentially large background to this analysis comes from multi-jet (MJ) events. MJ events happen when jets are reconstructed as taus and electrons. This background contribution is estimated from data in two stages: (1) determine the shape of the MJ background using a "MJ enriched" region that is designed to contain mostly MJ events, and (2) normalize that shape to the number of MJ events for the signal region.

The MJ enriched region provides a $98 \%$ pure MJ sample that is orthogonal to the signal sample. It is constructed by requiring $0.3<\mathrm{NN}_{\tau}<0.9$, and $\mathcal{L}_{e}<0.85$, referred to as a "bad tau" and "bad electron" respectively. The lower bound of this region of $\mathrm{NN}_{\tau}$ was chosen to be the same as the tau certification lower limit; this region contains few real taus. The value of $\mathcal{L}_{e}=0.85$ was previously used by $\mathrm{D} \varnothing$ as the value above which only "good" quality electrons existed. Below this value of 


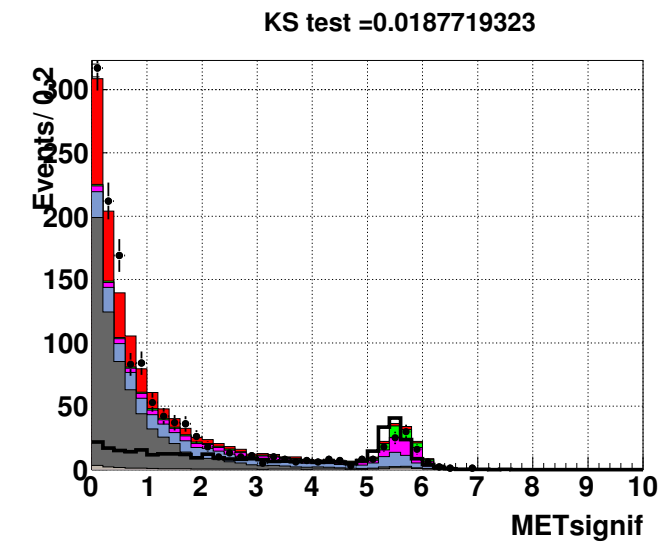

KS test $=0.972561611$

(a)

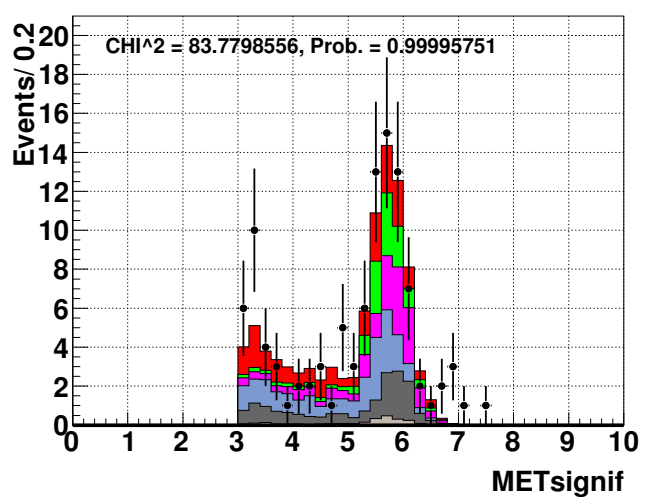

(b)

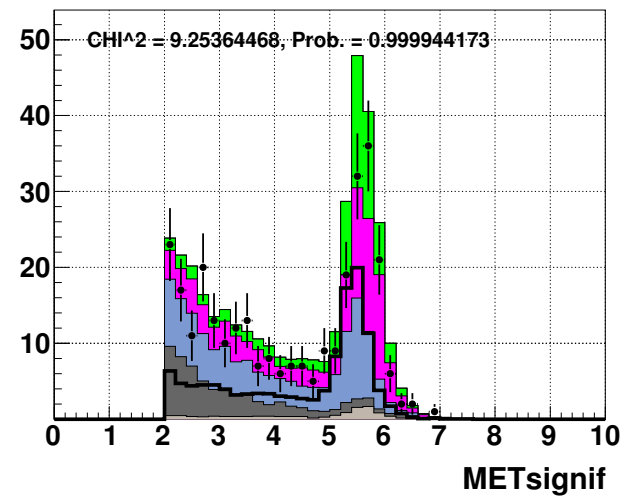

(c)

Figure 4.9. MET significance distributions (a) without the imposition of the $\mathcal{S}$ selection for Run IIa and Run IIb combined, and with the $\mathcal{S}$ selection for (b) Run IIa and (c) Run IIb. Missing energy actually resulting from neutrinos peaks around 6 , while missing energy from detector mismeasurement peaks at 0 .

$\mathcal{L}_{e}$ objects are very likely to be jets reconstructed as electrons. All other selections remain the same, with the exception of the selection on $\mathcal{S}$, which is removed to allow more MJ contribution thereby gaining statistics.

In the following $N$ represents the number of events in the signal sample, and $M$ represents the number of events in the MJ enriched sample. In both samples events are subdivided by the charge of the electron and the tau into same-sign (SS) and 
opposite-sign (OS) events. The number of MJ events in the signal sample, $N_{\mathrm{OS}}^{\mathrm{MJ}}$, is calculated from:

$$
N_{\mathrm{OS}}^{\mathrm{MJ}}=\rho\left(N_{\mathrm{SS}}^{\mathrm{data}}-N_{\mathrm{SS}}^{\mathrm{SM}}\right)
$$

where

$$
\rho=\frac{\left(M_{\mathrm{OS}}^{\mathrm{data}}-M_{\mathrm{OS}}^{\mathrm{SM}}\right)}{\left(M_{\mathrm{SS}}^{\mathrm{data}}-M_{\mathrm{SS}}^{\mathrm{SM}}\right)}
$$

The shape of the MJ background is taken from the MJ enriched sample, and normalized by Eq. 4.2. The scale factor $\rho$ is derived for each tau type in the MJ enriched sample and is the ratio of OS to SS events after residual SM backgrounds are subtracted. $^{2} \rho$ accounts for any differences of the ratio of OS to SS events between the MJ enriched sample and the signal sample. Table 4.1 summarizes the events, purity and $\rho$ factors for Run IIa.

It is critical to check the $\rho$ factors dependence on kinematic quantities. If the ratio of OS to SS events in the MJ enriched sample have a kinematic dependence, it would alter the shape of the MJ derived from the signal sample. Figures 4.10 and 4.11 show that $\rho$ is flat with $p_{T}$ or $\eta$ of the electron, tau and leading jet. The red line is a flat line fit to data in the region with sufficient statistics to make a reliable fit.

For the Run IIb data $N_{\mathrm{OS}}^{\mathrm{MJ}}=-1.2$ out of 296 events, or a contribution of essentially $0 \%$ MJ events and in future calculations the MJ was therefore scaled to 0. For Run IIa $N_{\mathrm{OS}}^{\mathrm{MJ}}=19.4$ out of 104 events, or about $20 \%$ MJ contribution. The strong rejection of MJ in Run IIb is largely due to the selection made on $\mathcal{S}$. The electron multivariate identification, $\xi_{e}$ also helps reject large amounts of MJ as compared to the previous version of this analysis.

\footnotetext{
${ }^{2}$ This subtraction is a very small effect as the MJ enriched purity is roughy $98 \%$ MJ events.
} 
Table 4.1. Number of events, purity (fraction of events estimated to be MJ) and scale factors, by tau type for the Run IIa MJ enriched sample.

\begin{tabular}{c|c|c|c|c}
\hline \hline Sample & & $\tau$ type 1 & $\tau$ type 2 & $\tau$ type 3 \\
\hline MJ enriched & $\mathrm{N}_{\text {evnts }}$ & 81 & 523 & 1016 \\
& purity & 0.963 & 0.981 & 0.980 \\
& $\rho$ & $1.314 \pm 0.295$ & $1.245 \pm 0.110$ & $1.087 \pm 0.074$ \\
\hline \hline
\end{tabular}

\subsection{Event Yields}

To better understand the effects of the selections made in the previous section, cut flows are developed in Tables 4.2 and 4.3. Preselection contains all the selections listed in Section 4.4 except for those listed in the table. The two or more jet requirement drastically reduces both the total background and the amount of expected signal, however it makes modeling of kinematic variables easier. The number of MJ events is not accurately calculated until the implementation of the OS $\tau / e$ selection, and hence the significance $\left(\frac{S}{\sqrt{B}} \cdot 1000\right)$ is not accurately known. The type 1 ICR selection reduces the number of $Z \rightarrow e e$ by roughly a factor of three, while the selection on $\mathbb{E}_{T}$ significance greatly reduces both the MJ and $Z \rightarrow$ ee backgrounds.

Table 4.2. Events retained after successive cuts for the Run IIa portion of the analysis, for data and the expected SM and MJ backgrounds. The signal yields are quoted for $m_{H}=125 \mathrm{GeV}$.

\begin{tabular}{c|cccccccccc}
\hline \hline cut & $t \bar{t}$ & $W+j$ & $Z_{e e}+j$ & $Z_{\tau \tau}+j$ & DB & MJ & Ebkgd & Data & Higgs & Signif \\
\hline preselect & 28.9 & 1150 & 14693 & 2282 & 55.9 & 5778 & 23989 & 28989 & 2.47 & - \\
2 jet & 22.6 & 40.1 & 370.6 & 62.1 & 9.1 & 382 & 886 & 1295 & 0.56 & - \\
OS $\tau / e$ & 19.6 & 24.9 & 349.6 & 58.6 & 7.9 & 382 & 842 & 893 & 0.49 & 16.8 \\
$\tau_{1}$ ICR & 16.6 & 23.4 & 124.6 & 54.5 & 4.7 & 337 & 561 & 554 & 0.44 & 18.6 \\
METsig & 12.9 & 16.3 & 14.6 & 20.9 & 2.3 & 21.9 & 88.9 & 104 & 0.26 & 28.6 \\
\hline \hline
\end{tabular}



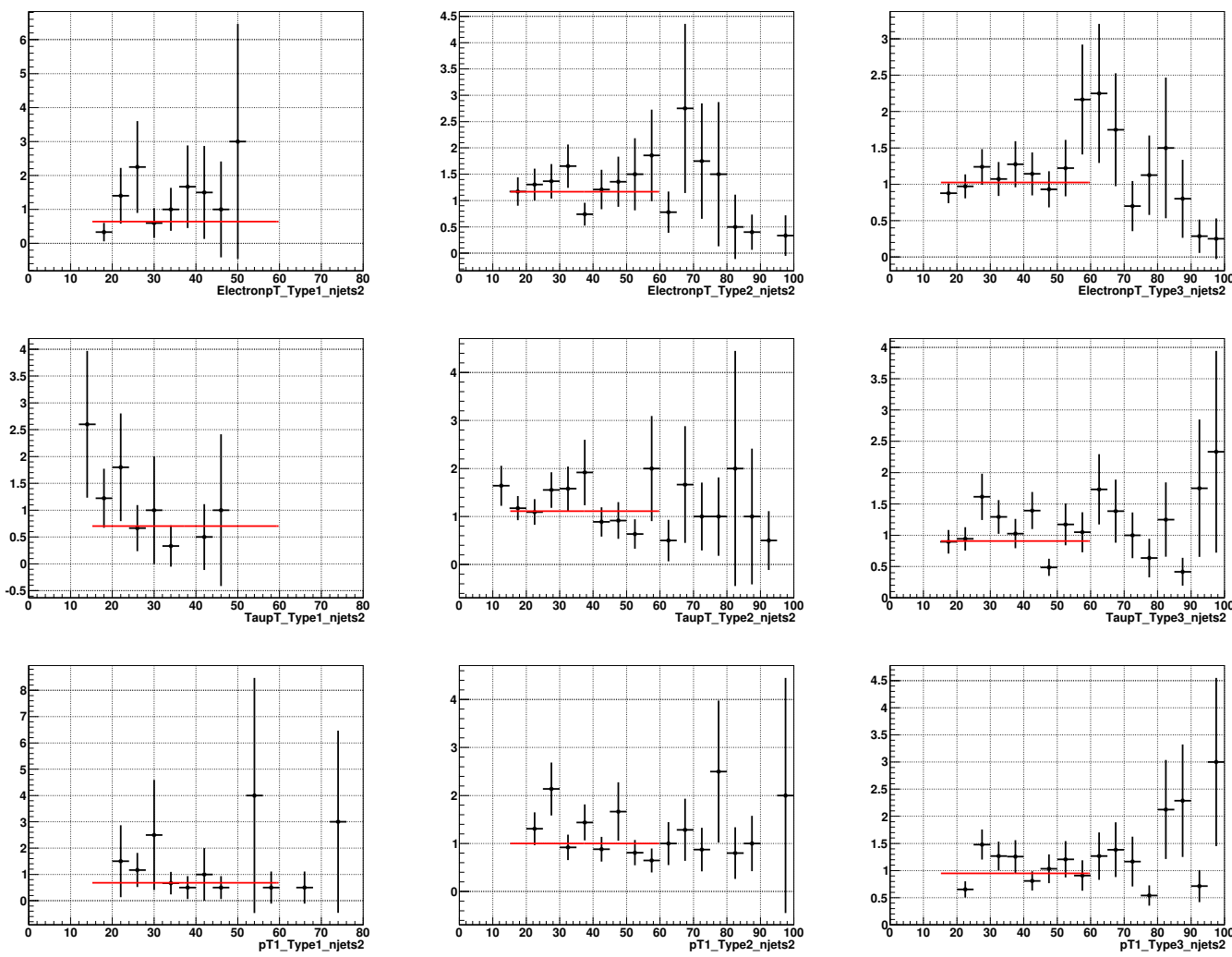

Figure 4.10. Distributions of the ratio of OS to SS events in the MJ enriched sample, as function of (top) $p_{T}^{e}$, (middle) $p_{T}^{\tau}$ and (bottom) $p_{T}^{(1)}$ for different $\tau$ types. The red line shows a flat fit and therefore no $\rho$ dependence within the good statistics region of each distribution.

Table 4.4 shows the contribution of each background by tau type for the full Run II final selection. The uncertainties on the backgrounds are discussed in Section 6.1. With the selection on $\mathcal{S}$, the dominant background becomes $Z \rightarrow \tau \tau$, which closely follows the kinematics of Higgs signal, so it is difficult to reduce via simple selection based methods.

Tables 4.5 and 4.6 show the contribution of the nine signal processes to the total amount of expected signal for two orthogonal enriched samples. Subsample T, is enriched with $H \rightarrow \tau \tau$ signals, and subsample $\mathrm{W}$ is enriched with $H \rightarrow W W$ signals. The reasoning behind and the procedure used to develop these subsamples is 

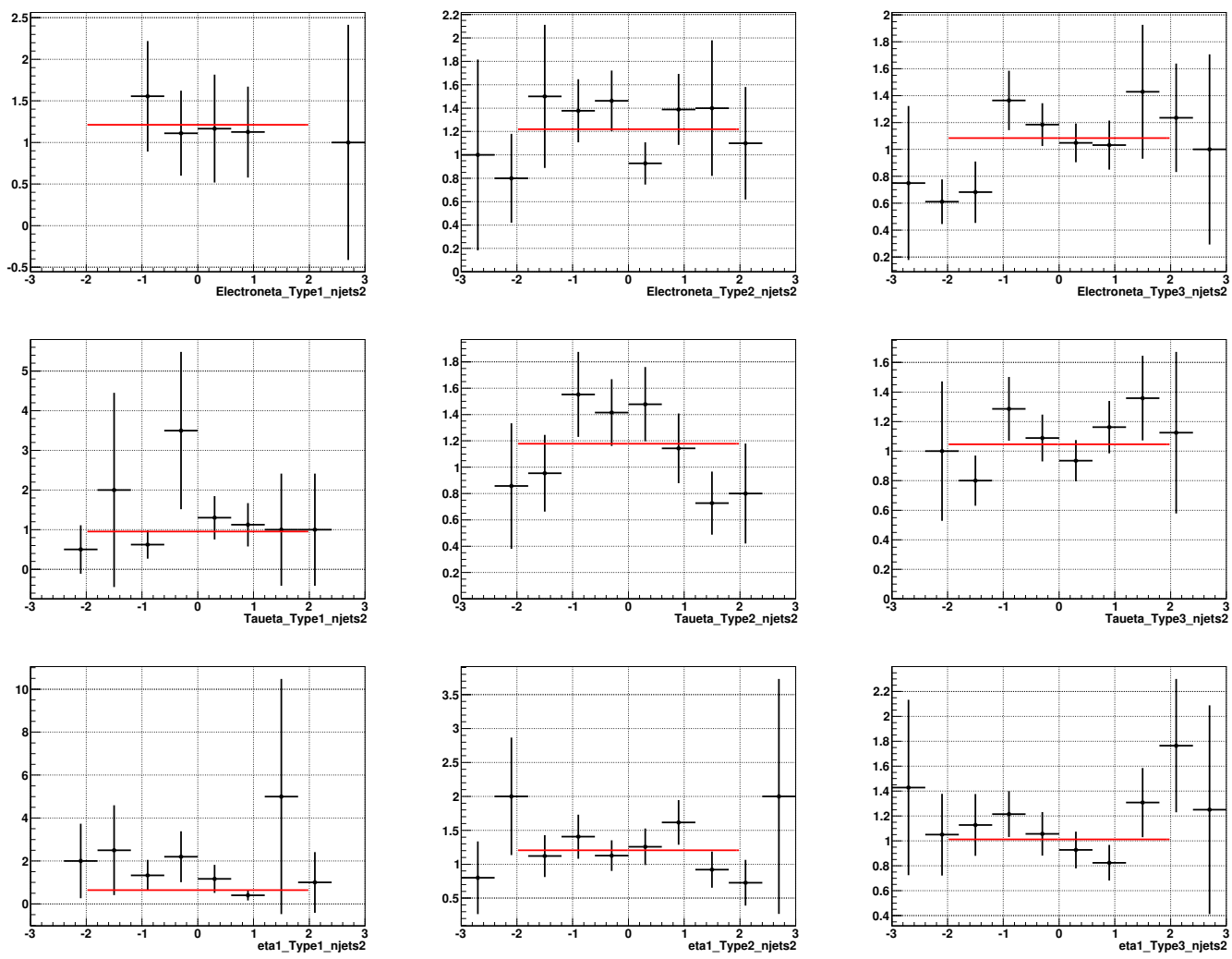

Figure 4.11. Distributions of the ratio of OS to SS events in the MJ enriched sample, as function of (top) $\eta_{e}$, (middle) $\eta_{\tau}$ and (bottom) $\eta^{(1)}$ for different $\tau$ types. The red line shows a flat fit and therefore no $\rho$ dependence within the good statistics region of each distribution.

discussed in Section 5.3. As expected from the Higgs branching ratio, the $\tau \tau$ signals dominate for lower masses, while the $W W$ signals play a larger role above $m_{H}=130$ $\mathrm{GeV}$.

\subsubsection{New $\mathrm{NN}_{\tau}$ and Electron MVA}

Two significant improvements made to the event selection were the use of the new $\mathrm{NN}_{\tau}$ and the electron multivariate, $\xi_{e}$. Both processes greatly improve the background rejection thus improving the significance of this search. When these object selections are combined with the event selection criteria previously mentioned the 
Table 4.3. Events retained after successive cuts for the Run IIb portion of the analysis, for data and the expected SM and MJ backgrounds. The signal yields are quoted for $m_{H}=125 \mathrm{GeV}$.

\begin{tabular}{c|cccccccccc}
\hline \hline cut & $t \bar{t}$ & $W+j$ & $Z_{e e}+j$ & $Z_{\tau \tau}+j$ & DB & MJ & Nbkgd & Data & Higgs & Signif. \\
\hline preselect & 181.2 & 7863 & 84483 & 11734 & 328.3 & 16110 & 120700 & 126431 & 12.6 & - \\
2 jet & 134.1 & 267.6 & 1776 & 295.1 & 44.3 & 916 & 3433 & 3828 & 2.41 & - \\
OS $\tau / e$ & 111.1 & 156.9 & 1654 & 273.6 & 37.9 & 916 & 3150 & 2711 & 2.05 & 36.5 \\
$\tau_{1}$ ICR & 95.9 & 147.3 & 640.0 & 255.3 & 22.8 & 846 & 2007 & 1754 & 1.83 & 40.8 \\
METsig & 76.5 & 100.4 & 54.5 & 109.1 & 10.6 & 0.0 & 351.0 & 296 & 1.16 & 63.5 \\
\hline \hline
\end{tabular}

Table 4.4. After preselection in the Run IIa and IIb combined, the number of data and the expected SM and MJ backgrounds for both the total and individual tau types. The signal yields are quoted for $m_{H}=125 \mathrm{GeV}$.

\begin{tabular}{c|cccccccccc}
\hline \hline$\tau$ type & $t \bar{t}$ & $W+j$ & $Z_{e e}+j$ & $Z_{\tau \tau}+j$ & DB & MJ & sbkgd & Data & Higgs & Signif. \\
\hline type 1 & 4.5 & 4.6 & 0.07 & 9.8 & 0.9 & 2.0 & $23.0 \pm 1.8$ & 15 & 0.10 & 20.1 \\
type 2 & 57.7 & 64.9 & 66.6 & 91.7 & 8.3 & 10.1 & $290.8 \pm 21.0$ & 261 & 1.03 & 60.5 \\
type 3 & 27.2 & 47.2 & 2.4 & 28.5 & 3.7 & 9.8 & $123.7 \pm 9.6$ & 124 & 0.33 & 29.7 \\
\hline All & 89.4 & 116.7 & 69.1 & 130.0 & 12.9 & 21.9 & $437.5 \pm 23.2$ & 400 & 1.46 & 69.8 \\
\hline \hline
\end{tabular}

Table 4.5. Number of events for each signal/decay channel expected in the final selection in the $\mathrm{T}$ subsample as a function of Higgs boson mass.

\begin{tabular}{c|ccccc|cccc|c}
\hline \hline$m_{H}$ & $\mathrm{HZ}$ & $\mathrm{ZH}_{\tau \tau}$ & $\mathrm{WH}_{\tau \tau}$ & $\mathrm{GGF}_{\tau \tau}$ & $\mathrm{VBF}_{\tau \tau}$ & $\mathrm{ZH}_{W W}$ & $\mathrm{WH}_{W W}$ & $\mathrm{GGF}_{W W}$ & $\mathrm{VBF}_{W W}$ & Total \\
\hline 105 & 0.111 & 0.295 & 0.462 & 0.250 & 0.227 & 0.004 & 0.002 & 0.004 & 0.001 & 1.358 \\
110 & 0.100 & 0.266 & 0.427 & 0.285 & 0.235 & 0.007 & 0.004 & 0.003 & 0.002 & 1.328 \\
115 & 0.085 & 0.257 & 0.405 & 0.276 & 0.208 & 0.017 & 0.011 & 0.002 & 0.003 & 1.265 \\
120 & 0.074 & 0.220 & 0.336 & 0.250 & 0.184 & 0.019 & 0.019 & 0.004 & 0.004 & 1.111 \\
125 & 0.060 & 0.188 & 0.277 & 0.241 & 0.182 & 0.024 & 0.021 & 0.005 & 0.017 & 1.018 \\
130 & 0.043 & 0.159 & 0.232 & 0.197 & 0.149 & 0.045 & 0.032 & 0.009 & 0.010 & 0.877 \\
135 & 0.036 & 0.127 & 0.176 & 0.169 & 0.127 & 0.054 & 0.050 & 0.012 & 0.030 & 0.780 \\
140 & 0.023 & 0.089 & 0.132 & 0.129 & 0.096 & 0.054 & 0.043 & 0.014 & 0.015 & 0.595 \\
145 & 0.018 & 0.060 & 0.087 & 0.098 & 0.070 & 0.072 & 0.050 & 0.013 & 0.015 & 0.484 \\
150 & 0.011 & 0.041 & 0.058 & 0.061 & 0.046 & 0.051 & 0.063 & 0.014 & 0.014 & 0.358 \\
\hline \hline
\end{tabular}


Table 4.6. Number of events for each signal/decay channel expected in the final selection in the $\mathrm{W}$ subsample as a function of Higgs boson mass.

\begin{tabular}{c|ccccc|cccc|c}
\hline \hline$m_{H}$ & $\mathrm{HZ}$ & $\mathrm{ZH}_{\tau \tau}$ & $\mathrm{WH}_{\tau \tau}$ & $\mathrm{GGF}_{\tau \tau}$ & $\mathrm{VBF}_{\tau \tau}$ & $\mathrm{ZH}_{W W}$ & $\mathrm{WH}_{W W}$ & $\mathrm{GGF}_{W W}$ & $\mathrm{VBF}_{W W}$ & Total \\
\hline 105 & 0.024 & 0.049 & 0.068 & 0.071 & 0.032 & 0.007 & 0.012 & 0.012 & 0.002 & 0.277 \\
110 & 0.021 & 0.050 & 0.066 & 0.056 & 0.032 & 0.012 & 0.026 & 0.015 & 0.005 & 0.283 \\
115 & 0.021 & 0.053 & 0.060 & 0.043 & 0.035 & 0.025 & 0.039 & 0.012 & 0.010 & 0.300 \\
120 & 0.017 & 0.041 & 0.064 & 0.057 & 0.033 & 0.045 & 0.077 & 0.027 & 0.016 & 0.376 \\
125 & 0.013 & 0.036 & 0.057 & 0.057 & 0.026 & 0.070 & 0.118 & 0.037 & 0.027 & 0.442 \\
130 & 0.017 & 0.031 & 0.032 & 0.037 & 0.032 & 0.086 & 0.184 & 0.072 & 0.044 & 0.536 \\
135 & 0.010 & 0.022 & 0.034 & 0.038 & 0.020 & 0.111 & 0.214 & 0.100 & 0.052 & 0.601 \\
140 & 0.008 & 0.015 & 0.019 & 0.027 & 0.015 & 0.161 & 0.260 & 0.120 & 0.076 & 0.703 \\
145 & 0.004 & 0.011 & 0.018 & 0.021 & 0.013 & 0.175 & 0.296 & 0.145 & 0.094 & 0.778 \\
150 & 0.003 & 0.007 & 0.009 & 0.015 & 0.008 & 0.224 & 0.385 & 0.180 & 0.120 & 0.953 \\
\hline \hline
\end{tabular}

Table 4.7. Comparison of the contributions from each background by percent for the previous and current results.

\begin{tabular}{c|c|c}
\hline \hline \multicolumn{3}{c}{ Background Contributions by $\%$} \\
\hline Sample & $4.3 \mathrm{fb}^{-1}$ & $9.7 \mathrm{fb}^{-1}$ \\
\hline$Z_{e e}+j$ & $9 \%$ & $16 \%$ \\
$Z_{\tau \tau}+j$ & $26 \%$ & $30 \%$ \\
$W+j$ & $23 \%$ & $27 \%$ \\
$t \bar{t}$ & $13 \%$ & $20 \%$ \\
DB & $2 \%$ & $2 \%$ \\
MJ & $27 \%$ & $5 \%$ \\
\hline Signif. & 27.1 & 69.8 \\
\hline \hline
\end{tabular}

proportion of backgrounds is altered as compared to the previous result. Table 4.7 shows the percent contribution of each background for the current and previous result. The proportional increase of the $Z \rightarrow \tau \tau$ background is helpful because the majority of signals have kinematic properties similar to this background. The large increase in significance reflects the increased signal acceptance, and background rejection. 


\section{CHAPTER 5}

\section{MULTIVARIATE ANALYSIS}

Searches from ATLAS [12] and CMS [13] look for excess events in a well modeled variable such as the invariant mass of the $\tau \tau$ system. Compared to searches at the LHC, Tevatron searches have lower center of mass energy and less luminosity; to recover sensitivity from these differences multivariate analyses (MVA) are employed. For optimal sensitivity it is possible to create a final variable that best separates signal from background and look for excess events in the signal region of that distribution.

Gradient Boosted Decision Trees, or BDTs, are used in this analysis. In each decision tree the sample is split into signal-like and background-like samples using one of the input variables (Section 5.1). The decision tree minimizes the Gini index $G=p(1-p)$, where $p$ is the purity for signal-like events, $p=S /(S+B)$, and $(1-p)$ is the purity for background events. A purity of 0 or 1 is equally desirable as it helps classify the event as likely signal or likely background. The decision tree then splits the daughter samples, or nodes, further by selecting on another variable by again minimizing the Gini index. After reaching the maximum number of allowed nodes, or until no further distinction can be made the splitting stops and each sample, called a leaf, is weighted to a value between -1 and 1 according to how signal-like $(+1)$ or background-like (-1) it is. Figure 5.1 shows a sample decision tree with four levels.

Boosted decision trees differ from simple decision trees in that several iterations of the aforementioned procedure are used. After each BDT, events are compared to the known signal-background distribution, and events which are misclassified are given 


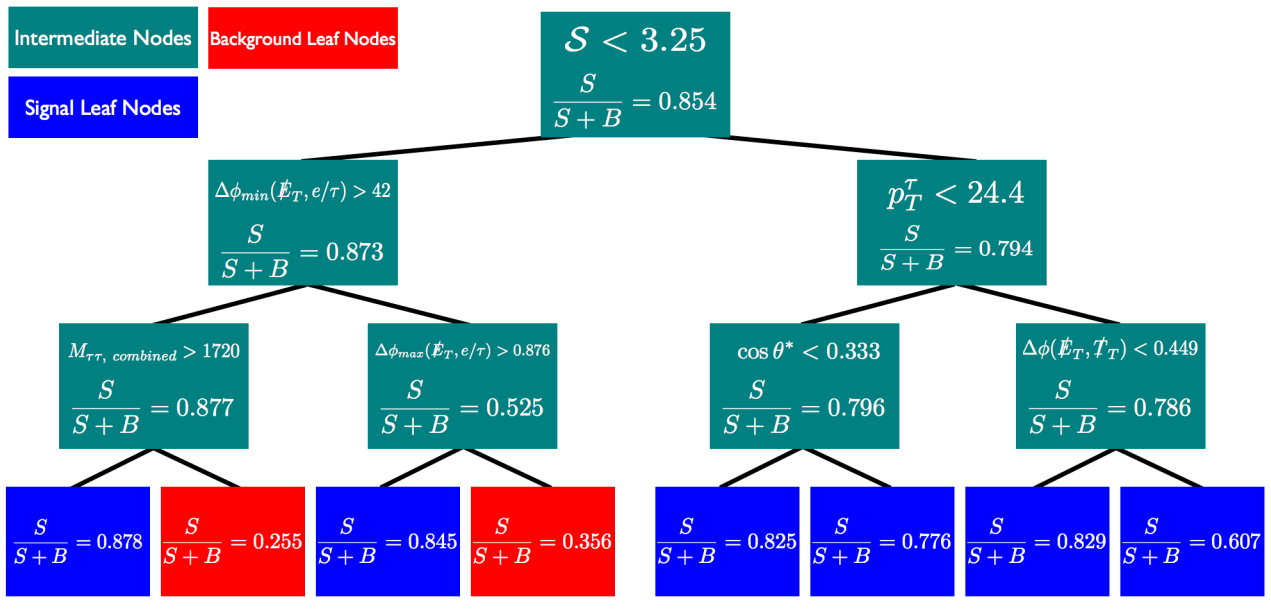

Figure 5.1. An example decision tree showing the splitting of the sample into signallike and background-like nodes.

a weight which makes it more difficult for these events to be misclassified in the next BDT iteration.

A number of parameters are used to control the BDT process, which are all nominal for this analysis. The signal and background samples used for training have much further reaching effects than any of the BDT parameters (See Section 5.4 - 5.5) $N_{\text {trees }}=400$ is the number of individual decisions trees that are used, $N_{\text {node }}^{\max }=15$ limits the number of nodes in any single tree, shrinkage $=0.6$ reduces the effect of overtraining due to statistical fluctuations, and the bagging fraction $=0.6$ quantifies the amount of random sampling that each node populates its sample with in order to reduce the effect of statistical fluctuations.

\subsection{Input Variables}

32 variables are used in each multivariate analysis performed. There are standard kinematic variables such as $p_{T}$, invariant masses, and angular variables as well as some more sophisticated variables such as $\xi_{e}$, and the number of physical solutions 
(from the MMC). Some variables inclusion into this analysis are historical, but it was found that inclusion of several variables with poor discriminating power is helpful to the overall sensitivity. For instance, using five poorly discriminating variables adds as much power as one strongly discriminating variable. The details of these variables are given below.

1. $p_{T}^{e}$ : the transverse momentum of the electron candidate.

2. $p_{T}^{\tau}$ : the transverse momentum of the hadronic tau candidate.

3. $p_{T}^{(1)}$ : the transverse momentum of the leading jet candidate.

4. $p_{T}\left(e, \tau, \mathbb{E}_{T}\right)$ : the transverse momentum of the $e, \tau$ and $\mathbb{E}_{T}$.

5. $H_{T}$ : scalar sum of the $p_{T}$ of all jets with $p_{T}>15 \mathrm{GeV}$ and $|\eta|<3.4$.

6. $S_{T}$ : the scalar sum of the $p_{T}$ of the electron candidate, the tau candidate, the two candidate jets and of the event missing transverse energy, $S_{T}=p_{T}^{e}+p_{T}^{\tau}+$ $p_{T}^{(1)}+p_{T}^{(2)}+\mathscr{E}_{T}$.

7. $V_{T}$ : the magnitude of the vector sum of the $p_{T}$ 's of the electron candidate, the tau candidate, and the two candidate jets, $V_{T}=\left|\vec{p}_{T}^{e}+\vec{p}_{T}^{\tau}+\vec{p}_{T}^{(1)}+\vec{p}_{T}^{(2)}\right|$.

8. $H_{T} / H_{T}$ : the ratio of the missing $H_{T}$, defined as the magnitude of the vector sum of all jet $p_{T}$ 's, to the scalar sum of the $p_{T}$ 's of all jets.

9. $\Delta R_{j j}: \Delta R=\sqrt{(\Delta \phi)^{2}+(\Delta \eta)^{2}}$ is the distance in azimuth $\phi$ and pseudorapidity $\eta$ between the two leading jets.

10. $\Delta \phi\left(\mathbb{E}_{T}, T_{T}\right)$ : the azimuthal angle difference between $\mathbb{E}_{T}$ and the missing transverse momentum, $T_{T}$, calculated as the negative of the vector sum of the $p_{T}$ of all tracks with at least eight CFT hits and a DCA to the primary vertex of less than $2 \mathrm{~mm}$.

11. $\Delta \phi_{\min }\left(\mathbb{E}_{T}\right.$, jets $)$ : the minimum azimuthal angle difference between the $\mathbb{E}_{T}$ and any good jet. 
12. $\Delta \eta(j j)$ : the absolute value of the pseudorapidity difference between the two leading jets.

13. $\Delta \phi\left(e \tau, j^{(1)} j^{(2)}\right)$ : the azimuthal angle difference between the $e-\tau$ system and the two leading jets system.

14. $\Delta \phi_{\min }\left(\mathbb{E}_{T}, e / \tau\right)$ : the minimum azimuthal angle difference between the $\mathbb{E}_{T}$ and the electron or tau.

15. $\Delta \phi_{\max }\left(\mathbb{E}_{T}, e / \tau\right)$ : the maximum azimuthal angles difference between the $\mathbb{E}_{T}$ and the electron or tau.

16. $\Delta \phi_{\max }\left(\mathbb{E}_{T}, j^{(1)} / j^{(2)}\right)$ : the maximum azimuthal angle difference between the $\mathbb{E}_{T}$ and leading or subleading jet.

17. $\Delta \phi\left(e, j^{(1)}\right)$ : the azimuthal angle difference between the electron and the leading jet.

18. $\cos \theta^{*}: \theta^{*}$ is the angle between the leading plus subleading jet and the proton beam direction in the lab frame.

19. $M_{j j}$ : invariant mass of the two candidate jets. Invariant mass is calculate by

$$
M^{2}=m_{1}^{2}+m_{2}^{2}+2\left(E_{T 1} E_{T 2}-\vec{p}_{T 1} \cdot \vec{p}_{T 2}\right)
$$

20. $M_{T}^{e}$ : transverse mass calculated from the $p_{T}^{e}$ and $\mathbb{E}_{T}$. Transverse mass is calculated by

$$
M_{T}=\left(E_{T 1}+E_{T 2}\right)^{2}-\left(p_{x 1}+p_{x 2}\right)^{2}-\left(p_{y 1}+p_{y 2}\right)^{2}
$$

21. $M_{T}^{\tau}$ : transverse mass calculated from the $p_{T}^{\tau}$ and $\mathbb{E}_{T}$.

22. $M_{T}^{\min }\left(\mathbb{E}_{T}, e / \tau\right)$ : the minimum of transverse mass between the $\mathbb{E}_{T}$ and the electron of the tau.

23. $M_{T}\left(\mathbb{E}_{T}, e \tau\right)$ : the transverse mass between the $\mathbb{E}_{T}$ and the $e-\tau$ system.

24. $M\left(e, \tau, j_{1}, j_{2}\right)$ : the four-body invariant mass of the two leading jets, the electron and the tau candidates. 
25. $M_{\tau \tau}^{\text {combined }}$ : the invariant MMC $\tau \tau$ mass when there is a solution, and the $\tau \tau$ mass by collinear approximation when there is none.

26. $M_{W W}^{\text {combined }}$ : the invariant MMC $W W$ mass when there is solution, and $W W$ mass estimated as

$$
\frac{M_{T}\left(\mathscr{E}_{T}, e+\tau\right) \cdot \mathrm{p}(e+\tau)}{p_{T}(e+\tau)}
$$

when there is none. $\mathrm{p}(e+\tau)$ is the scalar some of those particle momenta. One $W$ boson is on-shell while the other is off-shell, and the MMC solutions vary depending on which $W$ is on-shell. Both possibilities are considered, each one is solved separately and then the two are averaged.

27. $N_{\tau \tau}^{\text {soln.: }}$ the number of physical $\tau \tau$ MMC solutions in the event.

28. $N_{W W}^{\text {soln. }}$ : the number of physical $W W$ MMC solutions in the event. The average was taken for the same reason in $M_{W W}^{\text {combined }}$.

29. $A\left(\mathbb{E}_{T}, H_{T}\right)$ : Asymmetry between $\mathbb{E}_{T}$ and $\mathbb{H}_{T},\left(\mathbb{E}_{T}-\not_{T}\right) /\left(\mathbb{E}_{T}+\mathbb{H}_{T}\right)$.

30. $\mathcal{S}$ : the missing $E_{T}$ significance.

31. $\xi_{e}$ : the electron multivariate output.

32. $m_{H}$ : the Higgs boson mass.

Variables 1-8 all relate to transverse momentum. Generally speaking, variables using jet $p_{T}$ 's are more reliable than those involving $p_{T}$ 's from electrons or taus, since these measure any recoil from that system which could be neutrinos from a background process such as $t \bar{t}$. Variables 9-18 all deal with angle differences. With the various signal processes included in this analysis the angular distributions can be quite different from one another. Including variables that can exploit even just one signal is advantageous in the MVA procedures.

Variables 19-26 are all reconstructed masses using various objects. If the mass of the Higgs is very different from the mass of the $W$ or the $Z$, looking for an excess in 
reconstructed mass plots would be relatively straight forward. Variables 27 and 28 are related to the MMC calculation. The shape of background and signal are particularly different for these two variables and therefore they are useful discriminants. Variable 29 , the asymmetry of $\mathbb{E}_{T}$ and $\mathscr{H}_{T}$, was introduced in the $H \rightarrow \nu \nu b b$ analysis and is sensitive to the difference in missing energy from the jet system to the whole $\mathbb{E}_{T}$. It helps reject soft objects present in $\mathbb{E}_{T}$ but not $\not_{T}$. Even after making selections on variables 30 and $31\left(\mathcal{S}\right.$ and $\left.\xi_{e}\right)$, they are both is still helpful in discriminating MJ and $Z \rightarrow e e$ backgrounds. Finally variable $32, m_{H}$, is used to help eliminate BDT fluctuations between mass points (see Section 5.3). Figures 5.2 - 5.8 show all the BDT input variables and several other key kinematic distributions. It is necessary to show good modeling between data and MC before proceeding any further. The stepped structure of Fig. 5.8 (d) is a consequence of having two different selections of $\mathcal{S}$ in Run IIa and IIb. 


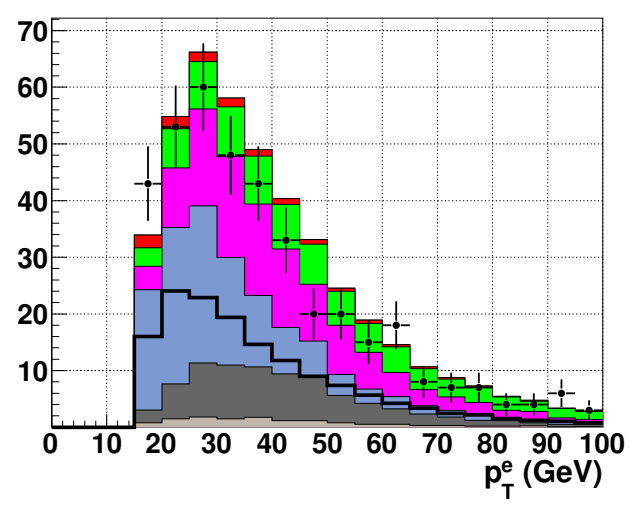

(a)

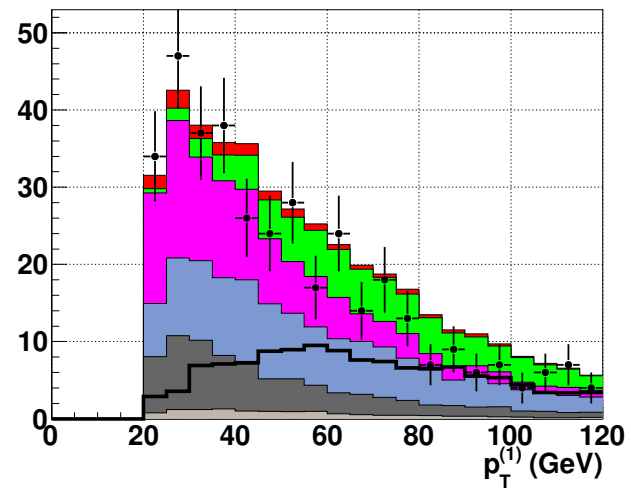

(c)

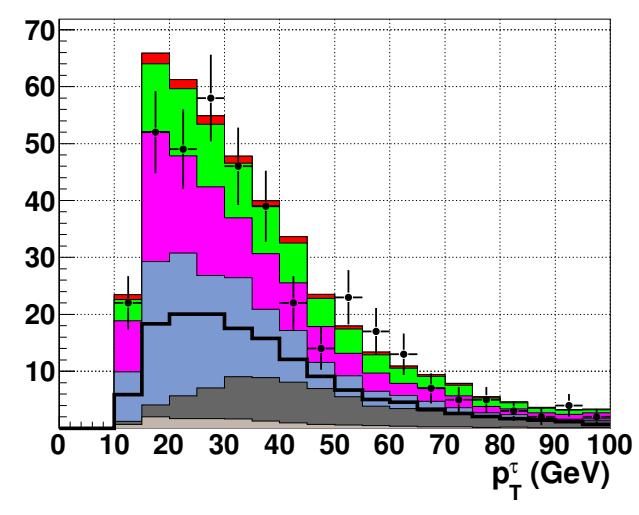

(b)

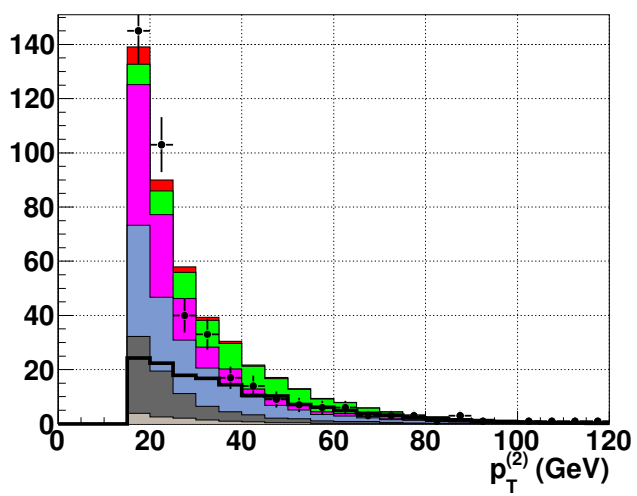

(d)

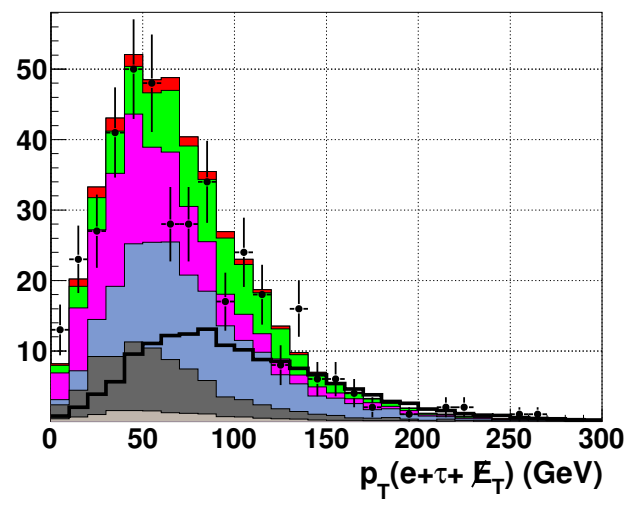

(e)

Figure 5.2. Transverse momentum distributions for various objects, and the combined $p_{T}$ of the tau, electron and $\mathbb{E}_{T}$. The signals are shown multiplied by 250. 


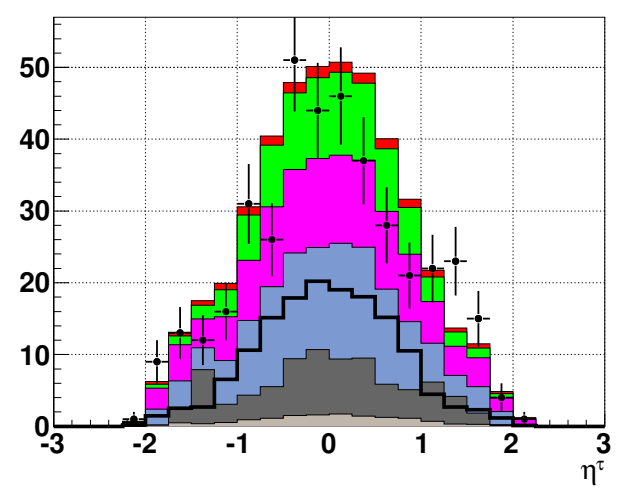

(a)

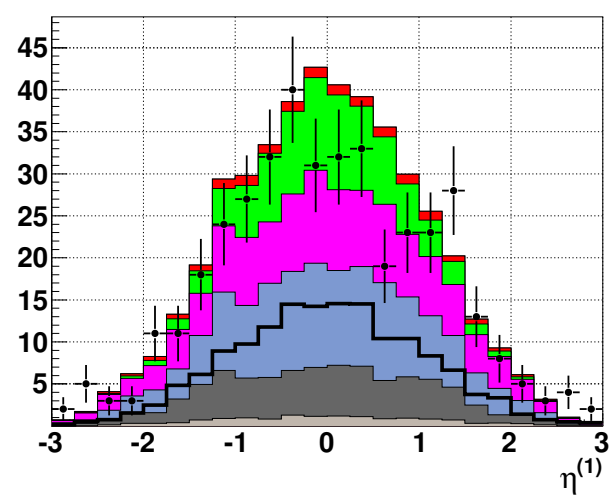

(c)

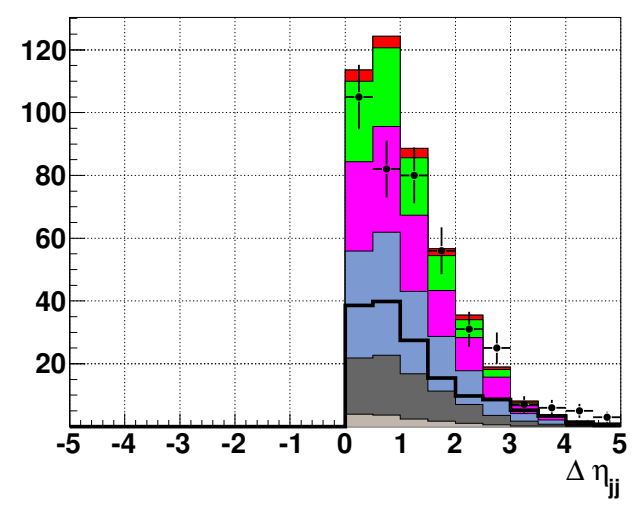

(e)

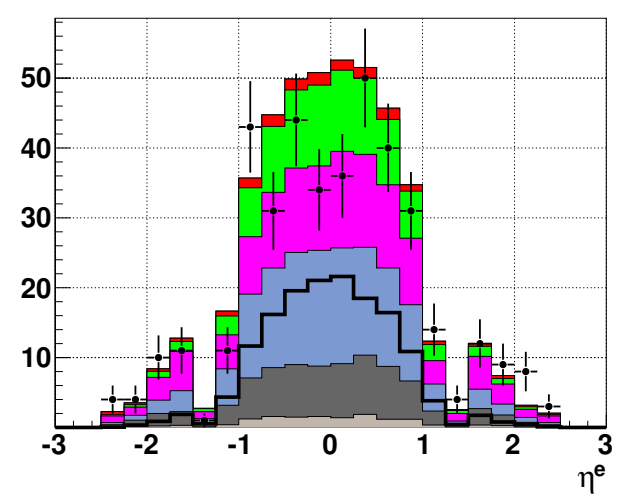

(b)

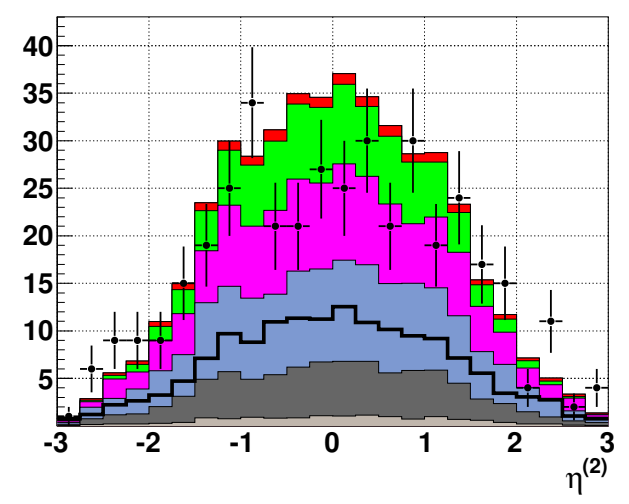

(d)

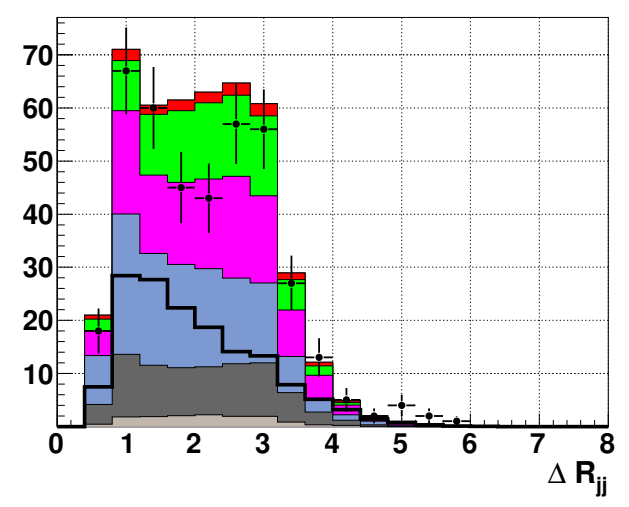

(f)

Figure 5.3. $\eta$ distributions for various objects, and $\Delta R$ between the jets. The signals are shown multiplied by 250 . 


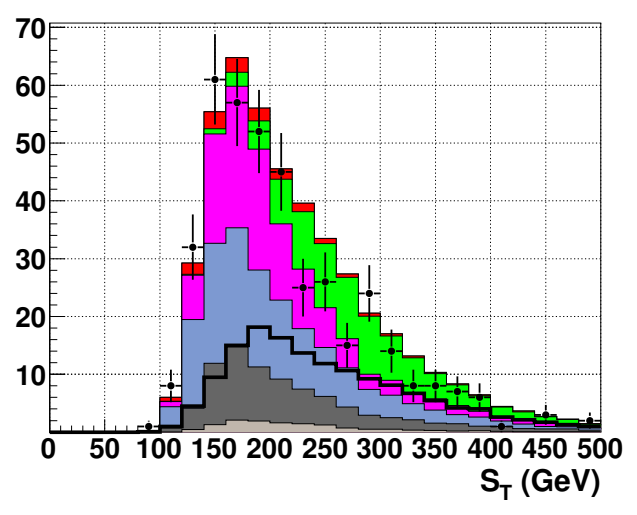

(a)

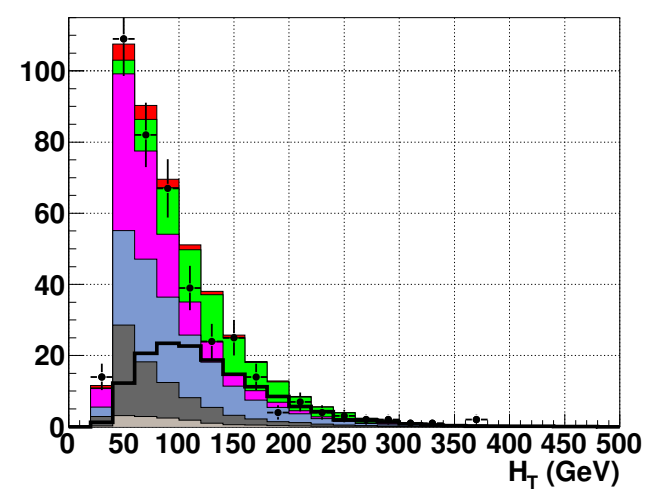

(c)

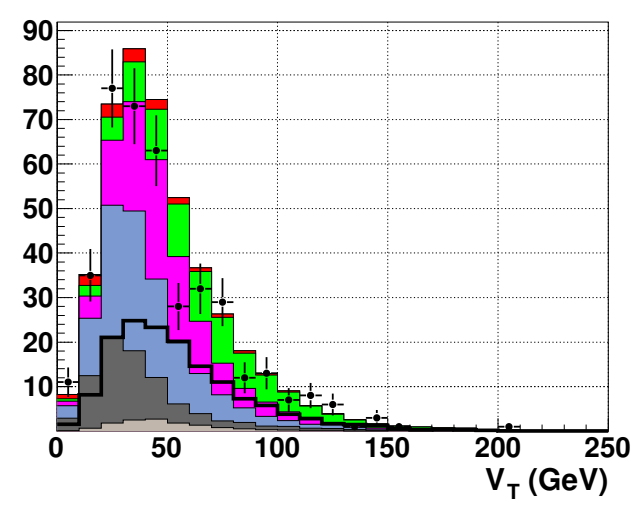

(b)

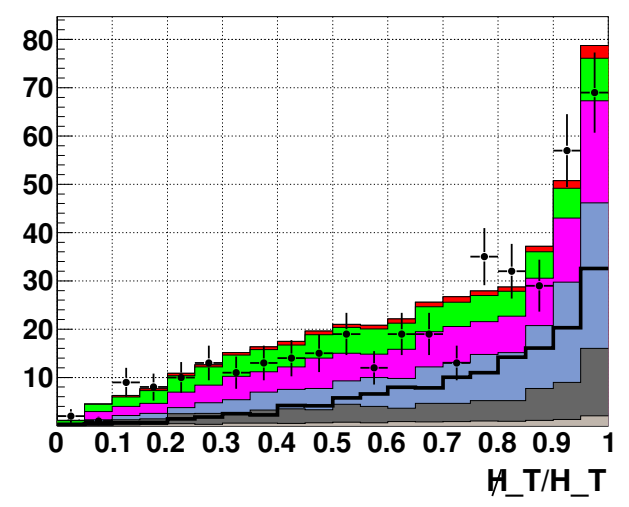

(d)

Figure 5.4. $p_{T}$ distributions for various object combinations. The signals are shown multiplied by 250 . 


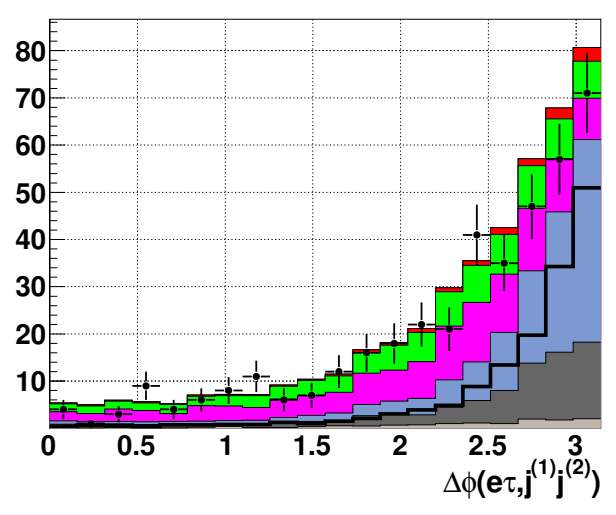

(a)

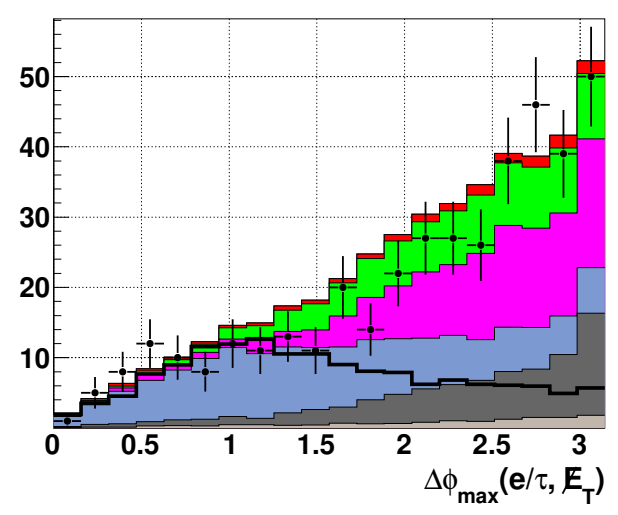

(c)

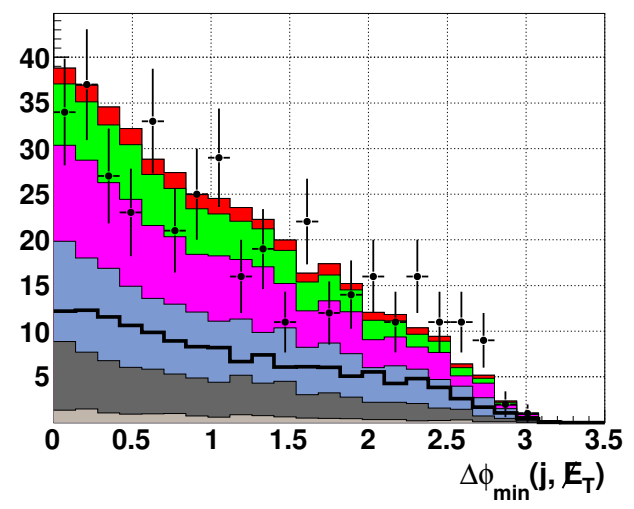

(e)

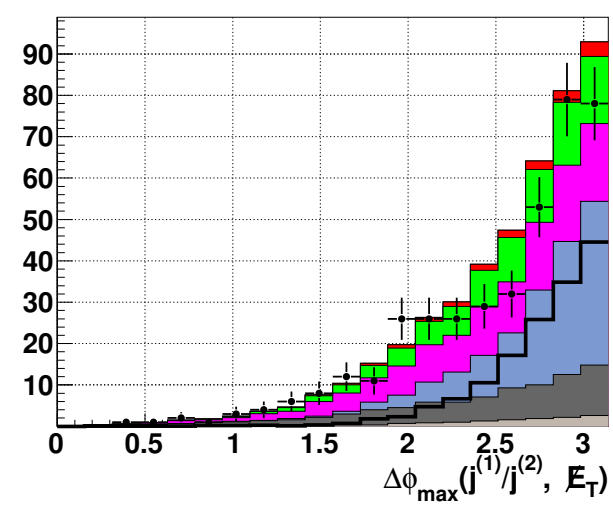

(b)

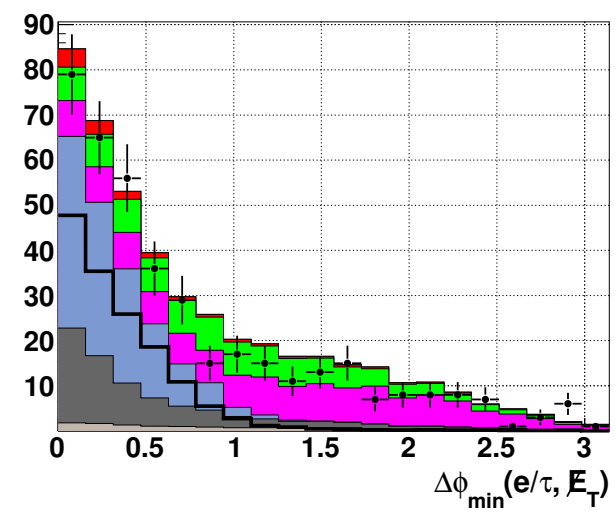

(d)

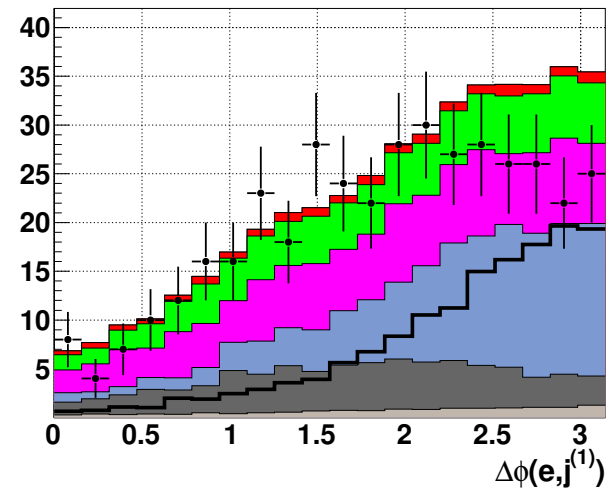

(f)

Figure 5.5. $\Delta \phi$ minimum and maximum distributions for various object combinations. The signals are shown multiplied by 250 . 


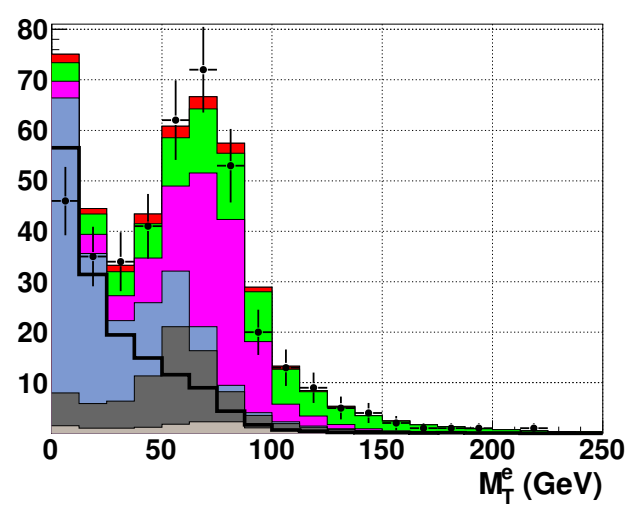

(a)

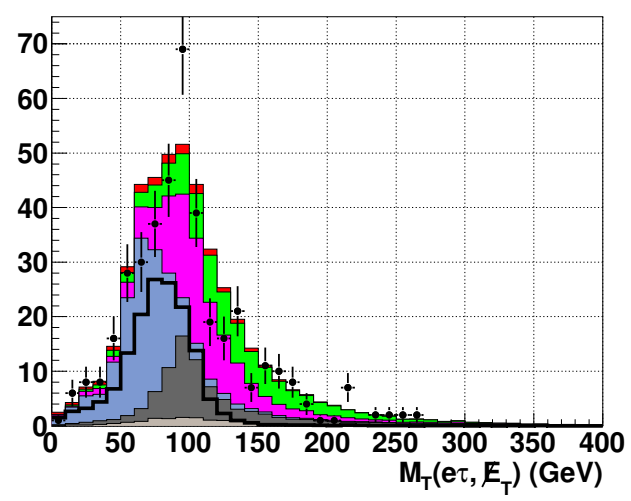

(c)

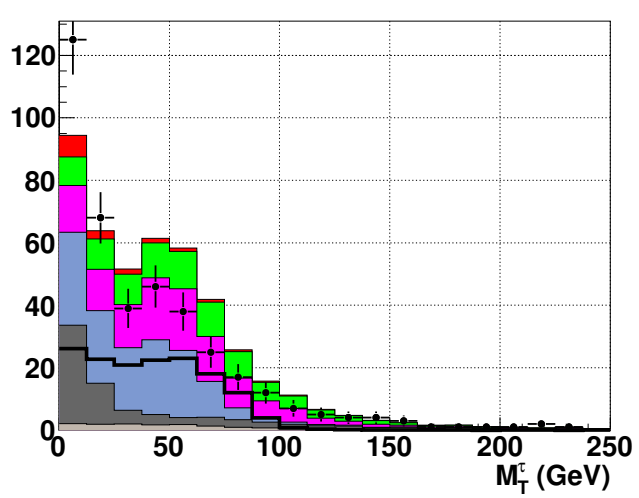

(b)

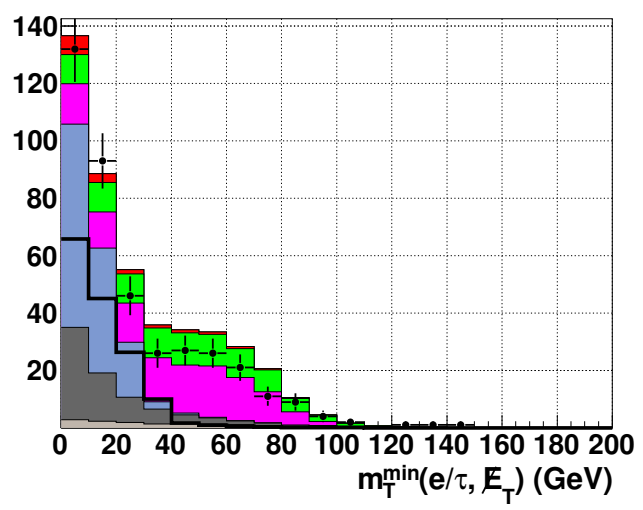

(d)

Figure 5.6. Transverse mass distributions. The signals are shown multiplied by 250 . 


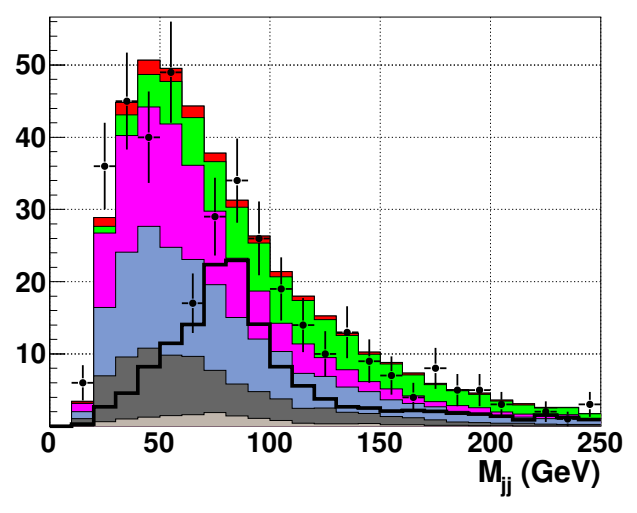

(a)

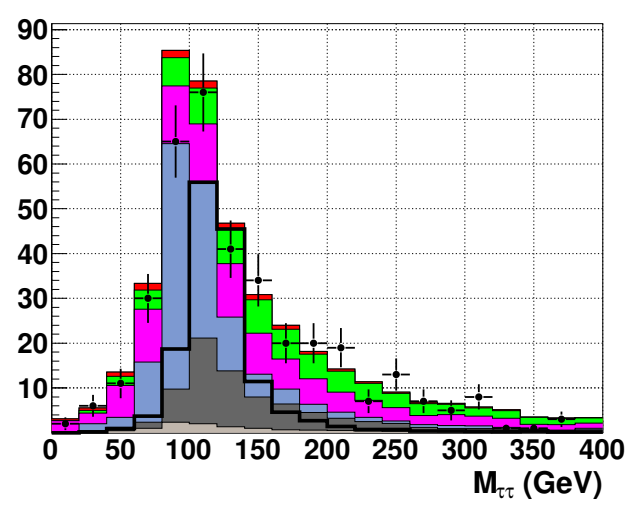

(c)

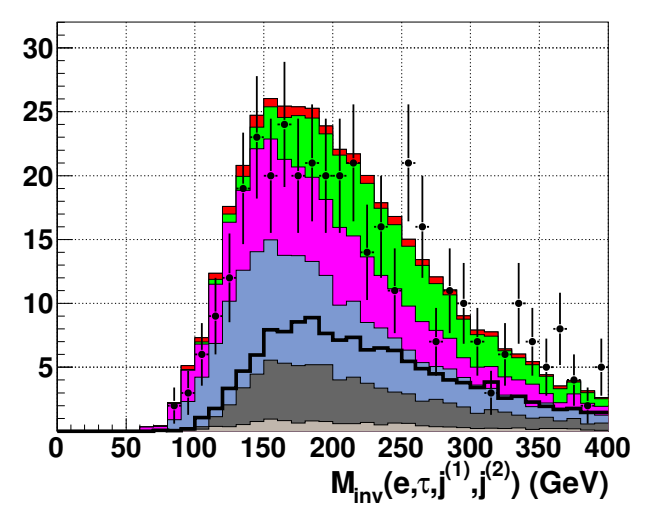

(b)

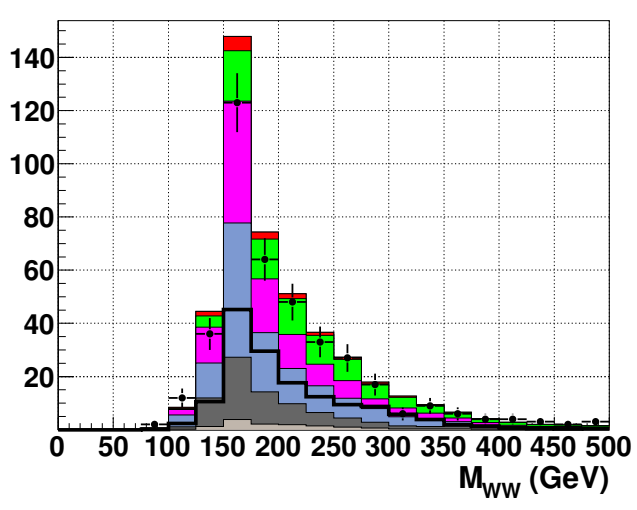

(d)

Figure 5.7. Invariant mass distributions of the two jets, electron + tau + jets, $\tau \tau$, and $W W$. The signals are shown multiplied by 250 . 


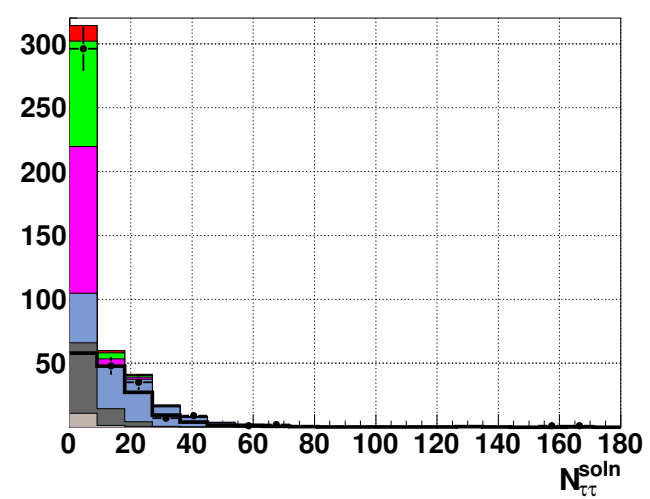

(a)

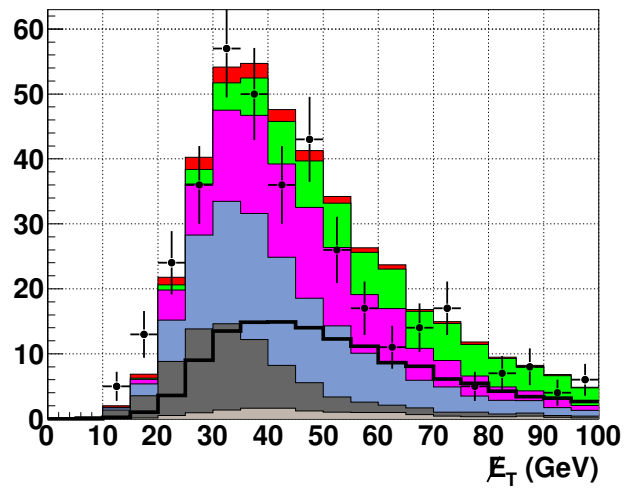

(c)

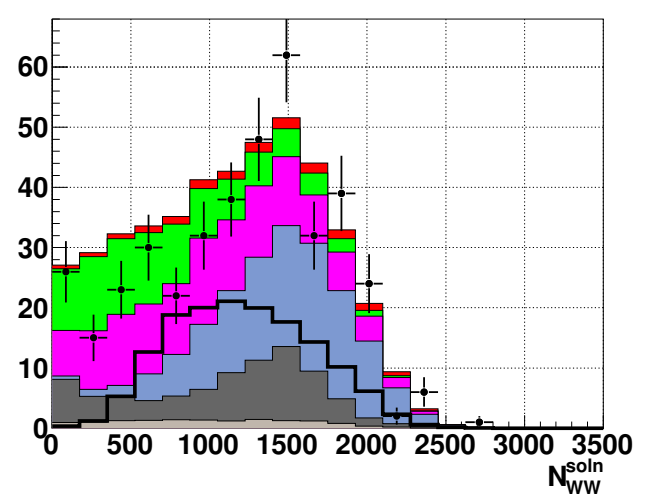

(b)

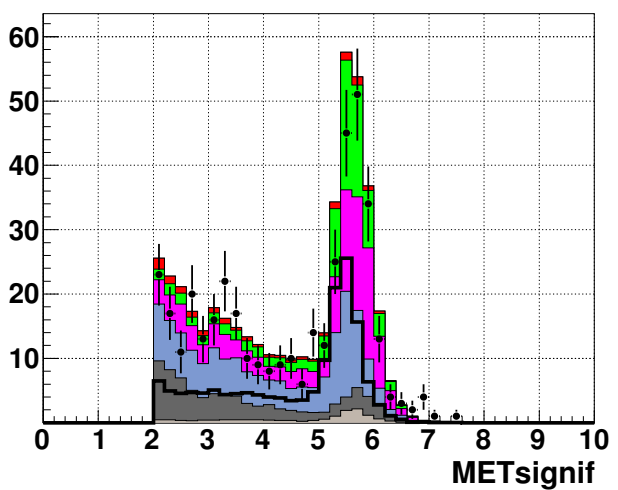

(d)

Figure 5.8. The number of physical solutions, $\mathbb{E}_{T}$ and $\mathcal{S}$ distributions. The signals are shown multiplied by 250 . 


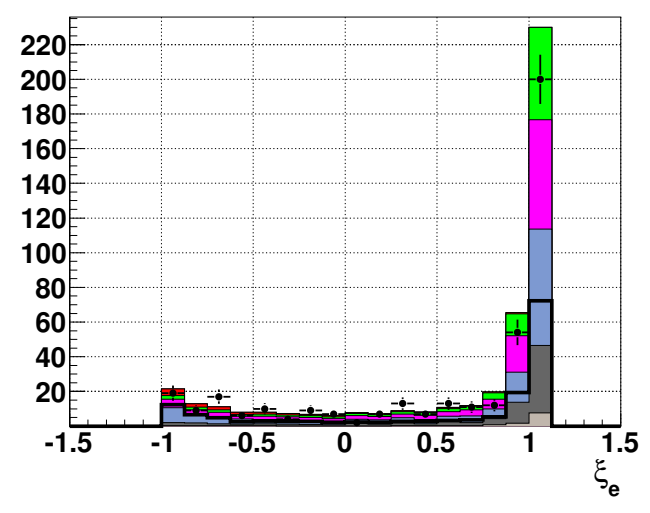

(a)

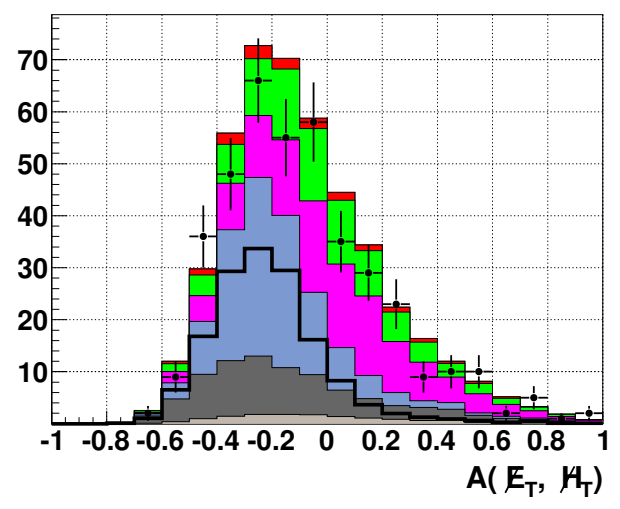

(c)

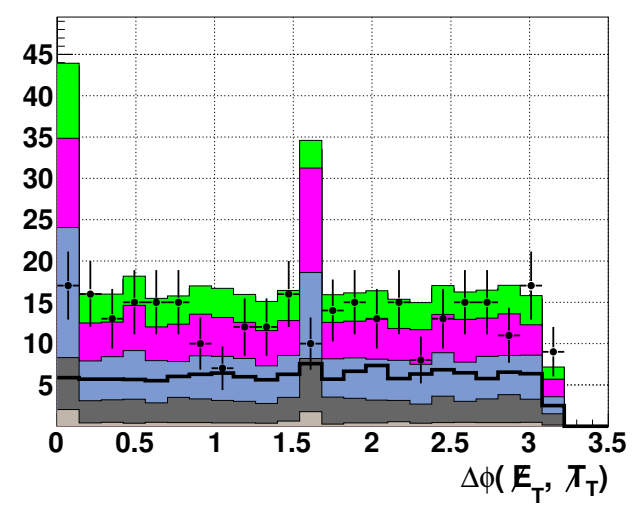

(b)

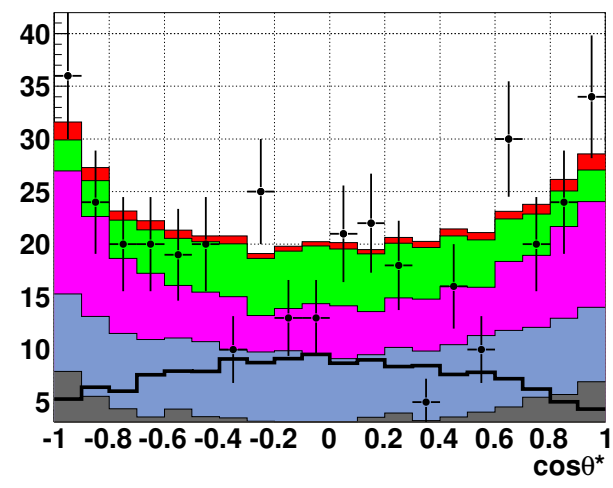

(d)

Figure 5.9. Electron MVA, missing energy $\Delta \phi$, missing energy asymmetry, and $\cos \theta^{*}$. The signals are shown multiplied by 250 . 


\subsection{MVA Strategy}

The general procedure used in MVA Higgs searches is to develop a new output or "final discriminant" at each Higgs mass in $5 \mathrm{GeV}$ intervals from 105-150 GeV. Each $m_{H}$ point uses the same events but depending on the multivariate training could have very different outputs. The final discriminants are then used to calculate the limits (see Chapter 6) as a function of $m_{H}$.

The training procedure has a great effect on the final limits and therefore many techniques were attempted. An outline of the final MVA strategy is shown in Fig. 5.10. The final procedure as well as other attempted MVA techniques are discussed in the following sections.

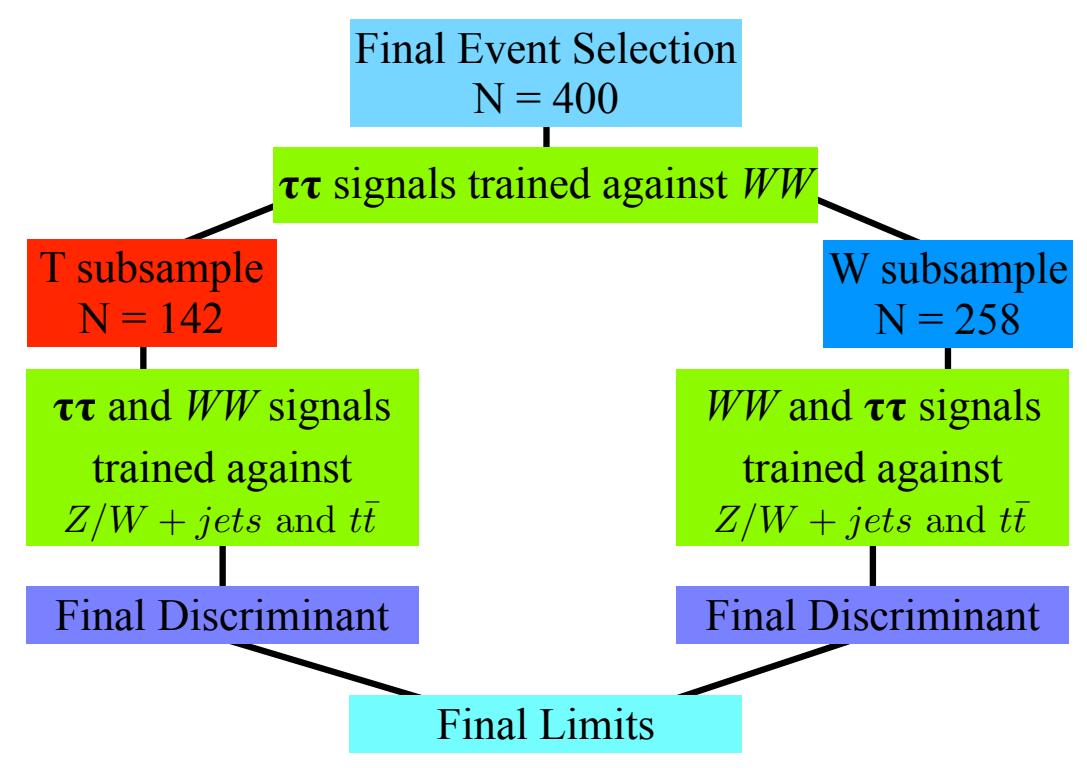

Figure 5.10. Schematic of the final MVA procedure. The final sample will be split, each sample will develop its own limits, and the two subsamples will be recombined to set the final limits. 


\subsection{T and W Subsamples}

To optimize the sensitivity of this search the data are separated into two orthogonal samples using a $\mathrm{BDT}$ that trains $H \rightarrow \tau \tau$ signals against $H \rightarrow W W$ signals. The sum of Higgs signals at all mass points is used. The resulting BDT is shown in Fig. 5.11, where $\tau \tau$ signals are pushed towards +1 , and $W W$ signals are pushed towards -1 . Despite not having a symmetric separation this BDT output is still capable of creating highly enriched $\tau \tau$ and $W W$ subsamples. In this distribution the $Z \rightarrow \tau \tau$ background peaks on the positive side showing a tendency of the BDT training to handle $Z \rightarrow \tau \tau$ events in a similar way as $H \rightarrow \tau \tau$, as expected.

Events with a value of $\mathrm{BDT}_{\mathrm{TW}}>-0.6$ make up the $\tau \tau$ dominated subsample (called the $\mathrm{T}$ subsample) and the events with $\mathrm{BDT}_{\mathrm{TW}}<-0.6$ constitute the $W W$ dominated subsample (called the $\mathrm{W}$ subsample). This value was chosen to make the statistics in each sample comparable and so that the purity of each subsample was high. Table 5.1 summarizes different choices of this value and its limited effect on the background yields and the purity. Purity is defined as the amount of one signal in the subsample to the total in that subsample. Both samples have a purity above $90 \%$ in their dominant signal mass regions respectively. The T subsample has 142 events with over $50 \%$ of the background begin $Z \rightarrow \tau \tau$, and the $\mathrm{W}$ subsample has 258 events with roughly $40 \%$ of the background being $W+$ jets.

This separation technique was shown to have about a 10-20\% improvement over the non-separated case. Figure 5.12 compares the expected and observed limits with and without using the $\mathrm{BDT}_{\mathrm{TW}}$ method. Since the region above $m_{H}=150 \mathrm{GeV}$ was largely excluded by both Tevatron and LHC experiments the focus turned to $m_{H}<150 \mathrm{GeV}$. The $\mathrm{BDT}_{\mathrm{TW}}$ method has better overall sensitivity and does not increase with $m_{H}$ as drastically as the non-separated technique. 
It is important to note that this BDT does not serve to set limits, but only creates the subsamples. The two subsamples are individually trained again to create a final discriminant used for limit setting. Finally, the limits from each subsample are combined to form the final limit (see Chapter 6).

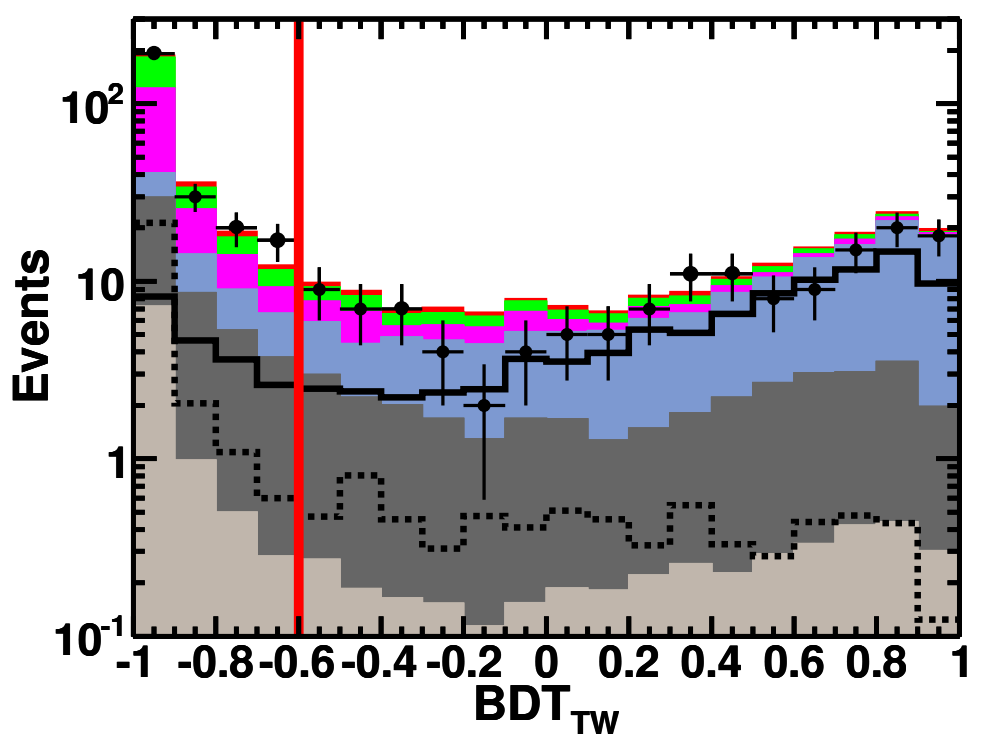

Figure 5.11. BDT trained to discriminate $\tau \tau$ signals from $W W$ signals using all signal events between 105 and $150 \mathrm{GeV}$. For this plot only, the sum of $\tau \tau$ signals is shown in the solid line and the sum of all $W W$ signals is shown in the dotted line. The red line shows the separation of the two subsamples.

\subsection{Initial BDT Studies}

The largest contributing factor to any improvements or degradations to the limit in this analysis come from the BDT training procedure. A different technique was used in every previous version of this analysis. During the latest implementation the principle technique attempted was to use a BDT training at each $m_{H}$ where the sum of all signals $\mathrm{WH}_{\tau \tau}, \mathrm{ZH}_{\tau \tau}, \mathrm{VBF}_{\tau \tau}, \mathrm{GGF}_{\tau \tau}, \mathrm{WH}_{W W}, \mathrm{ZH}_{W W}, \mathrm{VBF}_{W W}$ and 
Table 5.1. Effect of different selections $(-0.8,-0.6,-0.5)$ of $\mathrm{BDT}_{\mathrm{TW}}$ on background yields and purity.

\begin{tabular}{|c||c|c||c|c||c|c|}
\hline \hline \multicolumn{1}{|c||}{ Sample } & \multicolumn{2}{c||}{-0.8} & \multicolumn{2}{c||}{-0.6} & \multicolumn{2}{c|}{-0.5} \\
\hline & $\tau \tau$ & $W W$ & $\tau \tau$ & $W W$ & $\tau \tau$ & $W W$ \\
\hline \hline$t \bar{t}$ & 21 & 69 & 15 & 75 & 13 & 76 \\
$W$ jets & 24 & 93 & 16 & 100 & 14 & 102 \\
$Z_{\text {eejets }}$ & 39 & 30 & 31 & 38 & 28 & 41 \\
$Z_{\tau \tau}$ jets & 113 & 17 & 107 & 23 & 104 & 26 \\
DB & 5 & 8 & 4 & 9 & 4 & 9 \\
MJ & 20 & 0 & 19 & 0 & 19 & 0 \\
$\Sigma$ bkgd & 222 & 215 & 191 & 246 & 182 & 254 \\
Data & 179 & 221 & 142 & 258 & 133 & 267 \\
\hline $\mathrm{P}_{125}$ & $92.2 \%$ & $64.7 \%$ & $93.2 \%$ & $57.0 \%$ & $93.5 \%$ & $54.2 \%$ \\
$\mathrm{P}_{145}$ & $63.3 \%$ & $93.9 \%$ & $68.8 \%$ & $91.3 \%$ & $70.4 \%$ & $90.4 \%$ \\
\hline \hline
\end{tabular}

$\mathrm{GGF}_{W W}$ was trained against the sum of the major backgrounds $Z+$ jets, $W+$ jets, $t \bar{t}$ and MJ. This procedure is computationally straight forward and produces final limits with better sensitivity compared to those of any MVA.

One significant drawback is shown in Fig. 5.13 (a) and (b) which show a strong oscillatory behavior in both the cross section limit and log-likelihood ratio (LLR) plots (see Chapter 6 for limit/LLR description). The oscillations were concluded to have originated from the BDT training procedure, after a cross check of the final limits using the di-jet mass distribution showed monotonically changing limits with increasing mass. Table 5.2 shows the final limits using the di-jet mass as the final discriminant for both the $\mathrm{T}$ and $\mathrm{W}$ subsamples. In general the limits are about a factor two worse than any BDT method, and they increase or decrease proportional to the amount of signal expected in the subsample. ${ }^{1}$

Figure 5.13 (c) and (d) show one attempted version at smoothing the mass dependence of the limit and LLR distributions. Rather than using signal distributions

\footnotetext{
${ }^{1}$ The degradation illustrates why a multivariate approach is used in this analysis.
} 

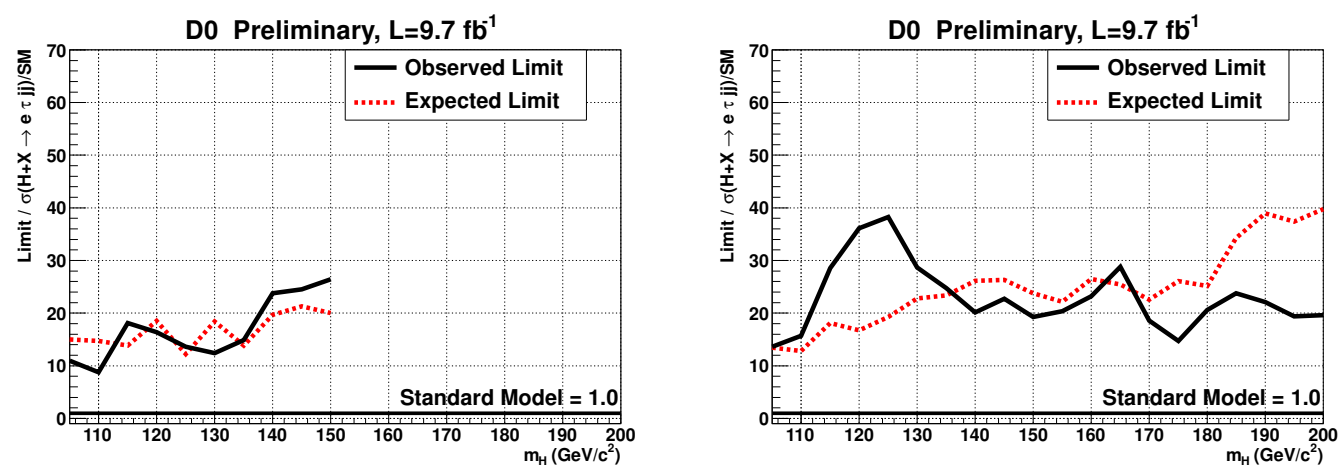

Figure 5.12. Comparison of limits using the $\mathrm{BDT}_{\mathrm{TW}}$ method. (a) Combination of the subsamples and (b) no separation. The expected limit improves by $\sim 10 \%$ when using the $\mathrm{BDT}_{\mathrm{TW}}$.

from a single mass point a weighted average (50\% from $m_{H}$, and $25 \%$ each from $\left.m_{H} \pm 5\right)$ of the signals is used. The weighted average of these signals is then trained against the dominant backgrounds as before. Clearly this "averaging" technique only marginally helped reduce the oscillatory behaviors. In order to alleviate this problem an alternative approach was sought.

\subsection{Global BDT Method}

Nearly all Higgs searches at the Tevatron train individual MVAs for Higgs masses in $5 \mathrm{GeV}$ intervals from $100-150$ or $200 \mathrm{GeV}$ [31], [32]. For searches with one single production mode, and one decay mode this technique may be sufficient, but for those that involve multiple production and decay modes significant issues arise. The dominant production process will certainly change with $m_{H}$, causing differences across a broad range. Also individual mass point trainings can have small variations that drastically effect the final result. For this analysis in particular, with three production channels and two decay modes the discontinuities that arise from separate BDT training at each mass point produce unphysical oscillations of the limits and 


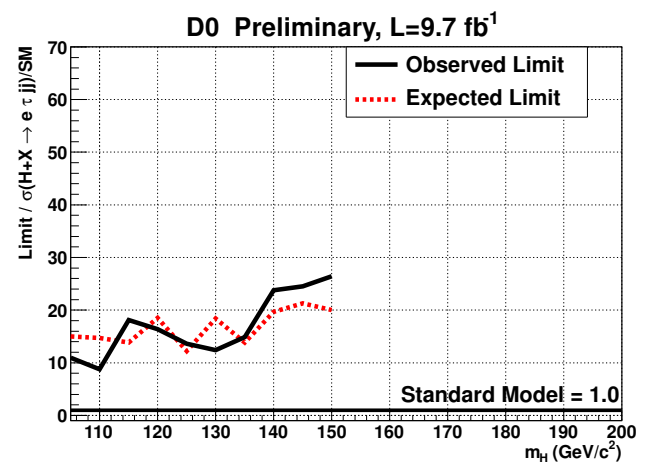

(a)

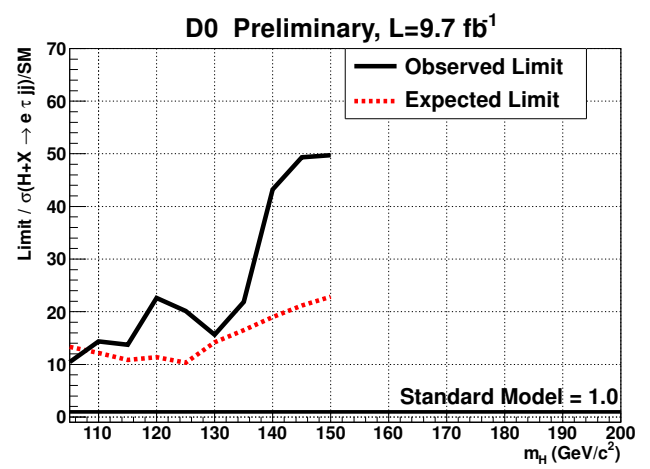

(c)

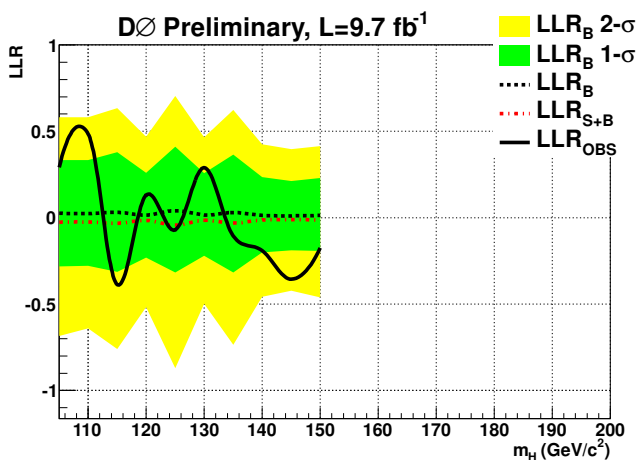

(b)

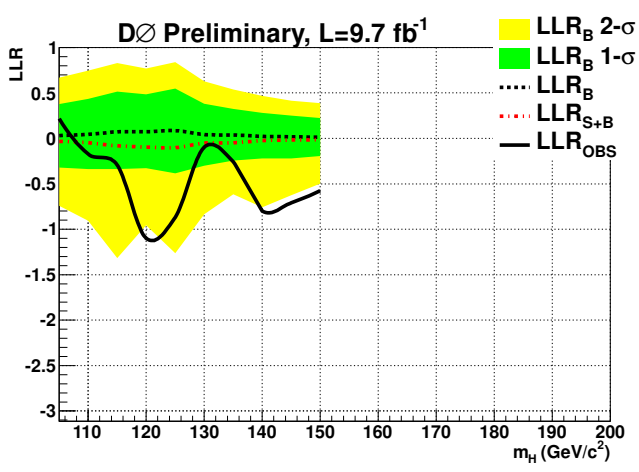

(d)

Figure 5.13. The limit and LLR distributions for two early BDT techniques, (a), (b) training at each mass point; (c), (d) training at each mass point with $50 \%$ contribution from the point and $25 \%$ from $m_{H} \pm 5 \mathrm{GeV}$. The averaging technique only marginally helped reduce the oscillatory behaviors. Compare to Fig. 6.3.

the LLR. Namely, fluctuations of data points in high BDT value bins can cause dramatic changes in the observed limits, showing a data excess where one may not exist (see Section 6.2). A method was devised to alleviate these fluctuations that should generally be useful in any search that uses MVA trainings as a functions or some parameter, especially new physics searches at the LHC.

A BDT is trained with the 32 variables listed in Section 5.1. For each subsample the sum of the eight major signals processes at all mass points is trained against the sum of the dominant backgrounds: for example $\mathrm{GGF}_{\tau \tau}, \mathrm{VBF}_{\tau \tau}, \mathrm{ZH}_{\tau \tau}, \mathrm{WH}_{\tau \tau}$, $\mathrm{GGF}_{W W}, \mathrm{VBF}_{W W}, \mathrm{ZH}_{W W}$ and $\mathrm{WH}_{W W}$ against $Z+$ jets, $W+$ jets, and $t \bar{t}$. In the T 
Table 5.2. Expected limits for the $\mathrm{T}$ and $\mathrm{W}$ subsamples using the di-jet mass as the final discriminant. There are no signs of oscillation.

\begin{tabular}{c|cc}
\hline \hline \multicolumn{3}{c}{ dijet mass limits } \\
\hline mass (GeV) & exp. (T) & exp (W) \\
\hline 105 & 19.068 & 111.657 \\
110 & 19.665 & 111.467 \\
115 & 20.601 & 103.971 \\
120 & 24.064 & 82.421 \\
125 & 26.817 & 70.620 \\
130 & 30.670 & 59.300 \\
135 & 35.285 & 52.540 \\
140 & 45.301 & 43.733 \\
145 & 56.530 & 40.991 \\
150 & 76.127 & 32.945 \\
\hline \hline
\end{tabular}

subsample there is some contamination from $W W$ signals $(<10 \%)$ and vice-a-versa for the $\mathrm{W}$ subsample. Those processes contributing very little to either the signal or background are not included, i.e. HZ, MJ and DB.

The unique feature of the so-called "Global BDT" technique is that it uses a single BDT training for all values of $m_{H}$. It incorporates $m_{H}$ as an input variable to the BDT training, then evaluates the BDT at each $m_{H}$. Since the sum of signals from each mass point is used to train the BDT there is a distribution of $m_{H}$ for each subsample. Figure 5.14 shows the background (red) and signal (blue) distributions of $m_{H}$ used for training. The $m_{H}$ distribution for background was normalized to match the expected $m_{H}$ signal distribution (see Tab. 4.5 and 4.6). This is necessary so that no artificial discrimination is introduced to the BDT because of $m_{H}$. The small discrepancies are consistent within the statistical fluctuations.

The Global BDT method successfully removes the variations in BDT trainings across mass points by using a single BDT training that is evaluated at each $m_{H}$ rather than a separate training at each $m_{H}$. It provides a more uniform limit on the Higgs 
cross section and it achieves this goal with minimal $(<10 \%)$ degradation of the limits compared to the standard mass point trainings.

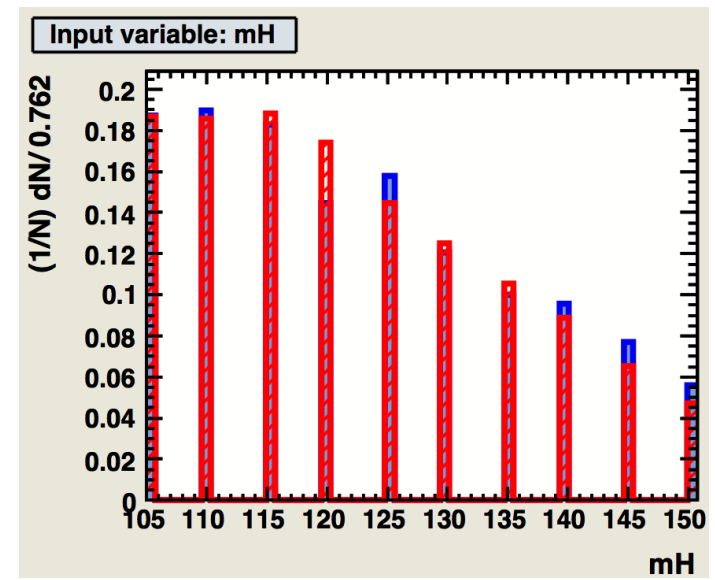

(a)

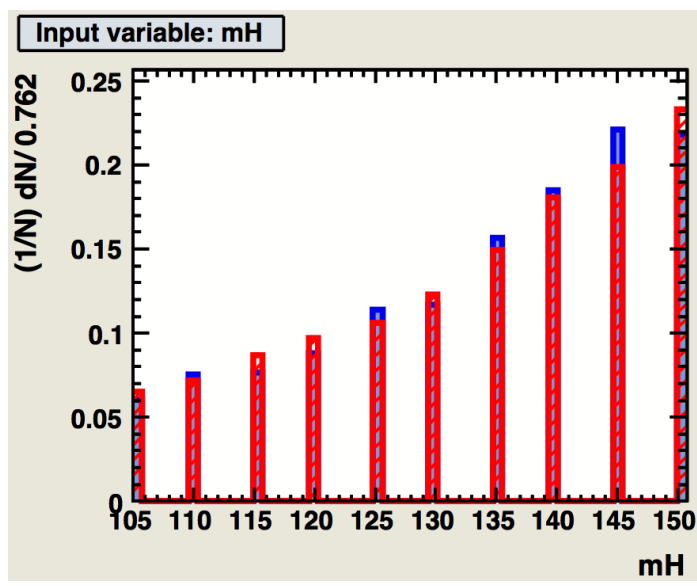

(b)

Figure 5.14. $m_{H}$ distributions used for BDT training for (a) T subsample and (b) W subsample. The background (red) was normalized to the signal (blue) to not allow the BDT artificially good separation between the two.

\subsection{BDT Outputs}

Figures 5.15 - 5.24 show the final BDT output for the $\mathrm{T}$ and $\mathrm{W}$ subsamples at each Higgs mass from 105 - $150 \mathrm{GeV}$. The T subsample uses uniform bin sizes of 0.05, while the $\mathrm{W}$ subsample uses 0.05 bins from -1 to 0.4 , then has bins of 0.1 and 0.2 . The binning was developed for non-global BDT methods which could become biased in the case of small MC statistics, although the Global BDT method reduces this bias, the same binning is kept for consistency. The variable BDT output has only a minimal effect on the cross section limits. 


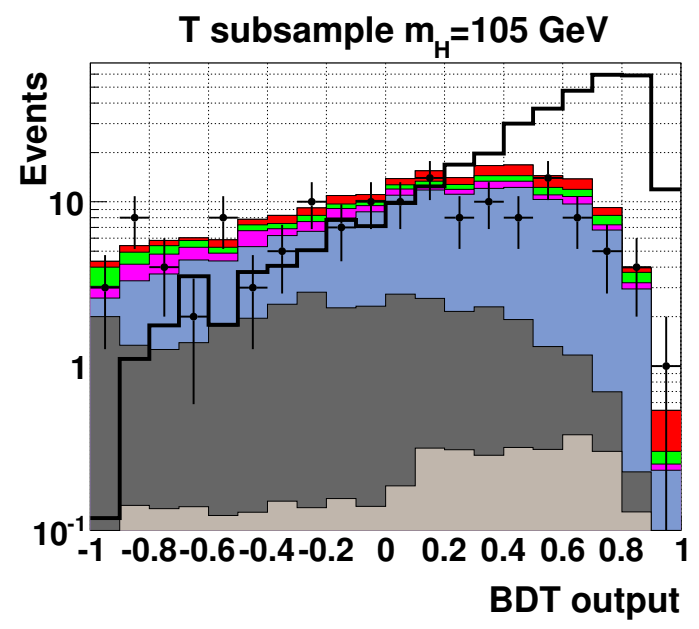

(a)

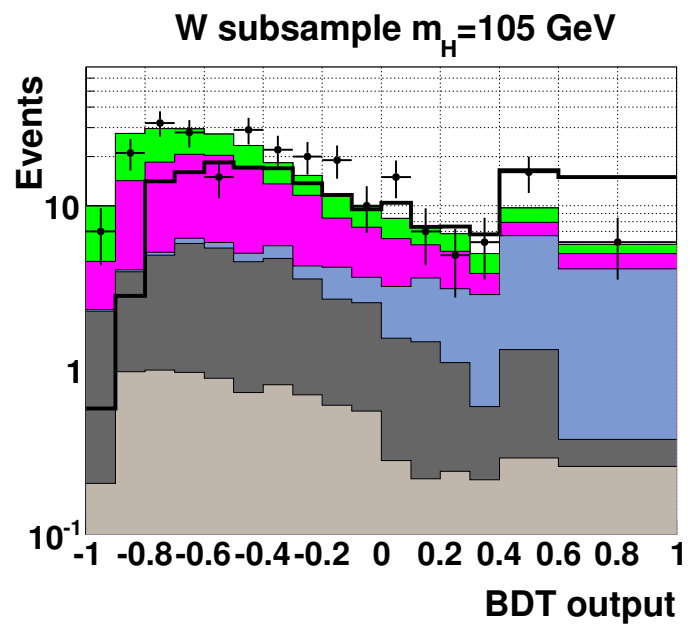

(b)

Figure 5.15. BDT outputs for $m_{H}=105 \mathrm{GeV}$. (a) The T subsample output, (b) the W subsample output. The signals are shown multiplied by 250 .

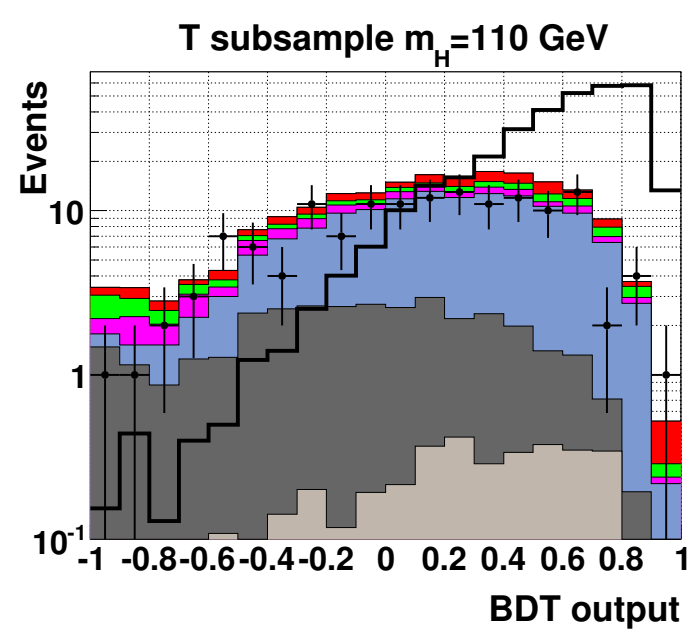

(a)

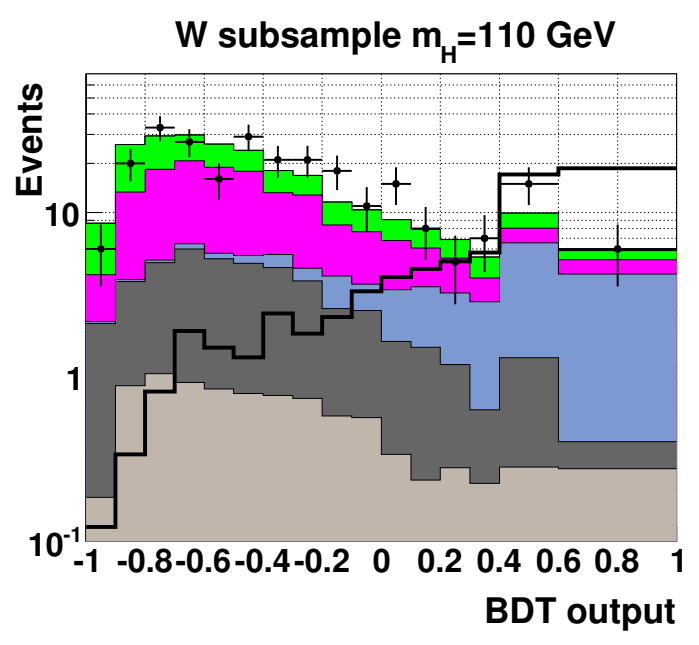

(b)

Figure 5.16. BDT outputs for $m_{H}=110 \mathrm{GeV}$. (a) The T subsample output, (b) the W subsample output. The signals are shown multiplied by 250 . 


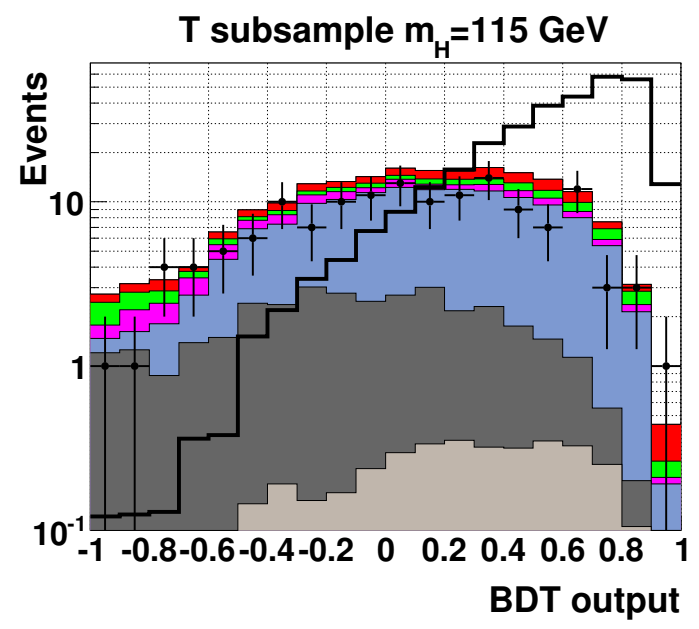

(a)

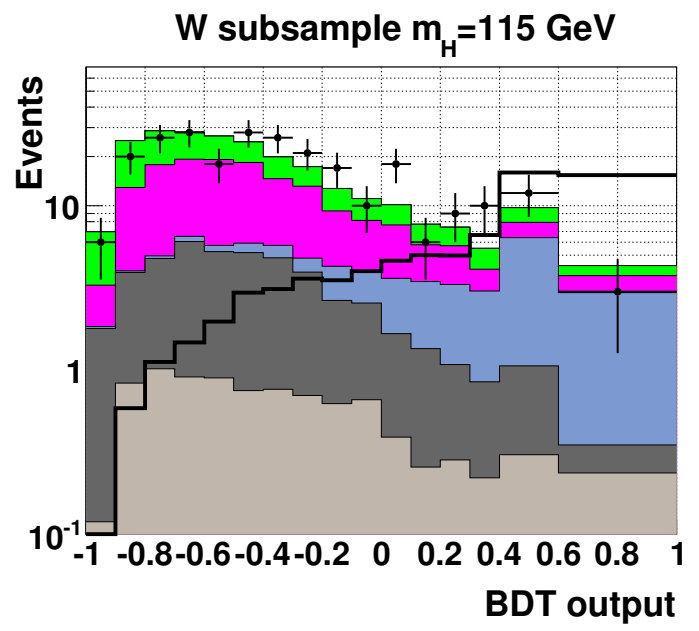

(b)

Figure 5.17. BDT outputs for $m_{H}=115 \mathrm{GeV}$. (a) The T subsample output, (b) the W subsample output. The signals are shown multiplied by 250 .

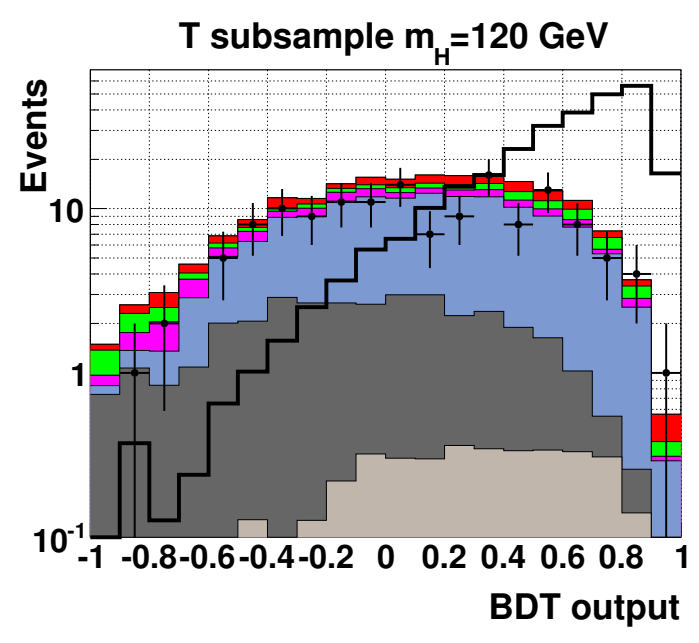

(a)

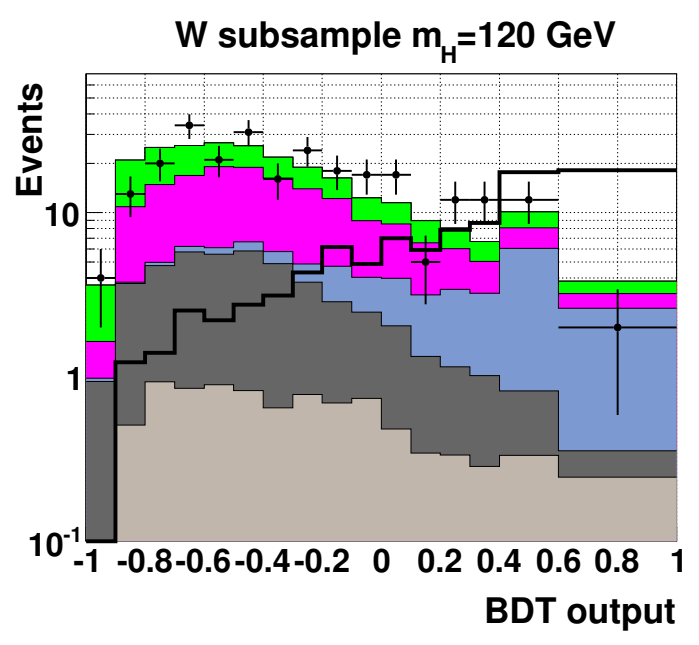

(b)

Figure 5.18. BDT outputs for $m_{H}=120 \mathrm{GeV}$. (a) The T subsample output, (b) the W subsample output. The signals are shown multiplied by 250 . 


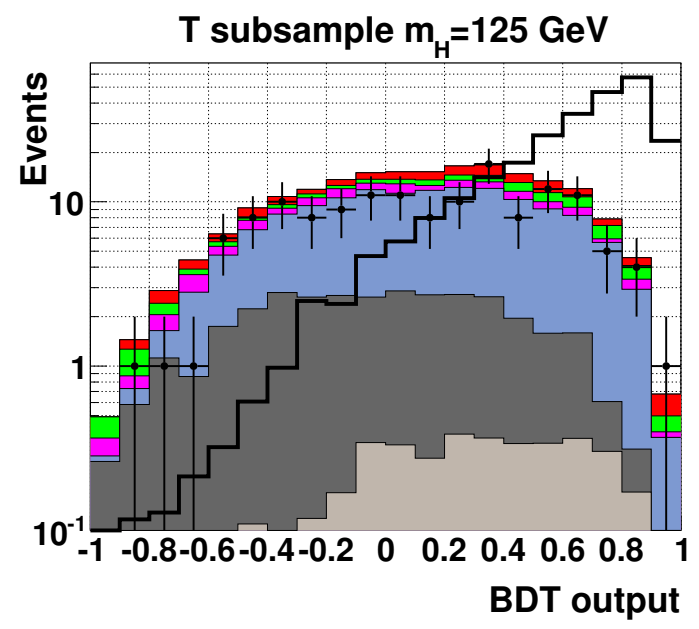

(a)

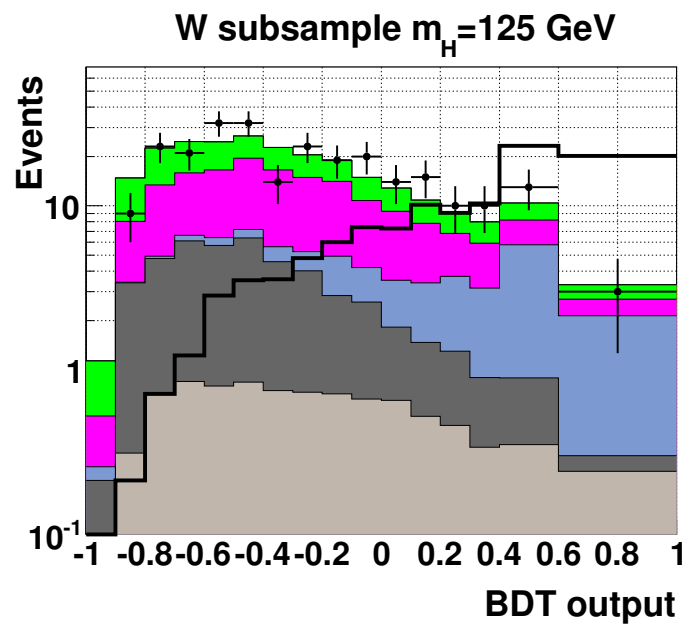

(b)

Figure 5.19. BDT outputs for $m_{H}=125 \mathrm{GeV}$. (a) The T subsample output, (b) the W subsample output. The signals are shown multiplied by 250 .

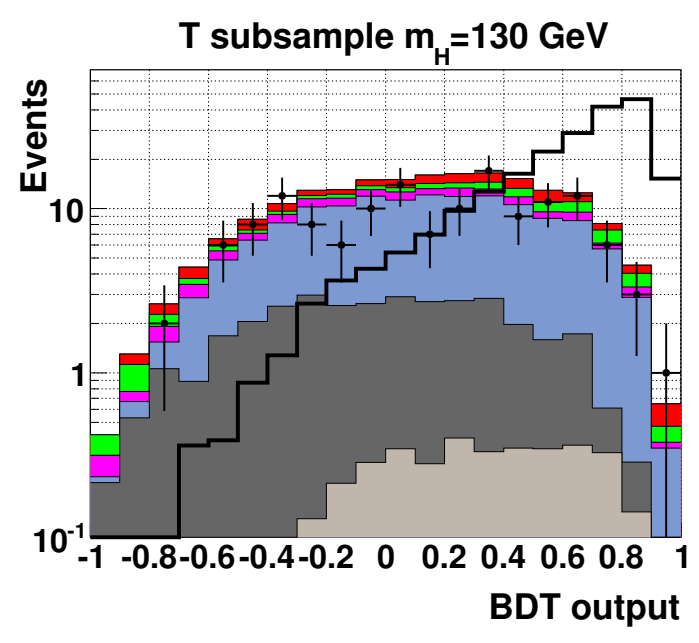

(a)

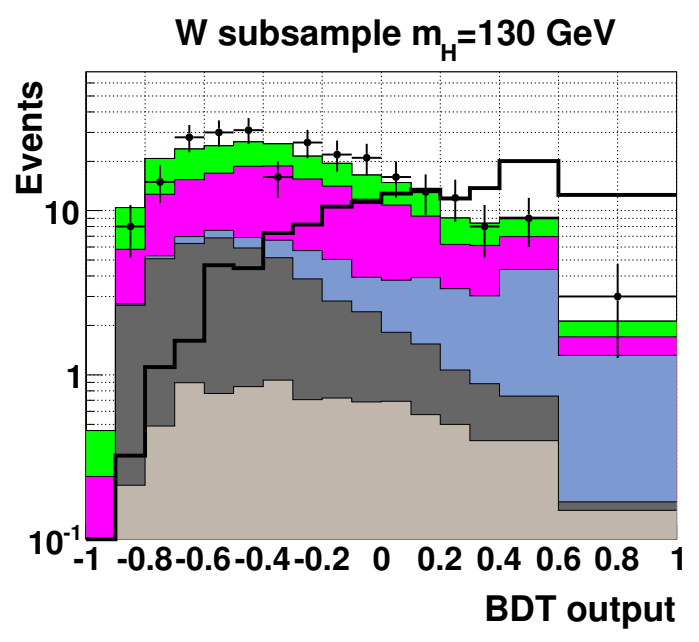

(b)

Figure 5.20. BDT outputs for $m_{H}=130 \mathrm{GeV}$. (a) The T subsample output, (b) the W subsample output. The signals are shown multiplied by 250 . 


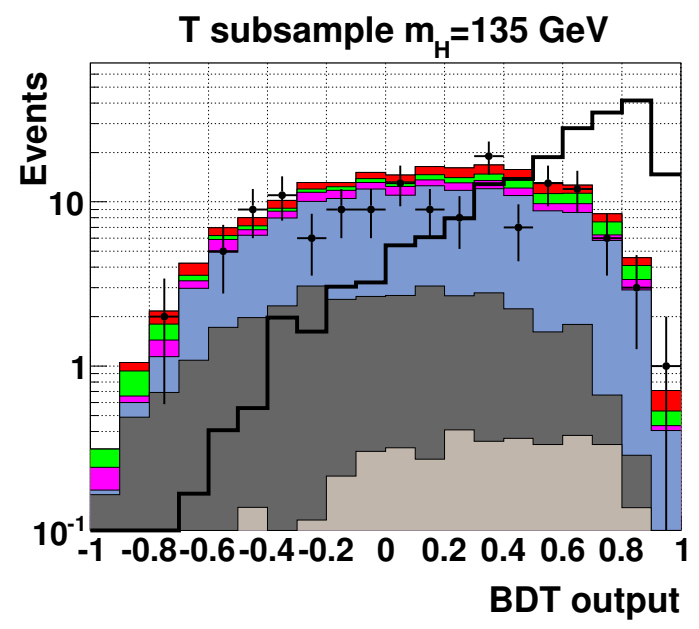

(a)

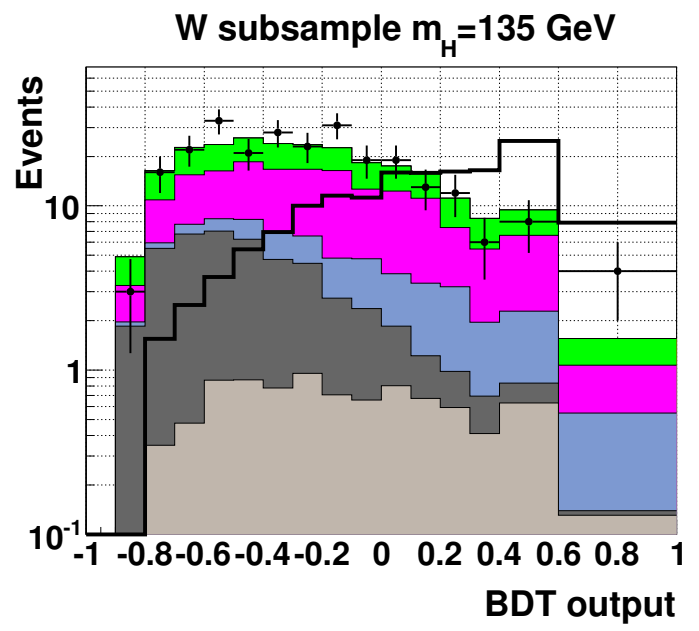

(b)

Figure 5.21. BDT outputs for $m_{H}=135 \mathrm{GeV}$. (a) The T subsample output, (b) the W subsample output. The signals are shown multiplied by 250 .

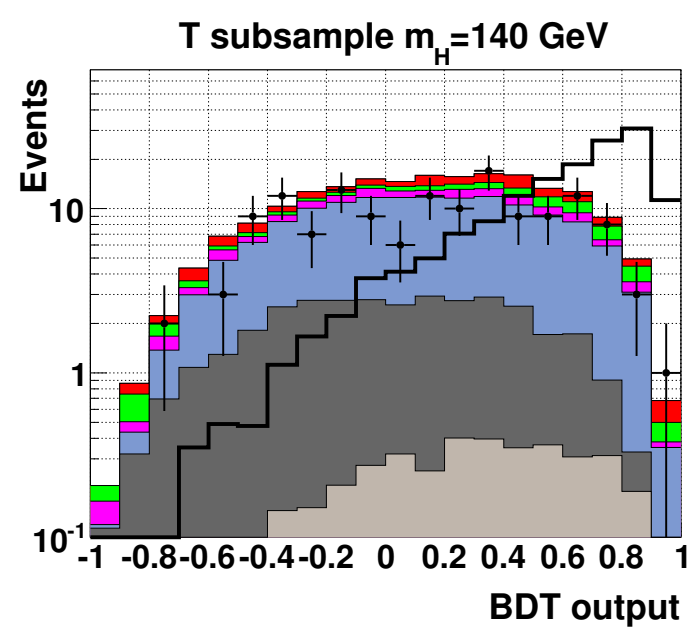

(a)

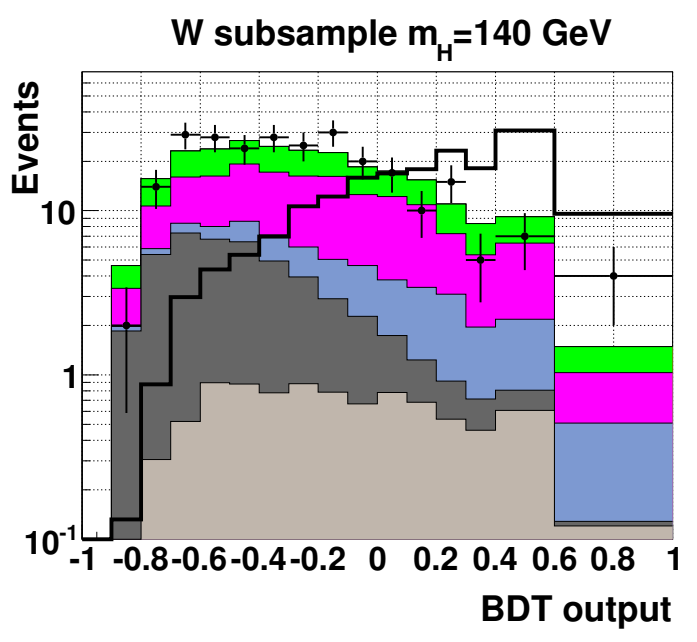

(b)

Figure 5.22. BDT outputs for $m_{H}=140 \mathrm{GeV}$. (a) The T subsample output, (b) the W subsample output. The signals are shown multiplied by 250 . 


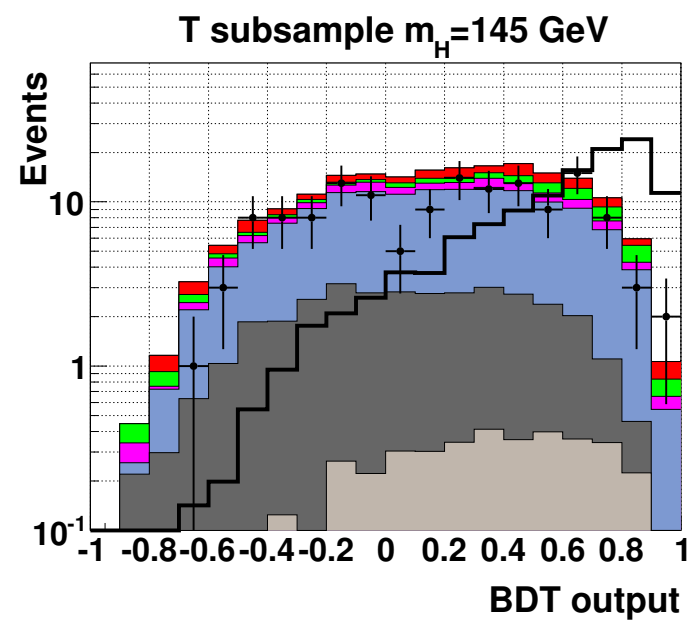

(a)

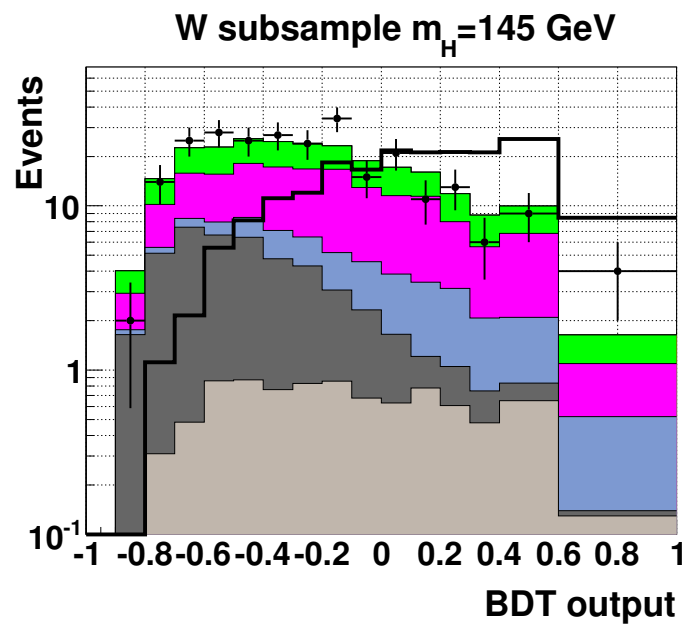

(b)

Figure 5.23. BDT outputs for $m_{H}=145 \mathrm{GeV}$. (a) The T subsample output, (b) the W subsample output. The signals are shown multiplied by 250 .

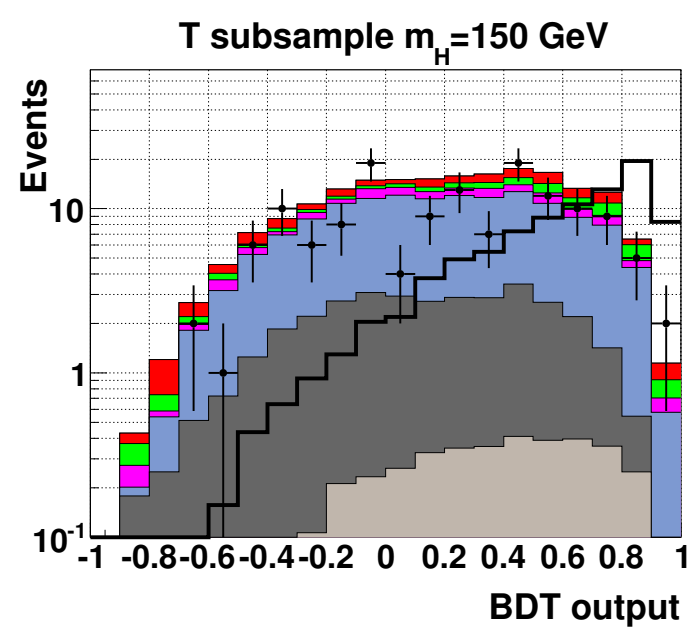

(a)

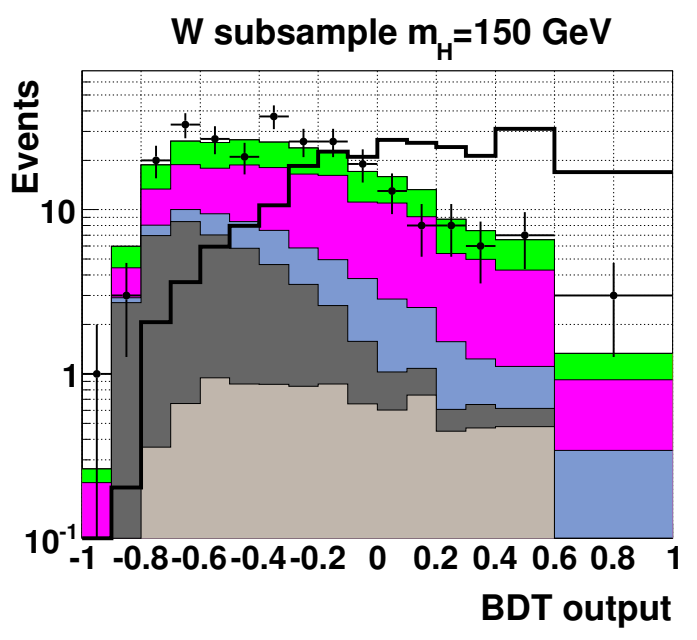

(b)

Figure 5.24. BDT outputs for $m_{H}=150 \mathrm{GeV}$. (a) The T subsample output, (b) the W subsample output. The signals are shown multiplied by 250 . 
The shape of the $\mathrm{T}$ and W BDT distributions varies slowly and continually as $m_{H}$ increases, thus achieving the desired result. To contrast the Global BDT with the previous technique of a new training at each mass point, the BDTs for the T (W) subsample at $m_{H}=115 \mathrm{GeV}$ and $m_{H}=120 \mathrm{GeV}$ (140 and 145) are shown in Fig. 5.25. The adjacent mass point trainings using the Global BDT (Fig. 5.25 (a),(b) and $(\mathrm{e}),(\mathrm{f}))$ technique are remarkably similar. The shapes of both the signal and the background change by only a small amount. In the case of the nominal BDT method (Fig. $5.25(\mathrm{c}),(\mathrm{d})$ and $(\mathrm{g}),(\mathrm{h}))$ it is clear how the shape of both the background and the signal change.

Without the Global BDT, there is a potential for large fluctuations of BDT output values between neighboring mass points. To highlight this point consider the data events listed in Tab. 5.3. These are the two highest valued data events at $m_{H}=115$ and $120 \mathrm{GeV}$ for the Global BDT. While event 193157 has similar values for both mass points the same is not true of event 209069. In this event the nominal BDT method shows a drastic change in adjacent mass point BDT output value, whereas the Global BDT shows a stable output. This serves as an example of how events can be classified in drastically different ways when using different BDT trainings at adjacent mass points. Removing the instability of individual events greatly reduces the limit/LLR oscillations, and prevents a false excess in observed limits which previously could occur if even one event fluctuated to a high BDT value.

Table 5.3. Highest BDT value event comparison of Global BDT versus nominal. The Global BDT values remain essentially the same, while the nominal method can have severe fluctuations.

\begin{tabular}{c|cc|cc}
\hline \hline Event & GBDT $_{115}$ & GBDT $_{120}$ & BDT $_{115}$ & BDT $_{120}$ \\
\hline 193157 & 0.916 & 0.915 & 0.709 & 0.706 \\
209069 & 0.814 & 0.817 & 0.814 & 0.467 \\
\hline \hline
\end{tabular}




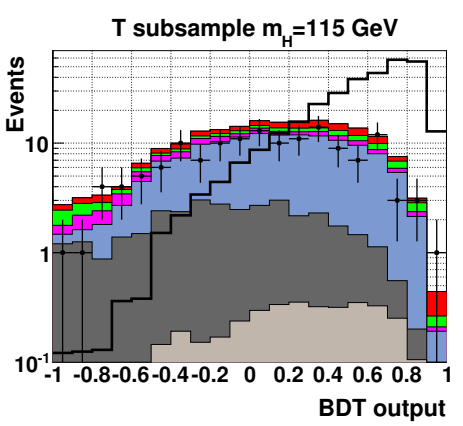

(a)

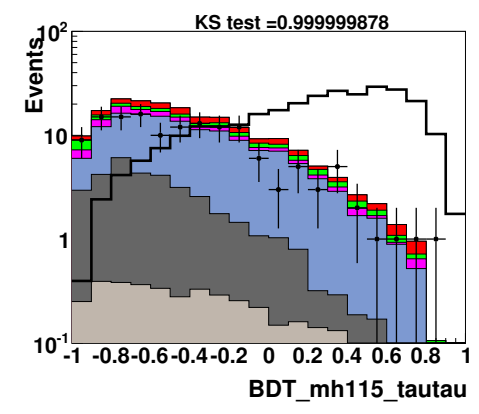

(c)

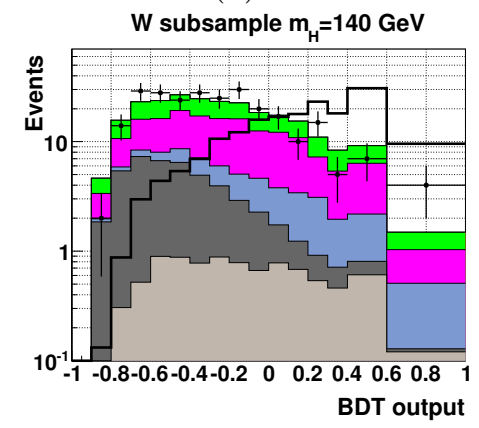

(e)

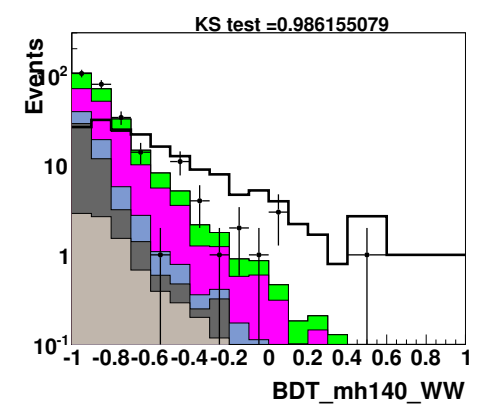

$(\mathrm{g})$

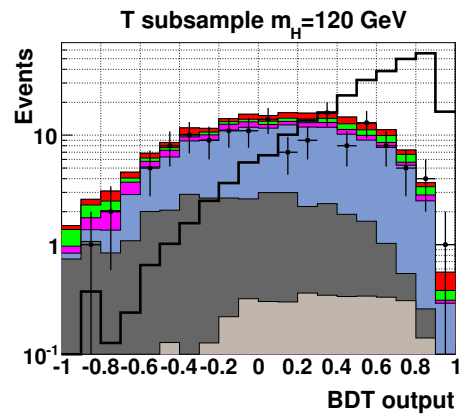

(b)

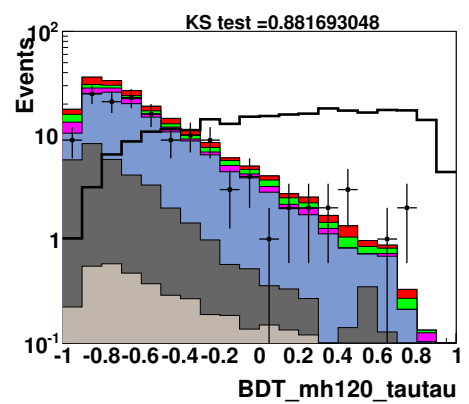

(d)

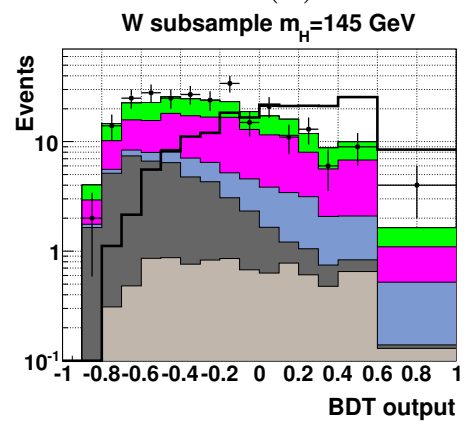

(f)

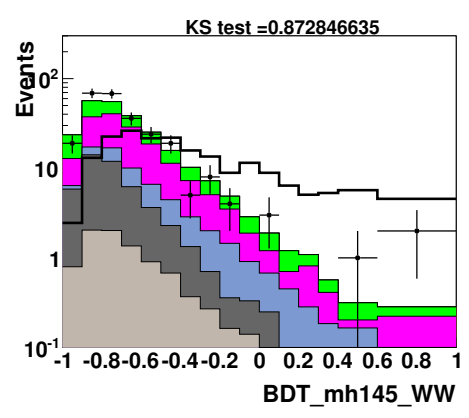

(h)

Figure 5.25. Comparison of adjacent mass point BDT outputs: [(a), (b), (e) and (f)] with the Global BDT and $[(\mathrm{c}),(\mathrm{d}),(\mathrm{g})$ and $(\mathrm{h})]$ with individual mass point trainings. The shape change of the signal and background without the Global BDT from $m_{H}=115$ to $120 \mathrm{GeV}$ (140 to 145$)$ in the $\mathrm{T}(\mathrm{W})$ subsample is one example of the large variability in adjacent mass point training. 


\section{CHAPTER 6}

\section{LIMIT CALCULATIONS}

\subsection{Systematic Uncertainties}

The total systematic uncertainty in this analysis is comprised of many different terms that fall into two broad categories: flat (normalization) systematics are constants that have the same value independent of BDT output value, while shape systematics vary across BDT output value and are computed by shifting each systematic by $\pm 1 \sigma$ and observing how the Global BDT output changes. A list of all the sources considered in this analysis and their type is included in Tab. 6.1. While it is important to carefully understand all the sources of systematic uncertainty, it is worth noting that the MVA techniques have a far greater impact on the overall sensitivity of this search.

\subsubsection{Normalization Systematics}

Luminosity - There is a $6.1 \%$ uncertainty in the total integrated luminosity, composed of $4.1 \%$ from the $\mathrm{D} \varnothing$ determination and $4.6 \%$ uncertainty in the luminosity cross section which is fully correlated with CDF.

Electron ID/Tracking/Iso - The electron identification, track matching, and isolation are determined from a sample of $Z \rightarrow e e$ enriched events. Using this sample, the EM ID group obtained a $4 \%$ uncertainty in this quantity.

Electron Trigger - The EM ID group also determined a $2 \%$ uncertainty of the EJetsOR trigger.

Tau ID - From a sample of $Z \rightarrow \tau \tau$ enriched events the Tau ID group determined the 
total tau identification uncertainty coming from three sources: (1) MC selection efficiency, (2) MJ background determination and (3) the $p_{T}^{\tau}$ dependence. The systematic coming from the MC selection is defined as

$$
\frac{1-\text { effNN }_{\mathrm{x}}}{3}
$$

where effNN $\mathrm{x}_{\mathrm{x}}$ is the efficiency for the selection on $\mathrm{NN}_{\tau}$ at a value of $\mathrm{x}$, and the factor 3 is a "best guess" based on averaging. Since this selection is made just once, it does not vary as a function of $\mathrm{NN}_{\tau}$. Two methods are used to calculate the MJ background contribution. The difference between these estimates is taken as the systematic uncertainty. Finally the $\mathrm{MC}$ correction factors are rederived for two $p_{T}$ regions: $p_{T}^{\tau}<25$ $\mathrm{GeV}$ and $p_{T}^{\tau}>25 \mathrm{GeV}$ and the minimal difference to the nominal correction is taken as the systematic. The MJ and $p_{T}$ systematic vary as a function of $\mathrm{NN}_{\tau}$, and are added in quadrature with the MC selection systematic. The total tau ID systematic uncertainty by type ${ }^{1}$ is then $5.5 \%, 4.0 \%$ and $6.0 \%$, or an average of $4.6 \%$. $^{2}$

Tau Tracking and Energy Scale - The tau tracking uncertainty is $1.4 \%$. The average uncertainty, weighted by tau type, for the tau energy scale variation is $9.8 \%$. Cross Sections - The signal and background cross section uncertainties are taken from the standard Tevatron Physics Working Group (TeVN-PHWG) and Higgs group as shown in Tab. 6.1.

Parton Distribution Functions - The uncertainty due to the PDFs is obtained from the CTEQ pdf eigenvector set. Signal and background shape differences from the nominal BDT are computed separately and are $1.6 \%$ and $2.0 \%$, respectively.

MJ Normalization - There are three components to the MJ uncertainty: (1) the uncertainty on $\rho$, the scale factor enumerated in Tab. 4.1, (2) the statistical uncertainty on the raw number of SM MC events that are subtracted from the SS sample

\footnotetext{
${ }^{1}$ For $\mathrm{NN}_{\tau}$ selections of $0.92 / 0.90 / 0.91$.
} 
to get the MJ yields, and (c) the statistical uncertainty of the SS event yields. Added in quadrature, the total is $5.0 \%$.

\subsubsection{Shape Systematics}

There are four systematics which are computed with shape uncertainties: Jet Reconstruction and Identification, Jet Energy Scale, Jet Energy Resolution, and Jet Vertex Confirmation. Altering theses four parameters can greatly effect the shape of jet-related distributions, as well as change which jets pass through specific jet requirements.

Jet Reco/ID - MC jets have a higher reconstruction/identification efficiency than jets from data, thus scale factors are applied to match the rates.

Jet Energy Scale and Resolution - MC jets are adjusted to match the energy spectrum and resolution of data jets.

Vertex Confirmation - Additional correction factors are applied to account for differences in tagged $\mathrm{MC}$ and data vertex confirmation rates. This requirement is only made for Run IIb data because the average instantaneous luminosity is twice as large in Run IIb and the jet fake rate is therefore much higher.

MC signals and SM MC backgrounds are generated with each parameter sequentially shifted down by $-1 \sigma$. A new BDT output is derived in both the $\mathrm{T}$ and $\mathrm{W}$ subsamples. The difference from the nominal distribution is given by:

$$
\delta \mathrm{BDT}_{-1 \sigma}=\frac{\mathrm{BDT}_{\text {nominal }}-\mathrm{BDT}_{-1 \sigma}}{\mathrm{BDT}_{\text {nominal }}}
$$

where $\mathrm{BDT}_{\text {nominal }}$ is the original BDT, and $\mathrm{BDT}_{-1 \sigma}$ is the adjusted BDT.

Various simplifications are made to expedite this calculation. First, it is observed that the magnitudes of $\pm 1 \sigma$ variations are nearly identical so symmetric un- 
certainties are used based on the $-1 \sigma$ values. Second only the $m_{H}=115 \mathrm{GeV}$ signals are used and applied to all other mass points. Although the shape of the uncertainty distributions change slightly if signals from different masses are used, previous studies showed that the overall limit is unaffected. This is because this search is largely statistics limited. Third, only the Run IIb MC is used because it has the highest statistics. Also, to avoid unnecessary statistical fluctuations, the bins of the shape systematics have been coarsened. Finally, because the MJ contribution is small, the uncertainty in the MJ shape is not considered.

The fractional uncertainty in BDT output relative to the nominal for signal, $Z+$ $j$, and $W+j / t \bar{t}$ is shown in the Fig. 6.1 for jet identification. ${ }^{2}$ The top row corresponds to the $\mathrm{T}$ subsample, and the bottom row to the $\mathrm{W}$ subsample. When considering these plots care must be taken to identify the largest contributing factors. In the T subsample the $Z+$ jets (top middle) background is dominant so changes to this background clearly effect the final discriminant more than the secondary backgrounds. Similarly, in the $\mathrm{W}$ subsample $W+$ jets is the dominant background (lower right). Typically the most variation happens for BDT values less than zero because the majority of the backgrounds are contained in this region. The highest bin in the $\mathrm{W}$ subsample $W+$ jets occurs at zero because most of this background is centered there.

\subsubsection{Incorporating Systematic Uncertainties}

The aforementioned systematics are incorporated into this analysis by the limit setting program COLLIE [33]. COLLIE attempts to find the best fit for data by varying all the systematic uncertainties found in Tab. 6.1 simultaneously. Figure 6.2 (a) shows the values of $\sigma$ for each individual systematic needed to achieve the best fit. All the values tend to be negative since there is a slight excess of background over

\footnotetext{
${ }^{2}$ The other shape systematics are shown in Appendix C.
} 
Table 6.1. Systematic uncertainties in percent. The range of jet shape uncertainties is shown separately for the $\mathrm{T}$ and $\mathrm{W}$ subsamples.

\begin{tabular}{c|c|c}
\hline \hline Source & type & Uncertainty (\%) \\
\hline Luminosity (DØ specific) & flat & 4.1 \\
Luminosity (Tevatron common) & flat & 4.6 \\
$e$ ID, track match, iso. & flat & 4 \\
$e$ trigger & flat & 2 \\
$\tau$ energy correction & flat & 9.8 \\
$\tau$ track efficiency & flat & 1.4 \\
$\tau$ selection by type & flat & $5.5,4.0,6$ \\
$W / Z+$ light flavor XS & flat & 6.0 \\
$t \bar{t}$, single top XS & flat & 7.0 \\
diboson XS & flat & 6.0 \\
$V H$ signal XS & flat & 6.2 \\
VBF signal XS & flat & 4.9 \\
GGF signal XS normalization & flat & 33 \\
GGF signal XS PDF & flat & 29 \\
jet vetex confirmation & shape & $4-20(\mathrm{~T}) 2-15(\mathrm{~W})$ \\
Jet ID/reco eff. & shape & $4-20(\mathrm{~T}) 2-15(\mathrm{~W})$ \\
Jet $E$ resolution. & shape & $5-19(\mathrm{~T}) 2-15(\mathrm{~W})$ \\
JES & shape & $4-20(\mathrm{~T}) 2-15(\mathrm{~W})$ \\
PDF & flat & $1.6(\mathrm{sig}), 2.0(\mathrm{bknd})$ \\
MJ normalization & flat & 5.0 \\
\hline \hline
\end{tabular}

data in the final sample. To gauge how individual systematics can effect the final result the N-1 test is considered, where the change to the best fit curve, quantified by $\Delta \chi^{2}$, is computed when each systematic is removed individually. Figure 6.2 (b) shows the value of $\Delta \chi^{2}$ for each systematic. The tau energy scale has the largest effect on the fit, but this is only a small overall change. Finally, Fig. 6.2 (c) shows the total systematic uncertainty per bin of the BDT output. The blue line shows the uncertainty taken from the central value fitting when they systematics are not allowed to float. When allowing for the best fit to background only, the green line is achieved. Since there is comparatively a very small amount of signal present, the signal plus background fit (red line) is nearly identical to the background only fit in all bins except the highest. The small systematic uncertainty, $\sim 2 \%$ in the BDT 
region greater than zero shows that this analysis is clearly statistics limited and that the systematic uncertainties have only a small effect.

COLLIE has two primary modes of calculating limits: CLFast and CLFit2. In the CLFast method no systematic uncertainties are considered, while in CLFit2 the full systematic calculations are applied. The ratio of these two results can show how influential the systematic uncertainties are on the final result. In this analysis the degradation is only about $20 \%$ showing again that it is statistics limited rather than systematics limited (see Section 6.2.4).

\subsection{Limit Setting}

\subsubsection{Overview}

In the case when there is no clear excess of data over background a modified frequentist method is used to set upper limits on the SM Higgs boson cross section. Two hypotheses are used, signal + background, called the test hypothesis, and background only, called the null hypothesis. 50,000 "pseudo-experiments" are used to convert the test statistic, the log-likelihood ratio (LLR), into a confidence level (C.L.) limit. In each pseudo-experiment the number of signal and background events in each bin of the final discriminant are varied according to Poisson statistics. The confidence level is defined as:

$$
C L_{s}=\frac{1-C L_{s+b}}{C L_{b}}
$$

where $C L_{s+b}\left(C L_{b}\right)$ is the probability that each pseudo-experiment is less signal-like than observed for the signal + background (background only) hypothesis. Traditionally in Higgs searches, cross sections are excluded at a $95 \% C L_{s}$. This means there is 
a $95 \%$ chance that the observed events agree with a background only hypothesis up to a certain factor.

To develop the C.L. a test statistic is used. The log-likelihood ratio (LLR) is equal to

$$
L L R=-2 \ln Q=2\left[s_{i}-d_{i} \ln \left(1+\frac{s_{i}}{b_{i}}\right)\right]
$$

where $Q$ is the ratio of the probability that the data is consistent with signal + background to background only

$$
Q=\prod_{i=1}^{N_{\text {bins }}}\left(\frac{e^{-s_{i}+b_{i}}\left(s_{i}+b_{i}\right)^{d_{i}}}{d_{i} !} / \frac{e^{-b_{i}}\left(b_{i}\right)^{d_{i}}}{d_{i} !}\right)
$$

where $s_{i}$ is the signal estimation, $b_{i}$ is the background estimation, and $d_{i}$ is the result of the pseudo-experiment. The ratio is computed in each bin of the final discriminant from 1 to $N_{b i n s}$, the total number of bins in the final discriminant, the 15 BDTs in Figs. $5.15-5.24$.

The systematic uncertainties are incorporated as nuisance parameters to the LLR. In each pseudo-experiment systematic uncertainties are included for both signal and background by using Gaussian smearing of Poisson probabilities.

\subsubsection{Log-Liklihood Ratios}

The LLRs for the $\mathrm{T}$ and $\mathrm{W}$ subsamples are shown separately and combined in Fig. 6.3. The expected distributions for $s+b$ and $b$-only are plotted in the red and black dashed lines. The greater the difference between these two lines the more power the channel has to discriminate $s+b$ from $b$-only. Clearly in the T subsample (Fig. 6.3 (a)) there is more separation, which is a reflection of the better signal significance in the $\mathrm{T}$ subsample. The green and yellow bands represent the $\pm 1 \sigma$ and $\pm 2 \sigma$ uncertainties on 
the background and provide an estimate for how sensitive the channel is to signal-like background fluctuations in the data. The W subsample (Fig. 6.3 (b)) has quite small bands but hardly any $s+b$ to $b$-only separation. The solid black line represents the observed LLR distribution from data. If this line follows the $\mathrm{LLR}_{S+B}$ curve more closely than the $\mathrm{LLR}_{B}$ curve then the data are said to be more signal-like. As long as the $s+b$ curve is within the $\pm 2 \sigma$ band any data that agree with this curve are still also consistent with the $b$-only prediction. If the data agree with the $s+b$ prediction and are outside the $\pm 2 \sigma$ band the possibility exists to claim evidence or discovery of a new particle. The accepted criteria for evidence is data with $3 \sigma$ excess over background, and for discovery the requirement is above $5 \sigma$.

Examining the combined $\mathrm{T}$ and W LLR in Fig. 6.3 (c) a small separation of $\operatorname{LLR}_{B}$ and $\operatorname{LLR}_{S+B}$ is apparent at low $m_{H}$. The LLR $O B S$ line is entirely contained within the $1 \sigma$ band and thus offers no clear presence of signal-like data. It is important to note the smoothness of the combined LLR distribution and contrast it to Figure 5.13 (b) and (d).

\subsubsection{Cross Section Limits}

Another formulation of the results is found in Tab. 6.2 which lists the factors by which the Higgs cross section times branching ratio would need to be multiplied in order to exclude it at 95\% C.L. for the $\mathrm{T}$ and $\mathrm{W}$ subsample separately and combined and also without (CLFast) and with (CLFit2) the systematic uncertainties. When the background prediction is used in Eq. 6.4 the confidence level is $C L^{e x p}$, called the expected limit, and when the data are used the confidence level is $C L^{\text {obs }}$, or the observed limit. The Higgs may be excluded by any search channel only if the expected and observed limits are less than unity. If the expected limit is less than unity and 
the observed limit is more than $3 \sigma$ above the background the data are said to suggest evidence of the Higgs boson. No such excess is found here.

In Fig. 6.4 (a) the T subsample shows a flat expected limit for $105 \leq m_{H} \leq 125$ $\mathrm{GeV}$, above which the limit increases due to the drop-off of the $H \rightarrow \tau \tau$ cross section. Across the full mass region the observed limit agrees well with the expected limit. The W subsample limit (Fig. 6.4 (b)) reflects the cross section of $H \rightarrow W W$ since its strongest limit is set above $m_{H}=130 \mathrm{GeV}$ and increases as $m_{H}$ decreases. There is an excess in the observed limit at low $m_{H}$, however, since the $\mathrm{W}$ subsample limit is much weaker in this mass region it does not bias the combined $\mathrm{T}+\mathrm{W}$ result. Figure 6.4 (c) shows the combined $\mathrm{T}$ and $\mathrm{W}$ subsample limit which is very flat across most of the mass range and the observed limit closely follows the expected.

Table 6.2. Ratio of the expected and observed 95\% C.L. limits to the SM expectations.

\begin{tabular}{c|cc|cc|cc|cc|cc|cc}
\hline \hline & \multicolumn{9}{|c|}{ CLfast } & \multicolumn{3}{c|}{ CLfit2 } \\
\hline & \multicolumn{2}{|c|}{ T } & \multicolumn{2}{c|}{ W } & \multicolumn{2}{c|}{ T+W } & \multicolumn{2}{c}{$\mathrm{T}$} & \multicolumn{2}{c}{$\mathrm{W}$} & \multicolumn{2}{c}{$\mathrm{T}+\mathrm{W}$} \\
mass & exp. & obs. & exp. & obs. & exp. & obs. & exp. & obs. & exp. & obs. & exp. & obs \\
\hline 105 & 13.0 & 6.8 & 54.0 & 94.4 & 12.5 & 7.3 & 16.3 & 14.6 & 62.3 & 107 & 14.9 & 11.8 \\
110 & 12.6 & 6.9 & 55.0 & 91.1 & 12.2 & 7.5 & 15.6 & 14.6 & 64.3 & 101 & 14.4 & 12.5 \\
115 & 12.4 & 7.0 & 56.0 & 85.8 & 12.0 & 7.5 & 14.9 & 14.8 & 66.5 & 93.3 & 14.2 & 11.7 \\
120 & 13.3 & 9.2 & 46.2 & 67.8 & 12.5 & 9.6 & 15.7 & 19.7 & 56.6 & 75.4 & 14.5 & 15.3 \\
125 & 13.6 & 9.1 & 38.6 & 59.5 & 12.6 & 9.7 & 15.9 & 18.7 & 46.1 & 65.0 & 14.6 & 16.0 \\
130 & 16.7 & 10.0 & 38.9 & 56.1 & 15.2 & 11.5 & 20.1 & 21.8 & 51.5 & 65.3 & 18.3 & 19.5 \\
135 & 19.1 & 11.5 & 36.5 & 45.8 & 16.7 & 12.3 & 23.6 & 25.6 & 51.0 & 51.8 & 20.4 & 21.4 \\
140 & 25.9 & 15.5 & 30.4 & 36.8 & 19.0 & 14.7 & 31.0 & 33.9 & 40.0 & 40.8 & 24.4 & 26.3 \\
145 & 33.3 & 19.9 & 30.8 & 37.8 & 21.8 & 18.2 & 42.5 & 47.6 & 45.5 & 44.0 & 29.8 & 36.0 \\
150 & 47.2 & 29.1 & 21.7 & 24.9 & 19.2 & 17.7 & 58.4 & 69.5 & 29.0 & 27.5 & 24.8 & 30.8 \\
\hline \hline
\end{tabular}




\subsubsection{Systematic Effects}

As previously mentioned this search is statistics limited, in part this can be seen by observing the change in limits and LLR when using CLFast and CLFit2. Figure 6.5 (a) and (b) show the limit and LLR as calculated by CLFast (without systematics). The observed limit is consistently under the expected limit due to the excess of MC as compared to data. Allowing for systematic uncertainties brings the observed limit into better agreement with the expected with an overall degradation of about $20 \%$ which is low compared to others systematics dominated searches which can see degradations up to $40 \%$. Similarly in the LLR distribution, incorporating the systematics has brought the observed LLR into better agreement with the background prediction. 
Signals 115 jid systematics

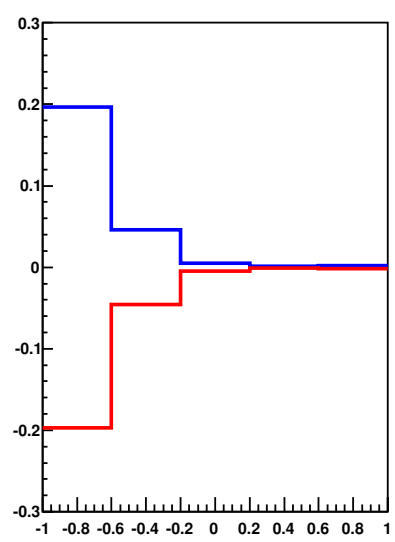

Signals 115 jid systematics

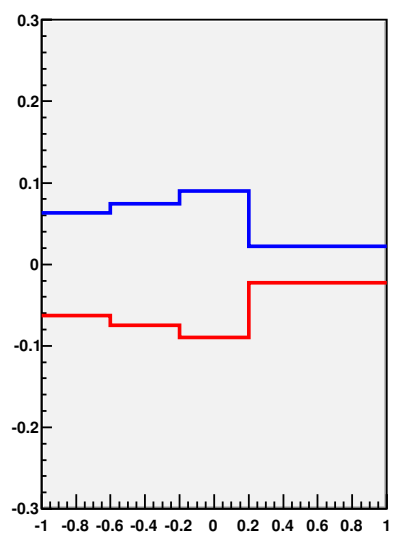

Zjets 115 jid systematics

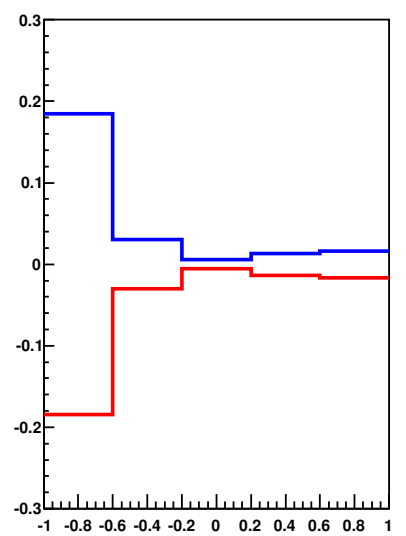

Zjets 115 jid systematics

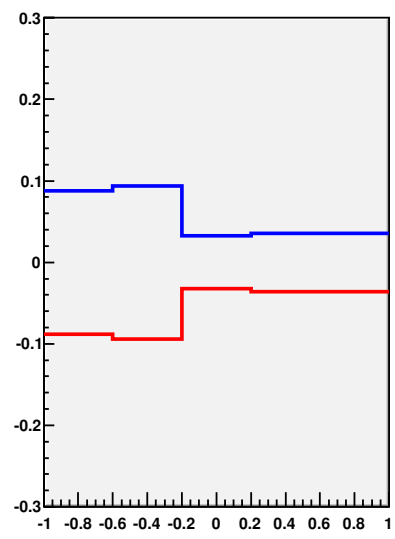

Wjets+tt 115 jid systematics

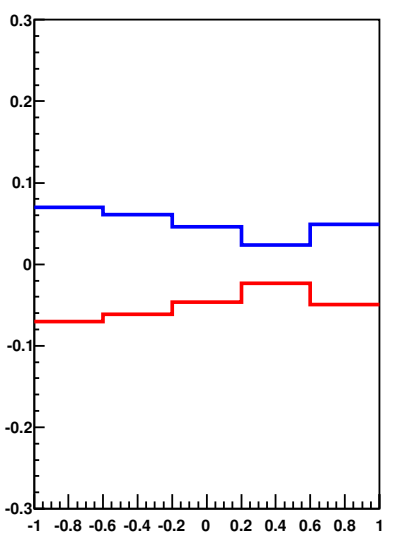

Wjets+tt 115 jid systematics

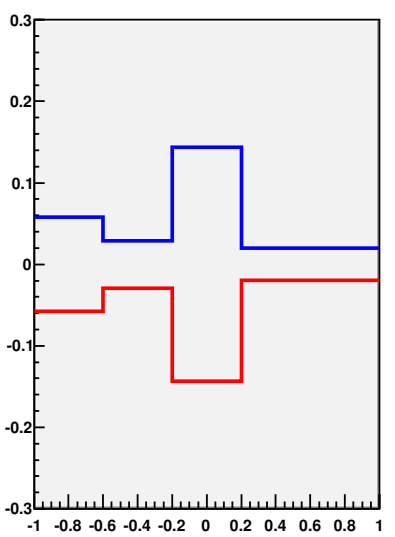

Figure 6.1. Normalized $\delta \mathrm{BDT}_{ \pm 1 \sigma}$ outputs of the jet ID and reconstruction efficiencies, for (top row) the $\mathrm{T}$ subsample at $115 \mathrm{GeV}$ and for (bottom row) the $\mathrm{W}$ subsample at $115 \mathrm{GeV}$. The signals, $Z+$ jets and $W+$ jets $+t \bar{t}$ backgrounds are shown in the left, middle and right columns respectively. 


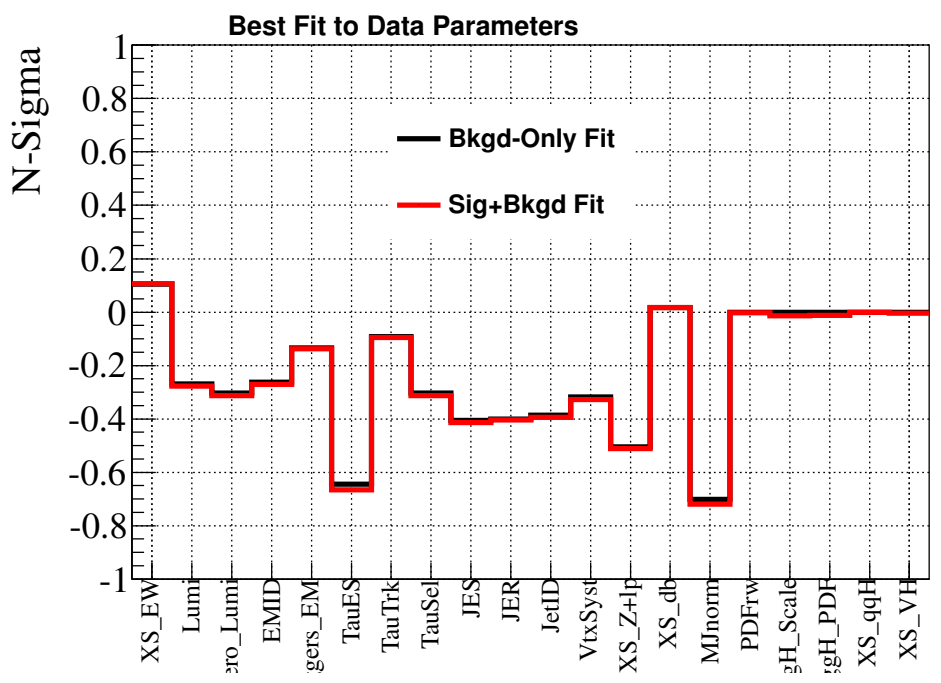

(a)

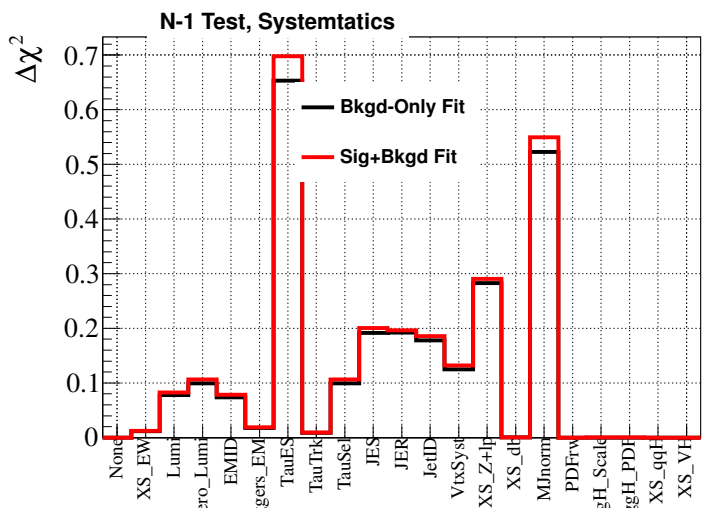

(b)

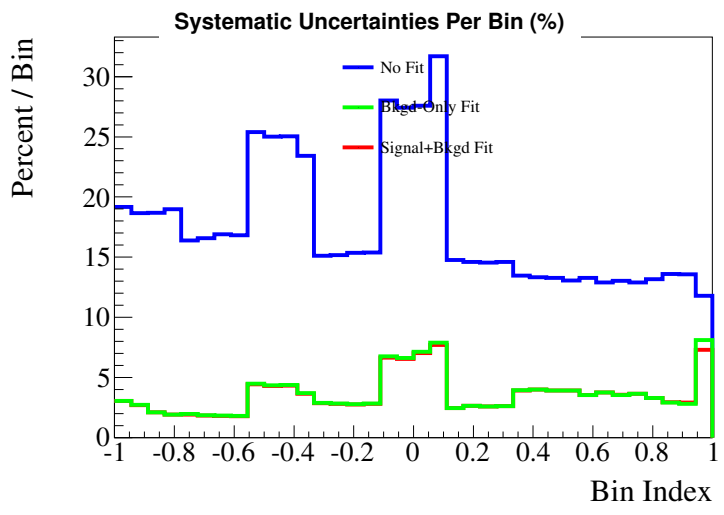

(c)

Figure 6.2. Various plots to quantify the effect of systematic uncertainties. (a) $\sigma$ value to achieve the best fit of background to data, (b) effect of removing any single uncertainty on the best fit parameter $\Delta \chi^{2}$ and (c) the effect of the total uncertainty by BDT output bin. 


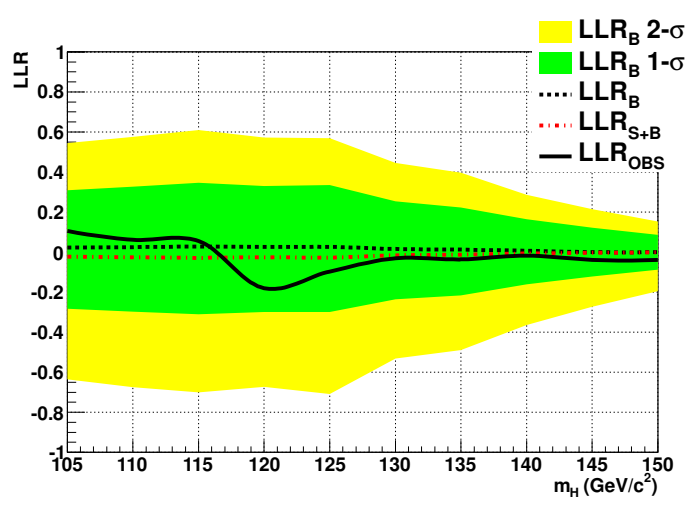

(a)

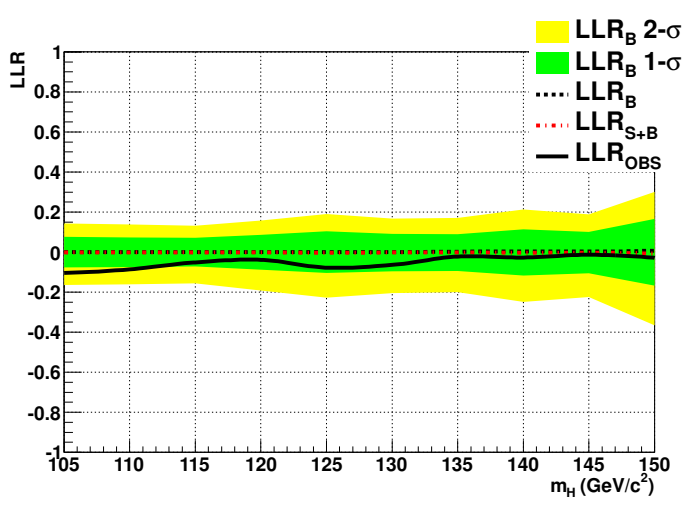

(b)

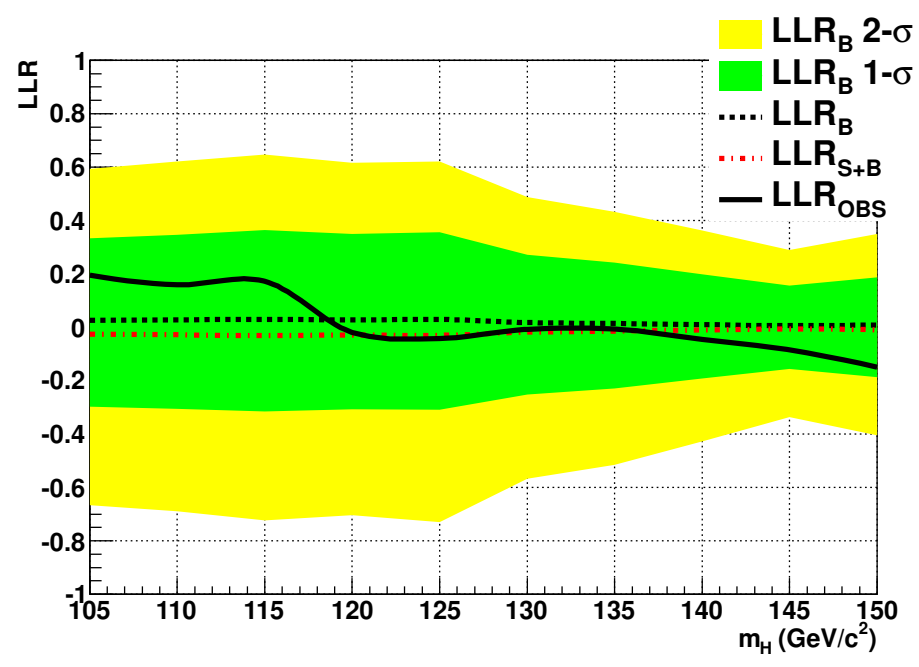

(c)

Figure 6.3. LLR for (a) the $\mathrm{T}$ subsample, (b) the $\mathrm{W}$ subsample and (c) the $\mathrm{T}$ and W subsamples combined as a function of Higgs mass, for expected background only (black dotted line), expected signal + background (red dotted line) hypotheses, and the observed values (solid black line). The $\pm 1 \sigma$ and $\pm 2 \sigma$ variations from the expected background only hypothesis are shown in green and yellow bands respectively. 


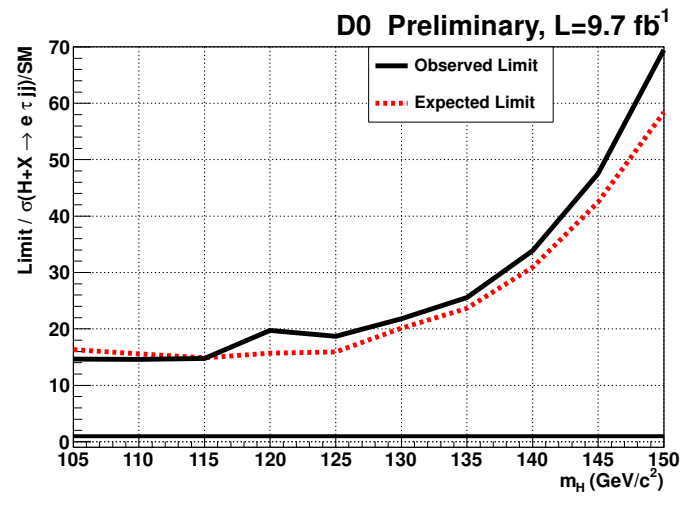

(a)

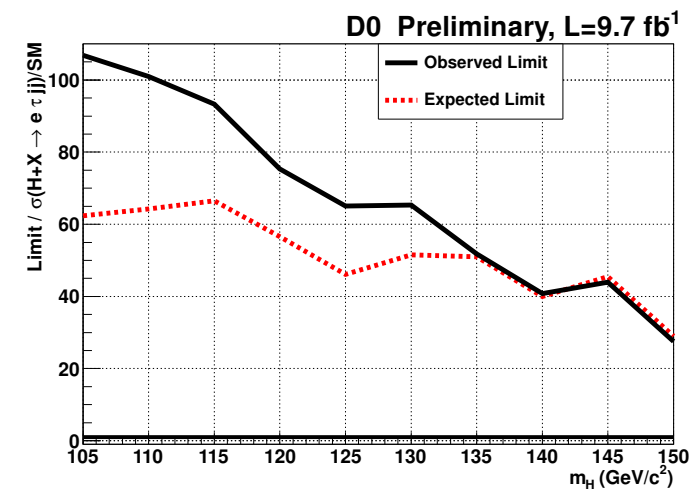

(b)

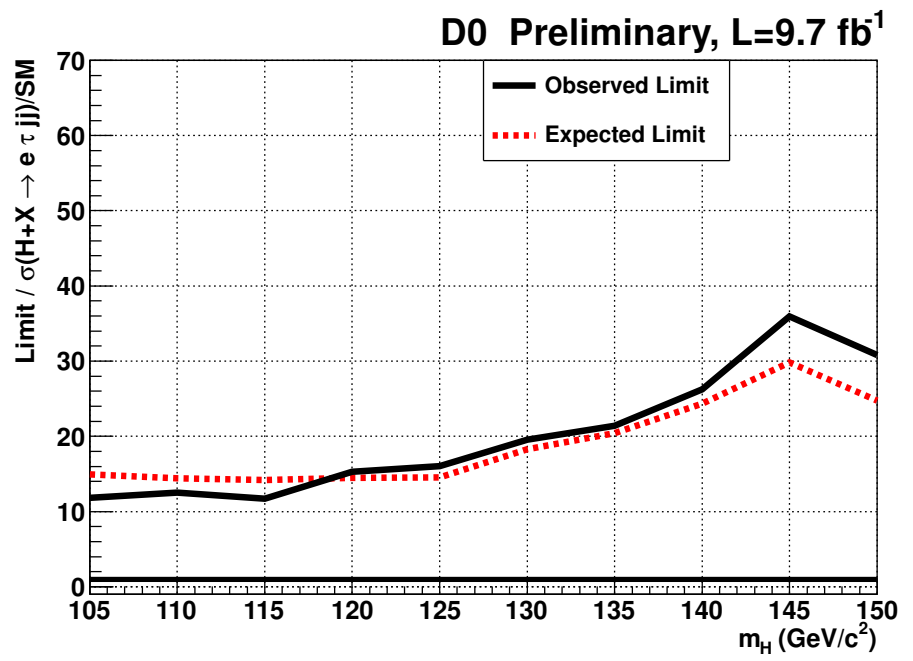

(c)

Figure 6.4. Cross section limits for (a) the T subsample, (b) the W subsample and (c) the $\mathrm{T}$ and $\mathrm{W}$ subsamples combined as a function of Higgs mass, for expected background only (dashed red line), and the observed values (black line). 


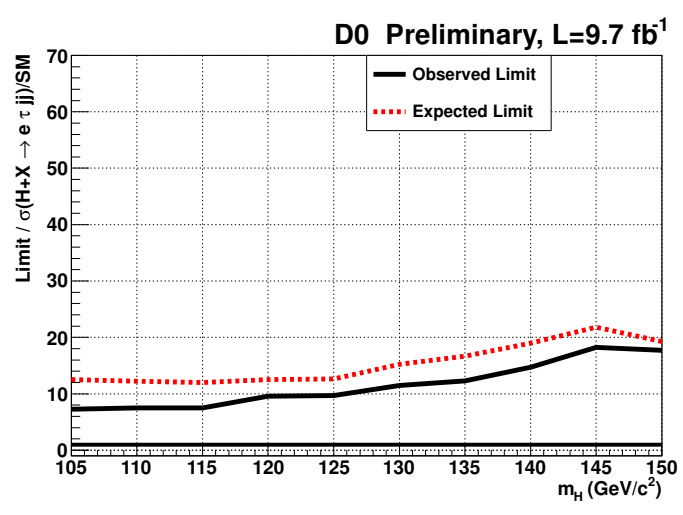

(a)

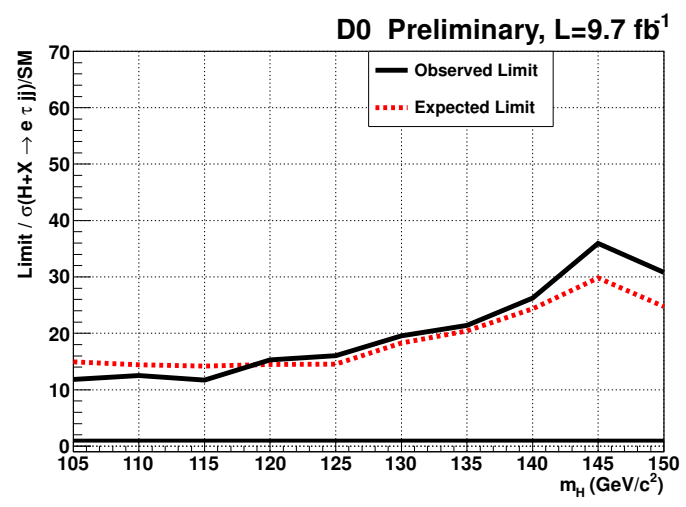

(c)

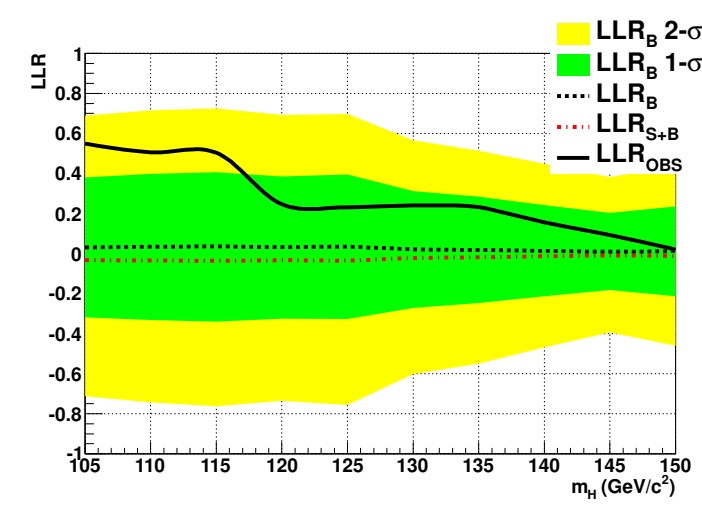

(b)

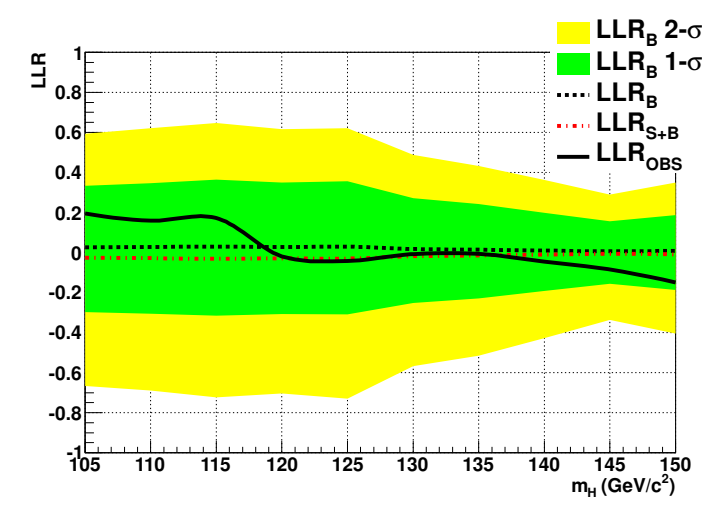

(d)

Figure 6.5. Cross section limits and LLR distributions for (a), (b) the combined subsamples without systematic uncertainties; and (c), (d) the combined subsamples with the systematic uncertainties as a function of Higgs mass. 


\section{CHAPTER 7}

\section{CONCLUSIONS}

Individual Higgs search channels at the Tevatron rarely have exclusion or discovery sensitivity by themselves. Therefore combinations of analyses over multiple channels are used to achieve the maximum overall sensitivity. This analysis was performed in collaboration with the channel $H \rightarrow \mu \tau_{h}(\mu \tau j j)$, a combination referred to as the $\ell \tau j j$ channel which dominate the $H \rightarrow \tau \tau$ sensitivity at $\mathrm{D} \varnothing$ and the Tevatron. Additionally the $\ell \tau j j$ channel is combined with all other D $\varnothing$ and CDF channels to produce the Tevatron Combination. With this combination CDF and DØ were able to make significant Higgs exclusions and provided evidence for a new boson decaying into a $b \bar{b}$ quark pair [32].

\subsection{Combinations}

\subsection{1 $\mu \tau j j$ and $e \tau j j$}

The $\mu \tau j j$ channel is, in general, more sensitive than the $e \tau j j$ channel largely due to the composition of the backgrounds. The selections for all the final state objects were the same except the electron was replaced with a muon. It is much easier to select isolated muons, than isolated electrons, hence the $Z \rightarrow \mu \mu$ background of the

$\mu \tau j j$ channel is proportionally much less than the $Z \rightarrow e e$ background of the $e \tau j j$ channel.

The $\mu \tau j j$ channel also follows the same multivariate approached used here. The maximum sensitivity for combined the $\mu \tau j j$ and $e \tau j j$ analysis is achieved when all four subsamples are combined. Table 7.1 shows the combined $\mu \tau j j$ and $e \tau j j$ 
limits for the $\mathrm{T}$ and $\mathrm{W}$ subsamples and for the $\mathrm{T}+\mathrm{W}$ combination. Figure 7.1 shows the expected and observed limits for the $\mathrm{T}$ and $\mathrm{W}$ subsamples. Each limit loosely resembles the subsample limits for the $e \tau j j$ channel since the $\mu \tau j j$ limits have a similar shape and sensitivity. The combined $\mu \tau j j+e \tau j j$ limit plot is shown in Fig. 7.2 with the $\pm 1 \sigma$ and $\pm 2 \sigma$ bands. No significant excess of data is found, however, the limit has improved by $\sim 20 \%$ over the previous version of this analysis. This result is also the most stringent limit including $H \rightarrow \tau \tau$ ever completed at the Tevatron. The expected limit is essentially flat from $105 \leq m_{H} \leq 150 \mathrm{GeV}$, a rare feature among Higgs searches at the Tevatron, owing to its use of two decay modes, $H \rightarrow \tau \tau$ and $H \rightarrow W W$.

Table 7.1. Final limits obtained for the combined Run II $\mu \tau j j$ and $e \tau j j$ analysis.

\begin{tabular}{c|cc|cc|cc}
\hline \hline Higgs mass & \multicolumn{2}{|c|}{$\mathrm{T}$} & \multicolumn{2}{c|}{$\mathrm{W}$} & \multicolumn{2}{c}{$\mathrm{T}+\mathrm{W}$} \\
$(\mathrm{GeV})$ & exp. & obs. & exp. & obs. & exp. & obs. \\
\hline 105 & 10.3 & 11.8 & 50.9 & 69.3 & 9.4 & 9.4 \\
110 & 9.9 & 11.7 & 52.6 & 74.1 & 9.1 & 9.8 \\
115 & 9.5 & 11.2 & 48.0 & 73.5 & 9.0 & 9.5 \\
120 & 10.1 & 13.3 & 36.4 & 68.8 & 9.4 & 11.1 \\
125 & 10.4 & 12.8 & 28.4 & 53.3 & 9.0 & 11.3 \\
130 & 12.4 & 14.1 & 23.5 & 40.8 & 10.2 & 11.8 \\
135 & 13.6 & 15.9 & 21.3 & 36.6 & 10.8 & 13.5 \\
140 & 17.6 & 19.1 & 16.8 & 31.4 & 11.5 & 16.0 \\
145 & 23.5 & 27.6 & 14.7 & 27.9 & 11.5 & 17.9 \\
150 & 29.9 & 33.3 & 12.9 & 23.7 & 11.1 & 17.2 \\
\hline \hline
\end{tabular}

The LLR distributions for the combined $\mu \tau j j+e \tau j j$ are shown in Fig. 7.3.

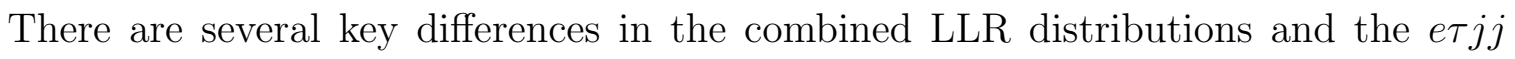
distributions of Fig. 6.3. In the T subsample (Fig. 7.3 (a)), there is a much greater separation between the $s+b$ hypothesis (red dashed line) and the $b$-only hypothesis 


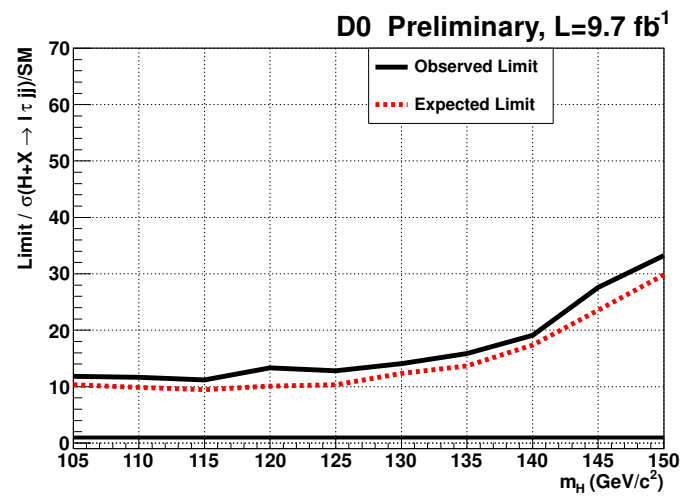

(a)

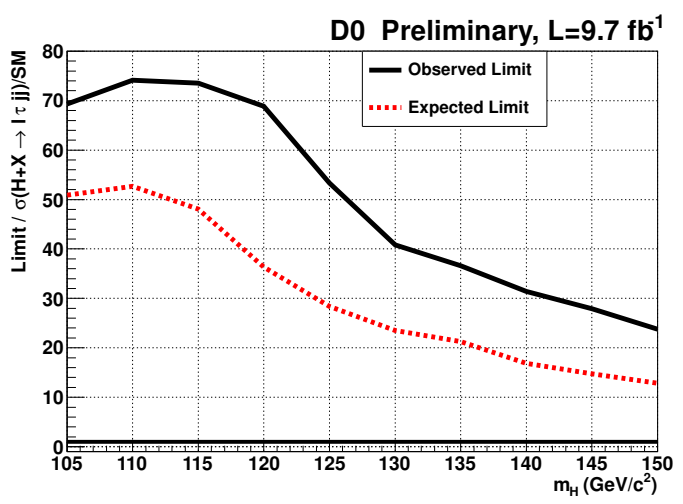

(b)

Figure 7.1. Limits for the combined $\mu \tau j j$ and $e \tau j j$ analysis for (a) the T subsample and (b) the W subsample as a function of Higgs mass, for expected background only (red dotted line), and the observed values (solid black line).

(black dashed line) which shows that combining the $\mu \tau j j$ and $e \tau j j$ increases the sensitivity above either search alone. The W subsample (Fig. 7.3 (b)) has larger uncertainty bands which arise from the $\mu \tau j j$ analysis which has more sensitivity in the $\mathrm{W}$ subsample for $m_{H}>135 \mathrm{GeV}$. The observed LLR shown in the combined $\mathrm{T}+\mathrm{W}$ distribution of Fig. 7.4 smoothly varies within the $\pm 1 \sigma$ band and has a small excursion into the $-2 \sigma$ band at $m_{H}=145 \mathrm{GeV}$, which is driven by the $\mu \tau j j \mathrm{~W}$ subsample. Overall the observed LLR statistically agrees with either the $s+b$ or b-only hypothesis.

\subsubsection{DØ and CDF Combinations}

As mentioned in Chapter 1, the dominant decay of the Higgs at low mass is $H \rightarrow b \bar{b}$. This channel poses many challenges, particularly in handling the MJ background and secondary vertexing of $b$-jets. Regardless, it is among the most sensitive channels at $\mathrm{D} \varnothing$ and $\mathrm{CDF}$. The exclusive search for $H \rightarrow W W$ was able to achieve single channel exclusion of Higgs mass $147<m_{H}<180 \mathrm{GeV}$. The $H \rightarrow \gamma \gamma$ search is inherently different from the dominant searches previously mentioned. The 


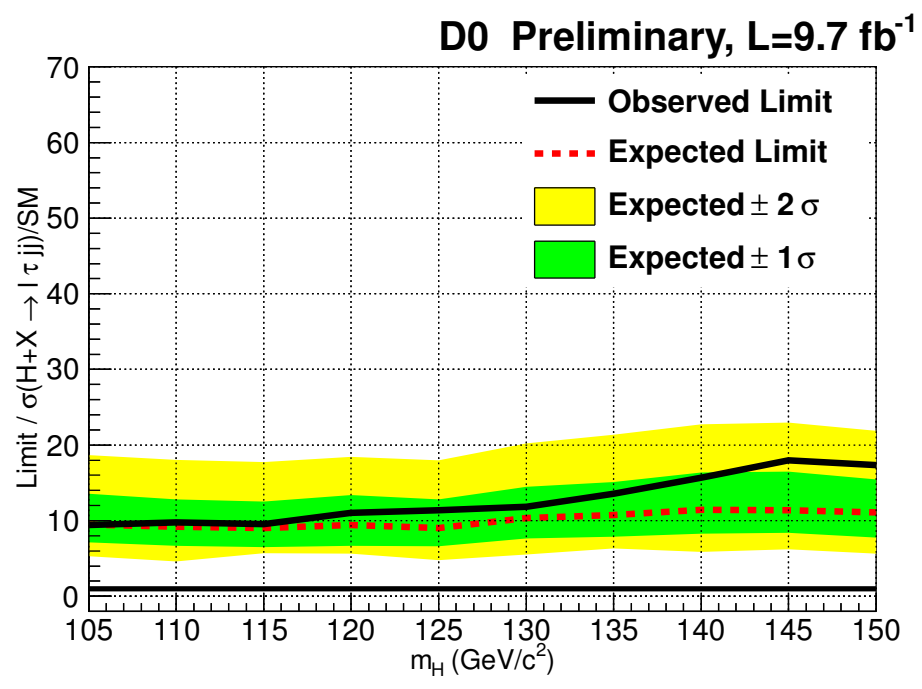

Figure 7.2. Limit plot for the combined $\mu \tau j j$ and $e \tau j j$ analysis as a function of Higgs mass. The relatively flat sensitivity can be attributed to the use of two Higgs decay modes.

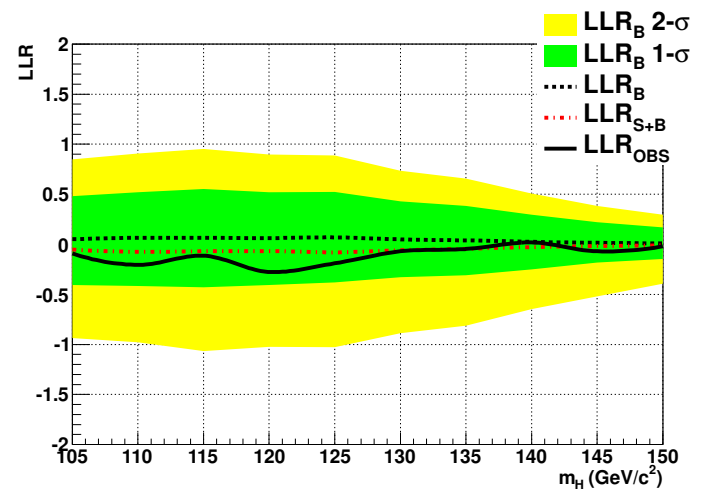

(a)

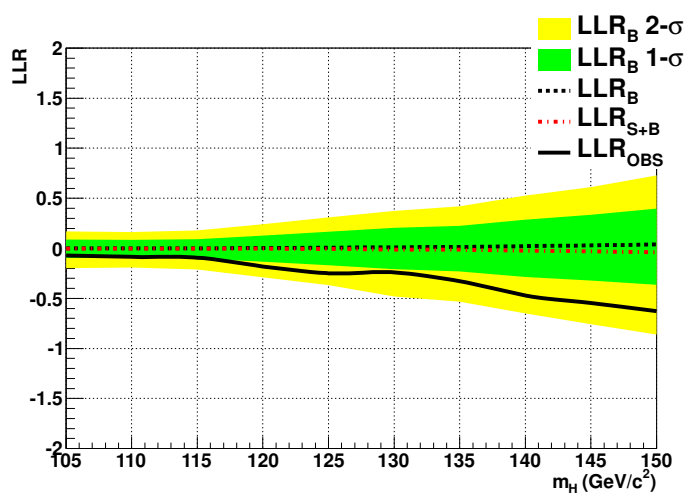

(b)

Figure 7.3. LLR for the combined $\mu \tau j j$ and $e \tau j j$ analysis for (a) the T subsample and (b) the W subsample as a function of Higgs mass, for expected background only (black dotted line), expected signal + background (red dotted line) hypotheses, and the observed values (solid black line). The $\pm 1 \sigma$ and $\pm 2 \sigma$ variations from the expected background only hypothesis are shown in green and yellow bands respectively. 


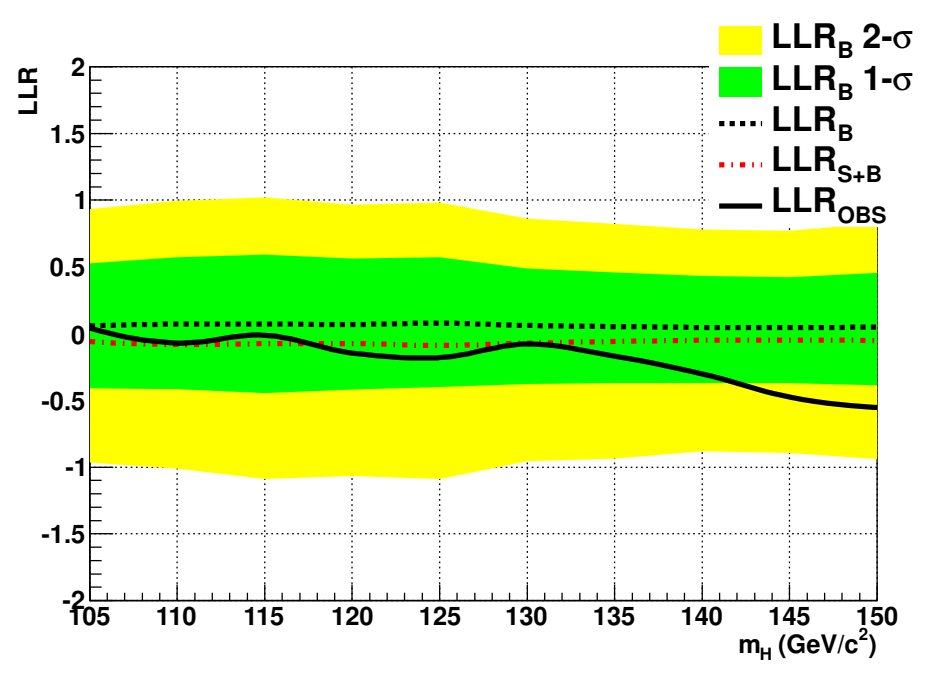

Figure 7.4. LLR for the combined $\mu \tau j j$ and $e \tau j j$ analysis as a function of Higgs mass, for expected background only (black dotted line), expected signal + background (red dotted line) hypotheses, and the observed values (solid black line). The $\pm 1 \sigma$ and $\pm 2 \sigma$ variations from the expected background only hypothesis are shown in green and yellow bands respectively.

cross section for $H \rightarrow \gamma \gamma$, is roughly three orders of magnitude less, however at the Tevatron (and LHC) the background is quite small which allows the channel to retain good sensitivity.

CDF and D $\varnothing$ combined these dominant channels with other SM Higgs searches to obtain maximal sensitivity. The limit plots for the individual channels are shown in Fig. 7.5. The high mass exclusion in the $W W$ channel is clear, and the expected exclusion for $m_{H}<115 \mathrm{GeV}$ is seen in the $b \bar{b}$ channel. The exclusion from $b \bar{b}$ is not as stringent as expected because of an excess of data above the predicted background at the level of $3.1 \sigma$. Similar to the $\ell \tau j j$ search the $\gamma \gamma$ channel has a fairly flat limit for $m_{H}<150 \mathrm{GeV}$, but does not achieve single channel exclusion.

The combined Tevatron limit plot is shown in Fig. 7.6. The Tevatron combination excludes the SM Higgs boson for $100<m_{H}<103 \mathrm{GeV}$ and $147<m_{H}<180$ GeV (green shaded). The primary high mass exclusion achieved by the Tevatron was 


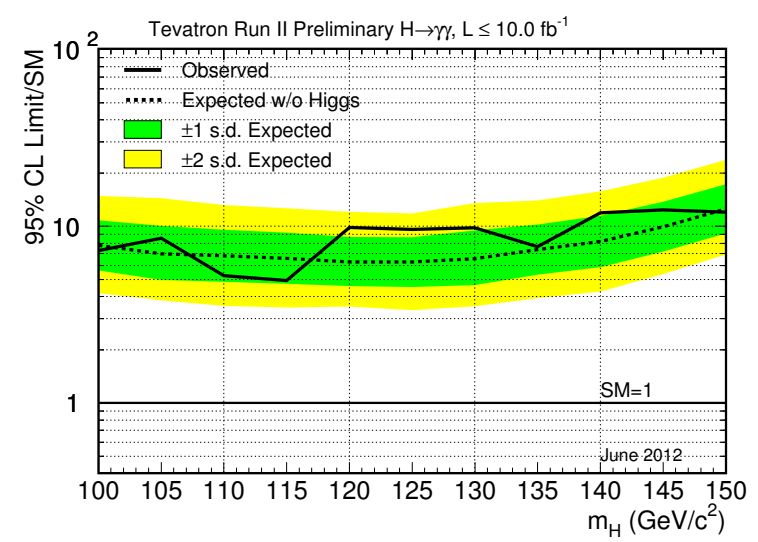

(a)

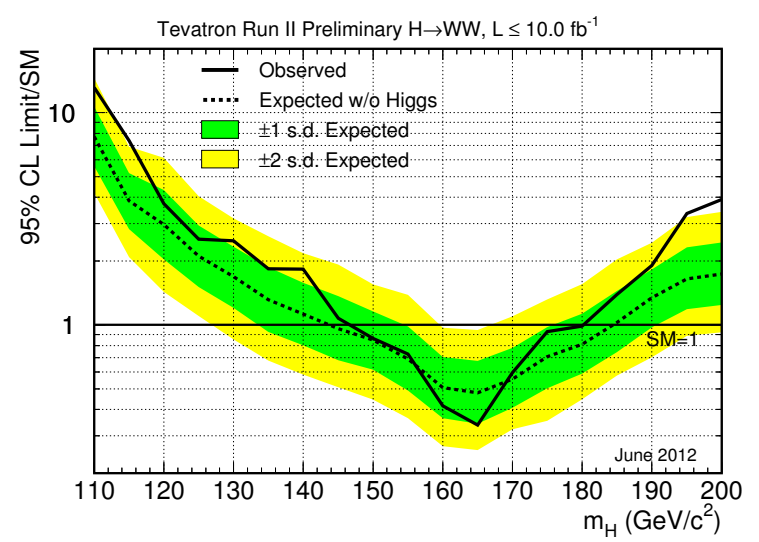

(b)

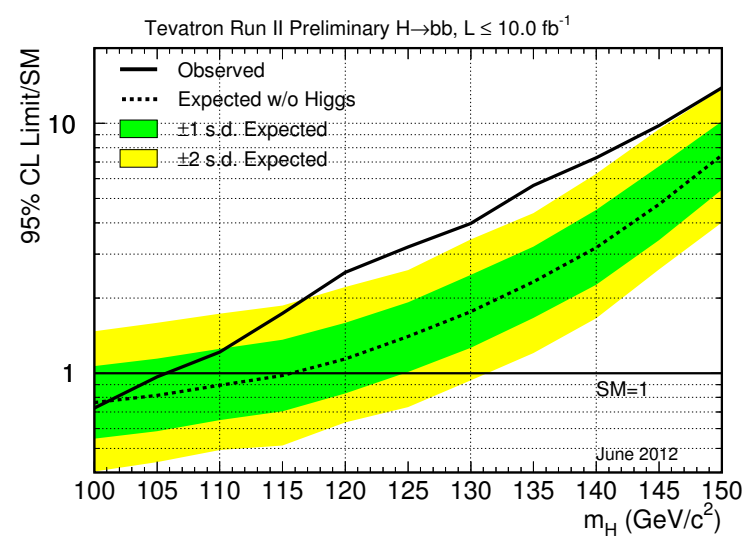

(c)

Figure 7.5. The combined CDF and D $\varnothing$ limits by channel: (a) $\gamma \gamma$, (b) $W W$ and (c) $b \bar{b}$. A broad excess is seen in the $b \bar{b}$ channel.

dominated by the $W W$ analyses. The expected exclusion at lower mass is not achieved because of a broad excess of data events above background from $115<m_{H}<140$ GeV. This excess is dominated by the $H \rightarrow b \bar{b}$ channel consistent in both CDF and $\mathrm{D} \varnothing$.

The LLR for the Tevatron combination is shown in Fig. 7.7, where a clear separation of the signal + background and background only hypotheses is present. In the region of the excess, the observed LLR tends to follow the signal + background hypothesis with a significance above $3 \sigma$. This excess is interpreted as evidence of a 
new boson decaying into a $b \bar{b}$ pair and is consistent with the discovery at the LHC discussed in Appendix D. Aspects of this excess have persisted through the six year search and have continued to grown in significance as shown in Fig. 7.8. Figure 7.9 shows the best fit signal cross section strength for $m_{H}=125 \mathrm{GeV}$. This is the average cross section enhancement factor for the observed excess. The $H \rightarrow \tau \tau$ segment is driven by the $\ell \tau j j$ combination presented in Section 7.1.1.

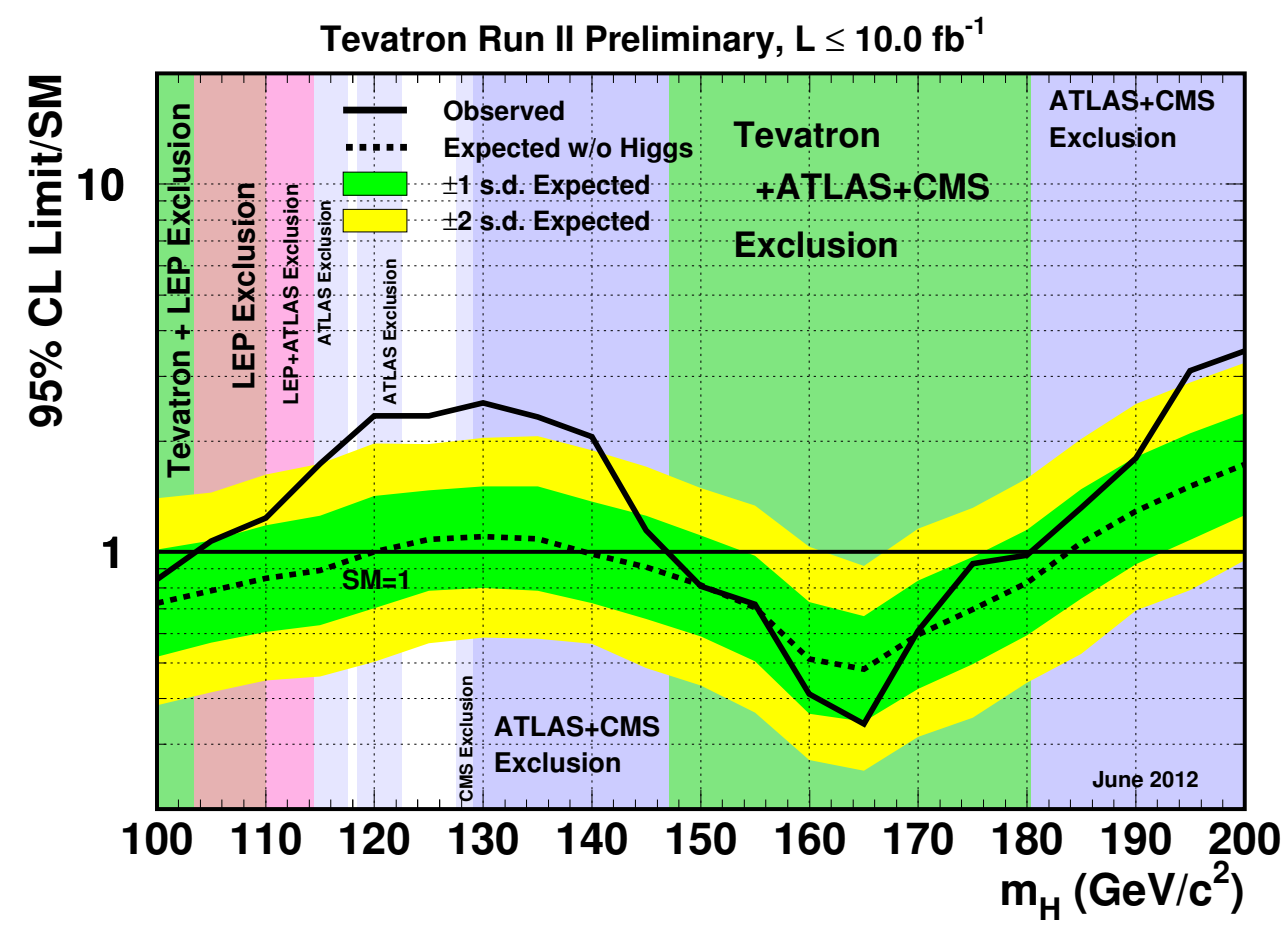

Figure 7.6. Higgs exclusion for the combined CDF and DØ experiments as a function of Higgs mass. The shaded regions indicate where the Higgs boson has been excluded by previous experiments. A significant excess in the region $115<m_{H}<140 \mathrm{GeV}$ gives evidence of a new boson. 


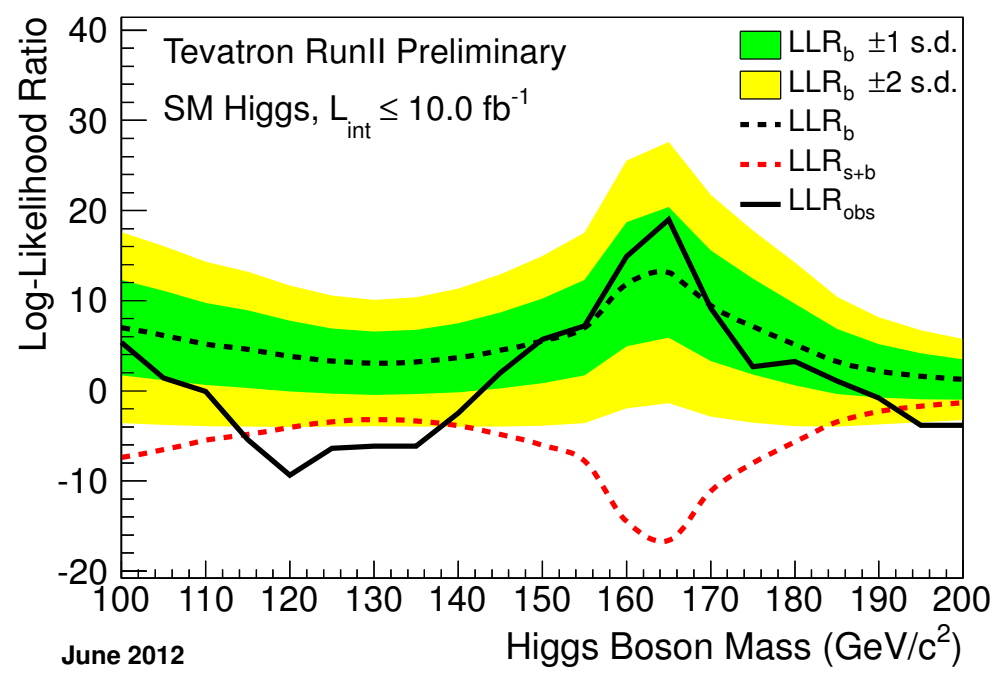

Figure 7.7. LLR for the combined CDF and DØ Experiments as a function of Higgs mass. In the region $115<m_{H}<140 \mathrm{GeV}$ the data agree well with the signal + background hypothesis.

\subsection{Discussion}

This analysis searched for the standard model Higgs boson decaying into one electron and one hadronic tau. Three production modes were considered, along with two decay modes: $H \rightarrow \tau \tau$ and $H \rightarrow W W$. Several improvements were implemented leading to a major increase in sensitivity over previous versions of the analysis. Improved algorithms increased the signal efficiency in tau object identification and to a greater degree increased both the signal efficiency and the background rejection of electron identification. Selection requirements were optimized to allow more events into the final discriminants. A new EJetsOR trigger was implemented that increased statistics and demonstrated good modeling. 17 new BDT variables were introduced, as well as the multivariate technique of creating two subsamples, enriched in $H \rightarrow \tau \tau$ and $H \rightarrow W W$.

A new Global BDT approach was created to combat the oscillatory nature of the LLR and limits found in previous multivariate approaches. The Global BDT 

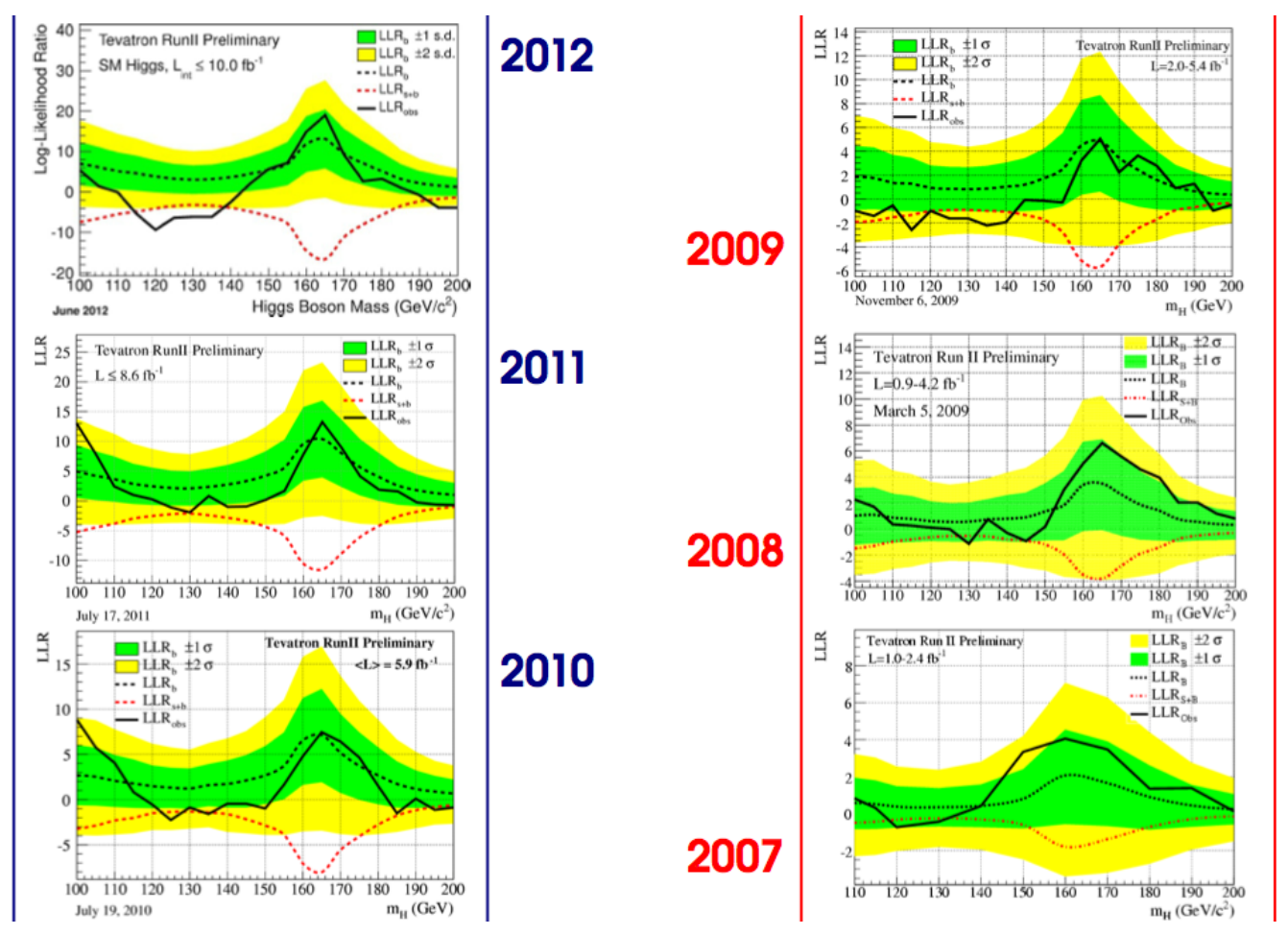

Figure 7.8. The Tevatron combined LLR plots from 2007-2012. In most plots the data follows the signal + background prediction in the region $110-140 \mathrm{GeV}$.

approach was found to significantly reduces the oscillatory behavior as compared to multivariate approaches that use separate trainings at each $m_{H}$. The continuity of the Global BDT results is much more desirable. A summary of all the major improvements completed during this analysis is found in Tab. 7.2.

Table 7.3 compares the expected and observed limits for the $4.3 \mathrm{fb}^{-1}$ result and the current version at each $m_{H}$. At $m_{H}=125 \mathrm{GeV}$ over $70 \%$ improvement in expected limits was achieved. The increase in luminosity from 4.3 to $9.7 \mathrm{fb}^{-1}$ only accounts for $30 \%$ of this improvement, the remainder came from increased background rejection during object identification, optimization of selection criteria, and improved multivariate techniques. Figure 7.10 shows clearly that the Global BDT method smooths the expected limit, and the impact of the various improvements. Finally, 


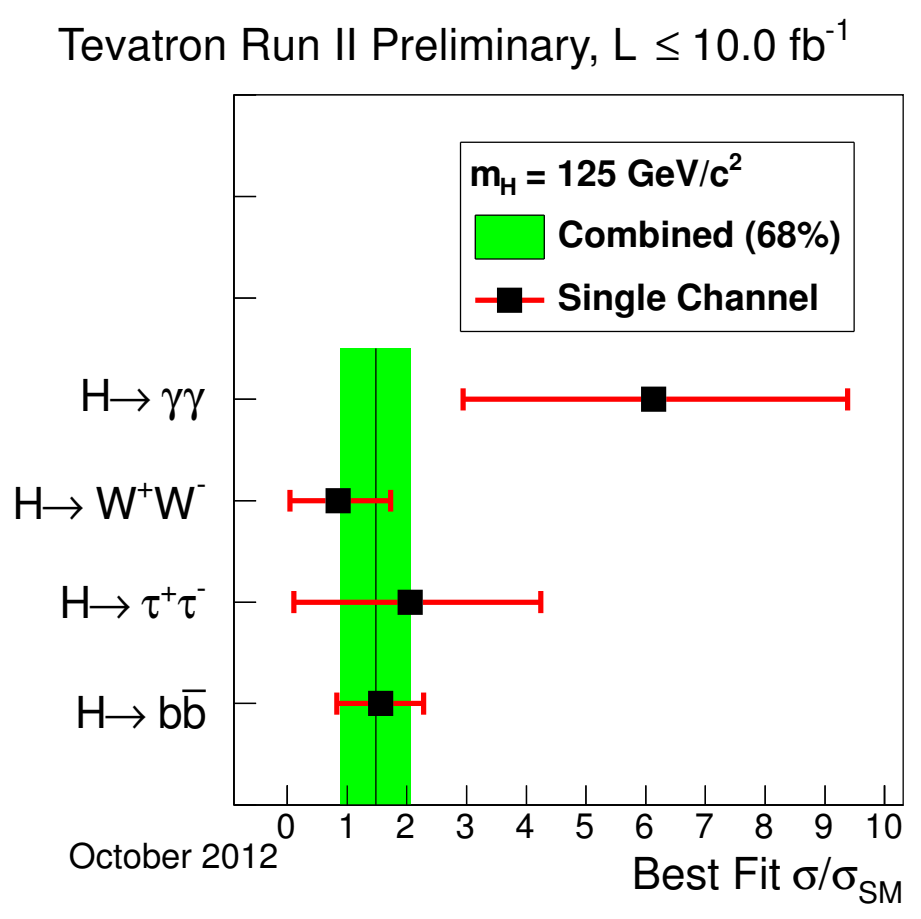

Figure 7.9. The best fit signal strength for the Tevatron combination (black line) and for each major decay mode (black squares). The green band is the $1 \sigma$ uncertainty on the average.

Fig. 7.11 compares the combined $e \tau j j$ and $\mu \tau j j$ limit to the previous version with the results presented here. There is roughly a $20 \%$ improvement in expected limits. Here again, the smooth distribution as a function of $m_{H}$ is a result of the Global BDT method.

\subsection{Final Summary}

No evidence for the SM Higgs boson was found. Upper limits on the cross section ratio at $95 \%$ C.L. were found to be 14.6 expected and 16.0 observed at $m_{H}=125$ $\mathrm{GeV}$. The e $\tau j j$ analysis was combined with its companion channel, $\mu \tau j j$. This limit was found to be 9.0 expected and 11.3 observed at $m_{H}=125 \mathrm{GeV}$, the most stringent limit involving $H \rightarrow \tau \tau$ at the Tevatron. The $\ell \tau j j$ combination contributes to the 
Table 7.2. Summary of all the major improvements completed for this analysis and their approximate effect on the overall limit.

\begin{tabular}{cc}
\hline \hline Description & Approximate Improvement \\
\hline Certified new NN $\mathrm{N}_{\tau}$ & $5 \%$ \\
Used MVA Electron ID & $10 \%$ \\
Implemented EJets_OR Trigger & $5 \%$ \\
Optimized $Z \rightarrow$ ee Selections & $5 \%$ \\
Utilized MMC for di-tau Mass Calculations & $2 \%$ \\
Implemented 15 New BDT Variables in MVA & $15 \%$ \\
Created T + W Subsample Approach & $10 \%$ \\
Created Global BDT & Smooths limits \\
\hline \hline
\end{tabular}

complete Higgs picture as the discovery unfolds at the Tevatron and LHC. Finally, the Global BDT technique developed for the first time in this analysis can be implemented in any new physics search using multivariate trainings as a function of a particular parameter. 
Table 7.3. Comparison of limits with the previous analysis using $4.3 \mathrm{fb}^{-1}$ and the percent improvement of the expected limit. The doubling of luminosity only accounts for $\sim 30 \%$ of the improvement.

\begin{tabular}{c|cc|cc|c}
\hline \hline & \multicolumn{2}{|c|}{$4.3 \mathrm{fb}^{-1}$} & \multicolumn{2}{|c|}{$9.7 \mathrm{fb}^{-1}$} & Impr. \\
$m_{H}$ & exp. & obs. & exp. & obs. & exp. \\
\hline 105 & 29.4 & 29.6 & 14.9 & 11.8 & $49.3 \%$ \\
110 & 32.2 & 32.2 & 14.4 & 12.5 & $55.3 \%$ \\
115 & 34.0 & 34.0 & 14.2 & 11.7 & $58.2 \%$ \\
120 & 35.4 & 35.4 & 14.5 & 15.3 & $59.0 \%$ \\
125 & 53.2 & 53.2 & 14.6 & 16.0 & $72.6 \%$ \\
130 & 41.1 & 41.1 & 18.3 & 19.5 & $55.5 \%$ \\
135 & 44.2 & 44.2 & 20.4 & 21.4 & $53.6 \%$ \\
140 & 55.8 & 55.8 & 24.4 & 26.3 & $56.3 \%$ \\
145 & 46.7 & 46.7 & 29.8 & 36.0 & $36.2 \%$ \\
150 & 50.8 & 50.8 & 24.8 & 30.8 & $51.2 \%$ \\
\hline \hline
\end{tabular}

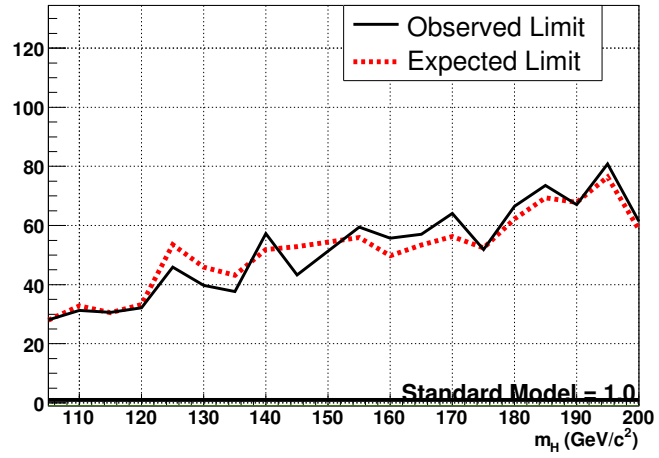

(a)

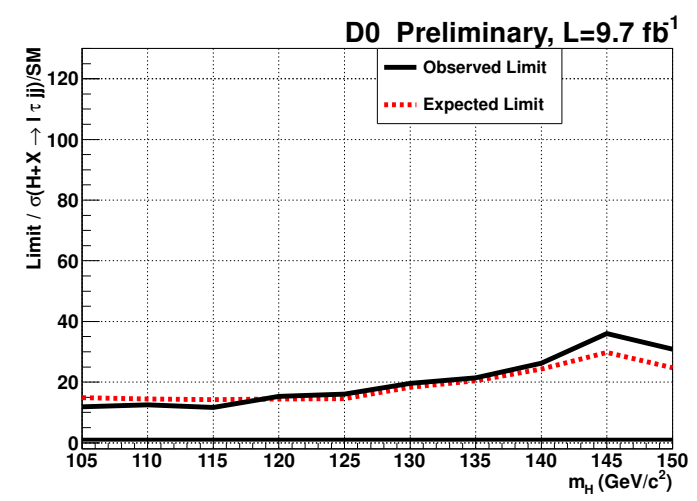

(b)

Figure 7.10. Comparison of (a) the previous and (b) the new e $j j j$ limits. The Global BDT method achieves the smoothly varying limit and all the various improvements combined to give more that $50 \%$ improvement in expected limits. Note the different $\mathrm{x}$ axis scales. 


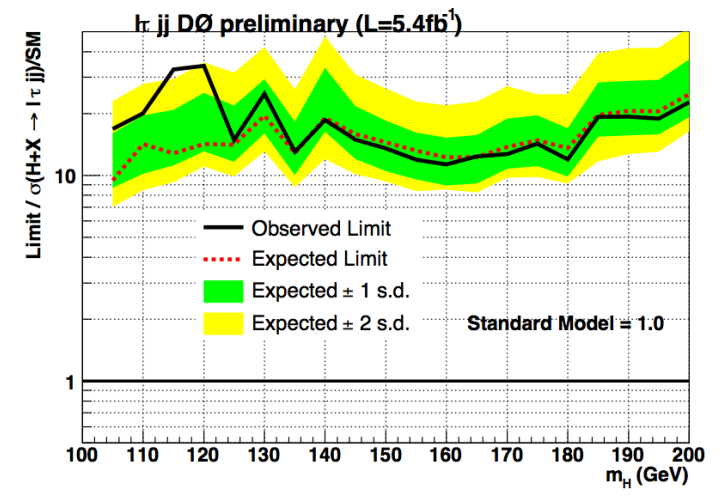

(a)

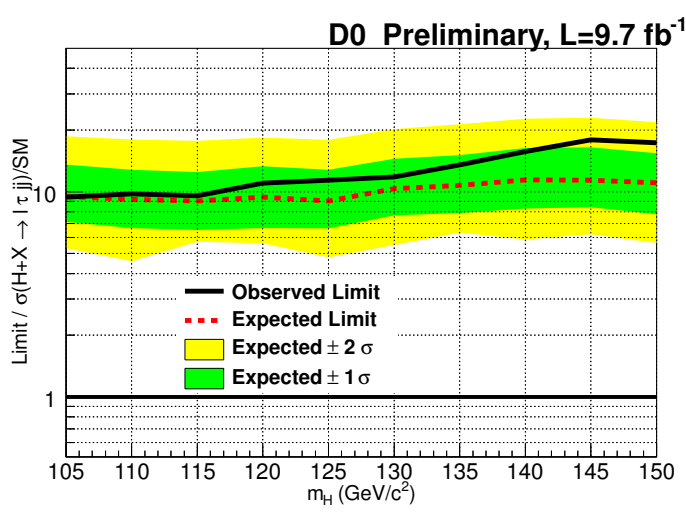

(b)

Figure 7.11. Comparison of (a) the previous and (b) the new $\ell \tau j j$ limits. There is an overall $\sim 20 \%$ improvement, as well as a much more smoothly varying distribution. Note the different $\mathrm{x}$-axis scales. 
APPENDIX A

EMinclusive Dataset 
This appendix lists the EMinclusive data samples on which the object selection is applied. These datasets amount to over 100 TB and are generated by the Common Samples Group. They are further skimmed by the analyzers to include at least one tau, a process that reduced the total file size to only a few TB.

- CSG_CAF_EMinclusive_PASS3_p17.09.03

- CSG_CAF_EMinclusive_PASS3_p17.09.06

- CSG_CAF_EMinclusive_PASS3_p17.09.06b

- CSG_CAF_EMinclusive_PASS4_p21.08.00

- CSG_CAF_EMinclusive_PASS4_p21.10.00_p20.12.01

- CSG_CAF_EMinclusive_PASS4_p21.10.00_p20.12.02

- CSG_CAF_EMinclusive_PASS4_p21.10.00_p20.12.04

- CSG_CAF_EMinclusive_PASS4_p21.12.00_p20.12.05_allfix

- CSG_CAF_EMinclusive_PASS5_p21.18.00_p20.16.07_fix

- CSG_CAF_EMinclusive_PASS5_p21.18.00_p20.16.07_summer2010

- CSG_CAF_EMinclusive_PASS6_p21.20.00_p20.18.02b

- CSG_CAF_EMinclusive_PASS6_p21.20.00_p20.18.02b_fix

- CSG_CAF_EMinclusive_PASS6_p21.21.00_p20.18.03

- CSG_CAF_EMinclusive_PASS6_p21.22.00_p20.18.04

- CSG_CAF_EMinclusive_PASS6_p21.22.00_p20.18.05 
APPENDIX B

MC Generation Tables 
This appendix contains Tables B.1, B.2, B.3, and B.4 which list the Monte Carlo signal processes, next-to-leading order cross sections, request IDs, and the number of events. 
Table B.1. The PYTHIA MC samples for signal processes, their cross-sections, MC request ID's, and the number of events generated.

\begin{tabular}{|c|c|c|c|}
\hline Sample & $\sigma_{\mathrm{NLO}}(\mathrm{pb})$ & MC req-id & $N_{\text {events }}$ \\
\hline$H Z \rightarrow b b \tau \tau, m_{H}=105 \mathrm{GeV}$ & $3.81 \times 10^{-3}$ & 97877 & $100 \mathrm{~K}$ \\
\hline$H Z \rightarrow b b \tau \tau, m_{H}=110 \mathrm{GeV}$ & $3.17 \times 10^{-3}$ & 94732 & $107 \mathrm{~K}$ \\
\hline$H Z \rightarrow b b \tau \tau, m_{H}=115 \mathrm{GeV}$ & $2.64 \times 10^{-3}$ & 97878 & $100 \mathrm{~K}$ \\
\hline$H Z \rightarrow b \bar{b} \tau \tau, m_{H}=120 \mathrm{GeV}$ & $2.10 \times 10^{-3}$ & 94733 & $104 \mathrm{~K}$ \\
\hline$H Z \rightarrow b b \tau \tau, m_{H}=125 \mathrm{GeV}$ & $1.64 \times 10^{-3}$ & 97879 & $100 \mathrm{~K}$ \\
\hline$H Z \rightarrow b \bar{b} \tau \tau, m_{H}=130 \mathrm{GeV}$ & $1.24 \times 10^{-3}$ & 97880 & $100 \mathrm{~K}$ \\
\hline$H Z \rightarrow b b \tau \tau, m_{H}=135 \mathrm{GeV}$ & $0.90 \times 10^{-3}$ & 97881 & $100 \mathrm{~K}$ \\
\hline$H Z \rightarrow b \bar{b} \tau \tau, m_{H}=140 \mathrm{GeV}$ & $0.61 \times 10^{-3}$ & 94734 & $100 \mathrm{~K}$ \\
\hline$H Z \rightarrow b \bar{b} \tau \tau, m_{H}=145 \mathrm{GeV}$ & $0.40 \times 10^{-3}$ & 97822 & $100 \mathrm{~K}$ \\
\hline$H Z \rightarrow b \bar{b} \tau \tau, m_{H}=150 \mathrm{GeV}$ & $0.24 \times 10^{-3}$ & 94735 & $102 \mathrm{~K}$ \\
\hline$Z H \rightarrow q \bar{q} \tau \tau, m_{H}=105 \mathrm{GeV}$ & $7.87 \times 10^{-3}$ & 102175,89797 & $104 \mathrm{~K}$ \\
\hline$Z H \rightarrow q \bar{q} \tau \tau, m_{H}=110 \mathrm{GeV}$ & $6.62 \times 10^{-3}$ & 94612 & $103 \mathrm{~K}$ \\
\hline$Z H \rightarrow q \bar{q} \tau \tau, m_{H}=115 \mathrm{GeV}$ & $5.55 \times 10^{-3}$ & 102176,89798 & $102 \mathrm{~K}$ \\
\hline$Z H \rightarrow q \bar{q} \tau \tau, m_{H}=120 \mathrm{GeV}$ & $4.46 \times 10^{-3}$ & 94613 & $107 \mathrm{~K}$ \\
\hline$Z H \rightarrow q \bar{q} \tau \tau, m_{H}=125 \mathrm{GeV}$ & $3.51 \times 10^{-3}$ & 102177,89799 & $103 \mathrm{~K}$ \\
\hline$Z H \rightarrow q \bar{q} \tau \tau, m_{H}=130 \mathrm{GeV}$ & $2.67 \times 10^{-3}$ & 94614 & $100 \mathrm{~K}$ \\
\hline$Z H \rightarrow q \bar{q} \tau \tau, m_{H}=135 \mathrm{GeV}$ & $1.94 \times 10^{-3}$ & 102178,89800 & $102 \mathrm{~K}$ \\
\hline$Z H \rightarrow q \bar{q} \tau \tau, m_{H}=140 \mathrm{GeV}$ & $1.34 \times 10^{-3}$ & 94615 & $100 \mathrm{~K}$ \\
\hline$Z H \rightarrow q \bar{q} \tau \tau, m_{H}=145 \mathrm{GeV}$ & $0.89 \times 10^{-3}$ & 102179,89801 & $102 \mathrm{~K}$ \\
\hline$Z H \rightarrow q \bar{q} \tau \tau, m_{H}=150 \mathrm{GeV}$ & $0.53 \times 10^{-3}$ & 94616 & $103 \mathrm{~K}$ \\
\hline$W H \rightarrow q \bar{q}^{\prime} \tau \tau, m_{H}=105 \mathrm{GeV}$ & $12.92 \times 10^{-3}$ & 89892,97857 & $153 \mathrm{~K}$ \\
\hline$W H \rightarrow q \bar{q}^{\prime} \tau \tau, m_{H}=110 \mathrm{GeV}$ & $10.77 \times 10^{-3}$ & 94592 & $102 \mathrm{~K}$ \\
\hline$W H \rightarrow q \bar{q}^{\prime} \tau \tau, m_{H}=115 \mathrm{GeV}$ & $8.97 \times 10^{-3}$ & 89893,97858 & $102 \mathrm{~K}$ \\
\hline$W H \rightarrow q \bar{q}^{\prime} \tau \tau, m_{H}=120 \mathrm{GeV}$ & $7.16 \times 10^{-3}$ & 94593 & $100 \mathrm{~K}$ \\
\hline$W H \rightarrow q \bar{q}^{\prime} \tau \tau, m_{H}=125 \mathrm{GeV}$ & $5.59 \times 10^{-3}$ & 89894,97859 & $104 \mathrm{~K}$ \\
\hline$W H \rightarrow q \bar{q}^{\prime} \tau \tau, m_{H}=130 \mathrm{GeV}$ & $4.20 \times 10^{-3}$ & 94594 & $100 \mathrm{~K}$ \\
\hline$W H \rightarrow q \bar{q}^{\prime} \tau \tau, m_{H}=135 \mathrm{GeV}$ & $3.03 \times 10^{-3}$ & 89895,97860 & $104 \mathrm{~K}$ \\
\hline$W H \rightarrow q \bar{q}^{\prime} \tau \tau, m_{H}=140 \mathrm{GeV}$ & $2.07 \times 10^{-3}$ & 94595 & $100 \mathrm{~K}$ \\
\hline$W H \rightarrow q \bar{q}^{\prime} \tau \tau, m_{H}=145 \mathrm{GeV}$ & $1.34 \times 10^{-3}$ & 89896,97861 & $104 \mathrm{~K}$ \\
\hline$W H \rightarrow q \bar{q}^{\prime} \tau \tau, m_{H}=150 \mathrm{GeV}$ & $0.81 \times 10^{-3}$ & 94596 & $102 \mathrm{~K}$ \\
\hline$q q^{\prime} \rightarrow q q^{\prime} H(\rightarrow \tau \tau), m_{H}=105 \mathrm{GeV}$ & $7.30 \times 10^{-3}$ & $94238,97086,110873$ & $302 \mathrm{~K}$ \\
\hline$q q^{\prime} \rightarrow q q^{\prime} H(\rightarrow \tau \tau), m_{H}=110 \mathrm{GeV}$ & $6.64 \times 10^{-3}$ & 97093, 110874 & $260 \mathrm{~K}$ \\
\hline$q q^{\prime} \rightarrow q q^{\prime} H(\rightarrow \tau \tau), m_{H}=115 \mathrm{GeV}$ & $5.76 \times 10^{-3}$ & $94239,97087,110875$ & $302 \mathrm{~K}$ \\
\hline$q q^{\prime} \rightarrow q q^{\prime} H(\rightarrow \tau \tau), m_{H}=120 \mathrm{GeV}$ & $4.86 \times 10^{-3}$ & 97094,110876 & $250 \mathrm{~K}$ \\
\hline$q q^{\prime} \rightarrow q q^{\prime} H(\rightarrow \tau \tau), m_{H}=125 \mathrm{GeV}$ & $4.12 \times 10^{-3}$ & $94240,97085,110877$ & $382 \mathrm{~K}$ \\
\hline$q q^{\prime} \rightarrow q q^{\prime} H(\rightarrow \tau \tau), m_{H}=130 \mathrm{GeV}$ & $3.31 \times 10^{-3}$ & 97095,110878 & $253 \mathrm{~K}$ \\
\hline$q q^{\prime} \rightarrow q q^{\prime} H(\rightarrow \tau \tau), m_{H}=135 \mathrm{GeV}$ & $2.54 \times 10^{-3}$ & $94241,97088,110879$ & $305 \mathrm{~K}$ \\
\hline$q q^{\prime} \rightarrow q q^{\prime} H(\rightarrow \tau \tau), m_{H}=140 \mathrm{GeV}$ & $1.833 \times 10^{-3}$ & 97096,110880 & $252 \mathrm{~K}$ \\
\hline$q q^{\prime} \rightarrow q q^{\prime} H(\rightarrow \tau \tau), m_{H}=145 \mathrm{GeV}$ & $1.27 \times 10^{-3}$ & $94242,97089,110881$ & $309 \mathrm{~K}$ \\
\hline$q q^{\prime} \rightarrow q q^{\prime} H(\rightarrow \tau \tau), m_{H}=150 \mathrm{GeV}$ & $0.81 \times 10^{-3}$ & 97097,110882 & $251 \mathrm{~K}$ \\
\hline
\end{tabular}


Table B.2. The PYTHIA MC samples for signal processes, their cross-sections, MC request ID's, and the number of events generated.

\begin{tabular}{|c|c|c|c|}
\hline Sample & $\sigma_{\mathrm{NLO}}(\mathrm{pb})$ & MC req-id & $N_{\text {events }}$ \\
\hline$W H \rightarrow H(\rightarrow W W)$ incl., $m_{H}=105 \mathrm{GeV}$ & $5.61 \times 10^{-3}$ & 110725 & $203 \mathrm{~K}$ \\
\hline$W H \rightarrow H(\rightarrow W W)$ incl., $m_{H}=110 \mathrm{GeV}$ & $9.54 \times 10^{-3}$ & 110723 & $202 \mathrm{~K}$ \\
\hline$W H \rightarrow H(\rightarrow W W)$ incl., $m_{H}=115 \mathrm{GeV}$ & $14.79 \times 10^{-3}$ & 110726 & $203 \mathrm{~K}$ \\
\hline$W H \rightarrow H(\rightarrow W W)$ incl., $m_{H}=120 \mathrm{GeV}$ & $21.06 \times 10^{-3}$ & 103313 & $200 \mathrm{~K}$ \\
\hline$W H \rightarrow H(\rightarrow W W)$ incl., $m_{H}=125 \mathrm{GeV}$ & $27.78 \times 10^{-3}$ & 110727 & $203 \mathrm{~K}$ \\
\hline$W H \rightarrow H(\rightarrow W W)$ incl., $m_{H}=130 \mathrm{GeV}$ & $34.22 \times 10^{-3}$ & 110724 & $202 \mathrm{~K}$ \\
\hline$W H \rightarrow H(\rightarrow W W)$ incl., $m_{H}=135 \mathrm{GeV}$ & $39.63 \times 10^{-3}$ & 110728 & $202 \mathrm{~K}$ \\
\hline$W H \rightarrow H(\rightarrow W W)$ incl., $m_{H}=140 \mathrm{GeV}$ & $43.61 \times 10^{-3}$ & 103314 & $200 \mathrm{~K}$ \\
\hline$W H \rightarrow H(\rightarrow W W)$ incl., $m_{H}=145 \mathrm{GeV}$ & $45.95 \times 10^{-3}$ & 110729 & $203 \mathrm{~K}$ \\
\hline$W H \rightarrow H(\rightarrow W W)$ incl., $m_{H}=150 \mathrm{GeV}$ & $46.97 \times 10^{-3}$ & 103315 & $200 \mathrm{~K}$ \\
\hline$q q^{\prime} \rightarrow q q^{\prime} H(\rightarrow W W), m_{H}=105 \mathrm{GeV}$ & $0.226 \times 10^{-3}$ & 91832,92335 & $206 \mathrm{~K}$ \\
\hline$q q^{\prime} \rightarrow q q^{\prime} H(\rightarrow W W), m_{H}=110 \mathrm{GeV}$ & $0.419 \times 10^{-3}$ & 89832,92336 & $204 \mathrm{~K}$ \\
\hline$q q^{\prime} \rightarrow q q^{\prime} H(\rightarrow W W), m_{H}=115 \mathrm{GeV}$ & $0.686 \times 10^{-3}$ & 89833,92337 & $205 \mathrm{~K}$ \\
\hline$q q^{\prime} \rightarrow q q^{\prime} H(\rightarrow W W), m_{H}=120 \mathrm{GeV}$ & $1.026 \times 10^{-3}$ & 89834,92338 & $205 \mathrm{~K}$ \\
\hline$q q^{\prime} \rightarrow q q^{\prime} H(\rightarrow W W), m_{H}=125 \mathrm{GeV}$ & $1.470 \times 10^{-3}$ & 89835,92339 & $208 \mathrm{~K}$ \\
\hline$q q^{\prime} \rightarrow q q^{\prime} H(\rightarrow W W), m_{H}=130 \mathrm{GeV}$ & $1.931 \times 10^{-3}$ & 89836,92340 & $204 \mathrm{~K}$ \\
\hline$q q^{\prime} \rightarrow q q^{\prime} H(\rightarrow W W), m_{H}=135 \mathrm{GeV}$ & $2.366 \times 10^{-3}$ & 89837,92341 & $212 \mathrm{~K}$ \\
\hline$q q^{\prime} \rightarrow q q^{\prime} H(\rightarrow W W), m_{H}=140 \mathrm{GeV}$ & $2.714 \times 10^{-3}$ & 89838,92342 & $206 \mathrm{~K}$ \\
\hline$q q^{\prime} \rightarrow q q^{\prime} H(\rightarrow W W), m_{H}=145 \mathrm{GeV}$ & $3.052 \times 10^{-3}$ & 89839,92343 & $207 \mathrm{~K}$ \\
\hline$q q^{\prime} \rightarrow q q^{\prime} H(\rightarrow W W), m_{H}=150 \mathrm{GeV}$ & $3.304 \times 10^{-3}$ & 89840,92344 & $201 \mathrm{~K}$ \\
\hline
\end{tabular}

Table B.3. The PYTHIA MC samples for signal processes, their cross-sections, MC request ID's, and the number of events generated.

\begin{tabular}{|c|c|c|c|}
\hline Sample & $\sigma_{\mathrm{NLO}}(\mathrm{pb})$ & MC req-id & $N_{\text {events }}$ \\
\hline$Z H \rightarrow H(\rightarrow W W)$ incl., $m_{H}=105 \mathrm{GeV}$ & $3.32 \times 10^{-3}$ & 113553 & $200.5 \mathrm{~K}$ \\
\hline$Z H \rightarrow H(\rightarrow W W)$ incl., $m_{H}=110 \mathrm{GeV}$ & $5.699 \times 10^{-3}$ & 113554 & $203.5 \mathrm{~K}$ \\
\hline$Z H \rightarrow H(\rightarrow W W)$ incl., $m_{H}=115 \mathrm{GeV}$ & $9.012 \times 10^{-3}$ & 113555 & $200.75 \mathrm{~K}$ \\
\hline$Z H \rightarrow H(\rightarrow W W)$ incl., $m_{H}=120 \mathrm{GeV}$ & $12.822 \times 10^{-3}$ & 113556 & $203.75 \mathrm{~K}$ \\
\hline$Z H \rightarrow H(\rightarrow W W)$ incl., $m_{H}=125 \mathrm{GeV}$ & $17.04 \times 10^{-3}$ & 113557 & $202.5 \mathrm{~K}$ \\
\hline$Z H \rightarrow H(\rightarrow W W)$ incl., $m_{H}=130 \mathrm{GeV}$ & $21.19 \times 10^{-3}$ & 113558 & $203 \mathrm{~K}$ \\
\hline$Z H \rightarrow H(\rightarrow W W)$ incl., $m_{H}=135 \mathrm{GeV}$ & $24.633 \times 10^{-3}$ & 113559 & $203.5 \mathrm{~K}$ \\
\hline$Z H \rightarrow H(\rightarrow W W)$ incl., $m_{H}=140 \mathrm{GeV}$ & $27.038 \times 10^{-3}$ & 113560 & $201.2 \mathrm{~K}$ \\
\hline$Z H \rightarrow H(\rightarrow W W)$ incl., $m_{H}=145 \mathrm{GeV}$ & $28.98 \times 10^{-3}$ & 111252 & $200 \mathrm{~K}$ \\
\hline$Z H \rightarrow H(\rightarrow W W)$ incl., $m_{H}=150 \mathrm{GeV}$ & $29.631 \times 10^{-3}$ & 113561 & $200 \mathrm{~K}$ \\
\hline
\end{tabular}


Table B.4. The PYTHIA MC samples for signal processes, their cross-sections, MC request ID's, and the number of events generated. The abbreviation "lept." means that the $W$ bosons are decayed to any lepton $(e, \mu$, or $\tau)$.

\begin{tabular}{|c|c|c|c|}
\hline Sample & $\sigma_{\mathrm{NNLO}}(\mathrm{pb})$ & MC req-id & $N_{\text {events }}$ \\
\hline$g g \rightarrow H(\rightarrow \tau \tau)$ incl., $m_{H}=105 \mathrm{GeV}$ & $126.5 \times 10^{-3}$ & 94232 & $103 \mathrm{~K}$ \\
\hline$g g \rightarrow H(\rightarrow \tau \tau)$ incl., $m_{H}=110 \mathrm{GeV}$ & $107.1 \times 10^{-3}$ & 88854 & $530 \mathrm{~K}$ \\
\hline$g g \rightarrow H(\rightarrow \tau \tau)$ incl., $m_{H}=115 \mathrm{GeV}$ & $90.4 \times 10^{-3}$ & 94233 & $105 \mathrm{~K}$ \\
\hline$g g \rightarrow H(\rightarrow \tau \tau)$ incl., $m_{H}=120 \mathrm{GeV}$ & $74.2 \times 10^{-3}$ & 88855 & $517 \mathrm{~K}$ \\
\hline$g g \rightarrow H(\rightarrow \tau \tau)$ incl., $m_{H}=125 \mathrm{GeV}$ & $59.2 \times 10^{-3}$ & 94234 & $103 \mathrm{~K}$ \\
\hline$g g \rightarrow H(\rightarrow \tau \tau)$ incl., $m_{H}=130 \mathrm{GeV}$ & $45.5 \times 10^{-3}$ & 88856 & $510 \mathrm{~K}$ \\
\hline$g g \rightarrow H(\rightarrow \tau \tau)$ incl., $m_{H}=135 \mathrm{GeV}$ & $33.6 \times 10^{-3}$ & 94235 & $101 \mathrm{~K}$ \\
\hline$g g \rightarrow H(\rightarrow \tau \tau)$ incl., $m_{H}=140 \mathrm{GeV}$ & $23.7 \times 10^{-3}$ & 88857 & $506 \mathrm{~K}$ \\
\hline$g g \rightarrow H(\rightarrow \tau \tau)$ incl., $m_{H}=145 \mathrm{GeV}$ & $15.79 \times 10^{-3}$ & 94236 & $100 \mathrm{~K}$ \\
\hline$g g \rightarrow H(\rightarrow \tau \tau)$ incl., $m_{H}=150 \mathrm{GeV}$ & $9.74 \times 10^{-3}$ & 88858 & $535 \mathrm{~K}$ \\
\hline$g g \rightarrow H(\rightarrow W W)$ lept., $m_{H}=105 \mathrm{GeV}$ & $14.73 \times 10^{-3}$ & $92314,93494,93495$ & $720 \mathrm{~K}$ \\
\hline$g g \rightarrow H(\rightarrow W W)$ lept., $m_{H}=110 \mathrm{GeV}$ & $12.65 \times 10^{-3}$ & $90383,92315,93496,93497$ & $700 \mathrm{~K}$ \\
\hline$g g \rightarrow H(\rightarrow W W)$ lept., $m_{H}=115 \mathrm{GeV}$ & $10.76 \times 10^{-3}$ & $90384,92316,93498,93499$ & $70 \mathrm{~K}$ \\
\hline$g g \rightarrow H(\rightarrow W W)$ lept., $m_{H}=120 \mathrm{GeV}$ & $15.65 \times 10^{-3}$ & $93500,93501,92317$ & $608 \mathrm{~K}$ \\
\hline$g g \rightarrow H(\rightarrow W W)$ lept., $m_{H}=125 \mathrm{GeV}$ & $21.09 \times 10^{-3}$ & $90385,92318,93502,93503$ & $920 \mathrm{~K}$ \\
\hline$g g \rightarrow H(\rightarrow W W)$ lept., $m_{H}=130 \mathrm{GeV}$ & $26.51 \times 10^{-3}$ & $89357,92319,93504,93505$ & $701 \mathrm{~K}$ \\
\hline$g g \rightarrow H(\rightarrow W W)$ lept., $m_{H}=135 \mathrm{GeV}$ & $31.36 \times 10^{-3}$ & $90386,92320,93506,93507$ & $708 \mathrm{~K}$ \\
\hline$g g \rightarrow H(\rightarrow W W)$ lept., $m_{H}=140 \mathrm{GeV}$ & $35.19 \times 10^{-3}$ & $92321,93508,93509$ & $604 \mathrm{~K}$ \\
\hline$g g \rightarrow H(\rightarrow W W)$ lept., $m_{H}=145 \mathrm{GeV}$ & $37.94 \times 10^{-3}$ & $90387,92322,93510,93511$ & $709 \mathrm{~K}$ \\
\hline$g g \rightarrow H(\rightarrow W W)$ lept., $m_{H}=150 \mathrm{GeV}$ & $39.64 \times 10^{-3}$ & $89353,92323,93512,93513$ & $712 \mathrm{~K}$ \\
\hline
\end{tabular}




\section{APPENDIX C}

Jet Shape Systematic Modeling 
In this appendix are the jet related systematics (Jet ID, Jet Energy Resolution, Jet Energy Scale and vertex confirmation) with coarsened bins to alleviate statistical fluctuations. The $-1 \sigma$ changes to the jet corrections are made and propagated through the final BDTs to obtain the modified shapes. The $-1 \sigma$ shape changes are then compared to the original BDT distributions by

$$
\delta \mathrm{BDT}_{-1 \sigma}=\frac{\mathrm{BDT}_{\text {nominal }}-\mathrm{BDT}_{-1 \sigma}}{\mathrm{BDT}_{\text {nominal }}}
$$

$\mathrm{BDT}_{-1 \sigma}$ is then inverted to give the symmetric $\mathrm{BDT}_{+1 \sigma}$ relative systematic uncertainties. These uncertainties are obtained for the $\tau \tau$ subsample and the $W W$ subsample at $m_{H}=115 \mathrm{GeV}$ and applied to the $\mathrm{T}$ and $\mathrm{W}$ subsamples for $m_{H} \leq 150$ $\mathrm{GeV}$. 
Signals 115 jid systematics

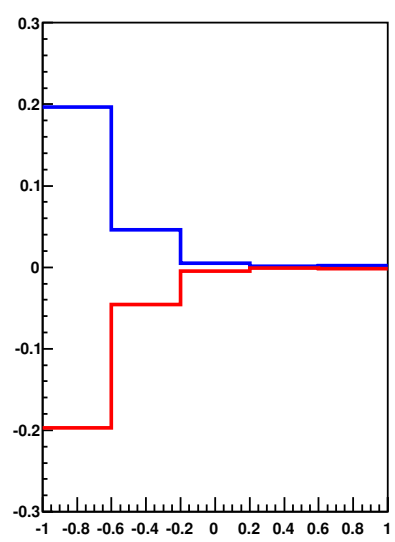

Signals 115 jid systematics

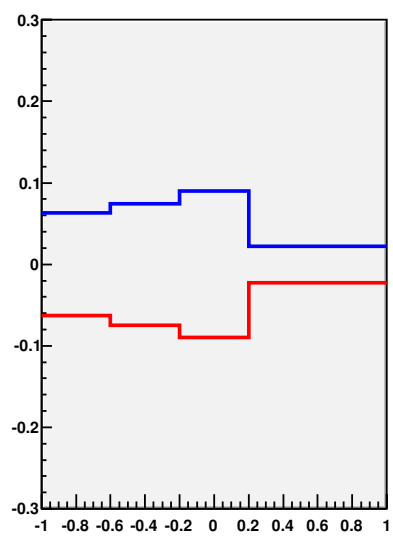

Zjets 115 jid systematics

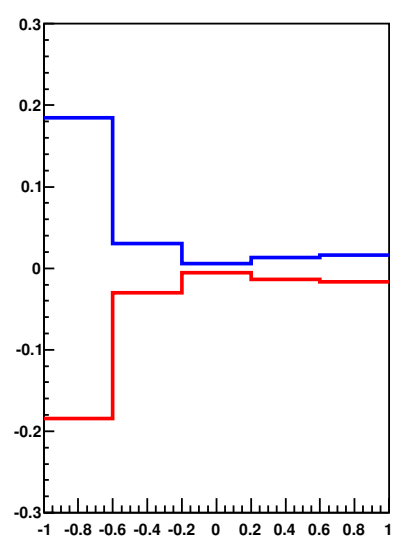

Zjets 115 jid systematics

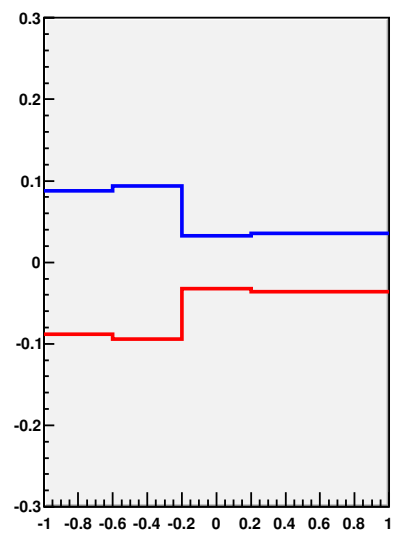

Wjets+tt 115 jid systematics

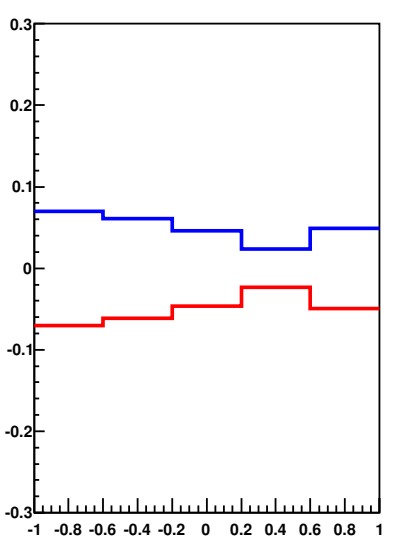

Wjets+tt 115 jid systematics

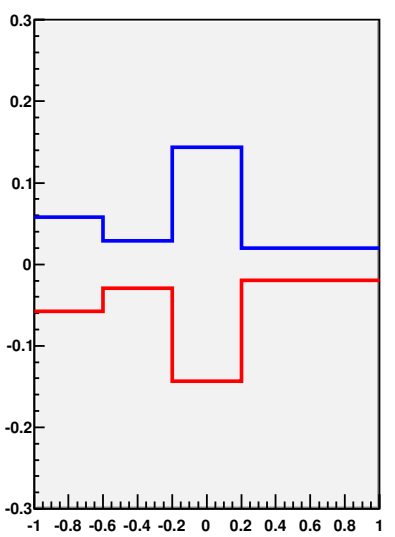

Figure C.1. Normalized $\delta \mathrm{BDT}_{ \pm 1 \sigma}$ outputs of the jet ID and reconstruction efficiencies, for (top row) the $\mathrm{T}$ subsample at $115 \mathrm{GeV}$ and for (bottom row) the $\mathrm{W}$ subsample at $115 \mathrm{GeV}$. The signals, $Z+$ jets and $W+$ jets $+t \bar{t}$ backgrounds are shown in the left, middle and right columns respectively. 

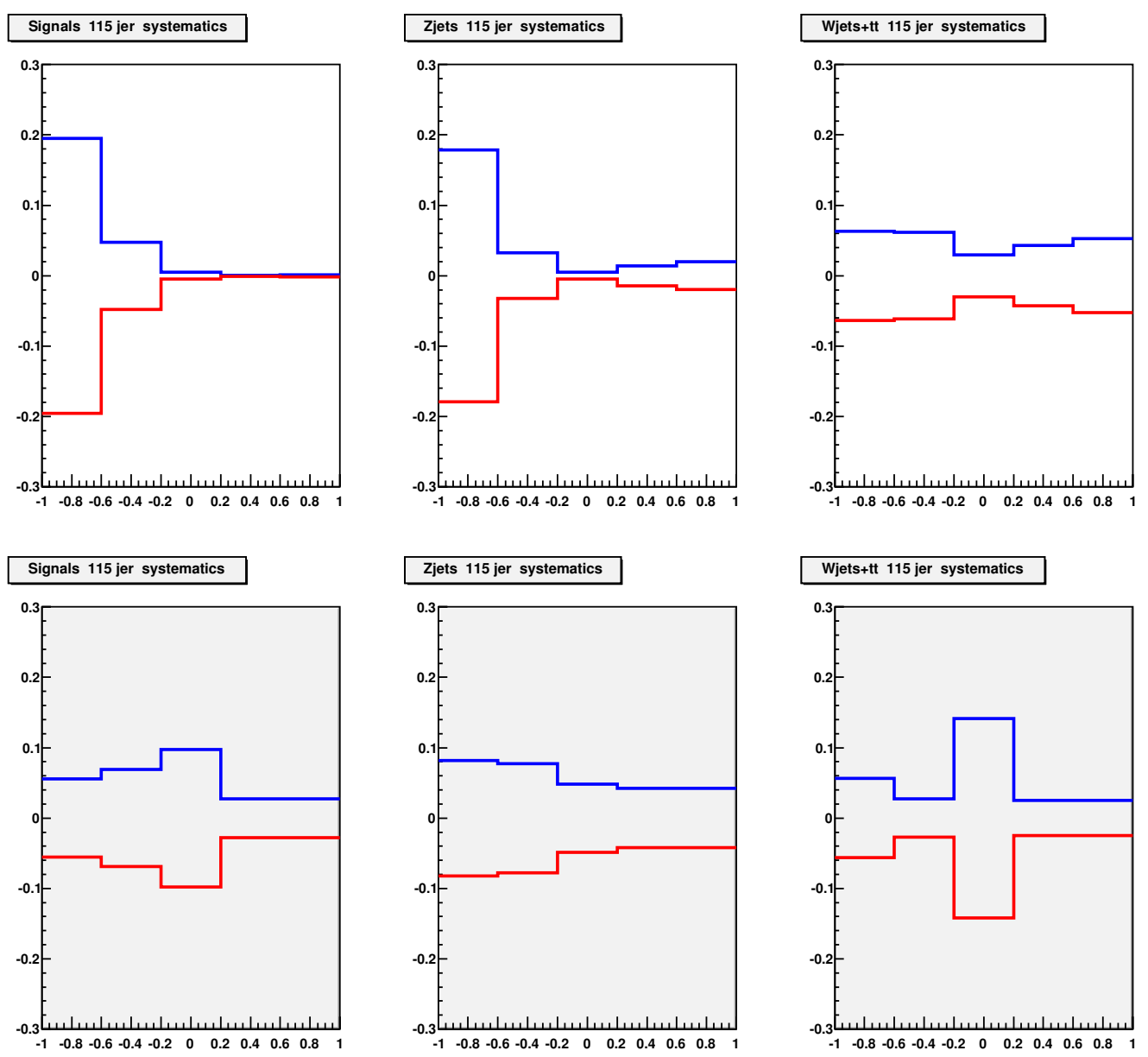

Figure C.2. Normalized $\delta \mathrm{BDT}_{ \pm 1 \sigma}$ outputs of the jet energy resolution, for (top row) the T subsample at $115 \mathrm{GeV}$ and for (bottom row) the $\mathrm{W}$ subsample at $115 \mathrm{GeV}$. The signals, $Z+$ jets and $W+$ jets $+t \bar{t}$ backgrounds are shown in the left, middle and right columns respectively. 

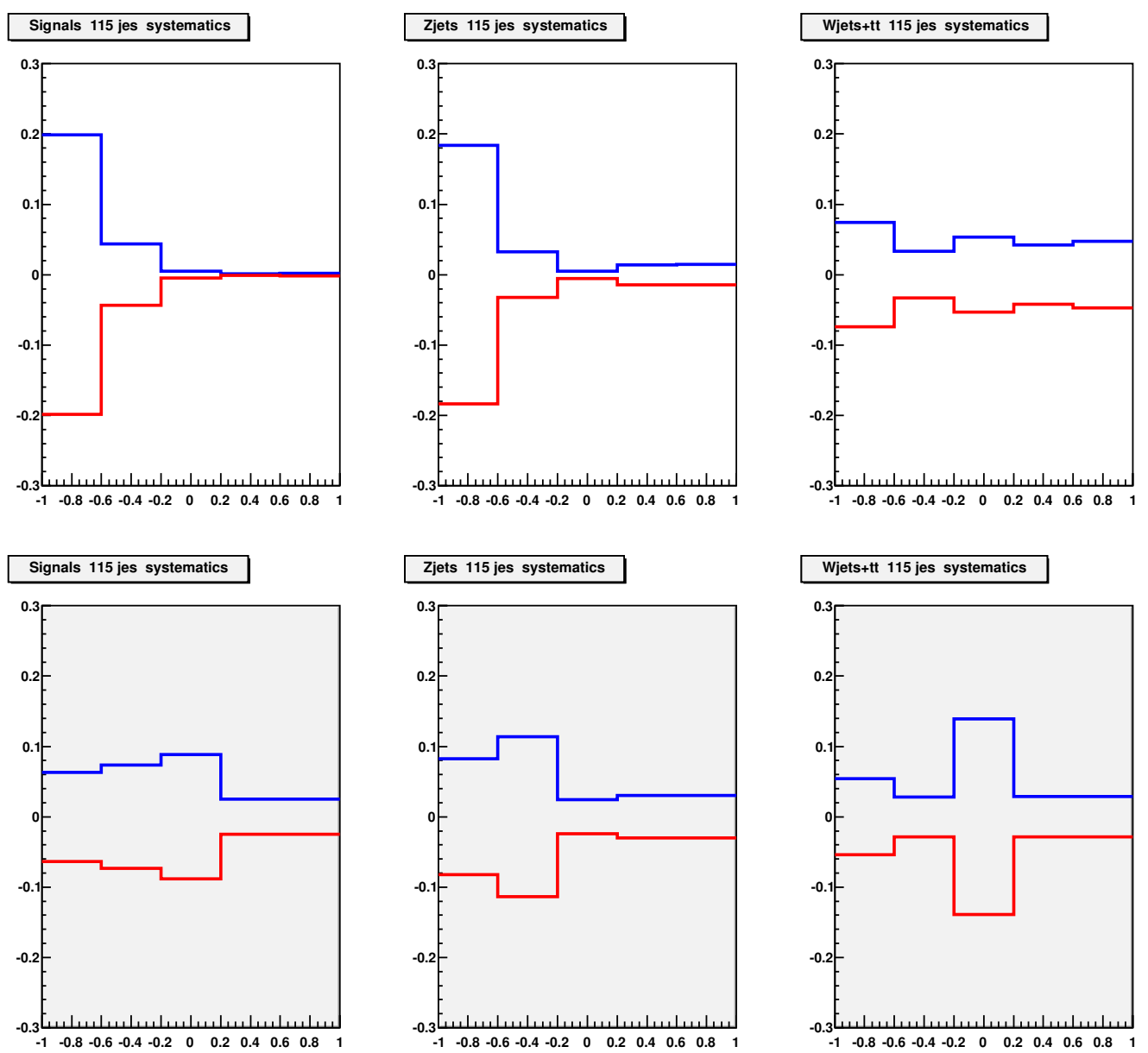

Figure C.3. Normalized $\delta \mathrm{BDT}_{ \pm 1 \sigma}$ outputs of the jet energy scale, for (top row) the $\mathrm{T}$ subsample at $115 \mathrm{GeV}$ and for (bottom row) the $\mathrm{W}$ subsample at $115 \mathrm{GeV}$. The signals, $Z+$ jets and $W+$ jets $+t \bar{t}$ backgrounds are shown in the left, middle and right columns respectively. 

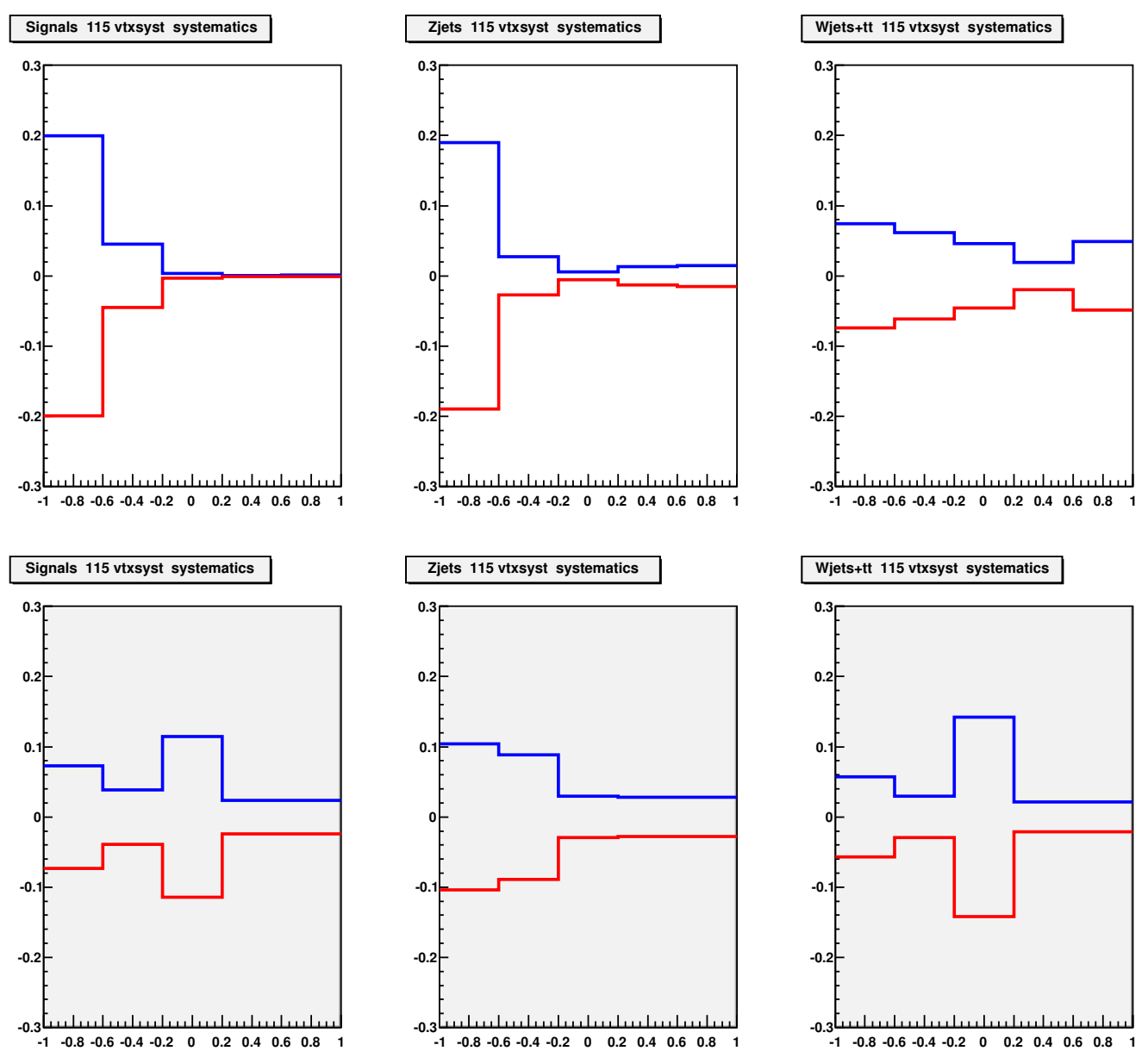

Figure C.4. Normalized $\delta \mathrm{BDT}_{ \pm 1 \sigma}$ outputs of the vertex confirmation, for (top row) the $\mathrm{T}$ subsample at $115 \mathrm{GeV}$ and for (bottom row) the $\mathrm{W}$ subsample at $115 \mathrm{GeV}$. The signals, $Z+$ jets and $W+$ jets $+t \bar{t}$ backgrounds are shown in the left, middle and right columns respectively. 
APPENDIX D

Epilogue 
On July 4, 2012 the LHC experiments ATLAS and CMS jointly announced the discovery of a new Higgs-like boson with mass around $126 \mathrm{GeV}$ using data from 2011 at $\sqrt{s}=7 \mathrm{TeV}$, and data from 2012 at $\sqrt{s}=8 \mathrm{TeV}$ totaling $\sim 10 \mathrm{fb}^{-1} \quad$ [34], [35]. The analyses from both experiments relied heavily on two channels: the low cross section $H \rightarrow \gamma \gamma$, and the very clean $H \rightarrow Z Z^{(*)} \rightarrow 4 \ell$. Figure D.1 (a) shows the ATLAS $4 \ell$ channel with roughly 7 excess events between $120<m_{4 \ell}<130 \mathrm{GeV}$. Figure D.1 (b) shows the CMS $\gamma \gamma$ channel with its small bump on the large decaying background. The significance of each individual channel was above $4 \sigma$, but when combining the channels within each experiment both ATLAS and CMS independently could claim discovery of a new Higgs-like boson with significance above $5 \sigma$. The excess can also be seen in Figure D.2 in the cross section limit plots.

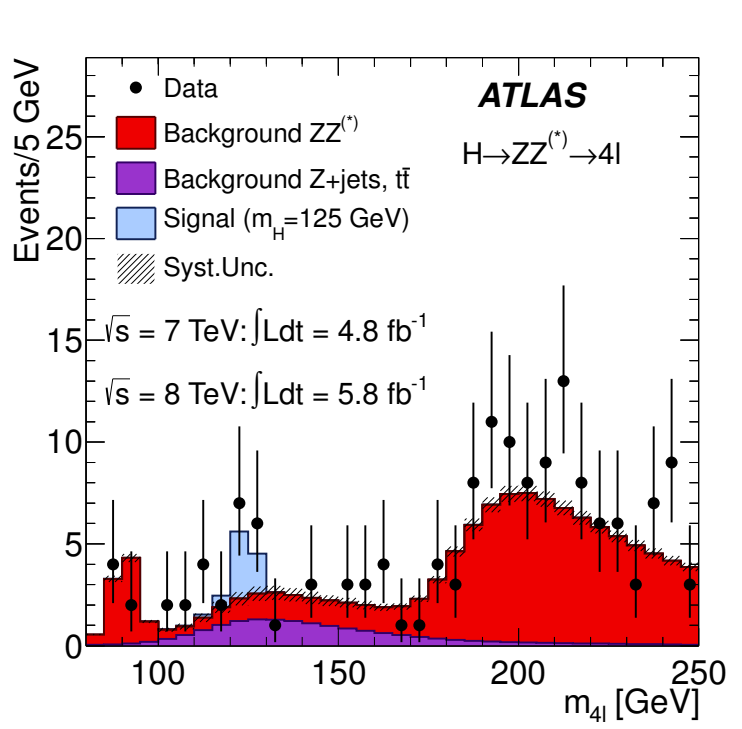

(a)

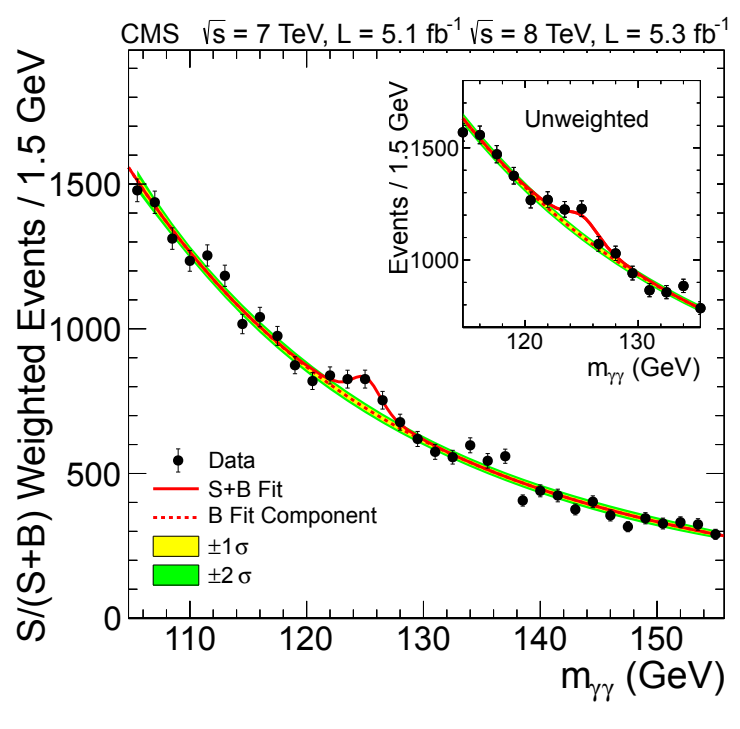

(b)

Figure D.1. (a) The dijet mass distribution used for discovery in the ATLAS $H \rightarrow$ $Z Z^{(*)} \rightarrow 4 \ell$ channel. (b) The invariant mass distribution used for discovery in the CMS $H \rightarrow \gamma \gamma$ channel. 


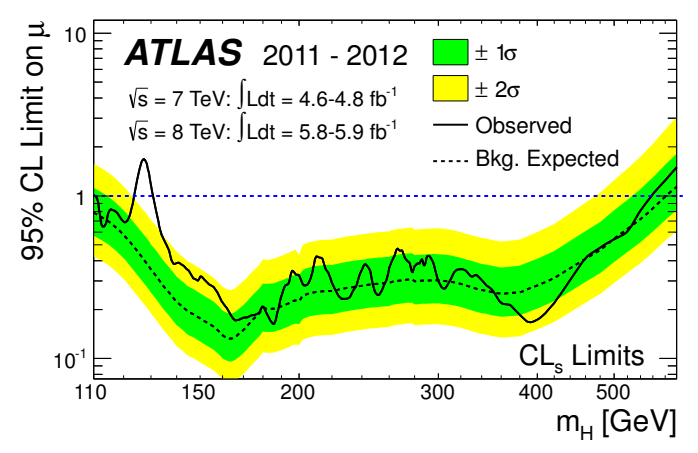

(a)

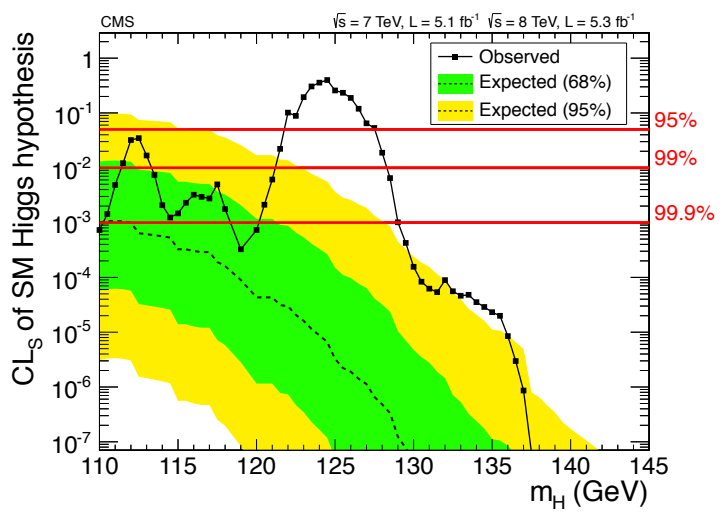

(b)

Figure D.2. The (a) ATLAS and (b) CMS limit plots showing an excess of data above $5 \sigma$ around $m_{H}=125 \mathrm{GeV}$. 


\section{Bibliography}

[1] S. L. Glashow, "Partial-symmetries of weak interactions," Nuclear Physics, vol. 22 , no. 4 , pp. $579-588,1961$.

[2] A. Salam, Elementary Particle Theory. Almqvist and Wiksell, Stockholm, 1968.

[3] M. L. Perl, G. S. Abrams, A. M. Boyarski, M. Breidenbach, D. D. Briggs, F. Bulos, W. Chinowsky, J. T. Dakin, G. J. Feldman, C. E. Friedberg, D. Fryberger, G. Goldhaber, G. Hanson, F. B. Heile, B. Jean-Marie, J. A. Kadyk, R. R. Larsen, A. M. Litke, D. Lüke, B. A. Lulu, V. Lüth, D. Lyon, C. C. Morehouse, J. M. Paterson, F. M. Pierre, T. P. Pun, P. A. Rapidis, B. Richter, B. Sadoulet, R. F. Schwitters, W. Tanenbaum, G. H. Trilling, F. Vannucci, J. S. Whitaker, F. C. Winkelmann, and J. E. Wiss, "Evidence for Anomalous Lepton Production in $e^{+}-e^{-}$Annihilation," Phys. Rev. Lett., vol. 35, pp. 1489-1492, Dec 1975.

[4] M. L. Perl, "Evidence for, and properties of, the new charged heavy lepton," 1977. Paper presented at the XII Rencontre de Moriond (Flaine, March 6-18, 1977; to be published in the Proceedings of the XII Recontre de Moriond, edited by Tran Thanh Van, R.M.I.E.M. Orsay.

[5] D. Griffiths, Introduction to Elementary Particle. John Wiley \& Sons Inc., 1987.

[6] Emmanuel A. Paschos, Electroweak Theory. Cambridge University Press, 2007.

[7] "The Goldstone Theorem for Real Dummies," Nov. 2007.

[8] P. W. Higgs, "Broken Symmetries and the Masses of Gauge Bosons," Phys. Rev. Lett., vol. 13, pp. 508-509, Oct 1964. 
[9] G. S. Guralnik, C. R. Hagen, and T. W. B. Kibble, "Global Conservation Laws and Massless Particles," Phys. Rev. Lett., vol. 13, pp. 585-587, Nov 1964.

[10] "Search for the Standard Model Higgs boson at LEP," Physics Letters B, vol. 565, no. 0 , pp. $61-75,2003$.

[11] J. Ellis, J. Espinosa, G. Giudice, A. Hoecker, and A. Riotto, "The probable fate of the Standard Model," Physics Letters B, vol. 679, no. 4, pp. 369 - 375, 2009.

[12] G. Aad et al. (ATLAS Collaboration), "Search for the Standard Model Higgs boson in the $H \rightarrow \tau \tau$ decay mode in $\sqrt{s}=7 \mathrm{TeV} p p$ collisions with ATLAS," 2012.

[13] S. Chatrchyan et al. (CMS Collaboration), "Search for neutral Higgs bosons decaying to tau pairs in $p p$ collisions at $\sqrt{s}=7 \mathrm{TeV}$," Physics Letters B, vol. 713, no. 2, pp. $68-90,2012$.

[14] Fermilab, "History of Fermi National Accelerator Laboratory," Dec. 2001.

[15] W. R. Innes, J. A. Appel, B. C. Brown, C. N. Brown, K. Ueno, T. Yamanouchi, S. W. Herb, D. C. Hom, L. M. Lederman, J. C. Sens, H. D. Snyder, J. K. Yoh, R. J. Fisk, A. S. Ito, H. Jöstlein, D. M. Kaplan, and R. D. Kephart, "Observation of Structure in the Upsilon Region," Phys. Rev. Lett., vol. 39, pp. 1240-1242, Nov 1977.

[16] F. Abe et al. (CDF Collaboration), "Observation of top quark production in $\bar{p} p$ collisions," Phys. Rev. Lett., vol. 74, pp. 2626-2631, 1995.

[17] S. Abachi et al. (DØ Collaboration), "Observation of the top quark," Phys. Rev. Lett., vol. 74, pp. 2632-2637, 1995. 
[18] K. Kodama et al. (DONUT Collaboration), "Observation of tau neutrino interactions," Physics Letters B, vol. 504, no. 3, pp. 218 - 224, 2001.

[19] Fermilab, "About Fermi National Accelerator Laboratory," 2002.

[20] V.M. Abazov et al. (DØ Collaboration), "The Upgraded DØ detector," Nuclear Instruments and Methods in Physics Research Section A: Accelerators, Spectrometers, Detectors and Associated Equipment, vol. 565, no. 2, pp. 463 - 537, 2006.

[21] P. Gutierrez (DØ Collaboration), "The DØ silicon tracker," Nuclear Instruments and Methods in Physics Research Section A: Accelerators, Spectrometers, Detectors and Associated Equipment, vol. 383, no. 1, pp. 21 - 26, 1996. Development and Application of Semiconductor Tracking Detectors.

[22] D. Lincoln (DØ Collaboration), "New technologies in the DØ central tracker upgrade," Nuclear Instruments and Methods in Physics Research Section A: Accelerators, Spectrometers, Detectors and Associated Equipment, vol. 379, no. 3, pp. $424-426$, 1996. Proceedings of the Sixth International Conference on Instrumentation for Experiments at e+ e- Colliders.

[23] J. Haley, B. Tuchming, and L. Zivkovic, "Dø MC Generators Group," Mar. 2011.

[24] S. Jadach, Z. Was, R. Decker, and J. H. Kuhn, "The tau decay library TAUOLA: Version 2.4," Comput.Phys.Commun., vol. 76, pp. 361-380, 1993.

[25] R. Madar and F. Couderc, "Tau lepton identification using MVA optimizations, CPS detector and bID tools," DØ Internal Notes, 2010. DØ-NOTE-6061.

[26] E. Busato and B. Andrieu, "Jet algorithms in the DØ Run II software: Description and users guide," DØ Internal Notes, 2004. DØ-NOTE-4457. 
[27] K. DeVaughan et al., "Jet Energy Scale Determination for DØ Run IIb," DØ Internal Notes, 2008. DØ-NOTE-5801.

[28] A. Schwartzman, "龙 Significance Algorithm in Run II Data," DØ Internal Notes, 2003. DØ-NOTE-4254.

[29] V.M. Abazov et al. (DØ Collaboration), "Search for the standard model Higgs boson in tau lepton final states," Physics Letters B, vol. 714, no. 25, pp. 237 $245,2012$.

[30] A. Elagin, P. Murat, A. Pranko, and A. Safonov, "A New Mass Reconstruction Technique for Resonances Decaying to di-tau," Nucl.Instrum.Meth., vol. A654, pp. 481-489, 2011.

[31] V.M. Abazov et al. (DØ Collaboration), "Search for the Standard Model Higgs Boson in Associated $W H$ Production in $9.7 \mathrm{fb}^{-1}$ of $p \bar{p}$ Collisions with the D0 Detector," Phys. Rev. Lett., vol. 109, p. 121804, Sep 2012.

[32] T. Aaltonen et al. (CDF and DØ Collaborations), "Evidence for a Particle Produced in Association with Weak Bosons and Decaying to a Bottom-Antibottom Quark Pair in Higgs Boson Searches at the Tevatron," Phys. Rev. Lett., vol. 109, p. 071804, Aug 2012.

[33] W. Fisher, "Collie: A Confidence Level Limit Evaluator," DØ Internal Notes, 2008. DØ-NOTE-5595.

[34] G. A. et al. (ATLAS Collaboration), "Observation of a new particle in the search for the Standard Model Higgs boson with the ATLAS detector at the LHC," Physics Letters B, vol. 716, no. 1, pp. 1 - 29, 2012. 
[35] S. Chatrchyan et al. (CMS Collaboration), "Observation of a new boson at a mass of $125 \mathrm{GeV}$ with the CMS experiment at the LHC," Physics Letters B, vol. 716 , no. 1 , pp. $30-61,2012$. 


\section{BIOGRAPHICAL STATEMENT}

Ian J. Howley was born in Baldwin NY, on August 7, 1985 just two months before the first collisions were recorded at the Tevatron. He received his B.S. degree in physics from The College of William and Mary in Virginia in 2008 completing his undergraduate research with Jeff Nelson building the MINER $\nu$ A neutrino detector. He received his M.S. in physics from The University of Texas at Arlington in 2011, while working on development of a fast timing detector for the ATLAS Forward Proton Detector. In addition to his graduate studies Ian worked as a planetarium educator at UTA spreading his passion and excitement for understanding the Universe.

Aside from physics Ian enjoys outdoor pursuits including hiking, rock climbing, soccer and running. 